This document is the accepted manuscript version of the following article:

Vaz, C. A. F., Shin, Y. J., Bibes, M., Rabe, K. M., walker, F. J., \& Ahn, C. H. (2021). Epitaxial ferroelectric interfacial devices. Applied Physics Reviews, 8(4), 041308 (59 pp.). https://doi.org/10.1063/5.0060218

Epitaxial ferroelectric interfacial devices

AIP/123-QED

\title{
Epitaxial ferroelectric interfacial devices
}

C. A. F. Vaz, ${ }^{1, a)}$ Y. J. Shin, ${ }^{2,3}$ M. Bibes, ${ }^{4}$ K. M. Rabe, ${ }^{5}$ F. J. Walker, ${ }^{2,3, b)}$ and C. H. $\mathrm{Ahn}^{3,6, \mathrm{c})}$

1) Swiss Light Source, Paul Scherrer Institut, 5232 Villigen PSI,

Switzerland

${ }^{2)}$ Department of Applied Physics, Department of Electrical Engineering, Yale University, New Haven, Connecticut 06520, USA

3) Center for Research on Interface Structures and Phenomena (CRISP), Yale University, New Haven, CT 06520, USA

4) Unité Mixte de Physique, CNRS, Thales, Université Paris Saclay, 91767 Palaiseau, France

5) Department of Physics and Astronomy, Rutgers University, Piscataway, NJ 08854-8019, USA

${ }^{6)}$ Department of Applied Physics, Department of Physics, and Department of Mechanical Engineering and Materials Science, Yale University, New Haven, CT 06520, USA

(Dated: 11 September 2021) 
Epitaxial ferroelectric interfacial devices

Ferroelectric interfacial devices consist of materials systems whose interfacial electronic properties (such as a 2D electron gas or an interfacial magnetic spin configuration) are modulated by a ferroelectric layer set in its immediate vicinity. While the prototypical example of such a system is the ferroelectric field effect transistor first proposed in the 1950s, only with the recent advances in the controlled growth of epitaxial thin films and heterostructures, and the recent physical understanding down to the atomic scale of screening processes at ferroelectric-semiconducting and -metallic interfaces made possible by first principles calculations, have the conditions been met for a full development of the field. In this review, we discuss the recent advances in ferroelectric interfacial systems, with emphasis on the ferroelectric control of the electronic properties of interfacial devices with well ordered (epitaxial) interfaces. In particular, we consider the cases of ferroelectric interfacial systems aimed at controlling the correlated state, including superconductivity, Mott metallic-insulator transition, magnetism, charge, and orbital order, and charge and spin transport across ferroelectric tunnel junctions. The focus is on the basic physical mechanisms underlying the emergence of interfacial effects, the nature of the ferroelectric control of the electronic state, and the role of extreme electric field gradients at the interface in giving rise to new physical phenomena. Such understanding is key to the development of ferroelectric interfacial systems with characteristics suitable for next generation electronic devices based on controlling the correlated state of matter.

Keywords: Ferroelectrics, heterostructures, devices, field effects, interfaces, multiferroics, thin film epitaxy, ferroelectric tunnel junctions

\footnotetext{
a) Electronic mail: carlos.vaz@psi.ch

b) Electronic mail: fred.walker@yale.edu

c) Electronic mail: charles.ahn@yale.edu
} 
Epitaxial ferroelectric interfacial devices

\section{INTRODUCTION}

The current interest in interface phenomena stems from the desire to discover and explore new physical effects emerging at the immediate atomic region forming the boundary between two different materials, in particular, since the change in crystal symmetry allows new physical phenomena that are distinct from those of the respective bulk phases. As a consequence, modifications in the electronic structure that result from electron transfer or charge confinement effects can lead to the emergence of novel effects and to new functionalities that are intrinsic to the interface. ${ }^{1,2}$ An important class of interfacial systems utilizes a ferroelectric material to modulate the electronic properties of the interfacial system through the ferroelectric polarization. We refer to such device structures as ferroelectric interfacial devices in order to emphasize the fact that such phenomena are characteristic of the interface, with the latter acquiring its own physical, chemical and electronic intrinsic properties. Two different types of device structures can be distinguished: one based on the ferroelectric field effect approach used to induce large and switchable modulations in the charge carrier density at the interface through charge screening effects ${ }^{3-5}$ and the other based on ferroelectric tunnel junctions, where the changes in the electric potential as a function of the direction of the ferroelectric polarization give rise to large changes in the transport characteristics across the ferroelectric used as a tunnel barrier. ${ }^{6,7}$ Important physical requirements for both types of device structures, include sharp interfaces for optimal effects, robust ferroelectric effects at ultrathin thicknesses, and precise control of the channel and ferroelectric layer thickness for ferroelectric field effect and tunnelling devices, respectively. Under such conditions, the ferroelectric control of several interfacial physical phenomena have been achieved, including interfacial magnetism, superconductivity, and orbital order in ferroelectric field effect devices, and of the tunnelling resistance in various ferroelectric tunnel junction architectures, as illustrated in Fig. 1. The control of those physical phenomena involve a laborious identification of the mechanisms linking the ferroelectric order parameter to that of the interfacing material, often a joint experimental and theoretical endeavour. The progress achieved over the last two decades in our understanding of thin film ferroelectricity ${ }^{8-14}$ and of interface phenomena, ${ }^{1,2,15-28}$ from both practical and fundamental perspectives, has been tremendous. The aim of this review is to take stock of the recent developments in the field to gauge the challenges for further progress in view of potential technological applications. We pay par- 
Epitaxial ferroelectric interfacial devices

ticular attention to the aspects associated to ultrathin ferroelectricity, since ferroelectric films are one of the fundamental building blocks of interfacial ferroelectric devices, as well as to the physical mechanisms underlying the emergence and control of the interfacial effects between the ferroelectric and the other material of interest.

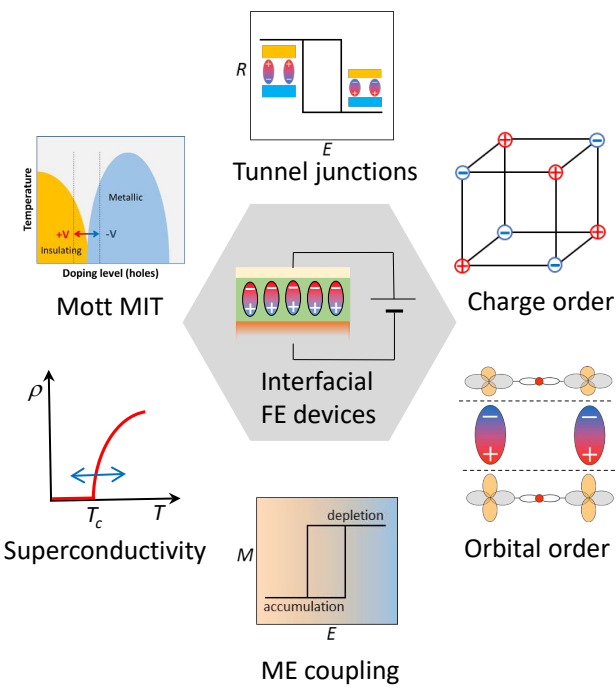

FIG. 1. Schematic diagram showing various types of control of the correlated state in interfacial ferroelectric (FE) devices, including control of superconductivity, Mott metal to insulator (MIT) transition, tunneling conductivity, charge, orbital, and magnetic order.

Ferroelectric materials are characterized by the presence of a spontaneous electric polarization, whose direction can be switched between states that are identical in crystal structure (enantiomorphous) by means of an applied electric field. ${ }^{29,30}$ Ferroelectricity involves space inversion symmetry breaking and the spontaneous ferroelectric polarization can originate from a number of different microscopic mechanisms, including displacement of ions from high symmetry positions, electronic charge asymmetries (as in lone-pair ferroelectricity), charge ordering and/or disproportionation, and geometrical frustration..$^{9,31-40}$ Hence, ferroelectric phenomena tend to be more challenging to investigate than their magnetic counterparts, ${ }^{35,41}$ also due to the strong interaction with the electric fields generated by free electric charges and the strong coupling to lattice distortions, including strain, which often 
Epitaxial ferroelectric interfacial devices

make modelling and interpretation of the experimental results difficult. Theoretically also, only relatively recently a consistent theory of the ferroelectric polarization ${ }^{42-47}$ (expressed in terms of a Berry phase $)^{48}$ and the development of $a b$ initio methods to treat the electric field termination of ferroelectric slabs ${ }^{9,31,34}$ have been made available. From a materials' perspective, ferroelectric systems tend to be multicomponent systems, typically containing three or more elements, with a concomitantly complex defect chemistry that make the preparation of good quality, pure samples difficult, but a task made possible in many instances by the advances in the controlled epitaxial growth of thin ferroelectric films and multilayer heterostructures. The ability to grow high quality thin ferroelectric films is important not only for fundamental studies but also for device applications, where size scaling and a long term retention of the ferroelectric properties at small lateral dimensions and reduced thickness are important requirements, and because the onset of interfacial phenomena between dissimilar materials and the control of those properties by means of external stimuli, such as electric fields, require well defined and abrupt interfaces. In this context, state-of-the-art techniques for ferroelectric thin film growth and optimization of the growth process have resulted in the routine fabrication of epitaxial films of very high structural quality, as we illustrate later for a few prototypical ferroelectric systems (section II B). Also significant has been the development of new experimental techniques dedicated to the study of nanoscale ferroelectricity and interfacial phenomena (section II A); in particular, the development of continuous and operando modes of measurement, where the physical properties of the device are probed while subject to a varying external stimulus, are particularly appealing, since they allow one to follow the changes in physical properties in real time while minimising the influence of other parameters. The combined progress achieved in these theoretical and experimental fronts have led to a strong increase in interest and in the understanding of physical phenomena in ferroelectrics.

Of almost equally importance as the intrinsic properties of ferroelectrics are the conditions under which the ferroelectric system is physically and electrically connected, particularly for thin films, where such boundary conditions have a tremendous impact on the ferroelectric properties. As illustrated in Fig. 2, both three-terminal ferroelectric field effect devices and two-terminal ferroelectric tunnel junctions require a supporting substrate and electrical connections to the ferroelectric layer and to the interface. Since ferroelectricity is generally linked to local structural distortions in the atomic lattice, the constraints imposed by the 
Epitaxial ferroelectric interfacial devices

substrate where the ferroelectric film is grown impacts strongly its ferroelectric properties, including ferroelectric polarisation, critical temperature and piezoelectric response. Electrical boundary conditions are also present in terms of metallic contacts to the ferroelectric film, both to switch the ferroelectric state of the device and to establish the functional interface, for example with a superconductor in a field effect device or with a ferromagnet in a multiferroic ferroelectric tunnel junction. The metallic electrode acts as a charge reservoir that screens the electric polarization at the dielectric interface. In fact, the dielectric/metallic interface has since long received considerable attention since it constitutes the basis of the capacitor structure through which the properties of dielectrics are often investigated. Surprisingly, a strong dependence of the dielectric properties with the electrode material has been observed, in the extreme case leading to a strong reduction of the dielectric properties of the dielectric at reduced thicknesses, built-in electric fields, and fatigue. ${ }^{49-57}$ To a large extent, such phenomena are linked to the physical characteristics of the metal/ferroelectric interface and the key aspects associated with this interface are discussed in section II C.

The prototypical example of a ferroelectric interfacial device is the ferroelectric field effect transistor, first proposed in the $1950 \mathrm{~s},{ }^{58,59}$ before the inventions of the integrated circuit and $\mathrm{SiO}_{2}$ as the transistor gate dielectric. The goal of this invention is to combine the logic function of a CMOS transistor with the switching of the ferroelectric polarization to achieve non-volatile on/off states. Two challenges need to be overcome in such structures: the control of the interface structure in order to avoid spurious screening of the ferroelectric surface polarization that may arise from impurities or charge traps from band gap states and ensuring that the (semi)conducting channel can provide the requisite charge screening of the ferroelectric polarization to stabilize ferroelectricity and the orientation of the ferroelectric polarization. Such challenges prevented the immediate success of the ferroelectric field effect transistor (FeFET), but recent efforts have resulted in approaches to using ferroelectrics in fast and energy efficient memory devices. ${ }^{60}$ The FeFET concept remains very appealing and has been adapted to controlling the properties of other material systems susceptible to charge modulation (Fig. 1a). For example, instead of a semiconductor channel, large changes in the channel conductivity can be achieved by controlling the insulator to metal transition of a Mott insulator, or the superconducting state of a high-critical temperature superconductor. Here, one controls not simply the conductivity through the charge carrier density, but directly the correlated state via its strong dependence on the carrier density. 
Epitaxial ferroelectric interfacial devices
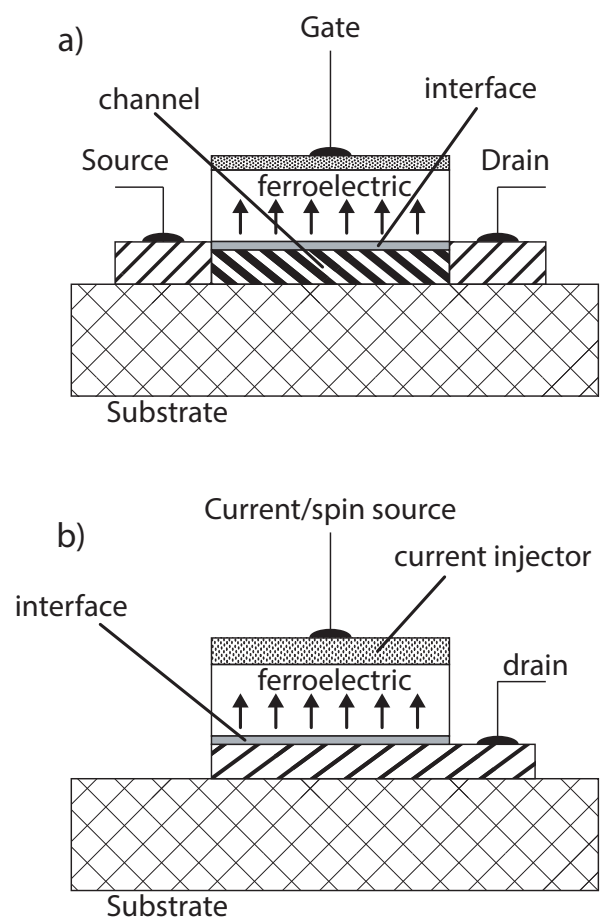

FIG. 2. Schematic of two and three terminal devices based on ferroelectrics and interfacial switching. a) The three terminal device, which is similar to a transistor, consists of source and drain contacts to an active channel. The conductivity of the channel is strongly dependent on interface effects between the channel material and the ferroelectric polarization. b) Tunnel transport in a two-terminal device through the ferroelectric is controlled by the interaction of the ferroelectric polarization and the top and bottom electrode.

This approach to controlling the correlated state of matter through changes in the charge carrier density using the ferroelectric field effect has proved to be a powerful method to observe new physical interfacial phenomena. That the processes are largely confined to the interface is a result of the relatively large charge density of strongly correlated electron systems and the correspondingly short Thomas-Fermi screening lengths of the order of the unit cell. ${ }^{3}$ By taking advantage of the very large surface bound charges that are characteristic of ferroelectrics, of up to one electron per unit cell, one can reach modulations in the interfacial 
Epitaxial ferroelectric interfacial devices

carrier density well beyond what is possible with silicon oxide gates. ${ }^{3}$ The ferroelectric field effect approach has been used to electrostatically control the superconducting state, magnetism, the Mott metal to insulator (MIT) transition, and the orbital state, as we discuss in detail in section III. The challenges for these more exotic field effect devices are increasing the size of the observed effects to above room temperature, as desirable for electronic devices.

While tunneling transport phenomena across dielectrics are now well established, the particular case when the dielectric is also a ferroelectric has been explored only recently, first theoretically, ${ }^{61-64}$ and later experimentally, ${ }^{65-68}$ to reveal surprisingly large changes in conductivity as a function of the ferroelectric polarization direction, in what is termed tunneling electric resistance (TER) in analogy with the magnetic counterpart, tunneling magnetoresistance (TMR) ${ }^{69,70}$ The experimental study of such effects is made possible by the availability of high quality, ultrathin ferroelectric films, as discussed in section II. Several mechanisms are responsible for the TER effect, some related to the bulk of the ferroelectric film, including changes in thickness with the applied electric field, while other contributions are linked to modifications of the electronic potential and changes in bonding at the interface. ${ }^{6} \mathrm{We}$ overview in section IV the current status of the field.

An important motivation for the research work in this field is the prospect of applying the physical effects observed at ferroelectric interfaces to functional devices. ${ }^{71}$ While such systems offer good scaling properties, ${ }^{72}$ novel functionalities, and robust electric field control over electronic properties, the main challenges to realizing ferroelectric devices are the requirement for sustaining the ferroelectric state at ultrathin thicknesses for ferroelectrics of technological interest (which is presently more a practical rather than a fundamental issue) and of achieving sufficiently large interfacial electronic effects at ambient temperature. Also, rather than competing with binary logic operation, the class of multifunctional devices should aim for more complex operations, including devices with selective or selfenforcing memory suitable for simulating neural networks for artificial intelligence, or to control quantum entanglement for quantum computation. ${ }^{73}$ For example, while current artificial neural networks are based on classical computing, it can be anticipated that better scaling, speed, and lower power consumption of the millions of interconnected artificial neurons and synapses can be better achieved with memristor devices (systems showing hysteresis in the resistivity response) based on oxide-based material systems. ${ }^{74}$ In this context, resistive 
Epitaxial ferroelectric interfacial devices

switching associated to reversible, redox processes that give rise to a non-volatile change in resistivity with the applied electric field that develops in many thin films, ${ }^{75}$ including oxides, has been proposed as a potential building block for neural networks. Since they rely on filamentary disruption of the insulating barrier between two conducting electrodes, they can be easily scaled up; however, the control of their onset within a device and the spread in their characteristics remains a challenge. One other device structure capable of the same memristor function is the ferroelectric tunnel junction. ${ }^{76-78}$ Compatibility and integration of such types of devices with current CMOS technology would also be desirable. ${ }^{79}$ A brief overview of the proposals being put forward in this area is discussed in section $\mathrm{V}$.

Given the size of the field, we restrict ourselves to discussing epitaxial systems, where better control over the interface enables a more targeted control of the electronic effects and minimizes extrinsic factors. In particular, our focus is on oxide materials, where the interfacial properties between ferroelectric oxides and complex oxides have received the bulk of the attention and are better understood. Not discussed are heterostructures consisting of two-dimensional (2D) materials interfaced with ferroelectrics; the physical and chemical properties of $2 \mathrm{D}$ materials, such as graphene and the transition metal di- and trichalcogenides, have generated much interest due to the wide range of properties exhibited by these systems, including pseudo-spin in the case of graphene, ${ }^{80,81}$ magnetism, ferroelectricity, and optical properties in the chalcogenides. ${ }^{82-84}$ An increasing body of work has focused on interfacing such materials with ferroelectrics, which has be focus of several recent review articles. ${ }^{85-92}$ The main approach in such device structures has been to modulate the charge carrier density via electrostatic doping and a significant effort has been placed at forming a clean interface between these systems and the ferroelectric to achieve the expected response to the ferroelectric charge modulation. ${ }^{93-95}$ In these systems, epitaxy does not seem critical to device function, which remains at the level of the 2D system itself.

Our goals in this paper are to provide both a timely appraisal of the current developments on ferroelectric interfacial devices and to stimulate further work by pointing, in our view, to promising approaches, as we discuss succinctly in the conclusions and outlook (section VI). Our overview highlights that the large practical and theoretical efforts in fabricating and understanding thin film ferroelectricity have led to a good overall control of the properties of ferroelectric thin films and to the discovery of new ferroelectric systems with enhanced properties that are imminently suited to device applications; in turn, the growth of high 
Epitaxial ferroelectric interfacial devices

quality ferroelectric/complex oxide interfaces have enabled the discovery and study of new interfacial phenomena that have the potential for new functional devices.

\section{THIN FERROELECTRIC FILMS}

\section{A. Ferroelectric characterization techniques}

The experimental investigation of ferroelectric phenomena at the nanoscale requires techniques with sufficient sensitivity and spatial resolution to measure the physical properties of systems at reduced volumes. Measurements of the dielectric and ferroelectric response to electric stimuli, such as permittivity, dielectric loss, breakdown voltage, ferroelectric polarization, piezoelectric response, and ferroelectric switching. Such types of measurements are often carried out in a parallel plate capacitor geometry, with metallic electrodes in direct contact to the ferroelectric system. In this geometry, a time-varying, spatially uniform electric field is applied to the ferroelectric system across the metallic plates and the displacement current is measured as a function of time to yield the dielectric constant for small electric field excitations, or the ferroelectric polarization by integration of the displacement current associated with the ferroelectric polarization switching. Commercial ferroelectric testers can be used to obtain polarization versus electric field hysteresis curves that provide not only measurements of the coercive field, saturation and remanent polarization, but also convey information about the ferroelectric switching process, leakage current, built-in electric fields at the contact interfaces, and fatigue (i.e., loss in switchable polarization upon repeated switching) due to defects. Alternatively, the ferroelectric polarization can be obtained through the pyroelectric effect, by measuring the charge required to compensate the onset of the ferroelectric polarization when cooling the sample down from the paraelectric state. ${ }^{96-98}$ In addition, to probe nanoscale volumes and ferroelectric dynamics, the size of the contacts can be defined to be arbitrarily small, for example, down to the nanoscale by means of advanced e-beam lithography techniques, and on ultrathin ferroelectric films. However, while conceptually simple, such types of electrical measurements are challenging for a number of reasons: in addition to the small magnitude of the signal, tunnelling currents in ultrathin films may overwhelm the ferroelectric displacement currents, and the impact of the metallic electrical contacts needs to be taken into consideration in order not to mask 
Epitaxial ferroelectric interfacial devices

the intrinsic dielectric properties of the ferroelectric system. ${ }^{99,100}$ For example, for PZT, it is found that the $\mathrm{Cu}, \mathrm{Au}, \mathrm{Ag}$, and $\mathrm{Pd}$ are suitable materials that form Schottky contacts, while Ta and Cr can form ohmic contacts, unsuitable for electric characterization. ${ }^{101-104}$ Since the impact of the electric contact/ferroelectric interface is fundamental for ferroelectric field effect devices, we will discuss this aspect in more detail in section II C 2 .

A second type of measurement probes local ferroelectric phenomena at the nano- or atomic scale and employs techniques that have high spatial resolution. One such technique is piezoresponse force microscopy $(\mathrm{PFM}),{ }^{105-108}$ a scanning probe technique that relies on the measurement of the sample piezoelectric response to an alternating electric field applied locally through a conducting tip of an atomic force microscope (in contact mode) and an extended bottom electric contact (typically, a conducting substrate or a conduction layer grown between the substrate and the ferroelectric film). At an elementary level, the amplitude of the response provides a direct measure of the local piezoresponse amplitude, while the phase signal provides direct information about the orientation of the ferroelectric domain (in-phase when the polarization points opposite to the electric field and $180^{\circ}$ for the opposite direction). While a powerful technique for obtaining spatially resolved maps of the ferroelectric domain structure and of the local piezoelectric response, instrumental artifacts, such as local charge injection from the metal tip to the ferroelectric film surface, can make interpretation of PFM results difficult. ${ }^{109}$ Also, quantitative values for the piezoresponse of thin ferroelectric films can be challenging to extract due to the small vertical displacements induced by the electric field and due to the highly non-homogeneous electric field generated by the sharp metallic tip, and multiple strategies have been developed for overcoming such difficulties. ${ }^{110-116}$ With the same proviso, local electric fields can be applied to the sample to directly visualise the ferroelectric switching process. ${ }^{117,118}$ Another technique that has been used to visualize ferroelectric domains is photoemission electron microscopy (PEEM), which measures the intensity of photoemitted electrons excited by a light source, such as ultra-violet light, monochromatic electrons or x-rays. ${ }^{119-122}$ Since the photoemitted electron intensity depends strongly on the surface potential, differences in the latter associated with the ferroelectric polarization of the different domains at the sample surface can be measured to provide direct images of the ferroelectric domains. ${ }^{121,123-126}$ For similar reasons, low energy electron diffraction (LEED) can be used also as a tool to derive the surface potential of ferroelectric surfaces, although without the spatial resolution. ${ }^{56}$ 
Epitaxial ferroelectric interfacial devices

Techniques based on x-rays, and synchrotron x-ray light in particular, are also powerful probes for ferroelectric properties, providing precise measurements of the local atomic structure, including changes in atomic displacements, periodicity of ferroelectric domains, and ferroelectric switching, as a function of electric field, temperature, and pressure via $\mathrm{x}$-ray diffraction and scattering; ${ }^{127-129}$ of the electronic state and band alignment via x-ray photoemission spectroscopy (XPS); ${ }^{130-134}$ and of the electronic band structure using x-ray absorption spectroscopy (XAS). ${ }^{135-141}$ With the advent of free electron laser sources, ${ }^{142}$ capable of producing extremely intense, highly transverse coherent, and ultra-short (few fs long) pulsed x-ray beams, it is now possible to investigate ultrafast dynamical processes in ferroelectric systems, including phonon dynamics and ultrafast switching induced by strong THz light beams. ${ }^{143}$

Finally, we mention here high resolution scanning transmission electron microscopy (HRSTEM), which is a particularly powerful technique for probing in real space the atomic structure of both ferroelectric films and interfaces, and capable also of locally probing some aspects of the electronic structure via electron energy loss spectroscopy (EELS). ${ }^{28,144}$ The direction of the ferroelectric polarization can be determined from an accurate determination of the relative atomic displacement between cation and anion species, such that ferroelectric domains and domain walls can be identified; ${ }^{145-147}$ also from the amplitude of the atomic displacement, estimates of the ferroelectric polarization can be obtained from the relation between effective Born charges and ferroelectric polarization. ${ }^{147-149}$ In many systems, local electrical contacts to the sample enable one to carry out in situ ferroelectric switching studies ${ }^{146}$ including of ferroelectric domain wall dynamics. ${ }^{145,150-152}$ Atomically-resolved EELS spectra can provide additional information on the interface atomic structure (including the presence of atomic interdiffusion),${ }^{153-155}$ valence profile across the interface, ${ }^{147,156-161}$ and atomic layer stacking across the interface. ${ }^{162}$

\section{B. Epitaxial film growth}

A challenging aspect in the study of thin film ferroelectricity relates to the preparation of such systems, typically achieved by means of film growth techniques such as molecular beam epitaxy (MBE), pulsed layer deposition (PLD), and r.f. magnetron sputtering. ${ }^{163-167}$ In such deposition techniques, the film grows on a supporting substrate by condensation 
Epitaxial ferroelectric interfacial devices

of the various elements, or molecules, that compose the material. To promote crystalline order, the growth is carried out at elevated temperature, such that atomic and molecular surface diffusion is high but bulk mass mobility is low. ${ }^{168}$ Often various stable crystalline phases exist for the same material composition, or for a subset of the constituent elements, such that spurious phases may develop during growth which can in some instances strongly impact the materials properties or lead to an inconclusive interpretation of the experimental findings. A related aspect is that, in the majority of cases, the window in parameter space for the growth of the material of interest is relatively narrow, making the growth of high quality epitaxial films a demanding task that requires a good control over stoichiometry and oxidation state; in addition, a low density of defects, low surface and interface roughness, and the avoidance of spurious phases should be attained as well.

For ferroelectric interface devices it is important not only that the ferroelectric interface be sharp but also that the ferroelectric properties are robust, including having a high spontaneous polarization, a high ferroelectric critical temperature, a large breakdown voltage, and that the system can be grown on suitable substrates as high quality thin films. A number of ferroelectric systems fulfill these conditions and have been grown as high quality single crystalline films, a representative subset of which are discussed next and whose bulk properties are listed in Table I.

\section{1. $\mathrm{BaTiO}_{3}$}

Among the ferroelectrics, barium titanate $\left(\mathrm{BaTiO}_{3}\right)$ has played an important historical role in the study of ferroelectricity, since its crystal structure with a small unit cell permitted a more straightforward understanding of the origin of the spontaneous electric polarization in the solid state as compared to the earlier known ferroelectrics, such as the Rochelle salt with a large unit cell, ${ }^{29}$ it is, in addition, a technologically important ferroelectric, due to its robust ferroelectric and optoelastic properties. ${ }^{190-193}$ The high-temperature paraelectric phase of $\mathrm{BaTiO}_{3}$ (between $1733 \mathrm{~K}$ and $393 \mathrm{~K}$ ) crystallizes in the centrosymmetric cubic perovskite structure; at lower temperatures between 393 and $278 \mathrm{~K}$, the active soft-phonon mode associated with the relative displacement of the Ti cation with respect to the oxygen octahedron freezes, leading to the onset of a ferroelectric dipole moment in a tetragonal crystal structure, with the polarization pointing along the $c$ axis. At lower temperatures 
Epitaxial ferroelectric interfacial devices

TABLE I. Bulk properties of selected ferroelectric materials at room temperature; for $\mathrm{HfO}_{2}$, only thin films have been stabilized. Values from Ref. 169 except where mentioned otherwise. Structure: T: tetragonal, R: rombohedral, O: orthorhombic. $T_{\mathrm{c}}$ : ferroelectric critical temperature, $E_{c}$ : coercive field, $E_{\mathrm{g}}$ : band gap, $\chi$ : electron affinity, $\epsilon_{r}$ : relative dielectric constant $\epsilon_{33} / \epsilon_{0}$, and $\epsilon_{\infty}$ : relative electronic dielectric constant (taken as the square of the ordinary refractive index $n_{\mathrm{o}}$ at $633 \mathrm{~nm}$ ). ${ }^{170}$

\begin{tabular}{|c|c|c|c|c|c|c|c|c|c|}
\hline Material & Str. & $\begin{array}{l}\text { Latt. Param. } \\
(\AA)\end{array}$ & $\begin{array}{r}T_{\mathrm{c}} \\
(\mathrm{K}) \\
\end{array}$ & $\begin{array}{r}P_{\mathrm{S}} \\
\left(\mu \mathrm{C} / \mathrm{cm}^{2}\right) \\
\end{array}$ & $\begin{array}{r}E_{c} \\
(\mathrm{kV} / \mathrm{cm})\end{array}$ & $\begin{array}{c}E_{\mathrm{g}} \\
(\mathrm{eV}) \\
\end{array}$ & $\begin{array}{c}\chi \\
(\mathrm{eV}) \\
\end{array}$ & $\epsilon_{r}$ & $\epsilon_{\infty}$ \\
\hline $\mathrm{BaTiO}_{3}$ & $\mathrm{~T}$ & $\left\{\begin{array}{l}a=3.9920 \\
c=4.0361\end{array}\right.$ & 393 & 25 & 1.2 & $3.2^{\mathrm{a}}$ & $3.8^{1}$ & 130 & $5.79^{\mathrm{h}}$ \\
\hline $\mathrm{PbTiO}_{3}$ & $\mathrm{~T}$ & $\left\{\begin{array}{l}a=3.9072 \\
c=4.1187\end{array}\right.$ & 763 & 75 & 6.75 & $3.2^{\mathrm{a}}$ & $2.8^{\mathrm{m}}$ & 200 & $7.16^{\mathrm{h}}$ \\
\hline $\mathrm{Pb}\left(\mathrm{Zr}_{0.2} \mathrm{Ti}_{0.8}\right) \mathrm{O}_{3}$ & $\mathrm{~T}$ & $\left\{\begin{array}{l}a=3.933 \\
c=4.134\end{array}\right.$ & $730^{\mathrm{d}}$ & $70^{\mathrm{d}}$ & $100^{\mathrm{d}}$ & $3.4^{\mathrm{a}}$ & $3.5^{\mathrm{g}}$ & $90^{\mathrm{n}}$ & $6.30^{\mathrm{j}}$ \\
\hline $\mathrm{BiFeO}_{3}$ & $\mathrm{R}$ & $\left\{\begin{array}{l}a=5.616 \\
\alpha=59.35^{\circ}\end{array}\right.$ & 1123 & $100^{\mathrm{f}}$ & $10^{\mathrm{f}}$ & $2.8^{\mathrm{a}}$ & $4.0^{\circ}$ & 65 & $8.82^{\mathrm{i}}$ \\
\hline $\mathrm{HfO}_{2}$ & $\mathrm{O}$ & $\begin{array}{l}a=5.07^{\mathrm{e}} \\
b=4.88^{\mathrm{e}} \\
c=4.89^{\mathrm{e}}\end{array}$ & $723^{\mathrm{c}}$ & $15-45^{\mathrm{c}}$ & $1000^{\mathrm{c}}$ & $5.5^{\mathrm{c}}$ & $1.7^{\mathrm{P}}$ & $23^{\mathrm{c}}$ & $3.98^{\mathrm{k}}$ \\
\hline
\end{tabular}

${ }^{\text {a }}$ Ref. 171

b Ref. 172

${ }^{c}$ Ref. 173

${ }^{\mathrm{d}}$ Ref. 174

${ }^{\text {e }}$ First principles calculations, Ref. 175

${ }^{\mathrm{f}}$ Ref. 176

g Ref. 177

${ }^{\mathrm{h}}$ Ref. 178

${ }^{\mathrm{i}}$ Ref. 179

j Ref. 180

${ }^{k}$ Ref. 181 and 182

${ }^{1}$ Ref. 183

${ }^{m}$ Ref. 184

${ }^{n}$ Ref. 185 and 186

${ }^{\circ}$ Ref. 187

p Ref. 188. For $\mathrm{HfO}_{2} / \mathrm{Si}$, using the electron affinity of $\mathrm{Si}$ as $4.05 \mathrm{eV} .^{189}$ 
Epitaxial ferroelectric interfacial devices

(between 278 and $183 \mathrm{~K}$ ) a further distortion in the crystal structure to orthorhombic takes place, along with a change in the direction of the ferroelectric polarization to the pseudocubic $[110]_{\mathrm{ps}}$ direction; below $183 \mathrm{~K}$, the structure changes further to rhombohedral with the polarization pointing along the pseudocubic $[111]_{\mathrm{ps}}$ direction. ${ }^{169,194}$

Although high quality $\mathrm{BaTiO}_{3}$ single crystals are commercially available, its high cost, its sensitivity to variations in temperature due to the tetrahedral to orthorhombic phase transition at $278 \mathrm{~K}$, and the long switching times and high voltages required to excite bulk crystals has driven the need for the growth of high quality epitaxial films on standard substrate materials for use in device applications. Epitaxial thin films of $\mathrm{BaTiO}_{3}$ have been grown by MBE, PLD and sputtering on various substrates, including metal oxides such as $\mathrm{SrTiO}_{3}(001),{ }^{49,195-214} \mathrm{MgO}(001),{ }^{196,215-219} \mathrm{GdScO}_{3}(110),{ }^{218,220} \mathrm{DyScO}_{3}(110),{ }^{218}$ $\mathrm{LaAlO}_{3}(100),{ }^{217} \mathrm{MgAl}_{2} \mathrm{O}_{4}(001),{ }^{221}$ as well as on semiconductors, including $\mathrm{Si}(001),{ }^{222-229}$ and $\mathrm{Ge}(001) .{ }^{230-232}$ In most instances, an intermediate buffer layer is used as an electrical contact or for the release of misfit strain. $\mathrm{BaTiO}_{3}$ films are typically grown at elevated temperatures, well above the critical temperature, such that they are brought down from the paraelectric state to the ferroelectric state at room temperature upon cooling. The most relevant film orientation for $\mathrm{BaTiO}_{3}$ in ferroelectric interfacial devices is the [001], i.e., with the polar axis pointing along the out of plane direction. It is worth pointing out that for the ferroelectric perovskites, the value of the $c / a$ ratio tends to relate to the amplitude of the electric dipole and therefore to the amplitude of the ferroelectric polarization (section II C). ${ }^{198,233-240}$ Hence, a substrate of high interest is $\mathrm{SrTiO}_{3}$, where a lattice mismatch of $-2.18 \%$ is expected to lead to a strong compressive strain and to a large $c / a$ ratio. ${ }^{236}$ On $\mathrm{SrTiO}_{3}(001), \mathrm{BaTiO}_{3}$ is found to grow layer by layer in steps of one unit cell (u.c.) up to about 12 u.c., above which the film growth proceeds in a three-dimensional island (Stranski-Krastanov) growth mode; ${ }^{195,200,201,205,208,241}$ at substrate temperatures of the order of $680^{\circ} \mathrm{C}$, typical for oxide film growth, the adatom surface mobility is high, as deduced from the recovery of the RHEED intensity after a break in the deposition process of about 20 s. ${ }^{195,202,205}$ However, such adatom surface mobility seems to be detrimental to the growth of smooth and fully strained films: the latter can be achieved at lower deposition temperatures and higher deposition rates, while under opposite conditions early strain relaxation and 3D growth are observed. ${ }^{204,242,243}$ For the $\mathrm{BaTiO}_{3} / \mathrm{SrTiO}_{3}(001)$ system, the critical thickness for coherent growth (coherent films have the same in-plane lattice parameter and a symmetry 
Epitaxial ferroelectric interfacial devices

compatible with the substrate) has been found to range from 2-10 nm, depending on the growth conditions, above which the in-plane lattice constant starts relaxing towards the bulk value through the onset of misfit dislocations. ${ }^{201,205,207,214,243,244}$ Although Nb-doped conducting $\mathrm{SrTiO}_{3}$ can be used as a substrate for the $\mathrm{BaTiO}_{3}$ film growth to also form a bottom contact, an alternative solution consists of using a conducting buffer layer, such as $\mathrm{LaNiO}_{3},{ }^{206} \mathrm{SrRuO}_{3},{ }^{209,211,245} \mathrm{La}_{1-x} \mathrm{Sr}_{x} \mathrm{MnO}_{3},{ }^{211} \mathrm{YBa}_{2} \mathrm{Cu}_{3} \mathrm{O}_{7-x},{ }^{196,246}$ or $\mathrm{Sr}_{2} \mathrm{RuO}_{4},{ }^{212}$ in the latter case leading to atomically abrupt interfaces, as shown in Fig. 3(a).

Other oxides used for the epitaxial growth of $\mathrm{BaTiO}_{3}$ include $\mathrm{MgO}$ (rocksalt with a lattice constant $a=4.2117 \AA$ ), ${ }^{247}$ and $\mathrm{LaAlO}_{3}$ (rhombohedral below $800 \mathrm{~K}$ with $a=5.365 \AA$, $c=13.11 \AA$, with a pseudo-cubic lattice constant $\left.a_{\mathrm{ps}}=3.79 \AA\right),{ }^{248}$ both of which have a large lattice mismatch to $\mathrm{BaTiO}_{3}$, respectively of $+5.0 \%$, and $-5.5 \%$. Likely as a consequence, the $\mathrm{BaTiO}_{3}$ film quality tends to be poorer in comparison to films grown on $\mathrm{SrTiO}_{3}$, even when buffer layers are incorporated that act to reduce the lattice misfit. ${ }^{196,216,217,219}$ Metal oxide substrates with smaller lattice misfits include $\mathrm{MgAl}_{2} \mathrm{O}_{4}$ (cubic spinel with $a=8.0831$ $\AA$ ), ${ }^{248}, \mathrm{DyScO}_{3}$ (orthorhombic, $a=5.43 \AA, b=5.71 \AA, c=7.89 \AA ; a_{\mathrm{ps}}=3.94 \AA$ ),${ }^{249}$ and $\mathrm{GdScO}_{3}$ (orthorhombic, $a=5.487 \AA, b=5.756 \AA, c=7.925 \AA$; $a_{\mathrm{ps}}=3.98 \AA$ ). ${ }^{250}$ For $\mathrm{BaTiO}_{3}$ films grown on $\mathrm{MgAl}_{2} \mathrm{O}_{4}(001)$, where the lattice misfit is about $0.79 \%$, it is found that relatively high growth temperatures $\left(900-1050^{\circ} \mathrm{C}\right)$ are required for full epitaxy, but with a defective interface as determined by TEM, possibly as a consequence of surface reconstruction of $\mathrm{MgAl}_{2} \mathrm{O}_{4}$, surface terraces exposing different surface terminations, and the large thermal misfit. ${ }^{221} \mathrm{GdScO}_{3}(110)$ and $\mathrm{DyScO}_{3}(110)$ have small lattice misfits to $\mathrm{BaTiO}_{3}$, respectively of $-1.0 \%$ and $-1.7 \%$ and provide excellent templates for the epitaxial growth of fully strained $\mathrm{BaTiO}_{3}(001)$ films with sharp interfaces, as illustrated by the example shown in Fig. 3(b). ${ }^{218,220}$

The growth of complex oxides on semiconducting substrates, particularly Si, has been intensively investigated, with the aim of integrating complex oxides in semiconductor platforms to realize multifunctional electronic and electro-optic devices ${ }^{167,251-260}$ and for using high- $\kappa$ dielectric oxides as alternatives to $\mathrm{SiO}$ for gate dielectrics in MOSFET transistors. ${ }^{170,261-263}$ The challenge is to achieve epitaxial growth and a sharp interface without the formation of an interfacial oxide amorphous layer that may be formed by exposure of the clean semiconducting surface to the oxygen species required for the metal oxide growth or due to reaction with the oxide layer, that could degrade device performance. In the case of $\mathrm{Si}$, sharp and 
Epitaxial ferroelectric interfacial devices

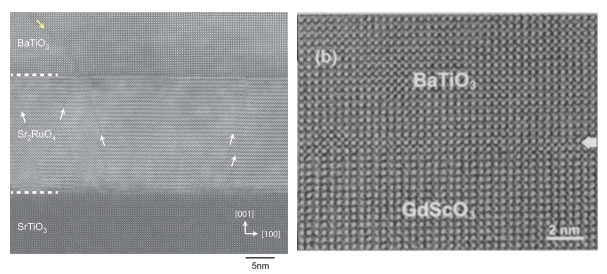

FIG. 3. (a) Cross-sectional high angle annular dark field STEM image of a $\mathrm{BaTiO}_{3}$ film grown on $\mathrm{Sr}_{2} \mathrm{RuO}_{4}(001)$ electrode. (b) Cross-sectional HR-TEM of $1 \mu \mathrm{m}$ thick $\mathrm{BaTiO}_{3} / \mathrm{GdScO}_{3}(110)$ near the interface (indicated by the arrow). (a) Reprinted with permission from ACS Appl. Mater. Interfaces 9, 21314 (2017). Copyright 2017 American Chemical Society. (b) Reprinted from Appl. Phys. Lett. 82, 3460 (2003), with the permission of AIP Publishing.

abrupt interfaces, without the formation of an amorphous interfacial silicon oxide layer, were obtained for $\mathrm{BaO}, \mathrm{SrO}$, and $\mathrm{SrTiO}_{3} \cdot{ }^{252,264-268}$ In these instances, the process relies on the passivation of the Si surface with a submonolayer-thick alkaline metal layer that protects it from oxidation while enabling the start of the oxide film growth. A recent review of the growth of $\mathrm{BaTiO}_{3}$ on $\mathrm{Si}, \mathrm{Ge}$, and GaAs substrates by MBE has been provided by Mazet et $a l .{ }^{254}$ In most instances reported in the literature, the growth of epitaxial $\mathrm{BaTiO}_{3}$ on $\mathrm{Si}(001)$ proceeds through the use of a mediating $\mathrm{SrTiO}_{3}$ buffer layer, which has the added benefit of reducing the large misfit between $\mathrm{Si}\left(a=5.431 \AA\right.$ ) and $\mathrm{BaTiO}_{3}$ (of about $-3.75 \%$ ), which otherwise would favour the growth of $a$-oriented films. It is found that the lattice orientation ( $a$ or $c$ ) depends strongly on the $\mathrm{SrTiO}_{3}$ buffer layer thickness, surface quality, and oxygen partial pressure during growth, making the ferroelectric state difficult to control. ${ }^{254}$ Ge $(a=5.658 \AA)$ has a smaller lattice misfit to $\mathrm{BaTiO}_{3}$, of about $+0.27 \%$ at room temperature, on which high quality $\mathrm{BaTiO}_{3}$ films can be grown directly. ${ }^{265}$ The in-plane tensile strain leads to a preferred $a$-orientation; however, this can be circumvented by using buffer layers that induce a compressive in-plane strain, such as $\mathrm{Ba}_{1-x} \mathrm{Sr}_{x} \mathrm{TiO}_{3},{ }^{230}$ or $\mathrm{SrTiO}_{3} .{ }^{269}$ One example of the $\mathrm{BaTiO}_{3}$ film quality that is possible to achieve on $\mathrm{Ge}(001)$ is shown in Fig. 4 for a 2.5 u.c. film grown by reactive molecular beam epitaxy by first depositing a 0.5 monolayers of $\mathrm{BaO}$ to passivate the Ge surface against oxidation, followed by the deposition of two monolayers each of $\mathrm{BaO}$ and $\mathrm{TiO}_{2}$ to form an amorphous $\mathrm{BaTiO}_{3}$ layer that is subsequently crystallized upon annealing. ${ }^{270}$ The TEM image in Fig. 4(a) shows full crystallization of the 
Epitaxial ferroelectric interfacial devices

$\mathrm{BaTiO}_{3}$ layer, in perfect registry to the Ge lattice; the analysis of crystal truncation rod (CTR) x-ray diffraction measurements ${ }^{271}$ together with the results of $a b$ initio calculations could show that, for this thin $\mathrm{BaTiO}_{3}$ film, rumpling of the $\mathrm{BaO}$ interfacial layer occurs in response to Ge dimerization [Fig. 4(b,c)] and leads to distortions in the $\mathrm{BaTiO}_{3}$ unit cell away from the tetragonal distortion akin to the orthorhombic phase of $\mathrm{BaTiO}_{3}$; a 5.5 u.c. $\mathrm{BaTiO}_{3}$ film grown by additional deposition of three monolyers of $\mathrm{BaO}$ and $\mathrm{TiO}_{2}$ at elevated temperature show the tetragonal structure expected for bulk $\mathrm{BaTiO}_{3}$ at room temperature, however. ${ }^{270}$ Another study has demonstrated epitaxial growth of [001]-oriented $\mathrm{BaTiO}_{3}$ on $\mathrm{Ge}(001)$ through the deposition of a $2 \mathrm{~nm}$ amorphous $\mathrm{BaTiO}_{3}$ film that was subsequently crystallized upon annealing and that could be used as a seed for further $\mathrm{BaTiO}_{3}$ growth. ${ }^{232}$ Although GaAs $(a=5.65325 \AA)$ also has a small lattice misfit to $\mathrm{BaTiO}_{3}$ of about $+0.19 \%$, the lack of structural and chemical stability of the GaAs surface to the high temperatures required for $\mathrm{BaTiO}_{3}$ growth and interdiffusion precludes direct growth of $\mathrm{BaTiO}_{3}$ on $\mathrm{GaAs}$, making the use of a buffer layer, such as $\mathrm{MgO}$ or $\mathrm{SrTiO}_{3}$, necessary for high quality single crystalline films. ${ }^{254,272}$ In this sense, the problem is reduced to one of growing the buffer oxide layer on GaAs. ${ }^{254,273}$

(a)

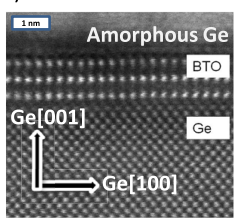

(b)

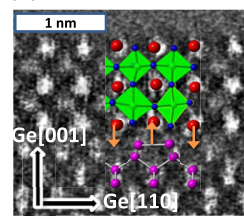

(c)

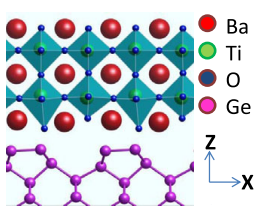

FIG. 4. (a) High angle annular dark field TEM image of 2.5 u.c. $\mathrm{BaTiO}_{3} / \mathrm{Ge}(001)$. (b) Superimposed on the TEM image taken along the Ge [110] zone axis is a model structure of the system determined from surface x-ray diffraction measurements. The red arrows indicate the rumpling of the interfacial Ba along the [001] axis. (c) DFT-predicted ground state structure of the 2.5 u.c. BTO/Ge system. Reprinted with permission from Phys. Rev. Lett. 116, 106101 (2016). Copyright 2016 by the American Physical Society. 
Epitaxial ferroelectric interfacial devices

\section{2. $\mathrm{PbTiO}_{3}, \mathrm{PbTiO}_{3}$-based materials}

Other important perovskite ferroelectrics are $\mathrm{PbTiO}_{3}$ and $\mathrm{PbTiO}_{3}$-based materials. Since the first reports of the crystal structure and ferroelectricity in the $1940 \mathrm{~s},{ }^{274-277} \mathrm{PbTiO}_{3}$ has attracted significant interest due to its excellent ferroelectric properties, ${ }^{60,278,279}$ including a high Curie temperature and a large ferroelectric polarization. At ambient conditions, $\mathrm{PbTiO}_{3}$ presents a tetragonal structure with a large $c / a \approx 1.063 .{ }^{280}$ The Ti cations are off-centered from the oxygen octahedra, inducing a ferroelectric polarization in the system. The ferroelectric phase is stable up to $763 \mathrm{~K}$, then undergoes a transition to the paraelectric phase accompanied by a structural transition from tetragonal to cubic symmetry. ${ }^{281}$ The spontaneous polarization of $\mathrm{PbTiO}_{3}$ crystals is around $75 \mu \mathrm{C} / \mathrm{cm}^{2}$, which is much larger than that of $\mathrm{BaTiO}_{3}$ (see Table I). In addition to the contribution from hybridization of Ti $3 \mathrm{~d}$ and $\mathrm{O} 2 \mathrm{p}$ orbitals, the 6 s lone electron pair of the $\mathrm{Pb}^{2+}$ cations and hybridization between the $\mathrm{Pb} 6 \mathrm{~s}$ and $\mathrm{O} 2 \mathrm{p}$ orbitals provide an additional driving force for the structural distortion characteristic of $\mathrm{PbTiO}_{3} \cdot{ }^{34,282-284}$ The ferroelectric distortion for $\mathrm{PbTiO}_{3}$ differs from that of $\mathrm{BaTiO}_{3}$ in that the oxygen octahedra moves in the same direction, but with larger magnitude, than the Ti cation.

Given its outstanding ferroelectric characteristics, the epitaxial growth of $\mathrm{PbTiO}_{3}$ thin films has been extensively studied for decades. Various deposition methods, such as MBE, PLD, chemical vapor deposition (CVD), and sputtering, have been exploited to achieve highquality epitaxial thin films. ${ }^{285-289}$ The majority of these studies employ [001]-oriented $\mathrm{SrTiO}_{3}$ substrates. On $\mathrm{SrTiO}_{3}(001), \mathrm{PbTiO}_{3}$ adatoms exhibit ideal 2D layer-by-layer growth ${ }^{290}$ or even step-flow growth ${ }^{291}$ to form atomically flat surfaces and interfaces. Coherent growth without undesired a-domain formation has been observed up to a film thickness of 340 $\mathrm{nm},{ }^{292}$ which is far thicker than the coherent critical thickness expected from the MatthewsBlakeslee model. The minimum thickness for the onset of ferroelectricity is reported to be extremely small (three-unit cells) on $\mathrm{SrTiO}_{3} .{ }^{127}$ These features make $\mathrm{PbTiO}_{3} / \mathrm{SrTiO}_{3}(001)$ an ideal platform for ferroelectric interface devices. The successful synthesis of epitaxial $\mathrm{PbTiO}_{3}$ films has been also reported on other substrates, such as $\mathrm{LaAlO}_{3},{ }^{293} \mathrm{MgO},{ }^{294} \mathrm{Pt},{ }^{295}$ and $\mathrm{Si}^{286,287}$

Lead zirconate titanate, $\mathrm{PbZr}_{x} \mathrm{Ti}_{1-x} \mathrm{O}_{3}(\mathrm{PZT})$, is the most widely used ferroelectric and piezoelectric material due to its large ferroelectric spontaneous polarization and piezoelec- 
Epitaxial ferroelectric interfacial devices

tric coefficient. PZT can be seen as a solid solution of $\mathrm{PbTiO}_{3}$ and $\mathrm{PbZrO}_{3}$, an antiferromagnetic rhombohedrally distorted perovskite material. The phase diagram of PZT is characterized by an equilibrium tetragonal phase at large $\mathrm{PbTiO}_{3}$ content $(x \lesssim 0.5)$ and several rhombohedral structures for the $\mathrm{PbZrO}_{3}$ rich phases $(x \gtrsim 0.5) .{ }^{296,297}$ At $x \approx 0.52$, PZT is characterized by the coexistence of tetragonal, rhombohedral, and a more recently identified intermediate monoclinic phase, ${ }^{298-303}$ defining the so-called morphotropic phase boundary; such phase coexistence is responsible for the large piezoelectric response of PZT at this composition. ${ }^{165,297,304-306}$ In the context of interfacial ferroelectric devices, the tetragonal phase of PZT is the most interesting on account of the higher Curie temperature and a larger ferroelectric polarization along the tetragonal axis. ${ }^{174,235,301,307-310}$ The epitaxial growth of thin PZT films has been demonstrated on several substrates using various deposition techniques, as discussed in detail in Ref. 165 and we consider here more recent examples reported in the literature. Due to the volatile $\mathrm{Pb}$ element, PZT films are grown less often by $\mathrm{MBE}^{311}$ and instead by rf sputtering or PLD, typically on Nb-doped $\mathrm{SrTiO}_{3}$ substrates or on undoped $\mathrm{SrTiO}_{3}$ with a conducting oxide buffer layer, which provide a good template for the growth of tetragonal PZT $(x<0.5) .{ }^{312}$ For $x=0.2$, the lattice mismatch to $\mathrm{SrTiO}_{3}$ is $-1.2 \%$ and the films can grow coherently up to a thickness of about $15 \mathrm{~nm}^{313,314}$ The ferroelectric properties of PZT films have been shown to depend sensitively on the condition of the substrate surface: high quality $\mathrm{PbZr}_{0.2} \mathrm{Ti}_{0.8} \mathrm{O}_{3}$ films grown by PLD on single terminated, atomically flat $\mathrm{SrTiO}_{3}(001)$ substrates using a $20 \mathrm{~nm}$ thick $\mathrm{SrRuO}_{3}$ buffer layer were found to grow layer-by-layer, as deduced from atomic force microscopy, and possess a high saturation ferroelectric polarization value of $105 \mu \mathrm{C} / \mathrm{cm}^{2}$ (attributed to a slightly higher value of the $c / a$ ratio, 1.06, as compared to the bulk value of 1.051). ${ }^{186} \mathrm{TEM}$ data for a $90 \mathrm{~nm}$ thick film, reproduced in Fig. 5(a-d), show abrupt, defect-free interfaces; in comparison, a PZT film grown on a less perfect $\mathrm{SrTiO}_{3}$ surface shows the presence of defects, as shown in Fig. 5(e), and is also characterized by a smaller ferroelectric polarization value of $65 \mu \mathrm{C} / \mathrm{cm}^{2} .{ }^{186} \mathrm{PZT}$ films deposited on $\mathrm{La}_{0.8} \mathrm{Sr}_{0.2} \mathrm{MnO}_{3} / \mathrm{SrTiO}_{3}(001)$ exhibit larger ferroelectric polarizations, of up to $85 \mu \mathrm{C} / \mathrm{cm}^{2}$, when the $\mathrm{La}_{0.8} \mathrm{Sr}_{0.2} \mathrm{MnO}_{3}$ film is grown by $\mathrm{MBE}$ on single terminated $\mathrm{TiO}_{2}$ $\mathrm{SrTiO}_{3}$ substrates ${ }^{315,316}$ as compared to PZT/LSMO films deposited by off axis rf-sputtering on mixed terminated $\mathrm{SrTiO}_{3}$ substrates, where the ferroelectric polarization is typically of the order of $45 \mu \mathrm{C} / \mathrm{cm}^{2}$. 317

The $\mathrm{PbTiO}_{3}$-based relaxor-ferroelectrics are also materials of high interest. Relaxor- 
Epitaxial ferroelectric interfacial devices

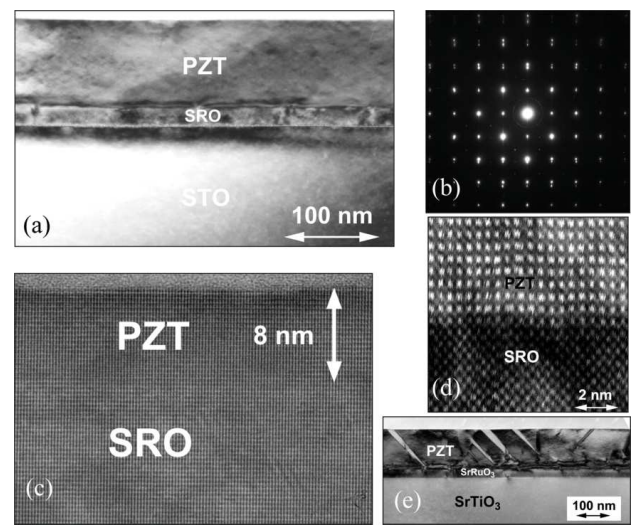

FIG. 5. TEM results for $\mathrm{PbZr}_{0.2} \mathrm{Ti}_{0.8} \mathrm{O}_{3} / \mathrm{SrRuO}_{3} / \mathrm{SrTiO}_{3}$ heterostructures, showing (a) a crosssectional image of a defect-free $90 \mathrm{~nm}$ thick PZT film and (b) corresponding electron diffraction pattern. (c) High-resolution TEM (HRTEM) image of an $8 \mathrm{~nm}$ thick PZT film and (d) Z-contrast STEM image showing the sharp and dislocation-free interface between the $\mathrm{PZT}$ and $\mathrm{SrRuO}_{3}$ layers. (e) Cross-sectional TEM image of a defective $120 \mathrm{~nm}$ thick PZT film. Reproduced with permission from Adv. Mater. 18, 1657 (2006). Copyright 2006 by WILEY-VCH Verlag GmbH \& Co. KGaA, Weinheim.

$\mathrm{PbTiO}_{3}$ ferroelectrics, including $\mathrm{Pb}\left(\mathrm{Mg}_{1 / 3} \mathrm{Nb}_{2 / 3}\right) \mathrm{O}_{3}-\mathrm{PbTiO}_{3}$ (PMN-PT), $\mathrm{Pb}\left(\mathrm{Zn}_{1 / 3} \mathrm{Nb}_{2 / 3}\right) \mathrm{O}_{3^{-}}$ $\mathrm{PbTiO}_{3}$ (PZNT), and $\mathrm{Pb}\left(\mathrm{Zn}_{1 / 3} \mathrm{Nb}_{2 / 3}\right) \mathrm{O}_{3}-\mathrm{PbTiO}_{3}$ (PZN-PT) have been extensively studied due to their ultra-high piezoelectric response, with piezoelectric coefficient $d_{33}>1500$ $\mathrm{pC} / \mathrm{N}$ and electromechanical coupling $k_{33} \approx 90 \%,{ }^{318,319}$ which exceed those of high-quality PZT films. Although the origin of the high piezoelectric response remains to be clarified, it makes relaxor- $\mathrm{PbTiO}_{3}$ ferroelectrics ideal materials for electroacoustic transducers and actuators. ${ }^{320}$ While the majority of the reports on relaxor- $\mathrm{PbTiO}_{3}$ ferroelectrics still focus on single crystals or thick films, thin films of relaxor- $\mathrm{PbTiO}_{3}$ ferroelectrics have also been extensively studied. Magnetron sputtering and PLD have been used to synthesize epitaxial relaxor- $\mathrm{PbTiO}_{3}$ thin films on various substrates, such as $\mathrm{SrTiO}_{3},{ }^{321,322} \mathrm{LaAlO}_{3},{ }^{293,323,324}$ $\mathrm{MgO}^{325}(\mathrm{La}, \mathrm{Sr})(\mathrm{Al}, \mathrm{Ta}) \mathrm{O}_{3}$ (LSAT), ${ }^{326}$ and $\mathrm{Si}^{327,328}$ The ultra-high piezoelectric effect of the relaxor- $\mathrm{PbTiO}_{3}$ ferroelectric crystal also opens a new route to study strain engineering. Most studies on strain effects are based on the synthesis of films with different thicknesses 
Epitaxial ferroelectric interfacial devices

(with different strain relaxation states) or on films grown on different substrates to induce a misfit strain. These methods, however, have the drawback that spurious effects related to slightly different growth conditions between different samples cannot be excluded. On the other hand, using relaxor-PTO ferroelectric crystals as a substrate enables electric control of strain in a single sample, an approach that has been used to study the effect of strain on ferroelectricity ${ }^{329,330}$ and ferromagnetism, ${ }^{331-334}$ for instance.

\section{3. $\mathrm{BiFeO}_{3}$}

$\mathrm{BiFeO}_{3}$ is a well-known multiferroic material characterized by high ferroelectric and antiferromagnetic (Néel) ordering temperatures of $T_{\mathrm{c}}^{\mathrm{e}}=1123 \mathrm{~K}$ and $T_{\mathrm{N}}^{\mathrm{m}}=643 \mathrm{~K}$, respectively, ${ }^{169}$ making it one of the relatively few room-temperature multiferroic compounds. At ambient conditions, $\mathrm{BiFeO}_{3}$ has a rhombohedral structure with ferroelectric easy-axis along $\langle 111\rangle_{\mathrm{pc}}$ and a high spontaneous polarization in excess of $\sim 100 \mu \mathrm{C} / \mathrm{cm}^{2}{ }^{176,335-338}$ Unlike typical displacive ferroelectrics, such as $\mathrm{BaTiO}_{3}$, where off-centering of the Ti cations within the $\mathrm{O}$ cage is at the origin of the ferroelectric polarization, the main contribution to the ferroelectric polarization of $\mathrm{BiFeO}_{3}$ arises from lone pair $\mathrm{Bi} 6 \mathrm{~s}$ orbitals. ${ }^{339,340}$ This allows $\mathrm{BiFeO}_{3}$ to have partially filled $d$ shells on the $\mathrm{Fe}^{3+}$ site (and a spin moment) while ferroelectricity arises largely independently from the $\mathrm{Bi}^{3+}$ cations. Despite the independent origin of ferroelectric and magnetic order, the presence of Dzyaloshinskii-Moriya interactions results in a sizable magnetoelectric coupling between the two order parameters, ${ }^{341,342}$ which has been explored in novel artificial multiferroic heterostrutures for electric field control of magnetism. ${ }^{343-349}$ The magnetic structure of $\mathrm{BiFeO}_{3}$ is locally $\mathrm{G}$-type antiferromagnetic, but the presence of a significant Dzyaloshinskii-Moriya interaction (DMI) associated with the polar displacement ${ }^{350,351}$ induces both a spin canting between adjacent spins (resulting in a small local net spin moment) and an incommensurate cycloidal winding of the spins within a cycloidal plane defined by the direction of the ferroelectric direction and the direction of propagation of the cycloid, with a periodicity of about $64 \mathrm{~nm},{ }^{352-354}$ leading to an effective zero magnetic moment. For a ferroelectric polarisation state pointing along $[111]_{\mathrm{pc}}$, the possible propagation directions for the spin cycloid are $[\overline{1} 10]_{\mathrm{pc}},[01 \overline{1}]_{\mathrm{pc}}$, and $[10 \overline{1}]_{\mathrm{pc}}$. An order of magnitude smaller second DMI interaction, resulting from the alternate rotation of the $\mathrm{FeO}_{6}$ octahedra along the $[111]_{\mathrm{pc}}$ direction, ${ }^{351,355}$ causes the cycloid to be canted about $1^{\circ}$ from the cycloidal plane, inducing 
Epitaxial ferroelectric interfacial devices

a spin density wave. ${ }^{354,356-358}$ Under the effect of epitaxial strain, the spin cycloid can be suppressed or modulated in thin films. ${ }^{354,359-363}$

The first report of the growth of multiferroic epitaxial $\mathrm{BiFeO}_{3}$ thin films ${ }^{364}$ generated a strong interest in this system, well documented in the literature. ${ }^{365-370}$ Here, we mention only the case of $\mathrm{BiFeO}_{3}$ films grown on $\mathrm{LaAlO}_{3}$ crystals which, due to the large compressive epitaxial strain $(\approx 4.5 \%)$, have been found to stabilize in a pseudo-tetragonal phase with a very large $c / a$ ratio of $1.25-1.3,,^{239,370,371}$ characterized by an enhanced spontaneous polarization of over $150 \mu \mathrm{C} / \mathrm{cm}^{2} .{ }^{372}$ The large polarization value of pseudo-tetragonal $\mathrm{BiFeO}_{3}$ provides great functionality for ferroelectric interface devices. ${ }^{370,373}$ In addition, with increasing film thickness, the tetragonal $\mathrm{BiFeO}_{3}$ phase partially relaxes to a pseudo-rhombohedral phase, resulting in an effective morphotropic phase boundary (MPB) where the two structures coexist (see Fig. 6). ${ }^{238,374}$ The MPB exhibits enhanced functionalities, such as enhanced piezoelectric properties and photovoltaic effect. ${ }^{375}$ The excellent ferroelectric, piezoelectric and electrooptic ${ }^{370,376}$ properties of tetragonal $\mathrm{BiFeO}_{3}$ make it of high interest as a $\mathrm{Pb}$-free ferroelectric replacement for PZT.
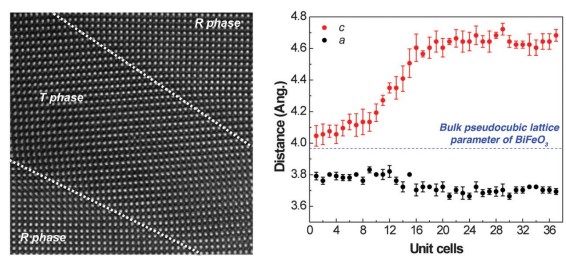

FIG. 6. Left: Cross-sectional high angle annular dark field TEM image of the boundaries between pseudo-rhombohedral $(\mathrm{R})$ and pseudo-tetragonal $(\mathrm{T})$ regions, indicated by dashed lines of a $\mathrm{BiFeO}_{3}$ film grown on an $\mathrm{LaAlO}_{3}$ substrate. Right: In-plane (black) and out of plane (red) lattice constants of $\mathrm{BiFeO}_{3}$ across the R-T boundary. From Science 326, 977 (2009). Reprinted with permission from AAAS.

\section{4. $\mathrm{HfO}_{2}$}

The recent discovery of ferroelectricity in pure and doped $\mathrm{HfO}_{2}$ ultrathin films ${ }^{173,377,378}$ is also of high interest for interfacial ferroelectric devices, given its compatibility with CMOS technology and the robust ferroelectric and dielectric properties at thicknesses in the range 
Epitaxial ferroelectric interfacial devices

from 3-30 nm, including high electric coercive fields and high breakdown voltages (see Table I). ${ }^{379,380}$ Ferroelectricity in $\mathrm{HfO}_{2}$ is associated with a metastable polar orthorhombic phase that can be stabilized by interfacial strain, growth orientation, small crystallite grain size, doping (such as with Si, Zr, Al, Y, Gd, Sr and La) or in combination with the application of an external electric field. ${ }^{173,378,381-384}$ It is a displacive ferroelectric where the electric dipole moment arises from a coordinated displacement of four of the eight oxygen ions in the orthorhombic unit cell, defining the $c$ direction of the orthorhombic structure and the direction of the ferroelectric polarization; the ferroelectric phase is chiral (upon a point rotation, the polarization reverses but the structure is not that of the mirror reflected structure), with both chiralities possessing the same energy and reversal of the polarization occurring via the coordinated displacement of the same four oxygen cations along the $c$ direction. . $^{378,381}$

Most studies on ferroelectric $\mathrm{HfO}_{2}$ reported in the literature are for polycrystalline films grown by atomic layer deposition (ALD) or by chemical solution deposition, typically on TiN contact layers and requiring an annealing step for film crystallisation after metallization, ${ }^{173,378,382,383,385,386}$ where the ferroelectric orthorhombic phase coexists with a non-polar tetragonal phase. However, a growing number of studies have reported the growth of epitaxial ferroelectric $\mathrm{HfO}_{2}$ films. ${ }^{387-398}$ One example is the epitaxial growth of 15-20 nm $\mathrm{YO}_{1.5}$-substituted $\mathrm{HfO}_{2}$ films by pulsed laser deposition on [100]-oriented yttrium oxide-stabilized zirconium oxide (YSZ) substrates, ${ }^{387}$ and on YSZ(110) single crystals using Sn-doped $\mathrm{In}_{2} \mathrm{O}_{3}$ (ITO) as a bottom electrode, ${ }^{389}$ where large ferroelectric polarizations and an estimated critical temperature of $723 \mathrm{~K}$ were observed. A cross-sectional annular bright field STEM image of a ca. $20 \mathrm{~nm}\left(\mathrm{YO}_{1.5}\right)_{0.07}\left(\mathrm{HfO}_{2}\right)_{0.93} / \mathrm{YSZ}(100)$ is shown in Fig. 7 together with simulations of the patterns expected for the polar and non-polar phases, showing a good correspondence for the polar structure. ${ }^{387}$ Most recently, first principles calculations and x-ray diffraction measurements of epitaxial single crystals grown in the [111] orientation show stabilization of a predicted ground state Pnm21 structure. ${ }^{399}$ Hence, there is promise that better control over the crystalline growth of ferroelectric $\mathrm{HfO}_{2}$ thin films could be achieved; the harnessing of its excellent dielectric and ferroelectric properties would be of high interest for the future development of interfacial ferroelectric devices. ${ }^{379,400-402}$

A related ferroelectric that has been recently discovered is monolayer $\mathrm{ZrO}_{2}$ grown on silicon. ${ }^{403,404}$ One of the stable crystal structures of $\mathrm{ZrO}_{2}$ is the fluorite structure, in which 
Epitaxial ferroelectric interfacial devices
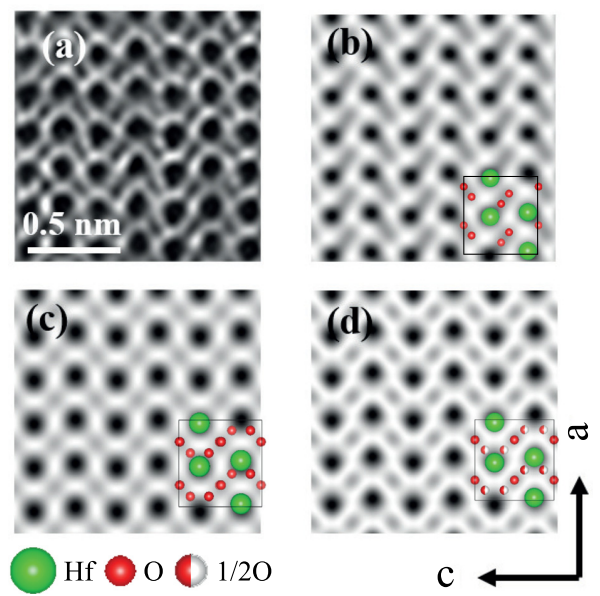

FIG. 7. (a) Cross-sectional annular bright field (ABF)-STEM image of a $20 \mathrm{~nm}$ $\left(\mathrm{YO}_{1.5}\right)_{0.07}\left(\mathrm{HfO}_{2}\right)_{0.93}$ epitaxial film grown on YSZ(100). Simulated ABF-STEM images for (b) polar Pca2 $2_{1}$, (c) non-polar Pbca, and (d) pbcm structures. Adapted from Appl. Phys. Lett. 107, 032910 (2015), with the permission of AIP Publishing.

an individual atomic $\mathrm{ZrO}_{2}$ plane in the material is polar. In the bulk, this polarization is not switchable, but a combination of first principles calculations and experiment shows that a single $\mathrm{ZrO}_{2}$ plane grown atomically abruptly on silicon has two stable structures with different polarizations. The ferroelectric structure then consists of a continuous monolayer of $\mathrm{ZrO}_{2}$ that forms on silicon when grown using molecular beam epitaxy on an atomically clean $\operatorname{Si}(001)$ surface. When the polarization is switched by application of an electric field, the atomic configuration switches and shifts the silicon surface potential by $0.6 \mathrm{~V}{ }^{404}$ Capacitance-voltage measurements of the gate stack show a large hysteresis with a direction that follows the ferroelectric switching of the $\mathrm{ZrO}_{2}$ monolayer. This new class of materials consisting of a single atomic monolayer thick represents the lower limit of device scaling.

\section{Role of the supporting substrate}

One important aspect in studying thin ferroelectric films concerns the role of the supporting substrate, whose choice has to be considered judiciously. Besides chemical stability ${ }^{245}$ 
Epitaxial ferroelectric interfacial devices

a first criterion is a relatively good lattice match in order to enable epitaxial growth, but equally important is its impact on the ferroelectric properties of the film due to the elastic and electric constraints that it imposes on the ferroelectric system. By acting as mechanical boundary condition, it can impose a strain on the ferroelectric film either due to the lattice and/or the thermal misfit associated with the growth process (respectively, lattice mismatch and difference in thermal expansion between film and substrate). Strain affects strongly the ferroelectric properties, including the critical temperature, piezoelectric constants, and ferroelectric polarization. ${ }^{12,14,405}$ For example, the ferroelectric critical temperature and polarization of strained $\mathrm{BaTiO}_{3}$ thin films can be enhanced by up to $500 \mathrm{~K}$ and $250 \%$, respectively, ${ }^{218}$ while the quantum paraelectric $\mathrm{SrTiO}_{3}$ becomes ferroelectric under tensile or compressive strains of about $1 \% .{ }^{406-408}$ The substrate also defines one electrical boundary condition, impacting the electrostatic energy of the system. In the case of an insulating substrate, the presence of a surface bound charge layer at the ferroelectric interface would result in a large electrostatic energy, which the system minimizes by breaking into ferroelectric domains, switching to an in-plane ferroelectric state, or in extreme cases, making the paraelectric state energetically more favourable. In the case of a conducting substrate, the free carriers in the latter will screen the electric field associated with the ferroelectric surface polarization; however, an electric depolarization field across the ferroelectric may still be present if the potential difference to the other surface is different from zero (open circuit condition) or if there is no potential difference (short-circuit condition). In addition, the presence of charge carriers in the ferroelectric layer induced by either point defects ${ }^{177,409-412}$ or band bending at the interface ${ }^{413}$ can result in inhomogeneous internal electric field profiles across the interface, which can manifest in an imprint field that may favor one orientation of the ferroelectric polarization over the other. ${ }^{414}$ At a more subtler level, also the particular atomic termination of the substrate may condition the growth of the ferroelectric film and give rise to preferred orientations of the ferroelectric polarization. ${ }^{153,156,160,415,416}$

\section{Elastic boundary conditions: epitaxial strain}

Typically, the growth of single crystalline films requires a single crystal substrate that serves as an atomic template for the oriented crystal growth (epitaxy). In the general case, the equilibrium lattice parameter, symmetry, and chemistry of the deposited film material 
Epitaxial ferroelectric interfacial devices

is different from that of the substrate (heteroepitaxy), such that the lattice registry imposed by minimization of the bond energy at the interface sets an effective elastic boundary condition in the form of a misfit strain. However, the condition for a coherently strained epitaxial film applies insofar as the system can accommodate the associated elastic energy, which increases linearly with film thickness. Above a critical thickness, the build up in elastic energy can be favourably counteracted by mechanisms that act to release strain, including the formation of dislocations, ${ }^{417-422}$ surface roughness, ${ }^{422-425}$ and strain-induced atomic diffusion at elevated temperatures. ${ }^{426,427}$ Hence, epitaxial film growth typically proceeds first through coherent growth, whereby the epitaxial film grows fully strained and in registry with the underlying substrate up to a critical thickness above which strain relaxation processes set in, particularly by the introduction of misfit dislocations. By equating the energy gain through strain release to the strain energy associated with the dislocation core (which involves a disruption of the local crystal structure), expressions for the critical coherence thickness can been deduced to provide estimates of the thickness above which strain starts to be relaxed (which however do not consider kinetic barriers for misfit dislocation formation, such that larger critical coherence thicknesses than those predicted from theory are often observed experimentally). ${ }^{292,428}$ For ferroelectrics, epitaxial strain has a strong impact on the ferroelectric properties, given the intimate link between lattice structure and ferroelectricity in many ferroelectric systems. ${ }^{12,14,405}$ The effect can be two-fold: for coherent strain, uniform changes in the ferroelectric properties may be expected, including changes in the ferroelectric polarization and critical temperature, while in the incoherent thickness regime, the local stresses associated with the cores of the dislocations and the associated strain gradients (such as through the flexoelectric effect) ${ }^{429-434}$ can lead to local modulations in the ferroelectric order parameter and to modified ferroelectric properties (section II E). ${ }^{312}$

The effect of coherent epitaxial strain on the properties of thin ferroelectric films has been extensively addressed in the literature. ${ }^{12,236,405}$ In the case of the perovskite ferroelectrics, the effect of biaxial strain is that of modifying the $c / a$ ratio and rotating the oxygen octahedra. ${ }^{22,435}$ Its effect on the ferroelectric polarization can be estimated to linear order in strain in terms of the improper piezoelectric tensor $c_{\alpha i}=\partial P_{\alpha} / \partial \epsilon_{i}$, where $\epsilon_{i}$ are the strain components expressed in the Voigt notation and $\alpha$ stands for the cartesian coordinate. For an in-plane biaxial strain and considering only out of plane polarization, one obtains for 
Epitaxial ferroelectric interfacial devices

the change in polarization ${ }^{236}$

$$
\Delta P_{3}=\left(2 c_{31}-\frac{c_{33}}{n}\right) \epsilon_{1}=c_{\mathrm{eff}} \epsilon_{1}
$$

where $n=-\epsilon_{1} / \epsilon_{3}$ is the Poisson ratio. The results for a number of systems $\left(\mathrm{BaTiO}_{3}, \mathrm{BiFeO}_{3}\right.$, $\mathrm{PbTiO}_{3}$, and $\mathrm{LiNbO}_{3}$ ) are shown in Fig. 8 together with the results of $a b$ initio calculations (symbols). ${ }^{236}$ It shows that the strongest variation occurs for $\mathrm{BaTiO}_{3}$ and $\mathrm{PbTiO}_{3}$, as a consequence of the large $c_{33}$ values in combination with relatively low Poisson ratios, while for the other materials the variation is more modest; one finds also no direct link between crystal structure and polarization susceptibility to in-plane biaxial strain. As expected for the perovskite ferroelectrics, biaxial in-plane compressive strain results in an increase in the $c / a$ ratio and in the ferroelectric polarization, while the opposite is the case for tensile strain. Direct experimental confirmation of enhanced ferroelectric polarization of epitaxially compressively strained films has been reported in the literature, some examples of which are listed in Table II, showing critical ferroelectric temperatures and ferroelectric polarizations well in excess of the bulk value (Table I). For rhombohedral $\mathrm{BiFeO}_{3}$, the change in ferroelectric polarization has been observed to be largely independent of the average in-plane strain (i.e., below and above the critical thickness for coherent growth), ${ }^{428,436}$ in agreement with the theory predictions, ${ }^{236,437}$ and a similar behavior was reported for $\mathrm{Pb}\left(\mathrm{Zr}_{0.2} \mathrm{Ti}_{0.8}\right) \mathrm{O}_{3},{ }^{438}$ where the saturation ferroelectric polarization is found to be identical for both a strained $30 \mathrm{~nm}$ film $(c / a=1.09)$ and a relaxed $100 \mathrm{~nm}$ film $(c / a=1.05)$, an effect that has been ascribed to a suppressed sensitivity of the A-site cations to the epitaxial strain. ${ }^{438}$

Epitaxial strain can also induce a phase transition to a distinct supertetragonal structure (i.e., with a value of $c / a$ much larger than the bulk value and generally found to exceed 1.2) that may also have a large polarization. Two particular reports are striking, namely, for supertetragonal $\mathrm{PbTiO}_{3}$ and $\mathrm{BiFeO}_{3}$, where ferroelectric polarizations of $236 \mu \mathrm{C} / \mathrm{cm}^{2}$ and $130 \mu \mathrm{C} / \mathrm{cm}^{2}$, respectively, have been reported, ${ }^{240,439}$ consistent with first principles calculations. ${ }^{234,440,441}$

In addition to modifying the ferroelectric polarization, epitaxial strain also affects the ferroelectric critical temperature by hindering the transition to the higher symmetry paraelectric phase, while the order of the phase transition may change from first to second order as a consequence of the reduced electrostrictive coupling in strained ferroelectric films; ${ }^{442-444}$ intuitively speaking, epitaxial prevents the system to lower the crystal symmetry to cubic 
Epitaxial ferroelectric interfacial devices

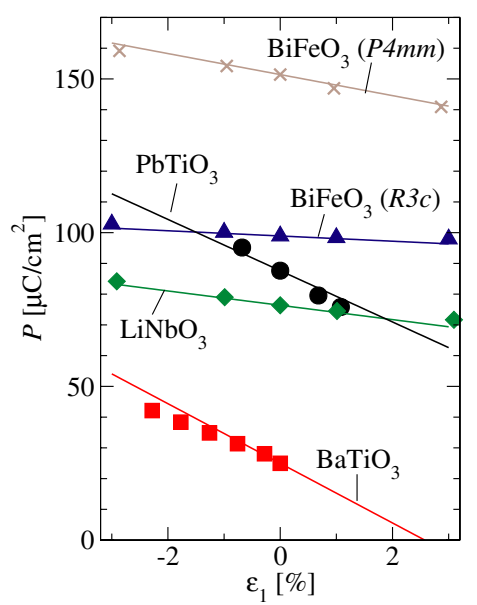

FIG. 8. Variation of the spontaneous ferroelectric polarization $P$ for several ferroelectrics vs. epitaxial strain $\epsilon_{1}$ estimated from expression 1 (lines) and from first principles calculations (symbols). Reproduced with permission from Phys. Rev. Lett. 95, 257601 (2005). Copyright 2005 by the American Physical Society.

across the phase transition, resulting in a continuous change in the order parameter. In addition, epitaxial strain can induce the formation of new crystalline phases that otherwise could not be stabilized in bulk. ${ }^{12,218,405,443,445}$ Phenomenologically, epitaxial strain adds an electrostrictive contribution to the polarization that modifies the onset of ferroelectricity in the film; for a [001]-oriented thin film, the change in critical temperature for an out of plane polarized film (tetragonal $c$ phase) is estimated as: ${ }^{443}$

$$
T_{3}=T_{0}+2 C \epsilon_{0} \frac{2 Q_{12}}{s_{11}+s_{12}} u_{m}
$$

while for the onset of in-plane polarization along the $\langle 110\rangle$ direction (orthorhombic a a phase):

$$
T_{1}=T_{0}+2 C \epsilon_{0} \frac{Q_{11}+Q_{12}}{s_{11}+s_{12}} u_{m},
$$

where $T_{0}$ is the bulk critical temperature, $C$ is the Curie constant, $\epsilon_{0}=8.854187817 \times$ $10^{-12} \mathrm{~F} / \mathrm{m}$ is the electric permittivity of free space, $Q$ are the electrostrictive coefficients, $s$ are the elastic compliances, and $u_{m}=\left(a_{s}-a_{0}\right) / a_{0}$ is the misfit strain, where $a_{s}$ is the substrate lattice parameter and $a_{0}$ the equilibrium lattice parameter of the free standing 
Epitaxial ferroelectric interfacial devices

film. The above expressions predict that, when $Q_{12}<0$ and $Q_{11}+Q_{12}>0$, as is the case for $\mathrm{BaTiO}_{3}$ and $\mathrm{PbTiO}_{3}$, the critical temperature increases (linearly) for both signs of the misfit strain. ${ }^{443}$ Examples of the effect of epitaxial strain in enhancing the critical temperature are given in Table II for several perovskite ferroelectrics, showing a significant increase in $T_{\mathrm{c}}$ with respect to their bulk values (Table I). The enhanced ferroelectric polarization and critical temperature induced by epitaxial strain are beneficial for ferroelectric interfacial devices, since it is conducive to larger field effects over a larger temperature range (as far as the ferroelectric component is concerned). In $\mathrm{BiFeO}_{3}$, the presence of both polar displacements and oxygen octahedra rotations yields an anomalous strain dependence of the Curie temperature. It actually decreases with increasing strain, which is captured by effective hamiltonian calculations. ${ }^{446}$

TABLE II. Ferroelectric characteristics of epitaxially strained thin ferroelectric films. $t$ is the film thickness, $\epsilon_{1}$ the in-plane strain (when not available, the room temperature lattice misfit is given instead in square brackets); PLD: pulsed-laser deposition, RFS: r.f. sputtering.

\begin{tabular}{|c|c|c|c|c|c|c|c|}
\hline System & $\begin{array}{l}t \\
(\mathrm{~nm})\end{array}$ & $\begin{array}{l}\epsilon_{1} \\
(\%)\end{array}$ & $c / a$ & $\begin{array}{c}P_{\mathrm{s}} \\
\left(\mu \mathrm{C} / \mathrm{cm}^{2}\right)\end{array}$ & & $\begin{array}{l}\text { Growth } \\
\text { technique }\end{array}$ & Ref. \\
\hline $\mathrm{SRO} / \mathrm{BaTiO}_{3} / \mathrm{SRO} / \mathrm{GdScO}_{3}(110)$ & 200 & -1.0 & 1.026 & 50 & 693 & PLD & 218 \\
\hline $\mathrm{SRO} / \mathrm{BaTiO}_{3} / \mathrm{SRO} / \mathrm{DyScO}_{3}(110)$ & 200 & -1.3 & 1.031 & 70 & 953 & PLD & 218 \\
\hline $\mathrm{PbTiO}_{3} / \mathrm{SrTiO}_{3}(001)$ & 130 & {$[-0.06]$} & 1.05 & - & $>973$ & PLD & 292 \\
\hline $\mathrm{Pt} / \mathrm{PbTiO}_{3} / \mathrm{Nb}: \mathrm{SrTiO}_{3}(100)$ & 129 & {$[-0.06]$} & 1.238 & 236 & $>998$ & RFS & 240 \\
\hline $\mathrm{SRO} / \mathrm{PbZr}_{0.2} \mathrm{~Pb}_{0.8} \mathrm{TiO}_{3} / \mathrm{SRO} / \mathrm{SrTiO}_{3}(001)$ & 90 & {$[-0.7]$} & 1.06 & 105 & - & PLD & 186 \\
\hline $\mathrm{PbZr}_{0.2} \mathrm{~Pb}_{0.8} \mathrm{TiO}_{3} / \mathrm{Nb}-\mathrm{SrTiO}_{3}(001)$ & 15 & -1.2 & 1.07 & - & 953 & RFS & 313 \\
\hline $\mathrm{Pt} / \mathrm{BiFeO}_{3} / \mathrm{LSMO} / \mathrm{LaAlO}_{3}(100)$ & 160 & {$[-1.0]$} & 1.24 & $130 \pm 5$ & - & PLD & 439 \\
\hline
\end{tabular}

While the role of a uniform strain is largely beneficial and can be employed to tune the characteristics of ferroelectric thin films, strain relaxation in thin films is expected to have a predominantly negative impact on the ferroelectric properties of thin films. ${ }^{312}$ For example, calculations based on a thermodynamic analysis predict strong variations in the local ferroelectric polarization near the core of the dislocations that lead to strong depolarization fields that suppress ferroelectricity in a region that can extend over several nanometers from the interface $^{447-449}$ A telling example is the local reduction of the ferroelectric polarization of a 
Epitaxial ferroelectric interfacial devices

PZT film by up to $48 \%$ caused by the strain field of a dislocation inside an adjacent $\mathrm{SrTiO}_{3}$ layer, determined from TEM. ${ }^{450}$ The presence of such a depolarization layer can lead to the formation of ferroelectric dead layers that absorb a large fraction of the applied electric field and that can act as pinning centers for the ferroelectric domain walls. ${ }^{451}$ The negative role of misfit dislocations has been identified in several systems, including PZT nanoislands ${ }^{452}$ and thin PZT films. ${ }^{453-456}$

Another mechanism for strain relaxation in thin ferroelectric films is through domain formation, such as domains with either the $a$-axis or $c$-axis oriented perpendicular to the surface ( $a$ and $c$ domains) in tetragonal ferroelectric systems. ${ }^{457-459}$ Also for this case, a critical thickness has been estimated above which it is energetically favourable for the system to develop a mixed domain configuration. A manifestation of this process can be found in thick $c$-oriented PZT films, where a significant $a$-domain population is often observed experimentally. ${ }^{145,456,460}$ The presence of $a$-domains is undesirable, as they lower the switchable out of plane ferroelectric polarization of the system and contribute to inhomogeneities that are detrimental to device scaling. ${ }^{458}$

\section{Electric boundary conditions and size effects}

The substrate also sets an electrical boundary condition for the ferroelectric film, with important consequences for the equilibrium ferroelectric configuration of the system. This is due to the role of the electrostatic energy, which, in the absence of free charge, is minimized for zero divergence of the ferroelectric polarization $(\nabla \cdot \mathbf{P}=0)$ and no net bound charge at the boundaries ( $\mathbf{n} \cdot \mathbf{P}=0$, where $\mathbf{n}$ is the surface unit vector). For thin films, this is achieved for a uniform in-plane configuration of the ferroelectric polarization. However, since the direction of the latter is tied to the lattice, the ferroelectric polarization in epitaxial ferroelectric thin films is not free to orient along the direction that minimizes the electrostatic energy. In fact, for field effect devices, a uniform orientation of the ferroelectric polarization along the direction normal to the film plane is generally the most desirable configuration. The latter can be achieved by constraining the polar axis to be oriented along the out of plane direction, as in [001]-oriented tetragonal ferroelectric systems or in rhombohedral [001]oriented $\mathrm{BiFeO}_{3}\left([111]_{\mathrm{ps}}\right.$ of the pseudo-cubic perovskite structure); also in rhombohedral $\mathrm{BiFeO}_{3}$ grown on $\mathrm{SrTiO}_{3}(001)$, the polarization makes an angle of about $36^{\circ}$ from the surface, 
Epitaxial ferroelectric interfacial devices

resulting in a large polarization component along the out of plane direction. ${ }^{355,364,365,461}$ In case the ferroelectric polarization cannot be screened by free charges (such as provided by a conducting substrate and/or polar molecules at the free surface), ${ }^{413,462,463}$ the electrostatic energy of the system can be minimized by the formation of oppositely poled ferroelectric domains, ${ }^{127,129,464,465}$ or by reverting to the paraelectric state at the ultrathin limit. ${ }^{466-468}$

The geometry of ferroelectric interfacial devices is typically that of a capacitor structure, where the ferroelectric layer is sandwiched between two conducting layers, for example, a gate contact and a channel layer in the case of ferroelectric field effect devices. These layers provide not only for device function, but act as well to screen the ferroelectric polarization at the respective interface and ensure the required stability for the uniformly polarized ferroelectric state. However, at metallic interfaces, screening occurs over a finite length scale of the order of the characteristic Thomas-Fermi screening length, $\delta_{\mathrm{TF}} \sim 0.339\left(r_{s} / a_{0}\right)^{1 / 2}$ (where $r_{s}=(3 / 4 \pi n)^{1 / 3}$ is the mean distance between carriers, $n$ the carrier density, and $a_{0}=0.529177 \AA$ the Bohr atomic radius). ${ }^{469}$ Over this screening length the electric field associated with the ferroelectric polarization is non-zero, therefore acting as a dielectric layer of thickness ${ }^{465} d \sim 2 \delta_{\mathrm{TF}}$ that adds to the electrostatic energy and contributes to a series capacitance that absorbs a fraction of the applied electric field. In fact, the depolarizing field associated with the screening length of the metallic contact can be sufficient to destabilize the ferroelectric order of ultrathin films, setting a limit to the minimum thickness required for the onset of ferroelectricity that depends on electrode material, on the ionic polarizability, and on the ferroelectric band structure. ${ }^{34,466,467}$ For example, in $\mathrm{PbTiO}_{3}$ and $\mathrm{BaTiO}_{3}$, theoretical ab initio studies have predicted the onset of ferroelectricity at 1 and 6 unit cells, respectively. ${ }^{34,466,467}$ Experimentally, ferroelectricity has been confirmed in $\mathrm{BaTiO}_{3}, \mathrm{PbTiO}_{3}, \mathrm{~Pb}\left(\mathrm{Zr}_{0.2} \mathrm{Ti}_{0.8}\right) \mathrm{O}_{3}, \mathrm{BiFeO}_{3}, \mathrm{HfO}$, and $\mathrm{KNbO}_{3}$ films down to a few unit cells in thickness (Table III).

For device applications, it is important to be able to apply electric fields, for example, to switch the direction of the ferroelectric polarization. Since most oxide ferroelectrics have relatively modest band gaps $(\sim 3 \mathrm{eV}$, see Table I) and a significant density of extrinsic charge carriers (originating typically from point defects as a consequence of the complex defect chemistry of $3 \mathrm{~d}$ metal oxides, where the metal cations can exist in various oxidation states), ${ }^{412,476-478}$ the electrical character of the contact (ohmic or diode-like) will depend on the difference between metal work function $\phi$ and the electron affinity of the ferroelectric $\chi$ 
Epitaxial ferroelectric interfacial devices

TABLE III. Experimental minimum thickness $t_{c}$ for the onset of ferroelectricity in selected ferroelectric systems.

\begin{tabular}{|c|c|c|c|c|c|c|}
\hline Material & Electrode & Substrate & Technique & $T(\mathrm{~K})$ & $t_{c}(\mathrm{~nm})$ & Ref. \\
\hline $\mathrm{BaTiO}_{3}$ & $\mathrm{SrRuO}_{3}$ & $\mathrm{SrTiO}_{3}(001)$ & P-E & $\mathrm{RT}$ & 5 & 199,470 \\
\hline $\mathrm{BaTiO}_{3}$ & $\mathrm{SrRuO}_{3}$ & $\mathrm{SrTiO}_{3}(001)$ & PFM, STEM & $\mathrm{RT}$ & 1.4 & 471 \\
\hline $\mathrm{BaTiO}_{3}$ & $\mathrm{LaAlO}_{3} / \mathrm{SrTiO}_{3}$ & $\mathrm{SrTiO}_{3}(001)$ & PFM & RT & 1.0 & 472 \\
\hline $\mathrm{PbTiO}_{3}$ & - & $\mathrm{SrTiO}_{3}(001)$ & XRD & 550 & 3 u.c. & 127 \\
\hline $\mathrm{PbTiO}_{3}$ & $\mathrm{SrRuO}_{3}$ & $\mathrm{SrTiO}_{3}(001)$ & XRD & 700 & 1.2 & 462 \\
\hline $\mathrm{PZT}$ & $\mathrm{Nb}-\mathrm{SrTiO}$ & $\mathrm{Nb}_{-} \mathrm{SrTiO}_{3}(001)$ & PFM & $\mathrm{RT}$ & 4.0 & 473 \\
\hline $\mathrm{BiFeO}_{3}$ & $\mathrm{Pt}, \mathrm{LSMO}$ & $\mathrm{SrTiO}_{3}(001)$ & PFM & RT & 1.6 & 474 \\
\hline $\mathrm{HfO}$ & $\mathrm{p}^{+}-\mathrm{Ge}$ & $\mathrm{p}^{+}-\mathrm{Ge}$ & P-E & $\mathrm{RT}$ & 3 & 380 \\
\hline $\mathrm{KNbO}_{3}$ & - & $\mathrm{SrTiO}_{3}(001)$ & STEM & $\mathrm{RT}$ & 4 & 475 \\
\hline
\end{tabular}

(which defines the height of the Schottky barrier, $V_{\mathrm{bi}}=\phi-\chi$ ), the charge carrier density in the ferroelectric (which defines the width of the depletion layer) and the presence of interface states (metal-induced gap states or interface defect states), which may pin the position of the Fermi level of the ferroelectric charge carriers. ${ }^{171,476-481}$ The electrostatic physics is illustrated in Fig. 9, showing the band alignment for a perfectly insulating ferroelectric in contact with identical metal electrodes compared to that of a defective ferroelectric layer. The situation is similar to that of a metal/semiconductor contact, where the role of the ferroelectric polarization is that of adding a sheet of bound charge at the interface to the electrostatic configuration. ${ }^{476,479,482}$ To account for the presence of a depletion layer in the metallic contact or a ferroelectric dead layer, a thin dielectric layer of thickness $\delta$ is added between the ferroelectric and the metal, which is otherwise treated as ideal. To first approximation, the ferroelectric polarization adds a term to the Schottky built-in potential, $V_{\mathrm{bi}},{ }^{476,479,482}$

$$
V_{\mathrm{bi}}^{\prime}=V_{\mathrm{bi}} \pm P \delta /\left(\epsilon_{0} \epsilon_{\mathrm{s}}\right)
$$

where $P$ is the ferroelectric polarization amplitude, $\delta$ the width of the dielectric layer, $\epsilon_{\mathrm{s}}$ its static dielectric constant, and the \pm sign corresponds to positive or negative directions of the ferroelectric polarization. For $P \sim 50 \mu \mathrm{C} / \mathrm{cm}^{2}, \delta \sim 0.2 \AA$, and $\epsilon_{\mathrm{s}} \sim 2$, the additional contribution to the built-in potential is of the order of $0.5 \mathrm{~V}$, which is considerable. In fact, the 
Epitaxial ferroelectric interfacial devices

experimental determination of the polarization dependence of the Schottky barrier height has been reported for several systems, including $\mathrm{BaTiO}_{3}, \mathrm{BiFeO}_{3}$ and $\mathrm{Pb}\left(\mathrm{Zr}_{0.2} \mathrm{Ti}_{0.8}\right) \mathrm{O}_{3}$, showing indeed that such large variations in the Schottky barrier height are reached, see Table IV. In some instances, the change in the Schottky barrier may be sufficiently high to drive the contact from diode-like to ohmic, leading to large changes in the conductivity of the system when switching the direction of the ferroelectric polarization. ${ }^{104,483-486}$ A similar interface structure will be present at the other side of the ferroelectric layer, possibly with a different conducting material. Hence, the requirement for being able to apply an electric field of sufficient amplitude for switching the ferroelectric polarization is that both interfaces consist of back-to-back Schottky diode junctions. The presence of charge carriers, either intrinsic or due to extrinsic doping arising from point defects, show that ferroelectrics for the most part are not perfect insulators, but instead exhibit charge transport under an applied electric field. In addition to thermally activated transport of charge carriers across the Schottky barrier, and in common to other insulators, also tunneling in ultrathin films, electron hopping, or emission from deep traps are also found to contribute to the conductivity; ${ }^{411,487}$ for ferroelectrics, it has been recently discovered that ferroelectric domain walls tend to exhibit significantly different electronic properties as compared to the bulk material, ${ }^{488,489}$ including high electric conductivity. ${ }^{490-495}$

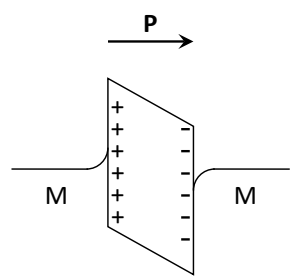

(a)

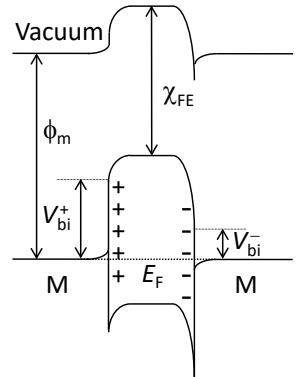

(b)

FIG. 9. (a) Schematic of the band alignment at the metal contact/ferroelectric interface for a perfectly insulating ferroelectric. (b) For an imperfectly insulating ferroelectric, the presence of mobile charges results in band bending to screen the potential associated with the difference in the Fermi levels of the metal and ferroelectric (including the interfacial bound charge). 
Epitaxial ferroelectric interfacial devices

Different types of mobile charge carriers may be involved in screening the ferroelectric polarisation at the interface. ${ }^{41,414}$ For example, a detailed STEM-EELS study by Kim et al. ${ }^{496}$ of the $\mathrm{BiFeO}_{3} / \mathrm{La}_{0.8} \mathrm{Sr}_{0.2} \mathrm{MnO}_{3}$ interface in a region of the sample containing two ferroelectric domains with opposite polarization shows that the domain with the ferroelectric polarization pointing away from the interface presents an out of plane lattice expansion of $\sim 5 \%$ in the $\mathrm{BiFeO}_{3}$ interface region, together with a decrease in the Mn valency and a reduction in the O K edge intensity, which is interpreted as due to screening by oxygen vacancies; in contrast, the other polarization direction shows no such effects and screening is inferred to be purely electronic. $^{496}$

TABLE IV. Variation of the Schottky barrier height for ferroelectric/metal contacts for the two directions of the ferroelectric polarization, pointing into $\left(\phi^{+}\right)$or away $\left(\phi^{-}\right)$from the metal interface. $\Delta \phi=\phi^{+}-\phi^{-}$, and $t$ is the thickness of the ferroelectric. XPS: x-ray photoemission spectroscopy; STS: scanning transmission spectroscopy.

\begin{tabular}{|c|c|c|c|c|c|c|}
\hline Interface & $t(\mathrm{~nm})$ & $\phi^{+}(\mathrm{eV})$ & $\phi^{-}(\mathrm{eV})$ & $\Delta \phi(\mathrm{eV})$ & Technique & Ref. \\
\hline $\mathrm{BaTiO}_{3} / \mathrm{RuO}_{2}$ & bulk & $2.85 \pm 0.15$ & $1.75 \pm 0.15$ & $1.1 \pm 0.3$ & XPS & 481 \\
\hline $\mathrm{BaTiO}_{3} / \mathrm{Pt}$ & bulk & $2.90 \pm 0.15$ & $2.25 \pm 0.15$ & $0.65 \pm 0.3$ & XPS & 481 \\
\hline $\mathrm{BaTiO}_{3} / \mathrm{Pt}$ & 64 & 0.40 & 0.85 & 0.45 & XPS & 486 \\
\hline $\mathrm{BaTiO}_{3} / \mathrm{Nb}-\mathrm{SrTiO}_{3}$ & 64 & 0 & 0.45 & -0.45 & XPS & 486 \\
\hline $\mathrm{BaTiO}_{3} / \mathrm{Cr}$ & 3 & 0.31 & 0.34 & -0.03 & XPS & 497 \\
\hline $\mathrm{BaTiO}_{3} / \mathrm{Pt}$ & 3 & 0.42 & 1.84 & -0.38 & XPS & 497 \\
\hline $\mathrm{BaTiO}_{3} / \mathrm{LSMO}$ & 2.8 & 1.35 & 1.05 & 0.3 & XPS & 134 \\
\hline PZT/LSMO & 4 & 0.98 & 0.25 & 0.7 & XPS & 498 \\
\hline $\mathrm{BiFeO}_{3} / \mathrm{Nb}-\mathrm{SrTiO}_{3}$ & 120 & $1.0 \pm 0.1$ & $0.2 \pm 0.1$ & $0.8 \pm 0.2$ & STS & 499 \\
\hline
\end{tabular}

In addition to screening by mobile charges discussed above, other mechanisms can also contribute to screen the ferroelectric polarization, including ionic screening. For example, Gerra et al. ${ }^{500}$ suggested in their theoretical work that additional ionic compensation to the depolarization field can occur at a ferroelectric/electrode interface. By considering a ferroelectric $\mathrm{BaTiO}_{3}$ layer sandwiched between $\mathrm{SrRuO}_{3}$ electrodes as a model system, they show that the ferroelectric ionic displacement of the ferroelectric $\mathrm{BaTiO}_{3}$ layer may penetrate for several atomic layers into the $\mathrm{SrRuO}_{3}$ electrode. The induced ionic displacement inside the 
Epitaxial ferroelectric interfacial devices

electrode acts to further stabilize ferroelectricity by pushing the center of mass of free electrons towards the interface. Subsequent theoretical and experimental work has shown that ionic compensation is active in various ferroelectric/metal interfaces and is crucial in stabilizing ferroelectricity down to the nanoscale. ${ }^{52,467,471,501}$ Conversely, the ferroelectric induced ionic displacement inside the electrode can result in changes in its electronic properties, leading to an additional mechanism for controlling the correlated state of the conducting channel (see section IV).

Although we have focused on the impact on the ferroelectric properties of materials with decreasing film thickness down to the atomic scale, additional considerations come to the fore when also the lateral dimension of the ferroelectric system is reduced to the nanoscale, in particular for free standing nanoparticle systems, where new domain states such as flux closure states can occur to minimise the electrostatic energy and a critical size may be required to sustain a single domain state and at smaller dimensions, to sustain a stable ferroelectric state against thermal excitations. ${ }^{502-505}$

\section{Atomic bonding effects}

In addition to the constraints imposed by elastic and electrostatic constraints, atomic bonding effects arising from electron exchange at the interface with the substrate or top layer can have an important effect on the overall properties of the system that are important to consider. The study of such phenomena often requires probing the local electronic structure at the interface, and in this context, the recent advances in high resolution TEM have been invaluable. ${ }^{28,144}$ One aspect concerns the chemical bonding between the ferroelectric layer and the adjacent conducting channel, which may impose restrictions on the ferroelectric soft-mode across the heterointerface and affect significantly the ferroelectric properties of the system. ${ }^{52,506}$ In addition, the asymmetric environment at the interface can induce changes in the electronic state and a concomitant modification of the ferroelectric energy landscape. A striking example of this effect is a preference for a particular orientation of the ferroelectric polarization (imprint effect) that is found to depend on the termination of the interface layer, for example, in $\mathrm{BiFeO}_{3} / \mathrm{LSMO}^{415} \mathrm{PZT} / \mathrm{LSMO},{ }^{156,416}$ and $\mathrm{BaTiO}_{3} / \mathrm{LSMO}{ }^{160}$

In extreme cases, the presence os such in-built bias potential can lead to back-switching of ferroelectric domains and loss of information. ${ }^{146,507}$ The role of interface termination and 
Epitaxial ferroelectric interfacial devices

of the energy associated with the polar discontinuity ${ }^{508-511}$ on the ferroelectric state has been studied for several perovskite interfaces. ${ }^{415}$ For example, in ferroelectric $\mathrm{BiFeO}_{3}$ and metallic $\mathrm{La}_{0.7} \mathrm{Sr}_{0.3} \mathrm{MnO}_{3}$ heterostructures, the choice of the interface termination sequence, $(\mathrm{BiO})^{+} /\left(\mathrm{MnO}_{2}\right)^{-0.7}$ or $\left(\mathrm{FeO}_{2}\right)^{-} /\left(\mathrm{La}_{0.7} \mathrm{Sr}_{0.3} \mathrm{O}\right)^{+0.7}$, induces a different charge valence mismatch, +0.3 or -0.3 , at the interface, which results in the formation of an interface dipole that affects the polarization switching of the $\mathrm{BiFeO}_{3}$ layer. ${ }^{153,415}$ The interface dipole can be formed even when no charge valence mismatch is expected. In the $\mathrm{BaO} / \mathrm{RuO}_{2}$ interface termination at $\mathrm{BaTiO}_{3} / \mathrm{SrRuO}_{3}$, the difference in ionic radii of the cations induce a pinned interface dipole that hinders ferroelectric switching of the system. ${ }^{512,513}$ The undesirable interface dipole effect can be avoided by changing the $\mathrm{BaO} / \mathrm{RuO}_{2}$ termination to $\mathrm{TiO}_{2} / \mathrm{SrO}$ or by inserting a few atomic layers of $\mathrm{SrTiO}_{3} \cdot{ }^{471,514}$ Such results emphasize the importance of the proper choice of the interface terminating sequence in designing electronic devices based on nanoscale ferroelectricity. Another effect pertains to atomic-scale structural distortions at the interface layers, not only in terms of uniform epitaxial strain, but also associated with modifications in the oxygen octahedral rotations characteristic of some ferroelectric systems, such as $\mathrm{BiFeO}_{3}$. For example, Kim et al. ${ }^{154}$ have reported the onset of a non-polar $\mathrm{BiFeO}_{3}$ interface layer at the $\mathrm{La}_{0.8} \mathrm{Sr}_{0.2} \mathrm{MnO}_{3}$ interface of $\mathrm{BiFeO}_{3} / \mathrm{La}_{0.8} \mathrm{Sr}_{0.2} \mathrm{MnO}_{3} / \mathrm{SrTiO}_{3}(001)$ films, whereby a $\mathrm{MnO}_{2}$ termination of the $\mathrm{La}_{0.8} \mathrm{Sr}_{0.2} \mathrm{MnO}_{3}$ leads to a suppression of the octahedral rotation angle at the interface extending up to three atomic layers and whose effect propagates over a $3 \mathrm{~nm}$ region, while the LaSrO termination leads also to a reduced octahedral rotation compared to the bulk that extends to the whole of the film, as shown in Fig. 10. As a consequence of the presence of the non-polar $\mathrm{BiFeO}_{3}$ interface layer, the piezoelectric properties of the $\mathrm{MnO}_{2}$ terminated film are found to be strongly reduced as compared to the other termination.

\section{E. Impact of defects}

We focus in this review on metal oxide materials, for which the lattice parameter is largely determined by the oxygen sublattice and the oxygen ionic radius (in the range from 1.35 to $1.42 \AA$, increasing with increasing coordination number) $;^{515,516}$ hence, the growth of oxides on oxides is facilitated by both chemical compatibility and the relatively small lattice mismatches. In particular, we have highlighted ferroelectric systems where highly ordered single 
Epitaxial ferroelectric interfacial devices
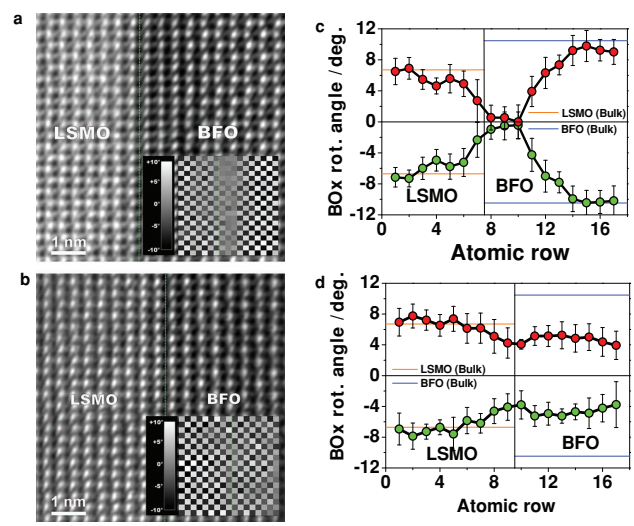

FIG. 10. Octahedral tilt and polarization mapping: bright field STEM images taken in optimal conditions for oxygen column contrast in the $[110]_{\mathrm{ps}^{-}}$oriented $\mathrm{BiFeO}_{3}$ film structures grown on: a) $\mathrm{MnO}_{2}$-terminated LSMO and b) (La,Sr)O-terminated LSMO. The resulting octahedral tilt maps (insets) show a checkerboard pattern of tilt angles. (c,d) Line profiles of octahedral tilts averaged over vertical rows of the respective tilt maps in (a) and (b). Solid lines in each graph represent tilt angles in the bulk $\mathrm{BiFeO}_{3}$ and LSMO. Reproduced with permission from Adv. Mater. 25, 2497 (2013). Copyright 2013 WILEY-VCH Verlag GmbH \& Co. KGaA, Weinheim.

crystalline films can be obtained on a number of different substrate materials. However, we have also remarked that the oxide growth process is often associated with the creation of defects due to misfit strain relaxation processes (including the onset of dislocations, film roughening, and domain formation), local chemical reactivity at the interface, or associated with the oxygen defect chemistry in oxides, which is largely a direct consequence of the multiple valence states of the $3 \mathrm{~d}$ transitions metals. ${ }^{476-478}$ By locally altering the strain field, by disturbing the local electronic structure, or by adding free carriers to the system, the presence of defects can affect strongly (mostly negatively) the properties of thin ferrolectric films, including by depressing the amplitude of the local ferroelectric polarization, increasing the coercive field, lowering the breakdown voltage and inducing current leakage, charge traps, and fatigue. ${ }^{8,451,517,518}$ Since it may not be possible to fully eliminate the presence of defects, it is important to understand their role on the properties of ferroelectric thin films, and their impact on interfacial properties of ferroelectric devices, in order to determine which 
Epitaxial ferroelectric interfacial devices

ones are more deleterious for a given application.

The mechanisms that lead to the presence of defects are directly linked to the growth process itself. The growth temperature of most ferroelectric films, in the range from 500$700^{\circ} \mathrm{C}$, tends to be well above the bulk Curie temperature and it is generally assumed that film growth starts from the paraelectric cubic phase (although, as shown in Table II, under misfit strain, ferroelectricity may be present, or start to develop already at such temperatures above a certain critical thickness). ${ }^{292,313,519-521}$ At this elevated temperature, the lattice misfit is generally different from that at room temperature, ${ }^{312,522}$ and above a critical thickness, elastic energy is relieved by the onset of misfit dislocations, whose nucleation is expected to be facilitated by the available thermal energy. ${ }^{417-421,458,522}$ Strain can also make the surface unstable against deformation, whereby elastic energy is reduced by surface roughening at a cost of surface energy. ${ }^{424,425,523,524}$ As the temperature of the film is reduced to ambient temperature after growth, the density of misfit dislocations may change to reduce the thermal misfit (difference in thermal expansion coefficients) for films above the critical thickness for coherent growth and, as the thermal energy for misfit movement becomes insufficient to reach equilibrium, a residual strain may be present in the system at ambient temperature. Finally, the transition to the ferroelectric state can result in the formation of ferroelectric domains, for example, $a$-domains in $c$-oriented tetragonal ferroelectric films, as another channel for misfit strain relaxation. ${ }^{451,457-459,525,526}$

Because of the relatively high Peierls energy in the perovskites (for $\mathrm{SrTiO}_{3}$ it is estimated to be of the order or $0.5 \mathrm{eV}$ for $\langle 110\rangle\{110\}$ edge dislocations $),{ }^{527}$ gliding of dislocations is increasingly suppressed as the temperature is reduced to room temperature, such that the dislocation network observed at low temperature can be assumed to have formed during film growth. ${ }^{451,522}$ A typical consequence of this kinetic barrier is the observation of strain opposite to that expected from the lattice mismatch at room temperature due to thermal misfit between film and substrate. ${ }^{205,528}$ The particular misfit dislocation pattern in thin films can be quite complex. For the cubic perovskites, screw and edge dislocations along $\langle 110\rangle$ within $\{110\}$ glide planes, with a Burgers vector $(a / 2)\langle 110\rangle$, are energetically more favourable, but dislocations along other directions may also occur at elevated temperatures; ${ }^{529-531}$ in thin epitaxial films, only dislocations with Burgers vector parallel to the interface act to effectively reduce strain, although experimentally both edge and screw dislocations are observed. ${ }^{205,312,454}$ For example, in thick, [001]-oriented $\mathrm{BaTiO}_{3}$ films, one 
Epitaxial ferroelectric interfacial devices

observes not only a criss-cross network of misfit dislocations, but also perpendicular screw dislocations and oblique screw dislocations in the $\{110\}$ glide plane; ${ }^{205,532}$ these are thought to originate at point defects at the surface that then glide towards the interface, where they dissociate to form interfacial dislocation lines along $\langle 100\rangle$ to relieve epitaxial strain, with the perpendicular component eventually propagating to the surface and eliminated there. ${ }^{205,533}$ Strategies for minimizing the formation of misfit dislocations include increased film deposition rates, ${ }^{243,534}$ annealing the film after growth, ${ }^{535}$ and employing well conditioned single terminated substrates ${ }^{312}$ for example. Given the high internal stresses associated with the dislocation core and the direct coupling between internal stress and the ferroelectric order parameter, misfit dislocations result in strong local depolarization fields that modify the amplitude of the local polarization; in thin films with a high density of misfit dislocations, such depolarization fields may result in suppressed ferroelectric polarization (ferroelectric dead layers) and in pinning centers for domain wall motion; ${ }^{145,312,447-449,451,453-455}$ they can also act as preferential sites for atomic diffusion processes, such as oxygen transport (the impact of point defects is discussed below). ${ }^{536-538}$ This illustrates the point that different types of defects can interact in complex ways; for instance, strain fields arising from structural defects can lead to modifications to the local composition ${ }^{145,450,539}$ and promote atomic diffusion, ${ }^{426}$ while misfit dislocations can drive interdiffusion at the interface ${ }^{427}$ or lead to surface roughening and provide preferential nucleation sites for film growth. ${ }^{540}$ Strain gradients, originating from the stress fields issuing from dislocations or from compositional variations with thickness generated during film growth, can also result in depressed properties due to coupling with the ferroelectric polarisation via the flexoelectric effect ${ }^{429-432}$ that acts as an additional local internal electric field and that provides another mechanism for the presence of imprint effects (seen as an horizontal shift in the $P-E$ hysteresis loop) in defective films; however, such effect has been employed recently to bias the direction of the ferroelectric polarisation and therefore to control the polarisation state by suitable control of the growth process and film thickness. ${ }^{433,434,541,542}$

The formation of structural polydomains at the transition from the paramagnetic to ferromagnetic phase as the film is cooled down to room temperature from the temperature of deposition is another mechanism for reducing misfit strain energy. ${ }^{451,457-459,525,526,543}$ Although the process here is driven by elastic energy considerations, ${ }^{544}$ the link between structure and direction of ferroelectric polarization implies that the system will break into a 
Epitaxial ferroelectric interfacial devices

ferroelectric multidomain state as well, for example, $a$ and $c$ domains in the tetragonal ferroelectric perovskites, or a more complex domain structure for the rhombohedral perovskites, such as $\mathrm{BiFeO}_{3}$, where four variant domains can be present in thin films. ${ }^{365,461,545}$ For the tetragonal perovskites, the presence of $a$ domains leads to a reduction of the switchable ferroelectric polarization, ${ }^{460}$ since the electric fields required to fully switch an $a$ domain to a $c$ domain, either by coherent rotation or by domain wall displacement (typically strongly pinned at defects such as dislocations), ${ }^{145,152,451}$ are much higher than those required to switch the polarization along the $c$ direction. For example, in $c$-oriented PZT films, it is observed that pinning of $a$ domains is associated with of pairs of misfit dislocations with Burgers vectors [100] and [001], as illustrated in the TEM results shown in Fig. 11(a-e). Model calculations shown in Fig. 11(f) of the in-plane strain field associated with the dislocation pair shown in Fig. 11(e) show the presence of a large region where the $a$ domain is favoured (red color), indicating that pinning of $a$ domains results primarily from the strong strain field produced by the dislocation pair. ${ }^{145}$

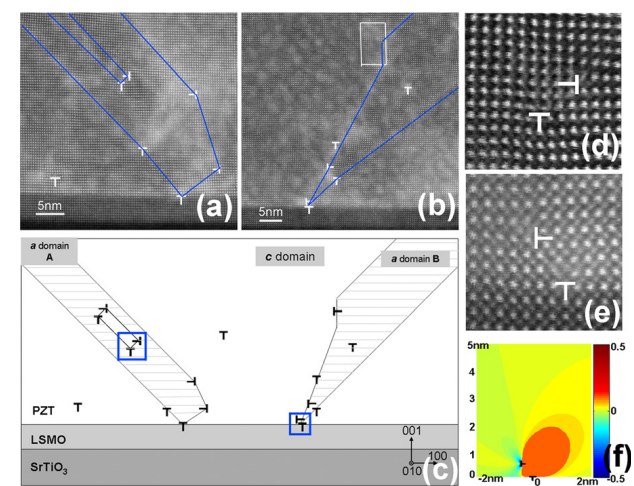

FIG. 11. Origin of domain wall pinning. (a) and (b) STEM ADF images along the [010] zone axis of two $a$ domains (marked by the solid lines) observed in the PZT(001) film, as schematically shown in (c). (d) and (e) STEM ADF images taken at higher magnifications on the areas marked in (c). (f) Calculated spatial variation of the elastic field $\epsilon_{\text {dis }, x x}$, with the interface between PZT and substrate set at $y=0$; the region where $\epsilon_{\mathrm{dis}, x x}>0.04$ (red) is the area where the $a$ domain should be stable. Reproduced from Appl. Phys. Lett. 99, 102902 (2011), with the permission of AIP Publishing. 
Epitaxial ferroelectric interfacial devices

One growth strategy for minimising the nucleation of a polydomain state has been to employ stepped substrate surfaces obtained from cutting the wafer off the main index plane by up to a few degrees (miscut or vicinal surfaces). The role of the stepped surface is that of allowing an asymmetrical strain relaxation along the direction of the steps, ${ }^{546,547}$ setting a preferential crystallographic direction for strain relaxation and for the growth of systems with lower symmetry than that of the substrate. Such an approach has been used to reduce the number of domain variants of $\mathrm{BiFeO}_{3}$ films grown on $\mathrm{SrTiO}_{3}(001)$ from four to two, resulting in an improvement in the ferroelectric switching characteristics of the $\mathrm{BiFeO}_{3}$ film, ${ }^{548,549}$ or to tune the crystal structure of $\mathrm{Pb}\left(\mathrm{Mg}_{1 / 3} \mathrm{Nb}_{2 / 3}\right) \mathrm{O}_{3}-\mathrm{PbTiO}_{3}$ thin films. ${ }^{550,551}$ The steps tend also to act as nucleation sites for film growth and can lead to a step flow growth mode and to atomically smooth surfaces; ${ }^{552}$ however, the regions where the steps meet are sites susceptible to the formation of lattice defects, such as strain induced by residual lattice matching between the film and substrate, ${ }^{553}$ stacking faults, ${ }^{551}$ and vertical lattice mismatch as a result of step bunching; ${ }^{554}$ additionally, the atomic steps interact with dislocations and modify their configuration in the film. ${ }^{555}$

Defect chemistry also plays an important role at the growth temperature. Intrinsic defects, such as vacant sites (vacancies), atoms occupying interstitial sites (interstitials), Frenkel defects (atoms that move to occupy an interstitial site leaving behind a vacancy), and Schottky defects (paired anion and cation vacancies) are thermodynamically favoured and will be always present in the crystal, since they reduce the Gibbs free energy of the system by increasing the entropy. ${ }^{556}$ In the oxide perovskites, double ionized oxygen vacancies are one of the most important type of point defects; ${ }^{557}$ their equilibrium density at a given temperature $T$ and oxygen partial pressure $p\left[\mathrm{O}_{2}\right]$ can be obtained from the chemical reaction, ${ }^{409,557-560}$

$$
\mathrm{O}_{\mathrm{O}}^{\times} \rightleftharpoons \frac{1}{2} \mathrm{O}_{2}(\mathrm{~g})+\mathrm{V}_{\mathrm{O}} \ddot{+}+2 \mathrm{e}^{\prime},
$$

in the Kröger-Vink notation, ${ }^{561}$ where $\mathrm{O}_{\mathrm{O}}^{\times}$is the lattice oxygen, $\mathrm{V}_{\mathrm{O}}$ the oxygen vacancy, and $\mathrm{e}^{\prime}$ the electron carrier density, describing the release (capture) of a pair of electrons to the conduction band of $\mathrm{SrTiO}_{3}$ for each oxygen vacancy created (annihilated). The corresponding reaction equilibrium constant $K_{1}$ is given by

$$
K_{1}=\frac{p\left[\mathrm{O}_{2}\right]^{1 / 2}\left[\mathrm{~V}_{\mathrm{O}}^{* \bullet}\right]\left[\mathrm{e}^{\prime}\right]^{2}}{\left[\mathrm{O}_{\mathrm{O}}^{\times}\right]}=e^{\Delta S / k_{\mathrm{B}}} e^{-H_{a} / k_{\mathrm{B}} T}
$$

where $\left[\mathrm{O}_{\mathrm{O}}^{\times}\right] \approx N_{0}$ is approximately constant and close to the number density of oxygen 
Epitaxial ferroelectric interfacial devices

sites $\left(N_{0}\right), p\left[\mathrm{O}_{2}\right]$ the oxygen partial pressure, $H_{a}$ and $\Delta S$ are the enthalpy (activation energy) and entropy of reaction, respectively, and $k_{\mathrm{B}}$ is the Boltzmann constant. For $\mathrm{SrTiO}_{3}$, $H_{a}=5.69 \mathrm{eV} /$ vacancy, ${ }^{558}$ for $\mathrm{BaTiO}_{3}, H_{a}=5.96 \mathrm{eV} /$ vacancy. ${ }^{562}$ One consequence of the presence of oxygen vacancies is that they contribute with charge carriers to the electrical conductivity, $\sigma_{i}=\mu_{i} n_{i} e$ (where $\mu_{i}$ is the charge mobility, $e$ the elementary electric charge, and $n_{i}$ the charge carrier density, where $i$ stands for electrons or hole carriers), with the expression above predicting a variation of $\left[\mathrm{e}^{\prime}\right]=2\left[\mathrm{~V}_{\ddot{\mathrm{O}}}\right] \propto p\left[\mathrm{O}_{2}\right]^{-1 / 6}$. Such variation is in fact observed, for example for $\mathrm{SrTiO}_{3}$ and $\mathrm{BaTiO}_{3}$, but only in the regime of high temperatures and strongly reducing conditions. ${ }^{409,557,558,562-564}$ More significant in determining the equilibrium concentration of oxygen vacancies is the effect of impurities (extrinsic point defects, typically present in ppm concentrations), which originate mostly from the source material and substitute for the intended atoms or occupy interstitial positions in the lattice (the latter being not favourable in closed packed lattices, such as the perovskite). Most impurities tend to be low valence cations, such as $\mathrm{Na}, \mathrm{Mg}$, and $\mathrm{Al}$, which act as acceptors when they replace cations with higher valencies (and as donors in the opposite case). ${ }^{409,557,565,566}$ Since they tend to be the dominant factor determining the oxygen vacancy concentration, in the reaction equation (5), $\mathrm{V}_{\mathrm{O}}$ is approximately constant and the electron carrier density now varies as $p\left[\mathrm{O}_{2}\right]^{-1 / 4}$, which is observed experimentally over wide temperature and oxygen pressure ranges. ${ }^{409,557,558,562-564}$ Under oxidizing conditions, excess oxygen fills in part the impurity related vacancies, leading to the introduction of pairs of hole carriers to the valence band (and $p$-type conductivity) leading to a dependence in hole carrier density $p \propto p\left[\mathrm{O}_{2}\right]^{+1 / 4}{ }^{567}$ For example, substitutional impurities such as $\mathrm{Na}, \mathrm{Mg}$, and $\mathrm{Al}$ act as acceptors and make PZT a $p$-doped ferroelectric (although the much lower mobility of minority electrons still makes the latter responsible for charge transport). ${ }^{177,409-411}$ In the intermediate regime, compensation of electron and hole charge carriers is reached and charge excitation becomes again intrinsic, i.e., determined by excitations over the band gap..$^{57,568-570}$ In particular, by deliberately doping the ferroelectric system with donors, the oxygen vacancy density can be reduced or fully compensated, resulting in softer ferroelectric properties. ${ }^{557,568,571}$ Systems with volatile elements, such as $\mathrm{Pb}$ in $\mathrm{PbTiO}_{3}$, or $\mathrm{Bi}$ in $\mathrm{BiFeO}_{3}$, may also have a significant density of cation vacancies at the growth temperature, ${ }^{409,572,573}$ while in systems where the electrons can be captured by multivalent cations, such as $\mathrm{Fe}$ in $\mathrm{BiFeO}_{3}$, the defect chemistry reaction is significantly different and more complex. ${ }^{571,574,575}$ In PZT, oxygen is released as PbO, such 
Epitaxial ferroelectric interfacial devices

that formation of oxygen vacancies is accompanied by the formation of $\mathrm{Pb}$ vacancies. ${ }^{409,576}$

While oxygen vacancies do not always have a negative impact on the properties of oxide materials (controlling the density of oxygen vacancies may in fact provide another knob to controlling their electronic properties ${ }^{577}$ and they are desirable for oxygen diffusion and ionic conductivity in solid electrolytes, such as in stabilized zirconia, YSZ ${ }^{578-581}$ ), they generally have a deleterious impact on the dielectric properties of ferroelectric oxides. ${ }^{409}$ They can contribute to the electrical conductivity (in $\mathrm{SrTiO}_{3}$ they form an impurity state in the band gap, just below the conduction band $)^{573,582}$ and modify the oxidation state of neighboring cations. Furthermore, oxygen vacancies are mobile, with an activation energy of the order of $0.6-1 \mathrm{eV}$ for the perovskite titanates, ${ }^{583-585}$ and can be displaced under the action of an applied electric field, or migrate at high temperature to the interface to screen the ferroelectric polarization, ${ }^{586}$ where the subsequent pile-up at one electrode interface and depletion at the other can contribute to resistance degradation, imprint effects, and fatigue in ferroelectric capacitor structures. ${ }^{583,587}$ It has been inferred that the high density of free carriers in thin PZT ferroelectric layers may be a consequence of the formation of oxygen vacancies induced at the temperature of growth in order to screen the surface ferroelectric polarisation (self-doping). ${ }^{412}$ Interfacial layers, by either acting as a sink, source, or by blocking the flow of oxygen, can affect the equilibrium oxygen vacancy density in the ferroelectric layer and impact its properties; ${ }^{409,572}$ in particular, by judiciously choosing the interfacial contact layers, improved retention and switching characteristics can be obtained. For example, the use of oxide electrodes in ferroelectric capacitors has been shown to reduce fatigue and imprint effects as compared with metal electrodes, which act to trap the oxygen vacancies within the ferroelectric. ${ }^{409}$

Since an oxygen vacancy corresponds to a missing oxygen anion, for example in the $\mathrm{TiO}_{6}$ octahedron of $\mathrm{BaTiO}_{3}$ or $\mathrm{Pb}\left(\mathrm{Zr}_{0.2} \mathrm{Ti}_{0.8}\right) \mathrm{O}_{3}$, oxygen vacancies also give rise to local electric dipoles that point along, against, or perpendicular to the local ferroelectric polarization and which strongly perturb the local electric field, affecting the ferroelectric switching properties (by acting as pinning centers for domain walls, for example) and contributing to imprint effects. ${ }^{571,575,583}$ For similar reasons, they also cause local distortions in the atomic lattice, resulting in local strains. The impact of the latter will depend on the particular system: one may expect it to be strong in displacive ferroelectrics, such as $\mathrm{BaTiO}_{3}$, while in lone-pair ferroelectric rhombohedral $\mathrm{BiFeO}_{3}$ the effect of oxygen vacancies (and of strain) has been 
Epitaxial ferroelectric interfacial devices

estimated to be minor. ${ }^{437}$

\section{FERROELECTRIC FIELD EFFECT AND CONTROL OF CORRELATED STATE}

Controlling the correlated state of matter is a fundamental topic in condensed matter physics, both because understanding the underlying mechanisms brings insights into the role of electron correlations to the emergence of novel physical phenomena and because those effects can potentially be useful for device applications. In fact, a major difficulty stems from the challenge of accounting fully for electron-electron many body interactions. ${ }^{588,589}$ Here we focus mostly on transition metal oxides, which are a prototypical class of systems exhibiting strong electron-electron correlations; this is manifested by the strong deviations in their behavior from that expected from a near-free electron system. Typical of these systems are complex phase diagrams, with several equilibrium states as a function of a given physical parameter, such as pressure or doping, as illustrated in the diagram shown in Fig. 12 representative of a wide class of $3 \mathrm{~d}$ transition metal oxides. Starting from the insulating parent oxide, the addition of charge carriers does not initially modify strongly the electronic state, but at a critical carrier density, the carriers become delocalized, leading to a new electronic conducting ground state; with further increase of the charge carrier density, electron-electron correlations lead to the opening of a band gap in the hybridized oxygen 2 p-transition metal $3 \mathrm{~d}$ band at half filling, resulting in a typical dome shape in the phase diagram. ${ }^{3}$ In fact, the general physical properties of strongly correlated systems may be described grosso modo in terms of the charge carrier density and electron bandwidth contributions of the Hubbard Hamiltonian. ${ }^{590}$ The electronic bandwidth is determined by the cationic crystal field and orbital overlap, while the charge carrier density can be modified through either chemical, ionic, or electrostatic doping. A classic example of the changes in the electronic properties of complex oxides with doping is that of the doped manganites $A \mathrm{MnO}_{3}$, where the average Mn valency can be modulated by varying the amount of di- or trivalent cations occupying the $A$ sites of the perovskite structure via chemical substitution. ${ }^{591-595}$ One drawback of this approach to modulating the charge carrier density is that it is irreversible and involves local changes in the crystal structure induced by the different atomic size of the substituting element. ${ }^{596}$ Another approach to modulating the charge carrier is through ionic doping, ${ }^{597-599}$ 
Epitaxial ferroelectric interfacial devices

based on ionic mass transport into or out of the material, for example oxygen or lithium, both of which have relatively high mobility in oxide materials ${ }^{559,560,600,601}$ and which can be driven by electric fields $\left(\mathrm{Li}^{+}\right.$or charged oxygen vacancies, $\left.\mathrm{V}_{\mathrm{O}}^{2+}\right)$. While in general a change in the oxygen content in an oxide material is undesirable (an exception being in ionic conductors, such as stabilised zirconia, as mentioned earlier), it has been used to advantage for example in memristor devices, where different resistive states can be obtained by drastic modifications in the oxygen atom distribution under electric breakdown conditions, ${ }^{602}$ or to control the interfacial oxidation and the interfacial magnetic anisotropy of $\mathrm{Co} / \mathrm{GdO}_{x}$ by an applied electric field. ${ }^{598}$ Here, we focus on electrostatic doping, which relies on charge screening effects to modulate the charge carrier density at the interface between a dielectric and a conducting material, as in the classic field effect transistor. Unlike semiconductors, however, which are characterized by relatively low intrinsic carrier densities $\left(10^{10}-10^{18} \mathrm{~cm}^{-3}\right),{ }^{189}$ strongly correlated materials have very high charge carrier densities at the Fermi level, of the order of $10^{21} \mathrm{~cm}^{-3}$, requiring therefore very large induced surface charge polarizations at the dielectric interface which are not possible to achieve with conventional $\mathrm{SiO}_{x}$ gates. Instead, large reversible modulation of the carrier density can be achieved in field effect device structures using either high dielectric constant materials or ferroelectrics, whose spontaneous surface bound charge is used to induce large modulations in the charge carrier density at the interface in ferroelectric field effect device structures; $;, 8,10,603,604$ an alternative is to employ electrolytes, i.e., ionic conductors, where the electric field is screened at the electrode interface by mobile ions that leads to the formation of a so-called double-layer of electric charge within a nanometer of the interface and no electric field within the electrolyte. ${ }^{605,606}$

The original proposal for the ferroelectric field effect transistor ${ }^{58,59,607-609}$ aimed at combining the binary memory function of the ferroelectric polarization direction with the field effect control of the conductivity in a semiconductor channel in order to achieve a non-volatile transistor switch, i.e., to extend the classical function of a field effect transistor. An extension of this concept consists of replacing the semiconducting channel layer by a strongly-correlated material in order to control electrostatically its electronic state. ${ }^{4,5,85,610,611}$ The goal is to expand the functionality of such types of device structures by controlling the magnetic state, orbital or charge order, superconductivity, or the metal to insulator transition. A schematic of the ferroelectric field effect device structure and of the basic approach to controlling the electronic state of the channel layer is shown in Fig. 12: by choosing a chemical doping in 
Epitaxial ferroelectric interfacial devices

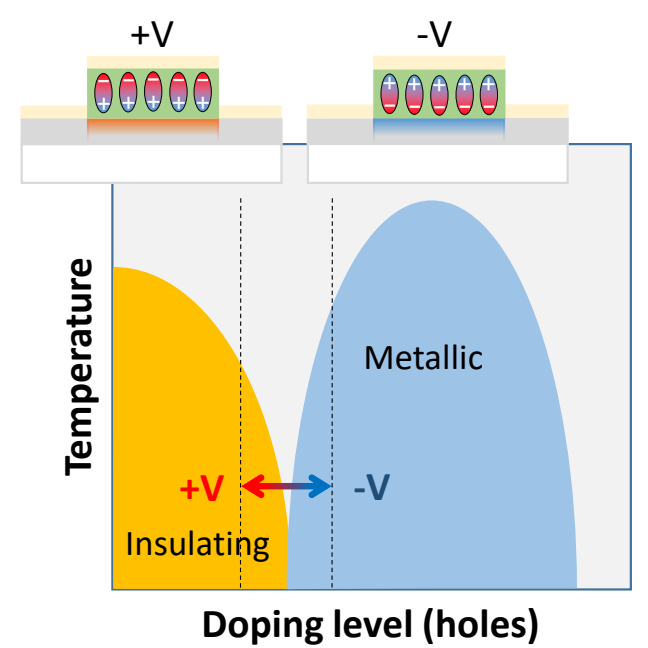

FIG. 12. Schematic representation of the operation of the ferroelectric field effect device used to drive the channel system across two equilibrium states in the temperature-charge density phase diagram characteristic of $3 \mathrm{~d}$ transition metal complex oxides.

the phase diagram of a strongly correlated system near the boundary between two different ground states, the system is driven across the boundary (horizontal arrow in the figure) through electrostatic carrier modulation, induced by charge carrier depletion or accumulation at the ferroelectric interface. ${ }^{3,317}$ In these device structures, the role of the ferroelectric layer is to provide the required change in the interfacial carrier density through its surface bound charge as the direction of the ferroelectric polarization is reversed by application of a voltage to the gate contact. The practical requisites have been discussed in section II and include, for optimal effects, a low density of defects; an abrupt interface with a low charge trap density and minimal interdiffusion; large ferroelectric polarizations; and Schottky contacts to enable application of electric fields for ferroelectric switching. Alternatively, high dielectric materials such as $\mathrm{SrTiO}_{3}$ can be employed as a gate dielectric, which are capable of providing large surface polarizations at relatively modest applied electric fields, although, in contrast to ferroelectrics, the effect will be present only as long as the electric field is applied to the system. Based on such device structures, control of several physical properties of correlated materials has been achieved and will be discussed in the following sections. These 
Epitaxial ferroelectric interfacial devices

include control of the superconducting state in the cuprates and of the Mott insulator to metal transition in Mott insulators, and of spin, charge and orbital order in several metal oxide systems.

\section{A. Control of the superconducting state}

Superconductivity is one classic example of the effect of electron-electron and electronphonon interactions inducing surprising physical phenomena in the solid state, such as zero resistivity and the expulsion of magnetic field lines known as the Meissner effect. In fact, while a good understanding of type $I$ superconductors has been achieved through the Bardeen-Cooper-Schrieffer (BCS) model, ${ }^{612,613}$ a complete physical understanding of type $I I$, high- $T_{\mathrm{c}}$, or unconventional superconductivity, occurring at temperatures where the BCS theory would preclude superconductivity, has still not been reached yet. ${ }^{614-616}$ Given the intimate link between superconductivity and charge carrier density, it is not surprising that control of the superconducting state through electrostatic doping was attempted early, with the first reports dating back to the 1960s demonstrating small modulations in the superconducting critical temperature in metals, of the order of $0.1-1 \mathrm{mK} .{ }^{617-619} \mathrm{In}$ addition to offering one knob for controlling the physics governing the electron pairing mechanism, and a perspective for a better understanding of superconducting phenomena by avoiding compositional structural modifications that come with chemical doping, ${ }^{3,620}$ electrostatic control of superconductivity is also of potential interest for device applications, such as superconducting FETs, where in principle, switching the system between the superconducting and normal states could result in very high on/off resistance ratios. ${ }^{621-625}$ From the BCS expression for the critical temperature, ${ }^{612,613,626,627}$

$$
k_{\mathrm{B}} T_{\mathrm{c}}=1.14 \hbar \omega e^{-1 / N_{0} V_{0}},
$$

where $k_{\mathrm{B}}$ is the Boltzmann constant, $\omega$ and $V_{0}$ are the parameters of the BCS effective interaction (with $\hbar \omega / k_{\mathrm{B}}$ of the order of the Debye temperature), and $N_{0}=g\left(E_{\mathrm{F}}\right) / 2$ is the (free electron) density of electronic levels for a single spin population in the normal metal, it follows that

$$
\frac{\Delta T_{\mathrm{c}}}{T_{\mathrm{c}}}=\frac{1}{N_{0} V_{0}} \frac{\Delta N_{0}}{N_{0}} \equiv \beta \frac{\Delta N_{0}}{N_{0}}
$$


Epitaxial ferroelectric interfacial devices

predicting a proportional increase (decrease) in the critical temperature with electron accumulation (depletion), consistent with earlier observations. ${ }^{617-619}$

In superconductors, in addition to the screening length over which electric fields can extend into the system, two other important characteristic length scales are the London penetration depth, $\lambda_{\mathrm{L}}$, over which magnetic fields and electric currents can be present in the superconductor, and the coherence length $\xi$, over which changes in the order parameter occur. The response to magnetic fields depends strongly on whether the London penetration depth is smaller or larger than the coherence length, $\xi$, separating superconductors into type I and II, respectively. ${ }^{627}$ For electric fields, the relevant comparison is between the ThomasFermi screening length and the coherence length perpendicular to the surface, $\xi_{\perp},{ }^{624,628}$ with stronger field effects expected when $\delta_{\mathrm{TF}} \geq \xi_{\perp}$. Since in metals the electron density is high, the Thomas-Fermi screening length and the change in the carrier density within $\xi_{\perp}$ are small, such that the changes in $T_{\mathrm{c}}$ are also correspondingly small. One way to optimize the field effect is to reduce the film thickness down to a minimum where superconductivity is still observed (of the order of $\xi_{\perp}$ ) and ideally to a value comparable to the ThomasFermi screening length. Using such an approach, a gradual transition from the normal to the superconducting state could be observed in a $10 \AA$ thick bismuth film grown on $\mathrm{SrTiO}_{3}(001)$ upon accumulation of charge carriers through electrostatic doping using the substrate as the gate dielectric. ${ }^{629}$ In this example it was argued that, in addition to the change in the carrier density, the electronic screening (described by $V_{0}$ ) was modulated by the increase in electron density. Reducing the lateral dimensions of the system can also result in larger and new field effects, as illustrated by recent field effect experiments carried out in $\mathrm{Al}$ and $\mathrm{Ti}$ planar wire structures fabricated on Si substrates, showing an ambipolar modulation of the critical current that is not expected from a conventional modulation in the electron density. ${ }^{625,630}$ The results mentioned thus far are for polycrystalline metallic films of type I superconductors, indicating that field effect control of the superconducting state is robust, even if both the size of the effect and the critical temperatures tend to be small in these systems.

To achieve larger perturbations in the superconducting state using field effects, systems with larger screening lengths and shorter perpendicular coherence lengths are the most suited. ${ }^{631,632}$ In this context, the discovery of high temperature superconductivity in the cuprates $^{633}$ made much larger field effect modulations of superconductivity possible. ${ }^{632}$ The 
Epitaxial ferroelectric interfacial devices

cuprates consist of electron- or hole-doped layered perovskites with phase diagrams similar to those schematically shown in Fig. 12, exhibiting an antiferromagnetic phase at low doping that competes with the superconducting phase at higher doping, with a critical temperature that peaks at the so-called optimal doping. Between the antiferromagnetic and superconducting region, a pseudo-gap phase emerges for hole doped systems. ${ }^{590,634,635}$ These systems offer several advantages for field effect modulation, including much lower carrier densities as compared to metallic superconductors, a much smaller coherence length along the crystalline $c$ direction (superconductivity being essentially two-dimensional, occurring in the $\mathrm{CuO}_{2}$ planes of the layered structure) and, importantly, much higher critical temperatures of up to $135 \mathrm{~K}$ at ambient pressure. ${ }^{636}$ In addition, the presence of boundaries in the doping phase diagram separating superconducting from normal and antiferromagnetic phases suggests that direct changes in the ground state of the system may be achieved by crossing one such boundary using electrostatic doping. ${ }^{632}$ Indeed, much larger modulations in superconductivity induced by field effects were reported for films of cuprate superconductors, first by using a gate dielectric to modulate the charge carrier density, ${ }^{620,637-645}$ and later by using ferroelectric gates, ${ }^{507,646-654}$ electrolytes, ${ }^{655,656}$ and also ionic doping. ${ }^{657}$ In Table V we list several examples of systems where the variation in $T_{\mathrm{c}}$ has been obtained through electrostatic doping, together with a figure of merit $\beta$ that gives a measure of the relative change in $T_{\mathrm{c}}$ for a relative change in carrier density via equation (8).

By using dielectric gates to electrostatically control the charge density, large modulations in the critical temperature and/or critical current of high $T_{\mathrm{c}}$ superconductors have been observed. Although the effect is non-persistent, this approach has provided interesting insights into the physics of high-temperature superconductors. For example, using a $7 \mu \mathrm{m}$ thick Kapton dielectric gate, Fiory et al. ${ }^{637}$ studied the variation of the effective mass and hole carrier density of $\mathrm{YBa}_{2} \mathrm{Cu}_{3} \mathrm{O}_{7}$ films, to show that the hole carrier density is constant in temperature (except in a region near $T_{\mathrm{c}}$ ) and to obtain an estimate of the mass of the Cooper pair, $m^{*}=(5.5 \pm 0.5) m_{e}$ at $T<70 \mathrm{~K} .{ }^{663,664}$ The first result showed that the hole carrier density cannot be estimated from the Hall coefficient directly, which instead exhibits a marked variation with temperature in the cuprates. ${ }^{507,657,660,665,666}$ Further, it suggested that the critical temperature of the surface layer (probed by modulating the excitation current) is the same as that of the bulk of the film (30 nm thick). Larger effects could be reached using high dielectric constant oxides, including $\mathrm{SrTiO}_{3}$ and $\mathrm{Ba}_{x} \mathrm{Sr}_{1-x} \mathrm{TiO}_{3}$, with changes in $T_{\mathrm{c}}$ of 
Epitaxial ferroelectric interfacial devices

TABLE V. Electrostatic control of the superconducting critical temperature (ordered chronologically and by type of gate, dielectric or ferroelectric). *Thickness $(t)$ converted to unit cells (u.c.) using the lattice parameter of $\mathrm{YBaCu}_{3} \mathrm{O}_{x}, c=11.68 \AA .{ }^{658} T_{\mathrm{c}}$ is the critical temperature, $\Delta T_{\mathrm{c}}$ the change in $T_{\mathrm{c}}$ for the change in surface charge density $\Delta Q$, and $n$ is the average carrier density (values in parenthesis are estimated from the expected number of hole carriers per $\mathrm{CuO}_{2}$ unit). IST: insulator to superconductor transition. †Deduced from films grown on the two opposite faces of the $\mathrm{LiNbO}_{3}$ crystal. $\ddagger$ Estimated from Ref. 659 .

\begin{tabular}{|c|c|c|c|c|c|c|c|}
\hline System & $\begin{array}{l}t \\
\text { (u.c.) }\end{array}$ & $\begin{array}{l}T_{\mathrm{c}} \\
(\mathrm{K})\end{array}$ & $\begin{array}{l}\Delta T_{\mathrm{c}} \\
(\mathrm{K})\end{array}$ & $\begin{array}{l}n \\
\left(\times 10^{21} \mathrm{~cm}^{-3}\right)\end{array}$ & $\begin{array}{l}\Delta Q \\
\left(\mu \mathrm{C} / \mathrm{cm}^{2}\right)\end{array}$ & $\beta$ & Ref. \\
\hline Kapton $/ \mathrm{YBa}_{2} \mathrm{Cu}_{3} \mathrm{O}_{7}$ & 30 & 89.9 & $+1.1 \times 10^{-4}$ & 4.1 & 0.13 & 0.007 & 637 \\
\hline $\mathrm{STO} / \mathrm{YBa}_{2} \mathrm{Cu}_{3} \mathrm{O}_{7-x}$ & $2.3^{*}$ & 8 & -2 & 5 & -40 & 1 & 640 \\
\hline $\mathrm{STO} / \mathrm{YBa}_{2} \mathrm{Cu}_{3} \mathrm{O}_{7-x}$ & $4.7^{*}$ & 20 & -2 & 5 & -40 & 1 & 640 \\
\hline $\mathrm{BTO} / \mathrm{Sm}_{0.7} \mathrm{Ca}_{0.3} \mathrm{Ba}_{2} \mathrm{Cu}_{3} \mathrm{O}_{7-x}$ & 50 & 40 & +1 & $0.6 \ddagger$ & 8.0 & 1.5 & 641 \\
\hline $\mathrm{Bi}_{2} \mathrm{Sr}_{2} \mathrm{CaCu}_{2} \mathrm{O}_{8+x} / \mathrm{BSTO}$ & 21 & 24 & -2 & 1.8 & -25 & 2 & 642 \\
\hline $\mathrm{YBa}_{2} \mathrm{Cu}_{3} \mathrm{O}_{7-x} / \mathrm{BSTO}$ & 10 & 68 & -1 & 2.3 & -25 & 0.2 & 642 \\
\hline $\mathrm{STO} / \mathrm{PrBa}_{2} \mathrm{Cu}_{3} \mathrm{O}_{7-x} / \mathrm{STO}$ & $2.3^{*}$ & 17.4 & +2.8 & $(2.3)$ & 4 & 3 & 644 \\
\hline $\mathrm{Al}_{2} \mathrm{O}_{3} / \mathrm{Nd}_{1.2} \mathrm{Ba}_{1.8} \mathrm{Cu}_{3} \mathrm{O}_{y}$ & $9.3^{*}$ & 18 & IST & 0.56 & 2 & - & 620,645 \\
\hline $\mathrm{YBa}_{2} \mathrm{Cu}_{3} \mathrm{O}_{7-x} / \mathrm{BTO}$ & 100 & 83 & 0.5 & 4 & 14 & 3 & 646,647 \\
\hline $\mathrm{YBa}_{2} \mathrm{Cu}_{3} \mathrm{O}_{7-x} / \mathrm{LNO} \dagger$ & 100 & 60 & 40 & 4 & 140 & 30 & 646,647 \\
\hline $\mathrm{PZT} / \mathrm{GdBa}_{2} \mathrm{Cu}_{3} \mathrm{O}_{7-x}$ & 2 & 50 & 7 & 2 & 20 & 0.4 & 649 \\
\hline $\mathrm{PZT} / \mathrm{STO} / \mathrm{GdBa}_{2} \mathrm{Cu}_{3} \mathrm{O}_{7-x}$ & 8.8 & 35.2 & 0.65 & 4 & 46 & 0.2 & 650 \\
\hline $\mathrm{PZT} / \mathrm{NdBa}_{2} \mathrm{Cu}_{3} \mathrm{O}_{7-x}$ & 10 & 60 & 1 & 1.25 & 20 & 0.2 & 651 \\
\hline $\mathrm{PZT} / \mathrm{NdBa}_{2} \mathrm{Cu}_{3} \mathrm{O}_{7-x}$ & 8 & 78.4 & 1 & 1.5 & 20 & 0.1 & 652 \\
\hline $\mathrm{BFO} / \mathrm{YBa}_{2} \mathrm{Cu}_{3} \mathrm{O}_{7-x}$ & $3.5^{*}$ & 40 & 30 & 0.9 & 130 & 0.3 & 653 \\
\hline $\mathrm{BFO} / \mathrm{YBa}_{2} \mathrm{Cu}_{3} \mathrm{O}_{7-x}$ & $4.7^{*}$ & 40 & 15 & 0.88 & 130 & 0.2 & 654 \\
\hline $\mathrm{BFO} / \mathrm{YBa}_{2} \mathrm{Cu}_{3} \mathrm{O}_{7-x}$ & $3.5^{*}$ & $(55)$ & 28 & $(0.9)$ & 140 & 0.2 & 660 \\
\hline $\mathrm{BFO} / \mathrm{YBa}_{2} \mathrm{Cu}_{3} \mathrm{O}_{7-x}$ & $4.7^{*}$ & 38 & 8 & 1 & 120 & 0.1 & 507 \\
\hline $\mathrm{PZT} / \mathrm{Sr}\left(\mathrm{Ti}_{0.98} \mathrm{Nb}_{0.02}\right) \mathrm{O}_{3}$ & 26 & 0.266 & 0.06 & 0.05 & 12 & 0.4 & 661,662 \\
\hline
\end{tabular}

a few K. Since the oxygen stoichiometry determines hole doping and $T_{c}$ in $\mathrm{YBa}_{2} \mathrm{Cu}_{3} \mathrm{O}_{7-x}$, 
Epitaxial ferroelectric interfacial devices

an important aspect is whether mobile oxygen vacancies are at the origin of the electric field modulation of superconductivity, a question that was answered in the negative in the study by Frey et $a l^{642}$ by showing no significant differences in the field effect response between $\mathrm{YBa}_{2} \mathrm{Cu}_{3} \mathrm{O}_{7-x}$ and a well oxidized superconductor $\mathrm{Bi}_{2} \mathrm{Sr}_{2} \mathrm{CaCu}_{2} \mathrm{O}_{8+x}$, ruling out a major contribution from electric field induced oxygen diffusion. ${ }^{643}$ Dielectric gates have also been employed to control the onset of superconductivity induced by field effects at the $\mathrm{LaAlO}_{3} / \mathrm{SrTiO}_{3}$ interface. ${ }^{667-673}$ In this system, the carrier density could be modulated to map a large region of the temperature-doping space parameter, which exhibits a domelike shape superconducting state within the normal state and indicating the presence of a quantum critical point. ${ }^{667}$ Rather than a result of a simple change in the sheet carrier density, the main impact has been ascribed to changes in the electron charge mobility as a result of compression or expansion of the electron gas due to band bending. ${ }^{674,675}$ An alternative approach to probing the phase space electrostatically is by ionic doping, such as in $\mathrm{Bi}_{2} \mathrm{Sr}_{2} \mathrm{CaCu}_{2} \mathrm{O}_{8+x},{ }^{657}$ achieved by growing the film on a lime-glass substrate and by changing the carrier density through the diffusion of $\mathrm{Na}^{+}$cations induced by the applied electric field. Here also, the effect of lattice distortions that arise with chemical doping is avoided.

Larger and non-volatile modulations in $T_{\mathrm{c}}$ are found in ferroelectric gated cuprate superconductors, of up to $30 \mathrm{~K}$ in $\mathrm{YBa}_{2} \mathrm{Cu}_{3} \mathrm{O}_{7-x},{ }^{653}$ and where, in some instances, switching between the normal metallic and superconducting state could be achieved. ${ }^{620,645}$ One strategy to minimize the film thickness has been to employ a suitable buffer layer, such as $\mathrm{PrBa}_{2} \mathrm{Cu}_{3} \mathrm{O}_{7},{ }^{649,654}$ a semiconductor that acts to decouple the superconductor from the $\mathrm{SrTiO}_{3}$ substrate and adds a first layer of $\mathrm{CuO}$ chains and metallicity that are thought to promote superconductivity in the superconducting layer. ${ }^{676,677}$ An example is provided in Fig. 13 for $\mathrm{BiFeO}_{3} / 3$ u.c. $\mathrm{YBa}_{2} \mathrm{Cu}_{3} \mathrm{O}_{7-x}$, showing that the critical temperature can be shifted by $30 \mathrm{~K}$ between the two polarization states of $\mathrm{BiFeO}_{3} \cdot{ }^{653}$ This example also shows that the change in $T_{\mathrm{c}}$ decreases with increasing film thickness, as expected for a field effect and for the screening length of $\mathrm{YBa}_{2} \mathrm{Cu}_{3} \mathrm{O}_{7-x}$, of about $1 \mathrm{~nm} .{ }^{624}$ One other interesting prospect for ferroelectric field effect devices ensues from the possibility of locally controlling the ferroelectric domain pattern ${ }^{678,679}$ to create nanoscale superconducting devices, for example, to create weak links in device structures. ${ }^{660}$ Examples of other systems where ferrelectric control of the superconducting state has been reported include $\mathrm{Nb}$-doped $\mathrm{SrTiO}_{3}$, where 
Epitaxial ferroelectric interfacial devices

on/off switching of superconductivity has been demonstrated using PZT as a ferroelectric gate. ${ }^{661,662}$
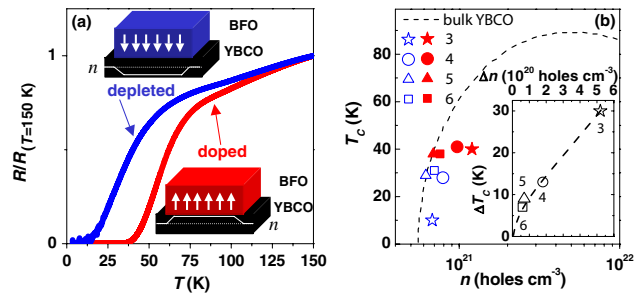

FIG. 13. (a) Temperature variation of the resistance of a $\mathrm{BiFeO}_{3} / 3$ u.c. $\mathrm{YBa}_{2} \mathrm{Cu}_{3} \mathrm{O}_{7-x}$ (BFO/YBCO) heterostructure (normalized to the resistance at $150 \mathrm{~K}$ and measured at $J=1.7$ $\mathrm{kA} / \mathrm{cm}^{2}$ ) for two neighboring areas in which the $\mathrm{BiFeO}_{3}$ polarization points along opposite directions, as shown in the figure insets. (b) Superconducting critical temperature $T_{\mathrm{c}}$ vs charge carrier density $n$ for samples with different $\mathrm{YBa}_{2} \mathrm{Cu}_{3} \mathrm{O}_{7-x}$ thicknesses (in u.c.), for the depletion (empty symbols) and accumulation (solid symbols) states. Inset: variation of $T_{\mathrm{c}}$ with $n$. Reproduced with permission from Phys. Rev. Lett. 107, 247002 (2011). Copyright 2011 by the American Physical Society.

\section{B. Control of the Mott insulator to metal transition}

The metal-insulator transition refers to a change in the conducting state of the system, between metallic and insulating regimes, driven by a control parameter, such as temperature, pressure, charge doping, magnetic field, or disorder. ${ }^{590,680-685}$ Several physical mechanisms can be responsible for such a transition, including changes in band overlap (Wilson metal-insulator transition), disorder (Anderson metal-insulator transition), charge instabilities (Peierls metal-insulator transition) and electron correlations (Mott metal-insulator transition) ${ }^{686}$ Although we focus here on electric field control of the correlated state, we discuss briefly for completeness the other metal-insulator transition mechanisms. The physics underlying metal-insulator transitions is complex and touches on several fundamental concepts in solid state physics, including the role of electron correlations, disorder, electronic band structure, and underlies important magnetic and transport phenomena such as superconductivity and colossal magnetoresistence. ${ }^{590}$ 
Epitaxial ferroelectric interfacial devices

One point of interest in using the metal-insulator transition for logic switches is that of achieving smaller subthreshold swings, $S=d V_{g} / d\left(\log I_{d}\right)$ (gate voltage $V_{g}$ needed to reduce the drain current $I_{d}$ by one decade), to achieve lower switching energies than is currently possible with MOSFETs, where $S \approx\left(1+C_{d} / C_{i}\right)\left(k_{\mathrm{B}} T / q\right) \ln 10$ is essentially limited by the thermal excitation of the charge carriers to $\left(k_{\mathrm{B}} T / q\right) \ln 10 \approx 60 \mathrm{mV} / \mathrm{dec}$ at room temperature $\left(C_{d}\right.$ and $C_{i}$ are the capacitance of the depletion and oxide layers, respectively, $T$ the temperature and $q$ the elementary charge value). ${ }^{189,687}$ Hence, one motivation for Mott FET transistors is that by replacing the physical mechanism driving the charge carrier density limited by Boltzmann statistics to a faster and more abrupt process based on a phase transition, one may achieve smaller subthreshold swings. ${ }^{687}$ In this context, it is worth mentioning a new device concept based on "negative capacitance" seeks to reduce the $m=$ $1+C_{d} / C_{i}$ factor by designing the gate structure such that an effective negative value for $C_{d}$ is obtained. ${ }^{688-690}$

\section{Wilson metal-insulator transition}

One mechanism for the onset of the metal-insulator transition is due to band overlap (Wilson metal-insulator transition), such as in divalent metals where metallicity is due to overlap between the first and second zones and where an insulating state is expect to set in when the atomic distance is increased to the point where reduced orbital overlap leads to electron localization and conduction becomes possible only through hopping. ${ }^{683,691}$ Transitions of this kind occur in several materials under high pressure, where the reduced lattice parameter (larger orbital overlap) leads to band overlap and to the onset of an insulator to metal transition, such as in iodine, ${ }^{692-695}$ selenium, ${ }^{693,694}$ and oxygen. ${ }^{696-699}$ A more familiar example of (electrostatic) control of band overlap in band insulators is in semiconductors, such as in the MOSFET transistor, where band bending under a gate voltage changes the channel layer from a back-to-back diode junction to a conducting inversion layer, where electron confinement gives rise to a two-dimensional electron gas. ${ }^{189,700}$ Another example of control of the conduction state in band insulators is that of the $\mathrm{LaAlO}_{3} / \mathrm{SrTiO}_{3}(001)$ system, where at a critical $\mathrm{LaAlO}_{3}$ thickness of about 4 u.c. the system transitions from insulator to metallic by the onset of a $2 \mathrm{D}$ electron gas state that sets in at the interface ${ }^{701}$ (which can be further driven into a superconducting state by gating). ${ }^{667-673}$ (Various mechanisms 
Epitaxial ferroelectric interfacial devices

have been advanced for explaining the onset of the $2 \mathrm{D}$ electron gas state at the interface, including band bending, polar catastrophe, interfacial strain effects, and oxygen vacancies. $)^{702}$ Field effect devices employing $\mathrm{SrTiO}_{3}{ }^{687,703-707}$ and $\mathrm{KTaO}_{3}{ }^{708,709}$ as the channel layer have also been shown to display large changes in resistivity as a function of applied gate voltage, with on/off current ratios of up to $10^{5}$. In one instance, a transition from metallic to insulator state in $n$-doped $\mathrm{SrTiO}_{3}$ with applied gate voltage has been observed, where a $\mathrm{CaHfO}_{3}$ layer is used as the gate insulator (epitaxial at the interface but amorphous above 4 unit cells thickness). ${ }^{706}$ This effect is observed as a change in the temperature variation of the channel conductance, from a resistive to a conductive regime, at a bias voltage of $1.3 \mathrm{~V}$; the conduction mechanism is explained in terms of thermal carrier excitation with an activation energy barrier that is found to collapse (i.e., drop to zero) for bias voltages above $1.3 \mathrm{~V}$, leading to a metallic state (the conductivity in the insulating state being attributed to the presence of in-gap states). Interestingly, from a scaling analysis of the resistivity data, it was concluded that the system behaves as three dimensional.

Another example that could be described as a Wilson insulator to metal transition is found to occur at the PZT/ $\mathrm{LaNiO}_{3}$ interface, where changes in the conductivity of $\mathrm{LaNiO}_{3}$, a metallic oxide, are found to be much larger than expected based on the charge carrier modulation in the thin $\mathrm{LaNiO}_{3}$ channel layer (3-4 unit cells). ${ }^{710}$ The results of ab initio calculations, shown in Fig. 14, suggest that the PbO interface layer of PZT in contact with the $\mathrm{TiO}_{2}$ layer of $\mathrm{LaNiO}_{3}$ is subject to an upward band shift when going from the depletion to the accumulation state that makes it cross the Fermi level, leading to the appearance of a conductive $\mathrm{PbO}$ state and to a new conducting channel at the interface.

\section{Anderson metal-insulator transition}

The presence of disorder, such as atomic disorder, can also lead to the onset of an insulating state (Anderson metal-insulator transition), whereby deviations from the periodic lattice potential result in localization of the electronic states near the Fermi energy and to charge transport through hopping between states separated by varying energy barriers. ${ }^{680,711}$ In weakly disordered Anderson insulators, a metallic state can be induced by increasing the charge density such that the Fermi energy rises to above the localized states to reach the metallic states (at the so-called mobility edge) ${ }^{681,711,712}$ by electrostatic doping, for example, 
Epitaxial ferroelectric interfacial devices
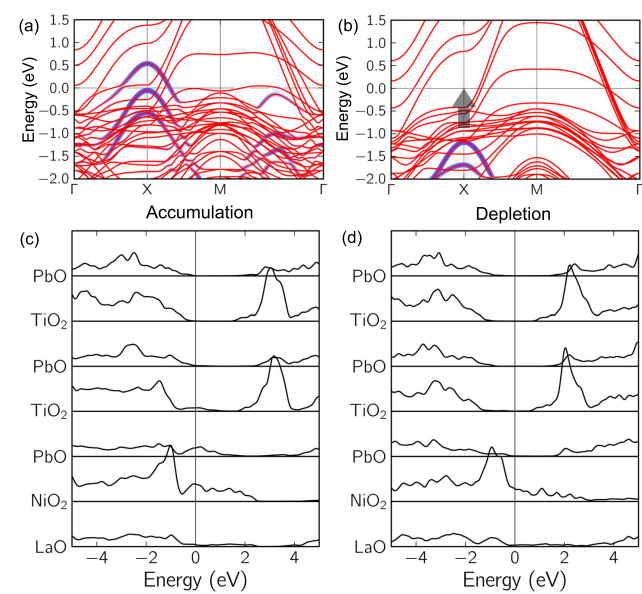

FIG. 14. Band structure of $\mathrm{PTO} / \mathrm{LaNiO}_{3}$ strained to the theoretical in-plane lattice constant of $\mathrm{LaAlO}_{3}$ for (a) accumulation and (b) depletion, where the zero of energy is the Fermi level in each case and a $(1 \times 1)$ interfacial unit cell is employed. Red-colored bands correspond to dominant $\mathrm{LaNiO}_{3}$ character, while blue-colored bands indicate strong contributions from the interfacial $\mathrm{PbO}$ layer. The top of the PbO-dominated bands shift from about $-1.2 \mathrm{eV}$ in depletion to approximately $0.5 \mathrm{eV}$ in accumulation. (c) The layer-resolved LDOS at the interface for accumulation and (d) depletion. The $\mathrm{PbTiO}_{3}$ is insulating in depletion and in accumulation away from the interface, but exhibiting a finite LDOS at the Fermi level at the interface in the latter case. Reproduced with permission from Phys. Rev. Applied 2, 051001 (2014). Copyright 2014 by the American Physical Society.

a process that has been extensively investigated in disordered semiconductors. ${ }^{711}$ Disorderinduced localization effects can also play an important role in the transport properties of correlated oxides, for example due to the short-range disorder associated with the atomic substitution in mixed valent materials, such as La and $\mathrm{Sr}$ site disorder in $\mathrm{La}_{0.8} \mathrm{Sr}_{0.2} \mathrm{MnO}_{3}$, which has been associated to the onset of a disorder-induced insulating state in thin films at low temperatures. ${ }^{713}$ 
Epitaxial ferroelectric interfacial devices

\section{Peierls metal-insulator transition}

Charge instabilities induced by electron-phonon interactions in low dimensional systems can lead to changes in the atomic and band structure and also result in a transition from a conducting to an insulating state (Peierls metal-insulator transition). ${ }^{687,714,715}$ Peierls insulators are typically strongly anisotropic materials characterized by exotic charge transport properties, in that charge can be transported by the charge density wave above an applied voltage threshold. ${ }^{715}$ To our knowledge, electrostatic control of the Peierls metal-insulator transition has not been reported in the literature.

\section{Mott metal-insulator transition}

In contrast to early expectations of electron band theory, many systems containing an odd number of charge carriers per atom are insulators, such as $\mathrm{NiO}$, a behaviour that was explained in terms of the role played by electron correlations (electron-electron Coulomb interaction), which favors electron localization (singly occupied electron states) and a gap opening in an otherwise half-filled electron band, resulting an insulating state (Mott insulators). ${ }^{590,683}$ In fact, until recently it was thought that the failure of spin dependent local density functional theory (DFT) to correctly predict the opening of band gaps in many complex materials was associated with the shortcomings in accounting for the electron correlation energy in a mean field approximation; however, recent results indicate that the opening of band gaps in DFT ensues once the early constraints imposed to the simulated system (such as cell size, atomic relaxation, breaks in symmetry) are relaxed, indicating that the exchange functionals used are sufficient to describe the band structure of complex oxides. ${ }^{716-719}$ Nevertheless, a common approach to describe the effect of electron correlations in the opening of such a band gap in systems with half-filled bands has been to use the Hubbard model Hamiltonian, where two competing terms, one favoring electron hopping and another favoring localization, induce a gap opening in the density of states with increasing strength of the intra-site energy $U$. In this picture, two limit cases are distinguished for metal oxides: ${ }^{590,720}$ in one case, typically for light $3 d$ transition metals, the oxygen derived $p$ band lies well below the $3 d$ band and the effect of electron correlations is that of splitting the original $3 \mathrm{~d}$ band into a fully occupied lower Hubbard band and an empty upper Hubbard band, separated by an 
Epitaxial ferroelectric interfacial devices

energy gap of the order of the intra-site energy $U$ (Mott-Hubbard insulators). A second case occurs in oxides with strong $p-d$ hybridization, such as in the late transition metal oxides, where the wavefunction overlap between cations and the oxygen $p$-orbitals is strong and $U$ is larger than the energy separation between the $d$ and $p$ bands; the effect of electron correlations in such case is to split the $d$-band into a filled lower Hubbard band below the (filled) $p$ band and an empty upper Hubbard band separated from the $p$-derived band by an energy gap $\Delta$ (charge transfer insulators). This division underlines the fact that in Mott-Hubbard insulators charge excitation occurs through direct hopping between cation sites while in the charge transfer insulators, charge excitation occurs primarily through the oxygen anions. ${ }^{590}$ In Table VI we list the electronic band parameters of several oxide materials following this picture, mostly derived from x-ray spectroscopy measurements, running from the early to the later transition $3 \mathrm{~d}$ metals collected from the literature, showing a gradual decrease of $\Delta$ and an increase in $U$ as one goes down the table (increasing 3 d atomic number).

At constant temperature, two approaches can be envisaged for inducing an insulator to metal transition in the Mott insulators, namely, through bandwidth modulation or through band filling. ${ }^{590}$ Bandwidth modulation can be achieved by changing the atomic spacing to control the orbital overlap through mechanical or chemical pressure (by substituting isovalent cations within the compound) and thereby affect the strength of electron correlations; in the doped manganites, it can be controlled by applying a magnetic field, where electron hoping is then modified through the double exchange mechanism, ${ }^{741,742}$ a process underlying the so-called "colossal" magnetoresistance (CMR) effect. ${ }^{743-747}$ Band filling consists in directly modulating the charge carrier density, for example, through chemical, electrostatic, or ionic doping. In the case of chemical doping, it is found that the insulating state is stable against relatively large doping contents, an effect that is ascribed to carrier localization induced by static random potential and/or electron-lattice interaction; ${ }^{590}$ doping levels of the order of 0.1-10\% are typically required for a transition into a metallic state to occur. This implies that large charge carrier modulations are required to bring a Mott insulator to the metallic state. Doping by chemical substitution has the disadvantage of being irreversible and of introducing local distortions in the atomic lattice that can further perturb the electronic state. An alternative approach is to use field effects to modulate the carrier density, which is simultaneously reversible and does not introduce local distortions to the lattice. In this approach to controlling the Mott metal insulator transition, the channel layer consists of a 
Epitaxial ferroelectric interfacial devices

TABLE VI. Properties of selected perovskite strongly-correlated oxide materials. BI: band insulator; CT: charge transfer insulator; MH: Mott-Hubbard insulator; NM: non-magnetic; AFM: antiferromagnetic order; NO: not ordered. $T_{\mathrm{c}}$ is the critical temperature for the onset of the magnetic order, $\Delta$ is the $p$ - $d$ charge transfer energy, $U$ is the $d$ - $d$ Coulomb repulsion energy, and $E_{\mathrm{g}}$ is the direct (optical) band gap. Example of typical A-site dopants are given in the last column.

\begin{tabular}{llllllll}
\hline \hline System & Type & Spin order & $T_{\mathrm{c}}(\mathrm{K})$ & $\Delta(\mathrm{eV})$ & $U(\mathrm{eV})$ & $E_{\mathrm{g}}(\mathrm{eV})$ & dopants \\
\hline $\mathrm{SrTiO}_{3}$ & BI & NM & - & $4.0^{\mathrm{l}}$ & $4.5^{\mathrm{l}}$ & $3.75^{\mathrm{a}}$ & $\mathrm{La}, \mathrm{Nb}$ \\
$\mathrm{LaTiO}_{3}$ & $\mathrm{MH}$ & AFM & $140^{\mathrm{b}}$ & $6.0^{\mathrm{m}}$ & $4^{\mathrm{b}}$ & $0.2^{\mathrm{b}}$ & $\mathrm{Sr}, \mathrm{Y}$ \\
$\mathrm{LaVO}_{3}$ & $\mathrm{MH}$ & AFM & $143^{\mathrm{d}}$ & $6^{\mathrm{c}}$ & $4.5^{\mathrm{c}}$ & $1.1^{\mathrm{e}}$ & $\mathrm{Ca}, \mathrm{Sr}$ \\
$\mathrm{LaCrO}_{3}$ & $\mathrm{CT}$ & AFM & $286^{\mathrm{g}}$ & $5.2^{\mathrm{m}}$ & $5.2^{\mathrm{m}}$ & $3.4^{\mathrm{e}}$ & $\mathrm{Ca}, \mathrm{Sr}$ \\
$\mathrm{LaMnO}_{3}$ & $\mathrm{CT}$ & AFM & 140 & $4^{\mathrm{c}}$ & $5.5^{\mathrm{c}}$ & $1.1^{\mathrm{e}}$ & $\mathrm{Ca}, \mathrm{Sr}, \mathrm{Ba}, \mathrm{Pb}$ \\
$\mathrm{LaFeO}_{3}$ & $\mathrm{CT}$ & AFM & $740^{\mathrm{h}}$ & $2.5^{\mathrm{n}}$ & $7.5^{\mathrm{n}}$ & $2.1^{\mathrm{e}}$ & $\mathrm{Ca}, \mathrm{Sr}, \mathrm{Ba}, \mathrm{Bi}$ \\
$\mathrm{LaCoO}_{3}$ & CT & NO & - & $2.0^{\mathrm{p}}$ & $5.5^{\mathrm{p}}$ & $0.3^{\mathrm{e}}$ & $\mathrm{Sr}$ \\
$\mathrm{LaNiO}_{3}$ & M & NO & - & $1.0^{\mathrm{q}}$ & $7.0^{\mathrm{q}}$ & $0^{\mathrm{e}}$ & $\mathrm{O}$ \\
$\mathrm{SrCuO}_{2} \mathrm{j}$ & $\mathrm{CT}$ & AFM & $442^{\mathrm{i}}$ & & & $3.3^{\mathrm{f}}$ & $\mathrm{Ca}, \mathrm{La}, \mathrm{Nd}$ \\
$\mathrm{La}_{2} \mathrm{CuO}_{4}$ & CT & AFM & $325^{\mathrm{s}}$ & $0.3-0.4^{\mathrm{t}}$ & $5-6.5^{\mathrm{t}}$ & $2.0^{\mathrm{k}}$ & $\mathrm{Sr}, \mathrm{Ba}$ \\
$\mathrm{YBa}_{2} \mathrm{Cu}_{3} \mathrm{O}_{7-\delta}$ & CT & AFM & 400 & $0.5^{\mathrm{u}}$ & $5-6.5^{\mathrm{u}}$ & $1.5^{\mathrm{v}}$ & $\mathrm{O}$ \\
\hline \hline
\end{tabular}

${ }^{a}$ Ref. 721

b Ref. 722

${ }^{\mathrm{c}}$ Ref. 723

${ }^{d}$ Ref. 724

${ }^{\text {e }}$ Ref. 725

${ }^{\mathrm{f}}$ Ref. 726

g Ref. 727

${ }^{\mathrm{h}}$ Ref. 728

${ }^{i}$ Ref. 729

${ }^{\mathrm{j}}$ Infinite-layer, high pressure, tetragonal phase of $\mathrm{SrCuO}_{2}$.

${ }^{\mathrm{k}}$ Ref. 730

${ }^{1}$ Ref. 731

${ }^{\mathrm{m}}$ Ref. 732

${ }^{\mathrm{n}}$ Ref. 733

p Ref. 734

${ }^{\text {q }}$ Ref. 735

s Ref. 736

${ }^{\mathrm{t}}$ Ref. 737 and 738

uef. 737 and 738

${ }^{\mathrm{v}}$ For semiconducting $\delta=1$, Ref. 739 and 740 
Epitaxial ferroelectric interfacial devices

Mott insulator chosen with a composition on the phase diagram close to the insulator to metal transition. For maximum effect, the channel layer is made as thin as possible, ideally of the order of the Thomas-Fermi screening length of the metallic phase. One important consideration here is that, due to the epitaxial strain or other interface effects, the phase diagram of the Mott insulator may deviate strongly from that of the bulk. One example is $\mathrm{La}_{1-x} \mathrm{Sr}_{x} \mathrm{MnO}_{3}$, where epitaxial strain strongly affects the transport and magnetic properties in ultrathin films. ${ }^{748,749}$

One motivation for the electrostatic control of the Mott transition has been as an alternative to CMOS field effect transistors, where the main advantages include high on/off resistance ratios (expected at the metal-insulator transition), good scaling characteristics, and the inherent fast dynamics (since the process is purely electronically driven) ${ }^{750-753}$ Prototype Mott transition field effect transistors based on cuprates and using $\mathrm{SrTiO}_{3}$ as the gate dielectric were reported by Newns et al., ${ }^{750,754}$ where control of the conductivity of the channel layer with gate voltage and a quasi-linear variation of the drain current with the drain-source voltage were demonstrated. Unlike in superconducting FETs, the aim here is to control the metal-insulator transition and the measurements were carried out at room temperature. In another example, electrostatic doping of $\mathrm{Sm}_{2} \mathrm{CuO}_{4}$ was achieved by using the band bending characteristics when in contact with a Nb-doped $\mathrm{SrTiO}_{3}$ interface, ${ }^{755}$ an approach that has been extended to other materials systems. ${ }^{756}$ In addition, control of the resistive state of Mott insulators using ionic liquids as the gate dielectric has been reported in the manganites, including control of the electronic state ${ }^{742}$ and of the conduction percolation path. $^{757}$

A large number of studies has been carried out on ferroelectric Mott devices based on several channel systems, including cuprates, ${ }^{758,759}$ manganites, ${ }^{603,760-766}$ nickelates, ${ }^{710,767,768}$ and cobaltates. ${ }^{764}$ Several aspects need consideration. A first aspect is that the resistivity of the doped Mott insulator near the insulator to metallic transition is usually far lower than that of the undoped system. For example, for $\mathrm{La}_{1-x} \mathrm{Sr}_{x} \mathrm{MnO}_{3}$, the $0 \mathrm{~K}$ resistivity for $x=0.175$, in the metallic regime, is $1.0 \times 10^{-3} \Omega \mathrm{cm}$, while for $x=0.15$, in the insulating state, it is $2.0 \times 10^{3} \Omega \mathrm{cm}$, which in turn is orders of magnitude lower than that for $x=0 .{ }^{769}$ As a consequence, screening lengths will be small, requiring small channel layer thicknesses for effective field effect modulation. However, control of the electronic properties of strongly correlated oxides at small thicknesses is challenging, since their properties 
Epitaxial ferroelectric interfacial devices

tend to deviate strongly from the bulk for ultrathin films due to their sensitivity to strain and defects. ${ }^{749,767}$ Another aspect relates to changes in other properties accompanying the metal-insulator transition, such as changes in the magnetic and orbital state and phase segregation as a result of competing ground states. ${ }^{593,770-772}$ For example, the early ferroelectric field effect transistors employed thick manganite layers, $30-50 \mathrm{~nm}$ thick, ${ }^{603,773}$ and the large changes in resistivity found, up to $300 \%$, were interpreted within a percolative phase separation picture. ${ }^{773}$ In another study, on $8.2 \mathrm{~nm} \mathrm{La}_{1-x} \mathrm{Ca}_{x} \mathrm{MnO}_{3}$ films using $\mathrm{SrTiO}_{3}$ as a gate dielectric, an ambipolar effect with the applied electric field was observed, ${ }^{774}$ an effect that was attributed to the development of a pseudogap in the density of states, providing another illustration of the complex behavior of this class of complex oxide materials.

Since large changes in the charge carrier density are required to control Mott insulators, it is advantageous to employ ferroelectric gates with large polarizations. Promising in this regard is $\mathrm{BiFeO}_{3}$ in its supertetragonal phase, characterized by very large spontaneous ferroelectric polarizations in excess of $100 \mu \mathrm{C} / \mathrm{cm}^{2}$ at room temperature, ${ }^{239,372,439}$ and which has been employed for the control of the electric conductivity of $\mathrm{CaMnO}_{3}$ and Ce-doped $\mathrm{CaMnO}_{3} \cdot{ }^{765,766}$ For $\mathrm{CaMnO}_{3}$, large changes in conductivity are obtained, of $400 \%$ at room temperature and a ten-fold change at $200 \mathrm{~K}$, although the system remains in the insulating state for the film thicknesses investigated, in the range from 6-40 unit cells. ${ }^{765}$ With Ce-doping of 0.04 , a metal to insulator transition is observed at around $100 \mathrm{~K} .{ }^{765}$ However, ferroelectric field modulation of the resistivity was found to be much less efficient in this system, which was attributed to incomplete ferroelectric polarization reversal at the interface, as determined from local measurements of the ferroelectric polarization with HR-STEM. ${ }^{766}$

Control of the conductivity of the rare-earth perovskite nickelates, a metallic oxide conductor, has also generated interest. These materials have electrical properties that depend sensitively on the lattice structure and which are strongly affected by the overlap of $\mathrm{Ni} 3 \mathrm{~d}$ and $\mathrm{O} 2 \mathrm{p}$ orbitals, as determined by the Ni-O bond length and $\mathrm{Ni}-\mathrm{O}-\mathrm{Ni}$ bond angle. 775,776 Due to the strong electron-lattice coupling, one route to controlling the electrical properties of the nickelates is by modulating the Ni-O-Ni bond angle using strain ${ }^{777}$ and the surface termination. ${ }^{778,779}$ However, such approaches are limited to static control of the Ni-O-Ni bond angle. In this context, a recent report on the ferroelectric $\mathrm{PbTiO}_{3} / \mathrm{LaNiO}_{3}$ interface suggested a novel way to achieve active control of the electrical properties of the nickelates via interface structure modulation. ${ }^{780}$ In this case, switching the ferroelectric polarization of 
Epitaxial ferroelectric interfacial devices

the $\mathrm{PbTiO}_{3}$ layer modifies the Ni-O bond length and Ni-O-Ni bond angles at the interfacial $\mathrm{LaNiO}_{3}$ layer through ferroelectrically induced structural distortions; the latter contribute to the conductivity change of the $\mathrm{LaNiO}_{3}$ channel by a modification of the mobility, unlike in the canonical field effect. ${ }^{710}$ The result shows that the ferroelectric interface structure modulation can provide a novel way to controlling functionality in oxide electronics.

Strategies for optimizing the changes in the conduction state of Mott insulators have been proposed, such as by combining different materials in bilayer stacks in order to better engineer the interface charge modulation. For example, it is observed that changes in the conductivity of $\mathrm{Sm}_{0.5} \mathrm{Nd}_{0.5} \mathrm{NiO}_{3}$, which exhibits a metal to insulator transition as a thin film grown on $\mathrm{LaAlO}_{3},{ }^{767,768}$ can be enhanced from a $10 \%$ to a $300 \%$ effect when a thin LSMO layer is added at the substrate interface. ${ }^{768}$ In that study, x-ray spectroscopy measurements supported by ab initio calculations showed that between the interfacial Mn and Ni layers a charge transfer from $\mathrm{Mn}$ to $\mathrm{Ni}$ of about 0.1 electron per 2D unit cell takes place, which has the effect of making the system more susceptible to the ferroelectric charge modulation.

In the examples discussed above, large changes in the resistivity of the doped Mott insulators are observed, much higher than expected based on the simple modulation of the charge carrier density, a signature of the role of electron correlations; however, the state of the system tends to remain either insulating or conducting, an indication of the difficulty in controlling the Mott insulator to metallic transition. However, for optimized device structures, a genuine control of the conduction state can be achieved. An example is shown in Fig. 15 for PZT/La $/ \mathrm{La}_{0 .} \mathrm{Sr}_{0.2} \mathrm{MnO}_{3}$, where the $\mathrm{La}_{0.8} \mathrm{Sr}_{0.2} \mathrm{MnO}_{3}$ film thickness is set at the onset of conductivity (3.8 nm or 10 u.c.) and a change between insulating and conducting states is observed as the system is switched between depletion and accumulation states, respectively. ${ }^{761}$

\section{Electric field control of orbital order}

Orbital order refers to the cooperative onset of a preferential occupation of different orbital states which otherwise are degenerate in energy ${ }^{683,781-786}$ When those states carry no orbital moment, such as the $e_{g}$ states, the degeneracy can be lifted by the Jahn-Teller effect (which posits that orbital degeneracy makes the structure of the molecular complex unstable against distortions), ${ }^{787}$ while in the case of states carrying orbital moment, such as the $t_{2 g}$ 
Epitaxial ferroelectric interfacial devices

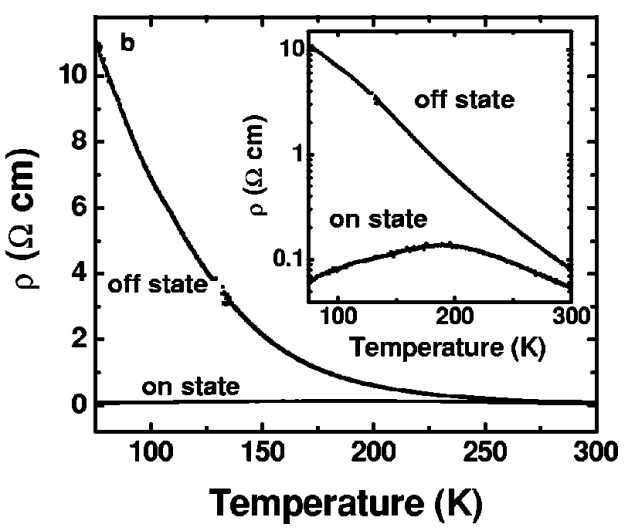

FIG. 15. Resistivity vs. temperature curves for PZT/3.8 nm La ${ }_{0.8} \mathrm{Sr}_{0.2} \mathrm{MnO}_{3}$ heterostructure for the two polarization states of PZT ("off" / "on" is the depletion/accumulation state). The inset shows a lin-log plot of the data. Reproduced from Appl. Phys. Lett. 86, 142501 (2005), with the permission of AIP Publishing.

states, the degeneracy can alternatively be lifted by the spin-orbit coupling. ${ }^{781}$ Long-range orbital order arises from a preferential spatial overlap of neighboring orbitals and is dictated by the sign of the exchange integrals, similar to the situation with the spin Hamiltonian, such that an orbital Hamiltonian can be written in terms of orbital pseudo-spins. ${ }^{781-783}$ Hence, orbital order corresponds to an additional fundamental degree of freedom in the solid state, with its fundamental collective excitation modes, ${ }^{788}$ and whose control constitutes another knob to controlling the electronic state of the system, including the magnetic state to which it is often intimately linked. One example is the orbital arrangement in $\mathrm{LaMnO}_{3}$, where the single electron at the $e_{g}$ level occupies alternately $d_{3 z^{2}-r^{2}}$ or $d_{x^{2}-y^{2}}$ orbitals to form a complex ordered orbital structure that is also linked to the antiferromagnetic spin structure. ${ }^{788}$ Also important in the context of the orbital polarization is the role of strain, and of epitaxial strain in particular, since distortions in the crystal structure can profoundly modify the local crystal field and the position of the atomic energy levels, and therefore, the orbital arrangement.

Although orbital order has been identified in many systems, its control remains challenging. A first hurdle relates to probing orbital order, which typically requires techniques that are sensitive to local orbital arrangement, such as resonant $\mathrm{x}$-ray scattering ${ }^{788,789}$ or $\mathrm{x}$-ray 
Epitaxial ferroelectric interfacial devices

absorption spectroscopy using the x-ray linear dichroic effect, ${ }^{790}$ although it can also be probed indirectly, such as with polarized visible light. ${ }^{791}$ For example, control of the orbital domains in $\mathrm{La}_{1 / 2} \mathrm{Sr}_{3 / 2} \mathrm{MnO}_{4}$ using electric current has been reported, where Joule heating results in melting of charge-orbital ordered domains and the subsequent reduction of the current favouring one particular domain over the other. ${ }^{791}$ Electric-field control of the orbital order has been investigated theoretically using first principles calculations in perovskite vanadate ${ }^{792}$ and $\mathrm{PbTiO}_{3} / \mathrm{LaTiO}_{3}$ superlattices. ${ }^{793}$ In these instances, the polar distortion (induced in the case of the vanadates by a trilinear coupling with antiferrodistortive motions and/or Jahn-Teller distortions) couples with the non-polar ordered Jahn-Teller distortion modes (leading to orbital order), which in turn couple to the spin configuration via the superexchange interaction. ${ }^{792,793}$ In principle, such coupling makes electric field control of orbital and spin ordering in those systems possible.

A related concept, particularly relevant for thin films and interfaces, is that of orbital polarization, which refers to a preferential orbital occupation in the system. ${ }^{27}$ For example, epitaxial strain in thin films can induce a modification in the energy position of the $e_{g}$ orbital states in the manganites and a change in the relative orbital occupancy, i.e., in orbital polarization. ${ }^{749,794-796}$ Control of orbital polarization at the interface between ferroelectrics and complex oxides has been reported for LSMO/BiFeO $3,{ }^{797,798} \mathrm{LSMO}_{\mathrm{BaTiO}},{ }^{160,799}$ and LSMO/PZT ${ }^{800}$ For the case of $\mathrm{LSMO} / \mathrm{BiFeO}_{3},{ }^{797,798}$ a modification of the orbital occupancy of the $3 \mathrm{~d}$ states at the interface was inferred from x-ray linear dichroic effect measurements due to hybridization between the Mn and Fe orbitals and leading to the presence of a large interfacial magnetic moment in $\mathrm{BiFeO}_{3}$, exchange-coupled to the $\mathrm{La}_{0.8} \mathrm{Sr}_{0.2} \mathrm{MnO}_{3}$ magnetization; however, in these studies, the effect of switching the ferroelectric polarization of $\mathrm{BiFeO}_{3}$ was not addressed, nor the extent of the modified orbital polarization. These latter aspects were studied by Chen et al. ${ }^{799}$ and Preziosi et al. ${ }^{800}$ for $\mathrm{La}_{0.8} \mathrm{Sr}_{0.2} \mathrm{MnO}_{3} / \mathrm{BaTiO}_{3}$ and $\mathrm{La}_{0.825} \mathrm{Sr}_{0.175} \mathrm{MnO}_{3} / \mathrm{PZT}$, respectively. In the first study, the change in orbital polarization was investigated by first principles calculations, predicting the occurrence of a large change in orbital polarization when going from the accumulation state (which favors occupation of the $d_{x^{2}-y^{2}}$ states) to the depletion state (which favors $d_{3 z^{2}-r^{2}}$ states) over a region extending to 3 unit cells from the ferroelectric interface (Fig. 16A). The calculations also predict that the orbital polarization depends only modestly on the ferroelectric polarization amplitude and that the main effect results from a displacement in the atomic position of the $\mathrm{Mn}$ cations 
Epitaxial ferroelectric interfacial devices

induced by the ferroelectric polar displacements (Fig. 16B,C), a result that was confirmed experimentally by TEM measurements of the same system (Fig. 16D). ${ }^{799}$ Hence, the change in orbital occupation is determined by distortions in the Mn octahedron that, in turn, modulate the energy position of the two $e_{g}$ bonding states. These results were corroborated by x-ray linear dichroism measurements reported for $\mathrm{La}_{0.825} \mathrm{Sr}_{0.175} \mathrm{MnO}_{3} / \mathrm{PZT}$, where a change in orbital polarization of $1.5 \%$ averaged over the whole LSMO film is observed (but expected to be significantly higher in the interface region). ${ }^{800}$ Multiplet calculations suggest as well an inversion in the order of the $d_{x^{2}-y^{2}}$ and $d_{3 z^{2}-r^{2}}$ states when switching the ferroelectric polarization (where the latter are favoured for the depletion state), pointing also here to a modulation in the Mn octahedron structure (see Fig. 17). Changes in the orbital moment of $\mathrm{Mn}$ in $\mathrm{La}_{0.67} \mathrm{Sr}_{0.33} \mathrm{MnO}_{3} / \mathrm{BaTiO}_{3}$ structures where the direction of the ferroelectric polarization was pre-set by controlling the substrate termination, were also observed and quantified by using XMCD sum rules ${ }^{801-805}$ at the $\mathrm{Mn} \mathrm{L}_{2,3}$-edge, providing another demonstration of ferroelectric control of the orbital population. ${ }^{160}$

\section{Electric field control of charge order}

In systems with mixed cation valency, a reduction in Coulombic energy can be achieved at half or $1 / 8$ doping through ordered charge localization (charge-ordered state), manifested by in an electrically insulating state below a critical temperature. The paradigm system where charge ordering was first described is magnetite (Verwey transition), ${ }^{806-810}$ while more recently, charge ordering has been reported in another ferrite system, $\mathrm{LuFe}_{2} \mathrm{O}_{4},{ }^{811}$ in the doped manganites such as $\mathrm{La}_{1-x} \mathrm{Ca}_{x} \mathrm{MnO}_{3},{ }^{812-815} \mathrm{PrCaMnO}_{3},{ }^{816-818}$ and $\mathrm{BiSrCaMnO}_{3},{ }^{819-821}$ and in the cuprates, where charge order is thought to be a ubiquitous phenomenon (here most often seen as ordered charge segregation) ${ }^{615,822}$ Probing and establishing charge order in a system is in general challenging; the early work on the manganites cited above relied on neutron scattering measurements, which are not directly sensitive to charge, but rather to lattice modulations induced by doping, ${ }^{823}$ while interpretation of resonant x-ray scattering measurements, which are in principle highly sensitive to charge order, have not been without controversy ${ }^{824,825}$ and still largely rely on models to interpret the experimental results. ${ }^{826}$ For example, a pure ionic picture for charge ordering in the manganites would imply that the x-ray absorption spectrum should consist of a superposition of $\mathrm{Mn}^{3+}$ and $\mathrm{Mn}^{4+}$, while 
Epitaxial ferroelectric interfacial devices
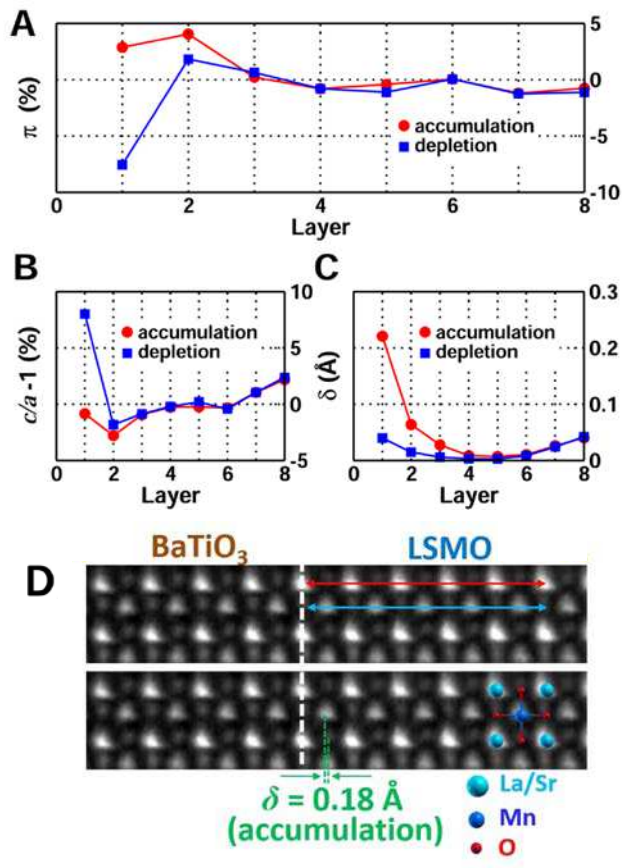

FIG. 16. A-C: $\mathrm{Ab}$ initio results showing, respectively, the variation in orbital polarization, $c / a$ ratio and $\mathrm{Mn}$ cation displacement $\delta$ as a function of atomic position in an $\mathrm{La}_{0.8} \mathrm{Sr}_{0.2} \mathrm{MnO}_{3}$ (LSMO) film from the $\mathrm{BaTiO}_{3}$ interface for the two directions of the $\mathrm{BaTiO}_{3}$ ferroelectric polarization. D: Scanning transmission electron microscopy results for the $\mathrm{La}_{0.8} \mathrm{Sr}_{0.2} \mathrm{MnO}_{3} / \mathrm{BaTiO}_{3}$ interface (accumulation state), demonstrating the presence of a cation displacement at the interface region. Adapted with permission from Nano Letters 14, 4965 (2014). Copyright 2014 American Chemical Society.

the observed spectrum is found to correspond to that of a mixed valency state ${ }^{827-832}$ instead, x-ray spectroscopy and neutron scattering measurements suggest the presence of two Mn sites with different magnetic moments and a difference in valency (charge disproportionation) of $\sim 0.2$ electrons, ${ }^{825,826,828,830,833,834}$ much less than the expected difference of 1 electron between $\mathrm{Mn}^{3+}$ and $\mathrm{Mn}^{4+}$. A similarly complex picture has emerged for magnetite, where the initial ionic model for charge ordering proposed by Verwey ${ }^{806-809}$ was found not to be consistent with the results of detailed x-ray diffraction and x-ray resonant scattering 
Epitaxial ferroelectric interfacial devices

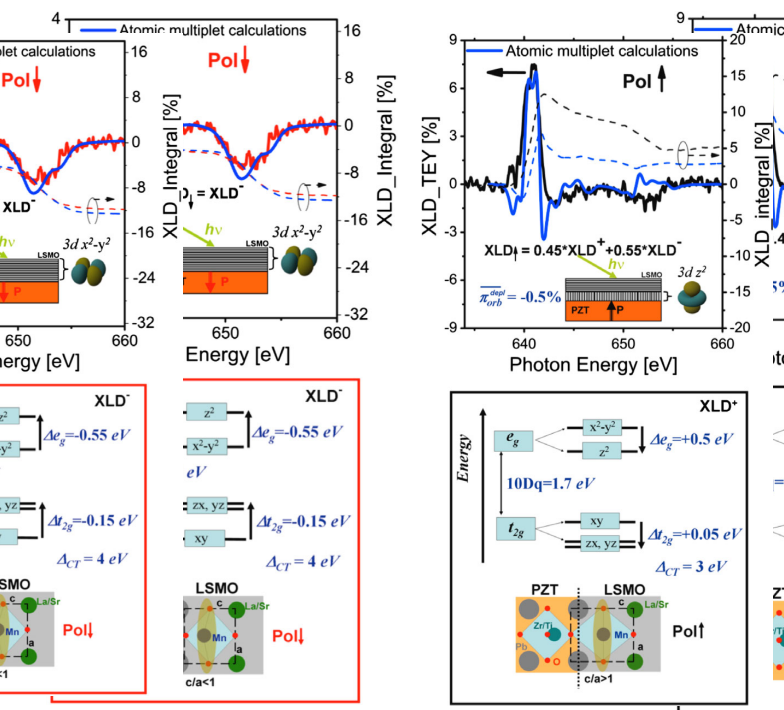

FIG. 17. Experimental and multiplet calculations of the x-ray linear dichroism measured in $\mathrm{La}_{0.825} \mathrm{Sr}_{0.175} \mathrm{MnO}_{3} / \mathrm{PZT}$ for the two direction of the PZT ferroelectric polarization. The bottom panels show the schematic ionic model with the changes in the relative energy position of the 3d $e_{g}$ states in the two cases. Reproduced with permission from Phys. Rev. Lett. 115, 157401 (2015). Copyright 2015 by the American Physical Society.

measurements. ${ }^{835-837}$ In fact, our understanding of magnetite and of the Verwey transition has significantly improved over the last decade. ${ }^{835,836,838-841}$ The current understanding is that, at the Verwey temperature, a structural transition from cubic to monoclinic occurs, characterized by a complex lattice distortion that leads to an increase by $\sqrt{2} \times \sqrt{2} \times 2$ of the high temperature cubic unit cell together with a charge localization process whereby the double-exchange active octahedral Fe cations cluster in a superstructure of trimeron complexes with charge and orbital order. ${ }^{835,836,841}$ The iron valence state varies by 0.6 electrons from +2.35 to +2.95 in contrast to a mixed $3+$ and $2+$ valence state. This variation is for octahedral sites belonging to the trimeron complex. ${ }^{835}$

Given the challenge of probing charge order directly, it is perhaps not surprising that only a few studies have attempted to control charge order using external excitations. Examples include the ultrafast excitation of $\mathrm{La}_{1-x} \mathrm{Ca}_{x} \mathrm{MnO}_{3}$ thin films using high intensity $100 \mathrm{fs}$ 
Epitaxial ferroelectric interfacial devices

laser pulses, where the superlattice reflection associated with orbital/charge order was used to follow the temporal evolution of the system after the excitation; ${ }^{842}$ under such strong excitation conditions, melting of the orbital/charge order is found to occur on a time scale faster than $200 \mathrm{fs}$. Another example is the report of a large birefringence effect that is ascribed to charge order in magnetite, controlled by cooling the system under an applied magnetic field. ${ }^{843}$ In this study, an intense ultra-fast laser pulse was used to achieve a rapid heating of the system to above the Verwey temperature, with the magnetic field setting a preferential orientation for the monoclinic $c$ axis, ${ }^{844}$ and consequently, of charge order; the fast time response of polarization rotation angle was also used to study the dynamics of charge-order melting, found to be faster than $0.8 \mathrm{ps}^{843}$

\section{E. Electric field control of magnetism}

Electric field control of magnetism in the solid state has generated much attention recently, ${ }^{37,845-855}$ motivated by the interest in understanding better the physical processes that lead to a coupling between spin and charge degrees of freedom and also by its potential for applications in next generation electronic devices. ${ }^{344,856-858}$ In magnetoelectric and in multiferroic materials with magnetic and ferroelectric order, such coupling is intrinsic to the system and intimately linked to the specific origin of ferroelectric polarization. ${ }^{33,36,37,40,845,847,852,859-861}$ Although exciting materials to study from a basic science level, the potential for applications is severely limited by their scarcity in nature (a consequence of the required time reversal and inversion broken symmetries together with dielectric behavior for ferroelectricity) ${ }^{32,33,862,863}$ and the generally small value of the effective magnetoelectric coupling or critical temperatures, with $\mathrm{BiFeO}_{3}$ being one outstanding exception. ${ }^{365,369,864-866}$ In order to circumvent such limitations, an alternative route to achieving a strong coupling between magnetic and ferroelectric order parameters consists in designing artificial multiferroic systems obtained by interfacing magnetic with ferroelectric materials. ${ }^{846,848,850,854,867-869}$ To reach large magnetoelectric couplings, both the interface characteristics and the coupling mechanism need to be engineered judiciously. Several interfacial magnetoelectric coupling mechanisms have been devised according to the main interaction process exploited: strain, spin exchange, or charge. ${ }^{848,850}$ Strain-mediated coupling relies on the elastic coupling to modify the magnetic state via magnetoelastic and 
Epitaxial ferroelectric interfacial devices

piezoelectric effects, ${ }^{870-873}$ while spin exchange-mediated magnetoelectric coupling relies on magnetic exchange interactions, for example, between a ferromagnet and an antiferromagnetic multiferroic via the exchange-bias effect. ${ }^{343,345,348,349,848,874-877}$ More directly related to field effects is the charge-mediated magnetoelectric coupling, whereby the magnetic state of the system is modified by changing the charge carrier density by using charge screening at the ferroelectric boundary to accumulate or deplete charge carriers at the metallic interface. We consider next materials whose charge screening length lie within three distinct regimes: (i) metallic ferromagnets, characterized by very short Thomas-Fermi screening lengths due to the very large electron charge density at the Fermi level; (ii) 3d correlated systems, with screening lengths and electron mean free paths of the order of the unit cell, such as mixed valence $3 \mathrm{~d}$ transition metal oxides; and (iii) magnetic semiconductors, where charge screening occurs over the characteristic Debye length $L_{\mathrm{D}}=\sqrt{\epsilon_{\mathrm{s}} \epsilon_{0} k_{\mathrm{B}} T /\left(q^{2} n\right)},{ }^{189}$ characterized by extended charge screening lengths $\left(\epsilon_{\mathrm{s}}\right.$ is the static dielectric constant, $q$ the carrier charge, and $n$ the charge carrier density), typically in the nanometer range (ca. 2-20 nm for carrier densities in the range from $10^{18}$ to $10^{16} \mathrm{~cm}^{-3}$ at room temperature).

\section{Metallic ferromagnets}

In metallic ferromagnets, such as the $3 \mathrm{~d}$ transition metals, the exchange interaction responsible for magnetic order originates from the Pauli exclusion principle and the condition for antisymmetry of the electronic wave function, which directly links the exchange integral term (electron-electron Coulomb energy) to the spin state of the system. ${ }^{626,878}$ The Pauli exclusion principle effectively acts to increase electron-electron separation, reducing the Coulomb energy but at a cost of kinetic energy; hence, only in systems where the density of states at the Fermi energy is high it becomes advantageous for a spin-polarized state to emerge, such as in late $3 \mathrm{~d}$ transition metal elements. The high charge carrier density together with quasi-free electron behavior leads to extremely short Thomas-Fermi screening lengths, of below $1 \AA$. As a consequence, electrostatic control of magnetism, such as of the exchange interaction or the magnetic moment, which entails a shift in the relative occupancy of the spin up and down subbands, ${ }^{879}$ may be expected to be modest except for the thinnest films. Such modifications in the interfacial magnetic moment have been estimated for bcc $\mathrm{Fe}(001), \mathrm{Ni}(001)$, and hcp $\mathrm{Co}(0001)$ when subjected to a static electric field 
Epitaxial ferroelectric interfacial devices

applied across a vacuum ${ }^{880}$ or dielectric barrier. ${ }^{881,882}$ However, modulation of the charge carrier density at the interface can modify strongly the surface magnetic anisotropy via spin-orbit coupling, as a result of a change in the relative electron occupancy of the interfacial orbital states. ${ }^{880,882-888}$ The impact of the surface magnetocrystalline anisotropy can be very strong in $3 \mathrm{~d}$ transition metal ferromagnetic films and nanoparticles, often dominating the magnetic energy. ${ }^{889-892}$ Effectively, the control of the surface magnetic anisotropy through charge screening has been demonstrated recently in a number of systems, including $\mathrm{Fe},{ }^{884} \mathrm{FePt},{ }^{893} \mathrm{Co}_{40} \mathrm{Fe}_{40} \mathrm{~B}_{20},{ }^{894-896} \mathrm{Co},{ }^{897,898} \mathrm{Ni}^{899} \mathrm{Fe}_{0.5} \mathrm{Pd}_{0.5},{ }^{900} \mathrm{Fe}_{80} \mathrm{Co}_{20},{ }^{901-903}$ and in

$\mathrm{Fe}_{80} \mathrm{Co}_{20} / \mathrm{MgO} / \mathrm{Fe}^{904,905}$ and $\mathrm{Fe}_{40} \mathrm{Co}_{40} \mathrm{~B}_{20} / \mathrm{MgO} / \mathrm{Fe}_{40} \mathrm{Co}_{40} \mathrm{~B}_{20}$ tunnel junctions. ${ }^{906,907} \mathrm{~A}$ motivation in this context is the electric field control of the relative magnetization alignment of ferromagnetic layers in spin-valve device structures and of the electrical resistivity through the giant (GMR) or tunneling magnetoresistance (TMR) effects for applications in nonvolatile random access memories (MRAM) and logic switches. ${ }^{344,858,908}$

Additionally, chemical bonding (or hybridization) at the interface can also play an important role. For instance, density functional theory calculations for $\mathrm{Fe} / \mathrm{BaTiO}_{3}(001)$ show a large surface magnetoelectric response whose origin is largely attributed to changes in the chemical bonding at the interface, i.e., to modulations in the Ti $3 d$, Fe $3 d$ and $\mathrm{O} 2 p$ orbital overlap upon reversal of the ferroelectric polarization direction due to the displacement of the Ti atoms, which additionally become magnetically polarized. ${ }^{909-914}$ Similar effects have been calculated for $\mathrm{Co} / \mathrm{PZT},{ }^{915}$ and also for oxides such as $\mathrm{LSMO} / \mathrm{BaTiO}_{3},{ }^{160,799}$ suggesting that such a process may be quite general at ferroelectric interfaces. In fact, magnetic polarization of the Ti interface layer in contact with ferromagnetic systems has been observed in several systems, including $\mathrm{Fe} / \mathrm{BaTiO}_{3}(001),{ }^{916} \mathrm{LSMO} \mathrm{BaTiO}_{3},{ }^{160,917}$ and $\mathrm{Co} / \mathrm{PZT},{ }^{918}$ turning the interface region of the ferroelectric also ferromagnetic, i.e., multiferroic.

\section{Mixed valence transition metal oxides}

In the $3 \mathrm{~d}$ transition metal oxides, the magnetic exchange interaction between cations is indirect, mediated by the oxygen anions, either through virtual excitations of spin up-down pairs to neighboring cations in the case of superexchange interaction (which tends to favour antiferromagnetic coupling) $)^{919}$ or through electron hopping in the case of double exchange interaction, which favors ferromagnetic coupling. ${ }^{741,920-922}$ Although indirect, magnetic in- 
Epitaxial ferroelectric interfacial devices

teractions in many oxides can be strong and result in high magnetic critical temperatures, for example, up to $858 \mathrm{~K}$ for the ferrimagnetic spinels $\mathrm{NiFe}_{2} \mathrm{O}_{4}$ and $\mathrm{Fe}_{3} \mathrm{O}_{4} \cdot{ }^{840,923,924}$ In comparison to magnetic metals and magnetic semiconductors, complex oxides tend to exhibit stronger electron-electron correlations and a more diverse electronic behavior as a function of charge carrier density, where magnetism competes or co-exists with other types of order, such as orbital order or superconductivity, leading to complex phase diagrams. ${ }^{590,593,784,925-927}$ Typically, the effect of doping in the $3 \mathrm{~d}$ metal oxides, for example via chemical substitution, is that of modifying the average valence state of the $3 \mathrm{~d}$ cations and modifying the charge carrier density. The main approach to electrostatic control of magnetism in such mixedvalence systems is to choose a chemical doping that places the system at a phase boundary between two magnetic ground states of the system and to drive it across the boundary by electrostatic modulation of the charge carrier density. Also here it is important to recall that the phase diagram of thin films can deviate significantly from that of the bulk material, for example, due to epitaxial strain, as illustrated by the changes in the magnetic and conducting state of $\mathrm{La}_{1-x} \mathrm{Sr}_{x} \mathrm{MnO}_{3}$ films near 0.5 doping with strain shown in Fig. 18. ${ }^{748,749}$

Among the mixed valence materials, $3 \mathrm{~d}$ metal oxides crystallizing in the perovskite structure have generated notable interest due to their diverse and important chemical and physical properties. ${ }^{928-931}$ Within this class of materials, the manganites have been intensively investigated, ${ }^{932,933}$ particularly since the observation of the CMR effect, ${ }^{743-747}$ effectively, a magnetic field-driven insulator to metal transition. The phase diagrams of the manganites are characterized by several phase transitions as a function of chemical doping, for example, for $\mathrm{La}_{1-x} \mathrm{Sr}_{x} \mathrm{MnO}_{3}$, from an insulating orbital ordered antiferromagnetic $(x=0)$, to insulating ferromagnetic $(0<x<0.2)$, metallic ferromagnetic $(0.2<x<0.5)$, metallic antiferromagnetic $(x=0.5)$ to antiferromagnetic insulating $(x>0.5) .{ }^{769,934,935}$ In many manganite perovskites, the equilibrium phases near the boundaries are quasi-degenerate in energy and coexist, ${ }^{593,770-772}$ suggesting that the equilibrium state of the system can be tilted towards one or the other state with only modest perturbations. The charge carrier density at the boundaries separating such phase transitions is high, typically in the range of $10^{19}-10^{22} \mathrm{~cm}^{-3}$, requiring a correspondingly large charge carrier modulation for controlling the equilibrium state. ${ }^{3}$ In addition, the system should be as thin as possible for maximal field effect, ideally, of the order of the charge screening length. Experimentally, it is known that the magnetic and electrical properties of thin films of the manganites grown on $\mathrm{SrTiO}_{3}$ 
Epitaxial ferroelectric interfacial devices

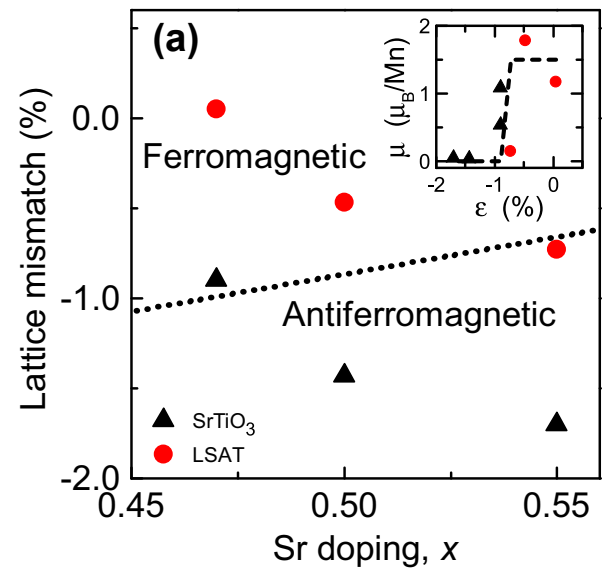

FIG. 18. Diagram of the magnetic state of the LSMO films grown on LSAT (circles) and $\mathrm{SrTiO}_{3}$ (triangles) as a function of lattice mismatch $(\epsilon)$ and doping. Dotted line separates ferromagnetic from antiferromagnetic ground states. Inset shows the variation of the magnetic moment, $\mu$, vs. $\epsilon$ (dashed line is guide to the eye). Reproduced with permission from Phys. Rev. B 90, 024414 (2014). Copyright 2014 by the American Physical Society.

are strongly depressed below a thickness of 1-3 nm, ${ }^{862,936-944}$ while epitaxial strain is also known to strongly affect the magnetic and electrical properties (Fig. 18). ${ }^{748,749}$ Hence, the strategy for modulating the magnetic properties of the mixed valence manganites using the ferroelectric field effect has been to setting the thickness of the manganite layer at the transition between insulator to metallic state; for example, for $\mathrm{La}_{1-x} \mathrm{Sr}_{x} \mathrm{MnO}_{3} / \mathrm{SrTiO}_{3}(001)$ at $x=0.2$ and $x=0.3$, this occurs at a film thickness of about 10 unit cells. ${ }^{760,940,945}$ Using this approach, demonstration of electric field control of the magnetic properties of the doped manganites in ferroelectric field effect structures could be achieved, including modulations in the critical temperature, ${ }^{760,761}$ anisotropic magnetoresistance ${ }^{596}$ and in the magnetic polarization. ${ }^{946}$

An important breakthrough was the experimental report of a large modulation of the coercivity, magnetic critical temperature, and magnetic moment of a $4 \mathrm{~nm} \mathrm{La} 0.8 \mathrm{Sr}_{0.2} \mathrm{MnO}_{3} / \mathrm{PZT}$ heterostructure as a function of the applied electric field measured by magneto-optic Kerr effect (MOKE) magnetometry, demonstrating the presence of a large magnetoelectric cou- 
Epitaxial ferroelectric interfacial devices

pling in this artificial multiferroic system that was both hysteretic and non-volatile. ${ }^{317}$ The results are in qualitative agreement with what is expected from the bulk phase diagram of $\mathrm{La}_{1-x} \mathrm{Sr}_{x} \mathrm{MnO}_{3},{ }^{769,934,935}$ including a higher critical temperature and lower magnetic moment with increasing doping. Subsequent x-ray absorption near-edge spectroscopy (XANES) measurements showed that switching the ferroelectric polarization resulted in a shift in the absorption edge of the Mn K-edge by about $0.3 \mathrm{eV}$, interpreted as a change in the valence state of the interfacial Mn layer at the PZT interface when switching between the accumulation and depletion states. ${ }^{315,945}$ Such measurements demonstrated the electronic origin of the magnetoelectric effect in PZT/LSMO and allowed a quantitative comparison of the changes in the magnetic moment expected from a simple modulation of the $\mathrm{Mn}$ valence state (of $0.1 \mu_{\mathrm{B}} / \mathrm{Mn}$ ) and that measured experimentally $\left(0.76 \mu_{\mathrm{B}} / \mathrm{Mn}\right)$, showing that an additional change in the spin configuration at the interface takes place, from a ferromagnetic coupling in the depletion state to an antiferromagnetic coupling in the accumulation state, and attributed to a change in the relative weights of the superexchange and double exchange energy terms as a function of the $3 \mathrm{~d} z^{2}$ orbital occupancy induced by screening. The presence of such a magnetic reconstruction at the interface is supported by the results of first principles calculations. ${ }^{947-953}$ Also significant, the hole accumulation state shows a much higher critical temperature than the depletion state (by about $20 \mathrm{~K}$ ), such that over a large temperature window on/off switching of magnetism driven by the orientation of the ferroelectric polarization is realized. ${ }^{954,955}$ Subsequent studies reported similar modulations of the magnetization in $\mathrm{La}_{1-x} \mathrm{Sr}_{x} \mathrm{MnO}_{3}$-ferroelectric heterostructures at various doping levels and employing different ferroelectric systems, examples of which are summarized in Table VII, showing that the magnetoelectric effect is not very sensitive to the exact amplitude of the ferroelectric polarization and that charge modulations in the range between $25-100 \mu \mathrm{C} / \mathrm{cm}^{2}$ at the LSMO interface are sufficient to modify the interfacial magnetic state. However, a number of discrepant results for the magnetic and electronic behavior has been observed, such as the case for $\mathrm{BaTiO}_{3} / \mathrm{LSMO} / \mathrm{SrTiO}_{3}(001)$ at $x=0.3$ doping, where an opposite change in the saturation moment with the direction of the ferroelectric polarization is observed and no change in the critical temperature ${ }^{956}$ an effect attributed to a modulation in the relative population of the ferromagnetic metallic and antiferromagnetic insulating phases coexisting in the system. One advantage of $\mathrm{La}_{1-x} \mathrm{Sr}_{x} \mathrm{MnO}_{3}$ at $x=0.3$ doping resides in its relatively high bulk Curie temperature, of about $370 \mathrm{~K}$, such that room temperature 
Epitaxial ferroelectric interfacial devices

demonstration of a large change in the magnetic moment would be of high practical interest.

TABLE VII. Magnetoelectric coupling in manganite-ferroelectric heterostructures. *Estimated using 1 u.c. $=3.9 \AA .{ }^{* *}$ With a $\mathrm{BiO}-\mathrm{MnO}_{2}$ interface stacking; †with a $\mathrm{FeO}_{2}$ - $(\mathrm{La}, \mathrm{Sr}) \mathrm{O}$ interface stacking. Different ferroelectric polarization states achieved by $\ddagger$ changing substrate termination or §using the flexoelectric effect. IUsing the conversion ratio for the neutron magnetic scattering length of $10^{-6} \mu_{\mathrm{B}} / \AA^{2}$ for LSMO from Ref. 957.

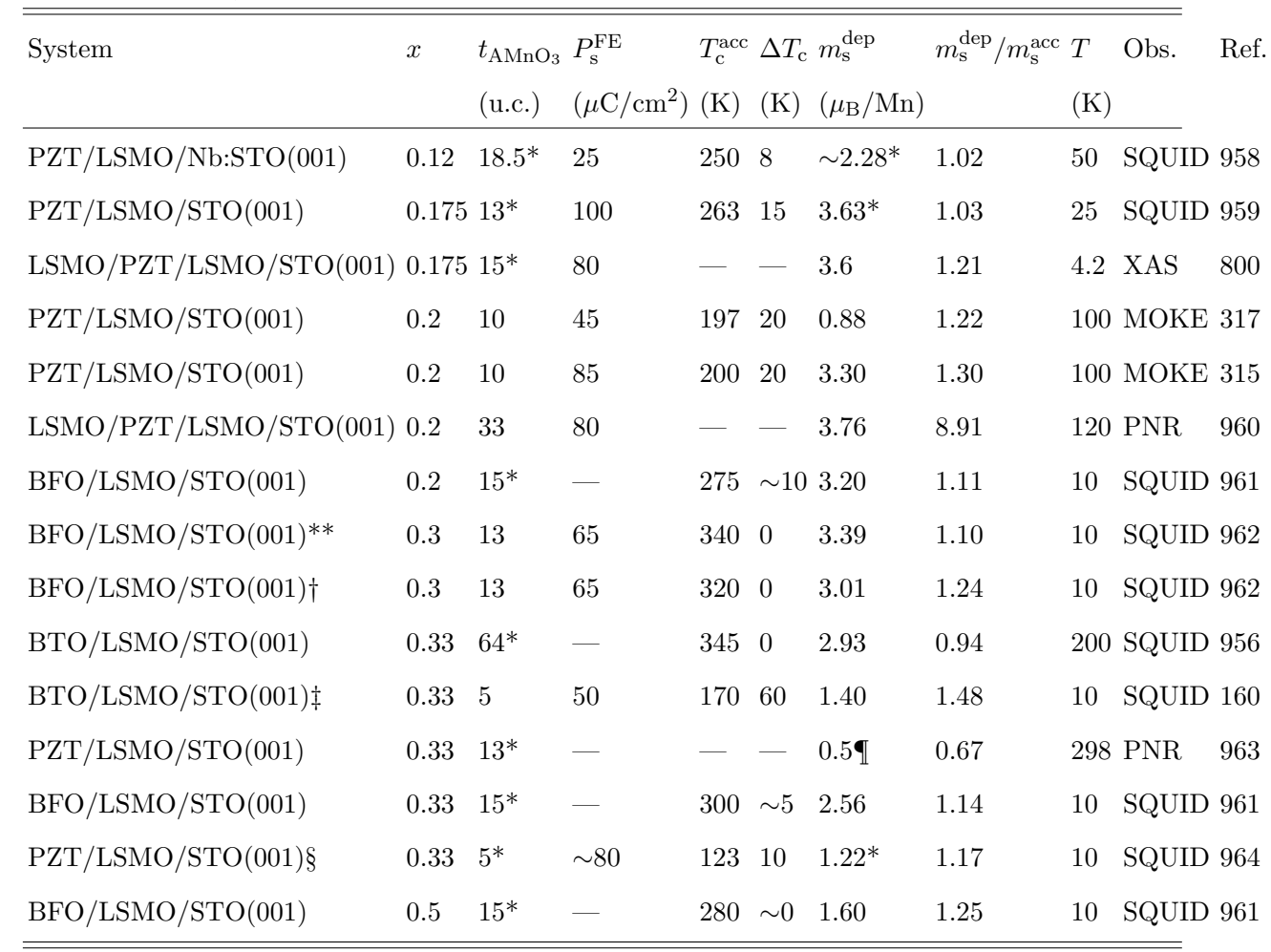

The exact interfacial spin configuration in the different types of heterostructures discussed above still remains to be fully elucidated, in particular, since several possibilities for the ground state of the system in the accumulation state have been predicted theoretically. $2,948,949,951,952$ It is clear that to determine the interfacial spin configuration in such heterostructures requires local probes of the spin configuration. For example, Ma et al. ${ }^{965}$ probed the interfacial magnetic signal of PZT/LSMO/LAO $(001)$ at $x=0.3$ doping 
Epitaxial ferroelectric interfacial devices

with second harmonic signal generation to show that the second harmonic signal is absent in the accumulation state (no net magnetization, suggesting an interfacial antiferromagnetic state) and present in the depletion state (corresponding to a uniform ferromagnetic state across the interface), consistent with the results for PZT/LSMO/STO(001) at $x=0.2$ described above (although the strain state of the LSMO film may differ in the two cases). ${ }^{315,317}$ Another local probe of magnetism is polarized neutron reflectivity (PNR), which enables one to measure the magnetization profile of buried films thanks to the high penetration depth of neutron beams and the intrinsic magnetic moment of the neutron. ${ }^{966-970}$ The study by Meyer et al. ${ }^{960}$ on various $\mathrm{LSMO} / M / \mathrm{LSMO} / \mathrm{SrTiO}_{3}(001)(x=0.2)$ heterostructures, with $M=\mathrm{PZT}$ or LAO, could identify strongly varying magnetic moments in the LSMO films as the layer structure grows in complexity (i.e., from the single $\mathrm{La}_{0.8} \mathrm{Sr}_{0.2} \mathrm{MnO}_{3}$ layer to the full multilayer stack), showing that adding a capping layer to the LSMO enhances the magnetic moment in the bulk of the film and that the presence of a ferroelectric interface enhances the magnetic moment in the charge-depleted interface and reduces it in the charge-accumulated interface (both in absolute terms and also with respect to the bulk film magnetic moment); the control sample where PZT is replaced by a non-ferroelectric $\mathrm{LaAlO}_{3}$ film still shows a reduction of the moment of the top (uncapped) LSMO film but no moment enhancement at the $\mathrm{LaAlO}_{3} / \mathrm{La}_{0.8} \mathrm{Sr}_{0.2} \mathrm{MnO}_{3}$ interface, as shown in Fig. 19. The results of a very recent study by Liu et al. ${ }^{964}$ combining electrical, magnetic, spectroscopic, and neutron transport (PNR) characterisation are also included in Table VII for PZT/LSMO $(x=0.33)$, where the direction of the PZT ferroelectric polarisation was configured via the flexoelectric effect. In this case, different LSMO films were prepared with the PZT polarisation pointing into or away from the LSMO interface; the LSMO films at $2 \mathrm{~nm}$ thickness were found to exhibit the strongest magnetolectric effects and consistent with the results for 0.2 doping; in particular, the films at this thickness are found to exhibit two magnetic transitions, the lower ones associated to the presence of the ferroelectric layer. By using PNR to evaluate the magnetic profile of the two $2 \mathrm{~nm}$ LSMO films with different polarisation orientations, the authors find the presence of an appositely aligned interfacial magnetic layer for the accumulation state and a slightly enhanced magnetic moment for the depletion state, in agreement with the model proposed by Vaz et al. ${ }^{315}$ and in agreement with DFT calculations. ${ }^{948,950-953}$ The XAS results indicate that while the depletion state favours the occupation of $3 z^{2}-r^{2}$ states, the accumulation state favours occupation of $x^{2}-y^{2}$ states and an antiferromagnetic coupling 
Epitaxial ferroelectric interfacial devices

with the dominant $x^{2}-y^{2}$ states in the bulk of the films, favoured by epitaxial strain. ${ }^{964}$ In another PNR study of a PZT/LSMO $(x=0.33)$ bilayer structure, ${ }^{963}$ the PNR spectra were taken for the two polarization states of the PZT, where the ferroelectric polarization was switched by using a Pt electrode deposited on the top surface of the PZT film. The change in the magnetization profile shows a large reduction of the interfacial moment of LSMO when going from the accumulation to the depletion state, i.e., opposite to that found for LSMO at $x=0.2$ doping. The measurements were carried out while the electric field was applied to the PZT layer and although one could expect the strain state of the PZT to be identical for both states of the ferroelectric polarization, x-ray diffraction measurements of the out of plane lattice parameter of the PZT layer indicate an asymmetric behavior for positive and negative voltages, such that the observed magnetic changes are explained as a combination of charge and strain effects; the results of ab initio calculations support the conclusion that strain is the leading factor responsible for the observed experimental behavior. ${ }^{963}$

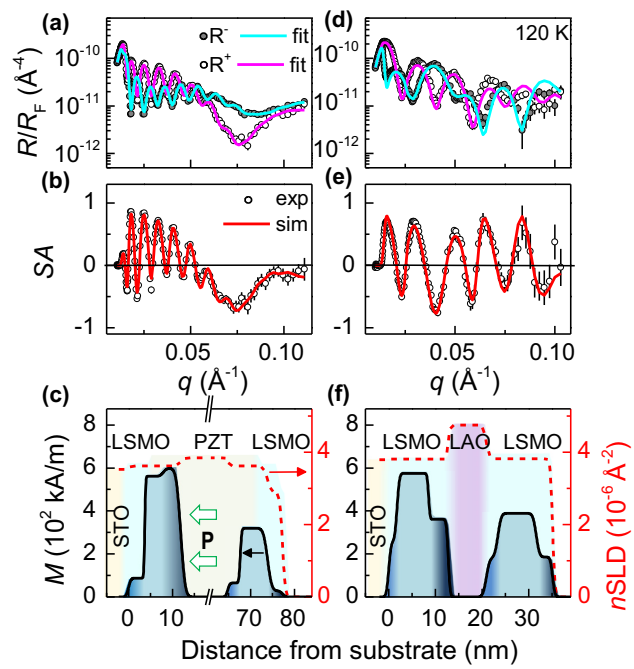

FIG. 19. Polarized neutron reflectivity spectra and magnetic depth profiles of (a-c) LSMO/PZT/LSMO/STO(001) and (d-f) LSMO/LAO/LSMO/STO(001) heterostructures obtained from fits to PNR data. Reproduced with permission from Phys. Rev. B 94, 174432 (2016). Copyright 2016 by the American Physical Society.

In a detailed study of the spin and orbital state of 5 u.c. $\mathrm{La}_{2 / 3} \mathrm{Sr}_{1 / 3} \mathrm{MnO}_{3} / \mathrm{BaTiO}_{3}$ het- 
Epitaxial ferroelectric interfacial devices

erostructures by XAS and HR-STEM, Wang et al. ${ }^{160}$ have observed a dependence of both magnetism and orbital polarization in both $\mathrm{LSMO}$ and $\mathrm{BaTiO}_{3}$ interfacial layers with the direction of the ferroelectric polarization. Samples with different directions of the ferroelectric polarization were prepared by engineering the interface of the $\mathrm{SrTiO}_{3}(001)$ substrate, by either using a $\mathrm{SrO}$ termination, which favors the state with the polarization pointing away from the $\mathrm{SrTiO}_{3}$ interface, or using a $\mathrm{TiO}_{2}$ surface termination, which favors the opposite polarization direction. The results of magnetic x-ray absorption spectroscopy at the Mn Ledge show a stronger magnetic signal for the state pointing away from the $\mathrm{La}_{1-x} \mathrm{Sr}_{x} \mathrm{MnO}_{3}$, corresponding to depletion of hole carriers from the $\mathrm{La}_{1-x} \mathrm{Sr}_{x} \mathrm{MnO}_{3}$ interface, compared to the accumulation state; in addition, a similar increase in the magnetic signal amplitude is observed at the Ti L-edge, albeit with a change in signal for the depletion state, as shown in Fig. 20. Interestingly, using the XMCD sum rules to estimate spin and orbital components of the magnetic moment, they find that the orbital moment is significant for the depletion state, but zero for the accumulation state. These results were explained in terms of a change in the magnetic configuration from ferro- to antiferromagnetic when going from the depletion to accumulation state, in the latter case, supported by the observation of a small exchange bias in the system (of up to 20 Oe at $10 \mathrm{~K}$, which is not observed for a similar $\mathrm{La}_{1-x} \mathrm{Sr}_{x} \mathrm{MnO}_{3}$ film grown on $\mathrm{SrTiO}_{3}$ without the $\mathrm{BaTiO}_{3}$ film).

Additional important insights into the electronic and magnetic modifications at manganiteferroelectric interfaces have been gained as well by local atomic characterization using highresolution scanning transmission electron microscopy (HR-STEM), particularly in combination with atomically resolved electron energy loss spectroscopy (EELS), by following the evolution of the energy loss spectra as a function of distance from the interface (although interpretation of such variations in terms of changes in valency is challenging). ${ }^{28,144,971,972}$ In addition, other important information, such as local atomic spacing and oxygen octahedra rotations, can be further related to other properties, such as crystalline structure, oxygen stoichiometry, and ferroelectric polarization; ${ }^{973,974}$ further, by combining STEM and EELS, the specific interface stacking at the interface from one material to the other can be determined. ${ }^{162}$ For example, while XANES measurements demonstrated a change in the valence state in the LSMO/PZT interface, as discussed above, they do not allow one to determine precisely the charge distribution across the interface. ${ }^{315,945}$ Such layer-resolved local measurements of the Mn valence state across ferromagnetic/manganite interfaces using 
Epitaxial ferroelectric interfacial devices

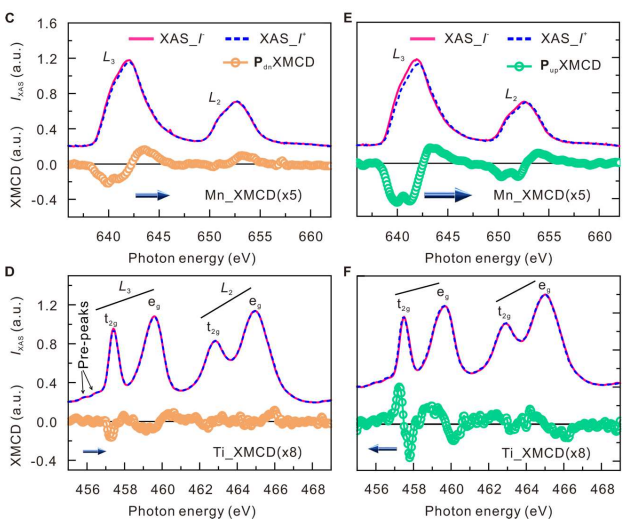

FIG. 20. Normalized X-ray absorption spectroscopy and X-ray magnetic circular dichroism of LSMO/BTO heterostructures at $80 \mathrm{~K}$ and $\pm 1 \mathrm{~T}$ at the Mn (top panels) and Ti (bottom panels) $\mathrm{L}_{2,3}$ edge, for accumulation (left panels) and depletion (right panels) of hole carriers from the LSMO interface. Reproduced with permission from Nano Lett. 19, 3057 (2019). Copyright 2019 American Chemical Society.

STEM-EELS have been reported meanwhile for PZT/LSMO,${ }^{147,156-158}$ BTO/LSMO,${ }^{159,160}$ and $\mathrm{BFO} / \mathrm{CaCeMnO}{ }_{3} \cdot{ }^{161}$ STEM-EELS studies of LSMO/PZT/LSMO,SRO/STO(001) heterostructures, where the two bottom PZT interfaces (LSMO or SRO) are used for setting the direction of the PZT polarization, indicated a drop in Mn valency at the top LSMO/PZT interface layer when the ferroelectric polarization points to that interface (corresponding to depletion of charge carriers), with an opposite behavior when the PZT polarization points in the other direction; changes in the critical temperature and magnetic moment are consistent with the results at 0.2 doping. In a follow up study, ${ }^{147}$ both interfaces of a symmetric $\mathrm{LSMO} / \mathrm{PZT} / \mathrm{LSMO} / \mathrm{STO}(001)$ structure were characterized for one direction of the ferroelectric polarization (although the STEM results show that the interfaces are structurally slightly different, with the top LSMO/PZT interface appearing more diffuse when compared to the atomically sharp bottom interface). The STEM-EELS results suggest a drop in the Mn valency over a distance of about $1 \mathrm{~nm}$ from both PZT interfaces, a surprising result especially for the bottom interface, where an increase in the valence state is expected based on the direction of the ferroelectric polarization $;^{28}$ complementary polar- 
Epitaxial ferroelectric interfacial devices

ized neutron reflectometry measurements carried out to probe the magnetic depth profile of the heterostructure give a much larger magnetic moment also at the bottom interface, a behavior opposite to that found for PZT/LSMO at 0.2 doping ${ }^{315,317,945}$ but consistent with the results for BTO/LSMO/STO(001) at $x=0.3$ doping. ${ }^{956}$ STEM-EELS studies of BTO/LSMO superlattices for several values of the LSMO thickness (4, 10, and 32 u.c.) by Guo et al. ${ }^{159}$ also found a strong decrease in the $\mathrm{Mn}$ valency near both $\mathrm{BaTiO}_{3}$ interfaces. A recent STEM-EELS study of the PZT/12 u.c. LSMO $(x=0.2) / \mathrm{STO}(001)$ interface structure by Meng et al. ${ }^{158}$ has determined the change in the charge transfer across the various interfaces, estimated from the shift in the plasma peaks of valence EELS. The results indicate a strong variation in the valence charge, extending through the whole LSMO layer, $4 \mathrm{~nm}$ into the PZT, and about $2 \mathrm{~nm}$ into the STO substrate interface.

An additional advantage of STEM is the capability of probing the atomic structure in real space and, in particular, of providing important insights into the electronic characteristics of interfaces and of manganite-ferroelectric interfaces. This is important, since several studies have shown that ferroelectric structural distortions can cross the interface between the ferroelectric and the conducting channel and continue for a few atomic layers into the channel region. ${ }^{159,500,501,799,800,951,975}$ For strongly correlated systems, whose physical properties are strongly coupled to the lattice structure, a ferroelectrically induced structural distortion can be expected to induce large modifications of the electronic and magnetic properties. Indeed, a recent STEM study pointed to the important role of changes in the local interfacial atomic structure induced by the polar cations of the ferroelectric in modifying the interfacial orbital occupancy of LSMO via local strain for the accumulation state (see Fig. 16) ${ }^{799}$ while a direct confirmation of the change of the orbital occupancy with the direction of the ferroelectric polarization was confirmed in $\mathrm{La}_{0.8} \mathrm{Sr}_{0.2} \mathrm{MnO}_{3} / \mathrm{PZT}(x=0.175)^{800}$ and $\mathrm{BaTiO}_{3} / \mathrm{La}_{0.8} \mathrm{Sr}_{0.2} \mathrm{MnO}_{3}(x=1 / 3)^{800}$ by measuring the changes in the linear dichroic response of the system at the $\mathrm{Mn} \mathrm{L}$-edge, ascribed to modifications in the interfacial atomic structure that favors occupation of $3 \mathrm{~d} x^{2}-y^{2}$ orbitals for the accumulation state and $3 \mathrm{~d} z^{2}$ orbitals for the depletion state (results that were discussed in section III C in the context of electric field control of the orbital polarization, see Fig. 17).

While ferroelectric field effect control of magnetism in the oxide manganites has received the bulk of the attention, field effects in other oxide systems have also been investigated, including magnetite $\left(\mathrm{Fe}_{3} \mathrm{O}_{4}\right)$ and strontium ruthenate $\left(\mathrm{SrRuO}_{3}\right)$. Magnetite is a conducting 
Epitaxial ferroelectric interfacial devices

mixed valence oxide, where, in an ionic picture, electron transport occurs through electron hopping between $\mathrm{Fe}^{2+}$ and $\mathrm{Fe}^{3+}$ ions on the octahedral sites; since the ions in this sublattice are coupled ferromagnetically, magnetite is expected to be fully spin-polarized. ${ }^{840}$ In principle, as in the doped manganites, changes in the electron charge density should result in changes in the magnetic moment and in the magnetic properties and, effectively, such an effect has been demonstrated via ionic (Li) doping. ${ }^{597-599}$ However, while control of magnetism by interfacing with a ferroelectric has been extensively reported for strainmediated coupling, aiming at taking advantage of the high magnetoelastic coupling coefficients of magnetite ${ }^{976,977}$ electrostatic control of its magnetic properties has been studied less often. ${ }^{978-980}$ For example, Wu et al. ${ }^{979}$ have prepared a $5 \mathrm{~nm}$ thick, [001]-oriented $\mathrm{Fe}_{3} \mathrm{O}_{4}$ film sandwiched between a $200 \mathrm{~nm}$ thick $\mathrm{SrTiO}_{3}$ on top and a bottom $\mathrm{Au} / \mathrm{PZT}$ bilayer, which enabled them to study the electric field response to both strain (via Au/PZT) and electric field (via the $\mathrm{SrTiO}_{3}$ ): they found that, while strain modifies the shape of the hysteresis loop (i.e., the magnetic anisotropy), charge modulation affects mainly the saturation magnetization.

Ferromagnetic strontium ruthenate $\left(\mathrm{SrRuO}_{3}\right)$ is another important material where structural distortions can impact strongly its physical properties. Recent reports have shown that ultrathin $\mathrm{SrRuO}_{3}$ films and $\mathrm{SrRuO}_{3}$-based heterostructures can be a fertile playground for studying novel skyrmion spin structures. Skyrmions are winding spin textures akin to magnetic bubbles ${ }^{981,982}$ but endowed with chirality in systems with broken inversion symmetry by the Dzyaloshinskii-Moriya interaction (DMI). ${ }^{983}$ They have been observed in low symmetry bulk crystals ${ }^{984,985}$ and in asymmetric metallic multilayers between $3 \mathrm{~d}$ transition metal ferromagnetic and heavy metals with large spin-orbit coupling. ${ }^{986-991}$ The latter approach to inducing skyrmion textures has been extended to $\mathrm{SrRuO}_{3},{ }^{992-995}$ a $4 \mathrm{~d}$ transition metal magnetic oxide with an intrinsic spin-orbit coupling of 0.1-0.15 eV ${ }^{996}$ In particular, the work by Wang et al. ${ }^{997}$ showed that, in a $\mathrm{BaTiO}_{3} / \mathrm{SrRuO}_{3}$ heterostructure, a ferroelectrically-driven non-centrosymmetric structural distortion in $\mathrm{SrRuO}_{3}$ layer can trigger a DMI sufficiently large to induce the formation of skyrmion spin structures. Furthermore, they showed that the skyrmion density and stability can be controlled by switching the ferroelectric polarization due to the asymmetric distribution of the latter across the heterostructure. The ability to ferroelectrically control skyrmions in $\mathrm{BaTiO}_{3} / \mathrm{SrRuO}_{3}$ heterostructures opens the possibility to realize novel spintronic devices based on ferroelectric materials. It is also worth 
Epitaxial ferroelectric interfacial devices

noting that the ferroelectrically-driven broken inversion symmetry not only leads to the emergence of skyrmions, but also enhances the ferromagnetic properties of $\mathrm{SrRuO}_{3} .{ }^{992,998}$

\section{Magnetic semiconductors}

Magnetic semiconductors, such as EuO or the dilute magnetic semiconductor (Ga,Mn)As, are characterized by the presence of a band gap at the Fermi level and a relatively low density of thermally activated charge carriers (intrinsic or from dopants). The relevant screening length in these semiconducting systems is the Debye length, which, depending on the carrier density, is typically in the nm range or larger. The origin of magnetism varies with the particular system. In dilute magnetic semiconductors, such as (In,Mn)As, (Ga,Mn)As, and $\mathrm{Mn}_{x} \mathrm{Ge}_{1-x}$, magnetism is driven by the $p$ - $d$ exchange interaction between delocalized hole carriers (from the Mn dopant) and the localized spins of the Mn atoms. ${ }^{999-1006}$ For sufficiently high hole carrier density (of the order of a few \%), a long-range ferromagnetic state develops. In particular, control over the magnetic exchange interaction can be obtained by modulating the carrier density electrostatically, as demonstrated, for example, by gating (In,Mn)As, ${ }^{1007,1008} \mathrm{Mn}_{x} \mathrm{Ge}_{1-x},{ }^{1009-1011}$ (Zn,Mn)Se, ${ }^{1012}$ and (Ga,Mn)As. ${ }^{1013-1019}$ Given the much lower charge carrier density and the large extent of the depletion layer, large modulations of the charge carrier density as provided by ferroelectric gates are not essential and the process is not interfacial in nature, although a high quality interface is still required in order to minimize charge traps. However, the non-volatility provided by a ferroelectric gate could still be of much interest as is the case for ferroelectric FETs.

The reversible control of magnetism has been demonstrated also in the dilute magnetic insulator $\mathrm{TiO}_{2}$ (anatase) doped with Co, ${ }^{1020}$ where both a change in the saturation magnetization and coercive field is observed in $\mathrm{PZT} / \mathrm{Co}: \mathrm{TiO}_{2} / \mathrm{SrRuO}_{3}$ heterostructures as a function of the PZT ferroelectric polarization. Magnetism in the insulator $\mathrm{Co}: \mathrm{TiO}_{2}$ is not mediated by charge carriers, as in the dilute magnetic semiconductors; instead, a bound magnetic polaron percolation picture ${ }^{1021-1023}$ and/or a defect $\left(F\right.$-center) state percolation model ${ }^{1024}$ have been suggested to drive ferromagnetic order in this system. ${ }^{1020}$ 
Epitaxial ferroelectric interfacial devices

\section{FERROELECTRIC TUNNEL JUNCTIONS: FROM BINARY DATA STORAGE TO NEUROMORPHIC COMPUTING}

Ferroelectric tunnel junctions (FTJs) were conceptualized in the early 1970's by Leo Esaki ${ }^{1025}$ although experiments on samples using established ferroelectric films thin enough to act as tunnel barriers were carried out only 30 years later. ${ }^{67}$ The reasons for the time lag include the difficulty to achieve good tunnel barriers (solid-state quantum mechanical tunneling could be achieved early on, for example in superconducting tunnel junctions, ${ }^{1026,1027}$ but magnetic tunnel junctions, for instance, were only developed in the $1990 \mathrm{~s}^{1028}$ ) and to stabilize ferroelectricity in films just a few nm thick (which was only realized in the 2000s). ${ }^{127,466,1029,1030}$ However, Esaki already anticipated that FTJs would exhibit what is now called tunnel electroresistance (TER), a dependence of the tunnel resistance (or current) on the ferroelectric polarization direction, an effect that can be explored for non-volatile memory devices. Importantly, FTJs present an essential advantage over ferroelectric random access memories (FeRAMs): reading the logic state (encoded by the junction's resistance) does not alter the state, resulting in simplified architectures.

The first clear demonstration of TER in FTJs was provided in 2009 by Garcia et al. ${ }^{67}$ Working with ultrathin epitaxial layers of $\mathrm{BaTiO}_{3}$ grown onto $\mathrm{La}_{2 / 3} \mathrm{Sr}_{1 / 3} \mathrm{MnO}_{3}$ bottom electrodes, they used a scanning probe microscope to electrically write micrometer-scale ferroelectric domains with the polarization pointing either up or down, image these domains by piezoresponse force microscopy (PFM), and map the local resistance for both polarization directions. These resistance maps are displayed in Fig. 21(a) for three $\mathrm{BaTiO}_{3}$ barrier thicknesses. While for the lowest thickness $(1 \mathrm{~nm})$ only a weak resistance contrast is visible, at 2 $\mathrm{nm}$ and $3 \mathrm{~nm}$, the resistance shows a strong dependence on the polarization direction, with a contrast reaching $75000 \%$ at room temperature. Importantly, both the tunnel resistance and the TER were found to increase systematically with the barrier thickness, indicating that the $\mathrm{BaTiO}_{3}$ films are good tunnel barriers and that the changes in resistance is linked to the tunneling process.

Three possible physical mechanisms responsible for the TER had been discussed earlier by Tsymbal and Kohlstedt. ${ }^{6}$ One is based on piezoelectricity, causing the barrier thickness to change under the poling voltage and affecting the tunnel resistance; a second mechanism is related to changes of the density of states at the barrier/electrode interfaces; a third 
Epitaxial ferroelectric interfacial devices
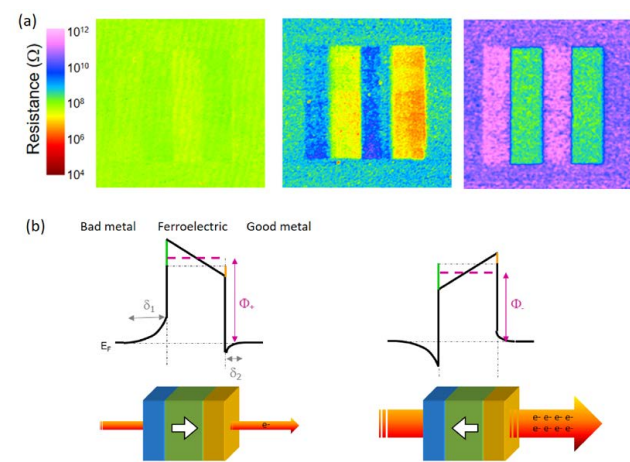

FIG. 21. (a) Conductive AFM resistance maps of BTO/LSMO across $1 \times 4 \mu \mathrm{m}^{2}$ poled domains for (from left to right) 1, 2 and 3 nm-thick BTO barriers. (b) Tunnel barrier potential profiles modified by imperfect screening in the electrodes (good metal on the right side of the barrier with a short screening length $\delta_{2}$; poor metal or semiconductor on the left with a long screening length $\delta_{1}$ ). The dashed purple line shows the average barrier height $\phi_{+}$or $\phi_{-}$for both polarization directions. From reference 67 .

process, which appears to usually dominate the TER response, is electrostatic and was initially proposed by Zhuravlev et al. ${ }^{1031}$ It is described schematically in Fig. 21(b). At the interface between the ferroelectric barrier and the electrodes, polarization charges must be screened; if the electrode is a good metal, with a short screening length ( $\delta_{2}$ in Fig. 21b), the screening is efficient and the electrostatic potential at the interface is only weakly modified; however, if the electrode is a semiconductor or a bad metal with a longer screening length ( $\delta_{1}$ in Fig. 21b), the potential is substantially modified. As a result, in an FTJ combining a good metal and a bad metal sandwiching the tunnel barrier, the barrier potential profile is asymmetric, with an average potential barrier height (purple dashed line in Fig. 21b) that depends on the polarization direction. Because the tunneling current depends exponentially on the square root of the barrier height, switching the ferroelectric polarization results in a large change of the tunnel resistance, producing the TER effect.

In this picture, the TER is thus an interface-driven effect and FTJs qualify as interfacial ferroelectric devices. Among the vast literature on FTJs, ${ }^{1032,1033}$ several studies aimed at addressing these interfacial effects in detail. Two examples are presented in Fig. 22. Figure $22(\mathrm{a}-\mathrm{d})$ shows the resistance at low dc voltage vs the amplitude of short (10 ns) voltage 
Epitaxial ferroelectric interfacial devices

pulses applied to solid-state FTJs based on tetragonal $\mathrm{BiFeO}_{3}$ ferroelectric barriers grown on a bottom electrode of $(\mathrm{Ca}, \mathrm{Ce}) \mathrm{MnO}_{3} \cdot{ }^{1034} \mathrm{~A}$ hysteretic dependence is obtained, reminiscent of the ferroelectric switching (as checked by measuring piezoelectric loops on the same device). The TER amplitude is found to depend strongly on the nature of the metal used as the top electrode, and in fact roughly scaling with the metal work function [Fig. 22(g)]. This reflects the dependence of the TER on the (average) tunnel barrier height, which increases for high work function metals such as Ir. A second example, displayed in Fig. 22(e), shows a similar resistance vs pulse voltage cycle for Pt/BTO/Nb-STO junctions. ${ }^{1035}$ Here, the Nb-doped $\mathrm{SrTiO}_{3}$ bottom electrode has a low carrier density and therefore a large screening length. The amplitude of the TER increases with decreasing Nb doping level, i.e., with increasing the screening length, as shown in Fig. 22(f). As explained above, a strong difference of screening lengths between the top and bottom electrodes should lead to a strong barrier asymmetry and to a larger TER, in agreement with Zhuravlev's model. ${ }^{1031}$

Over the last decade, progress in FTJ research has led to several substantial advances towards device applications. TER values in excess of $10^{8 \%}$ (off/on ratio $>10^{6}$ ), ${ }^{975}$ scaling down to sub $100 \mathrm{~nm}$ sizes ${ }^{1036}$ and compatibility with Si have been demonstrated. ${ }^{1037}$ Significantly, FTJs based on ferroelectric phases based on $\mathrm{HfO}_{2}$ have also been reported, ${ }^{1038-1040}$ paving the way towards the integration of FTJs into CMOS-based processes.

Beyond their possible use as binary non-volatile memory devices, FT.Js have also emerged as interesting candidates for analog-based, neuromorphic computation. Indeed, and as visible in the loops shown in Fig. 22, FTJs can possess intermediate levels of resistance, in addition to the two saturation values. ${ }^{373,1041}$ In fact, there is a quasi-continuum of resistance states between the on and off states that can be stabilized by applying voltage pulses of intermediate amplitudes ${ }^{373,1041}$ or trains of pulses of moderate amplitude. ${ }^{77}$ Importantly, these resistance states are remanent and the resistance of the device only changes when the applied voltage exceeds specific threshold values. These two ingredients qualify FTJs as memristors, ${ }^{1042,1043}$ electronic analogs of the synapses in the brain whose information retention (non-volatility) and plasticity properties are key to the learning process. ${ }^{76}$ The junction conductance plays the role of the synaptic weight in biologic synapses, which is modified as the synapses receive voltage spikes from their neighboring neurons. Compared to biological synapses, FTJs have a higher operation speeds (in the 10-100 ns range versus ms for biological synapses) and very small size (down to nanometer scale for FTJs). These 
Epitaxial ferroelectric interfacial devices
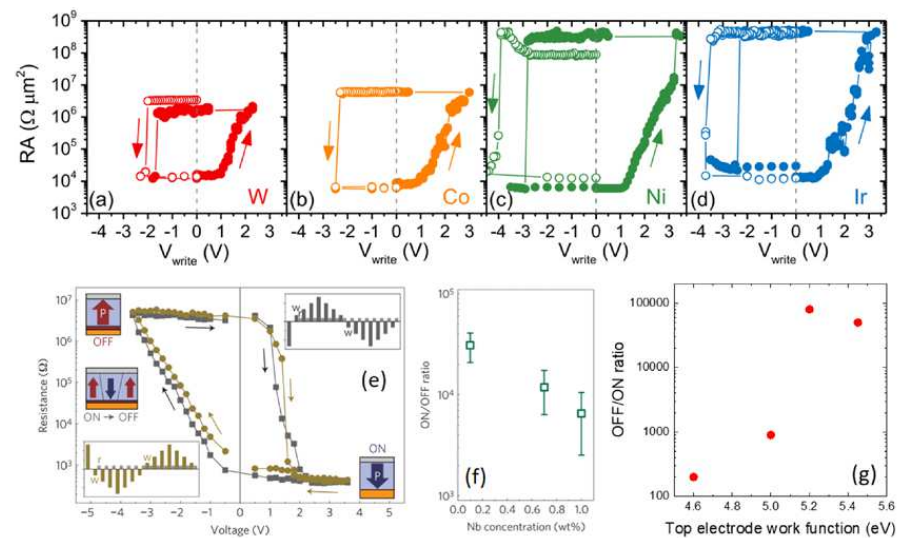

FIG. 22. (a-d) Representative resistance hysteresis cycles for $M / \mathrm{BiFeO}_{3} /(\mathrm{Ca}, \mathrm{Ce}) \mathrm{MnO}_{3}$ junctions with different top electrodes $M=\mathrm{W}, \mathrm{Co}, \mathrm{Ni}$, and $\mathrm{Ir}$, starting from the high resistive state. (e) Resistance hysteresis loops of Pt/BTO/Nb-STO junctions. The loop composed of dark squares (green circles) is obtained by using the writing pulse train ' $\mathrm{w}$ ' between +3.6 and $-3.6 \mathrm{~V}$ shown schematically in the bottom-left (top-right) inset, and measured with read pulses ' $\mathrm{r}$ ' of $+0.1 \mathrm{~V}$ following each write pulse. (f) Off/on ratio in $\mathrm{Pt} / \mathrm{BaTiO}_{3} / \mathrm{Nb}_{-} \mathrm{SrTiO}_{3} \mathrm{FTJ}_{\mathrm{s}}$ for different $\mathrm{Nb}$ concentrations. (g) Off/on ratio as a function of the work function of the metal used as a top electrode in (a-d). (a-d) S. Boyn, V. Garcia, S. Fusil, C. Carrétéro, K. Garcia, S. Xavier, S. Collin, C. Deranlot, M. Bibes, and A. Barthélémy, APL Materials, 3, 061101, 2015; licensed under a Creative Commons Attribution (CC BY) license. (e,f) Reproduced with permission from Nature Materials, 12:617, 2013. Copyright 1998 Macmillan Publishers Limited.

properties allow one to use FTJs to implement specific learning rules observed in biological systems. One is spike-timing dependent plasticity (STDP). ${ }^{76}$ Through STDP, the synaptic weight varies according to the time difference $\Delta t$ between pulses received by the synapses from the pre- and post-synaptic neurons they connect. When the pre-synaptic neuronal spike reaches the synapse before the post-synaptic neuronal spike $(\Delta t>0)$, the synaptic weight typically increases, while it decreases for $\Delta t<0$. STDP can be implemented in memristors by mimicking the neuronal spikes by voltage waveforms having specific shapes. In the example of Fig. 23(a), one voltage waveform is comprised of a rectangular positive wavefront and a negative trapezoidal long slope. Within a single pulse the voltage never 
Epitaxial ferroelectric interfacial devices

exceeds the memristor's threshold for switching. When two waveforms are combined to mimic the reception of neuronal spikes coming from the pre-synaptic and the post-synaptic neurons, they add up so that if $|\Delta t|>0$ is sufficiently short, the threshold voltage will be exceeded and the memristor conductance will change (increasing or decreasing depending on the sign of $\Delta t$ ). However, if $\Delta t$ is long, the waveforms do not overlap, the switching threshold is not reached and the conductance remains unchanged.

Figures 23(a,b) show examples of the realization of STDP in FTJs based on $\mathrm{BiFeO}_{3}{ }^{77}$ and $\mathrm{Hf}_{0.5} \mathrm{Zr}_{0.5} \mathrm{O}_{2}(\mathrm{HZO}),{ }^{78}$ respectively. In both cases a clear dependence of the junction conductance with $\Delta t$ is observed. Remarkably, STDP in such FTJs can be harnessed to perform pattern recognition. This is illustrated in Fig. 23(c,d) for a $6 \times 6$ FTJ array used to encode the character ' $F$ '. The array is trained by sending sequences of half bias voltage pulses, i.e., half the voltage needed to switch a cell, so that only cells at the intersection (fully selected cells) between lines and rows are actually switched, corresponding to the pattern to be learned. While initially all FTJs are in a low conductance state, after the first sequence the conductance of the fully selected cells is clearly updated, as visible in the $6 \times 6$ conductance map displayed in Fig. 23(c). After subsequent training sequences, the conductance contrast between these cells and the others increases, although some errors can occur in the process (see 2nd training). However, after 10 training cycles, the conductance maps accurately reflect the expected pattern: the character ' $\mathrm{F}$ ' has been learned by the array. Figure 23(d) shows the conductance maps of patterns 'F', 'D' and 'U' after training for 10,25 and 38 cycles, respectively.

\section{FERROELECTRIC SPINTRONICS INTERFACIAL DEVICES}

Ferroelectrics can accumulate or deplete charge at the interface with an adjacent material, while polar atomic displacements at such interfaces can alter the atomic distances and orbital overlap in the adjacent material. Both mechanisms may lead to polarization direction dependent modifications of the local density of states. If the adjacent material is a ferromagnet with a spin-split density of states, these modifications need not be the same for the spin up and spin down states and therefore switching the ferroelectric will yield a change of the interfacial spin-polarization $P_{\sigma}$ (the normalized difference between the density of states for spin up and spin down states at the Fermi energy). Such a depen- 
Epitaxial ferroelectric interfacial devices
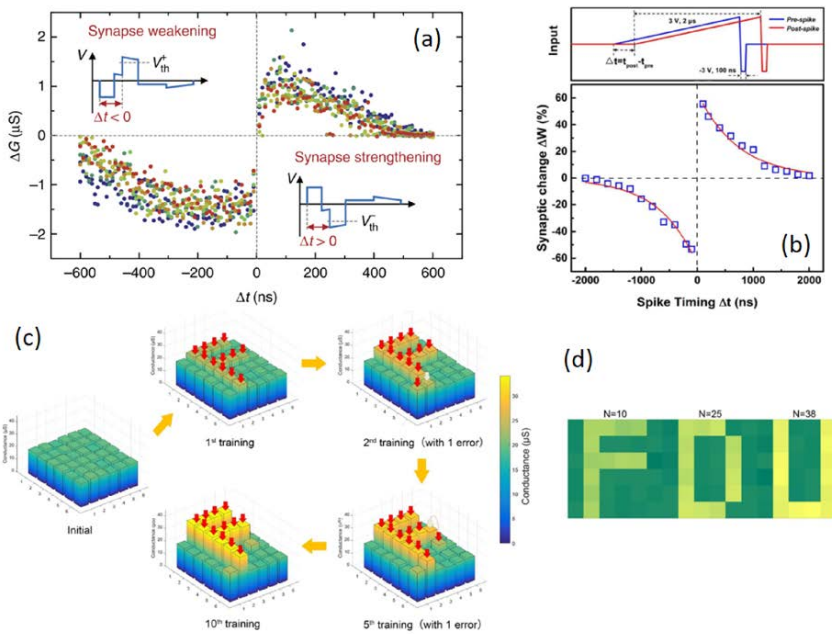

(d)

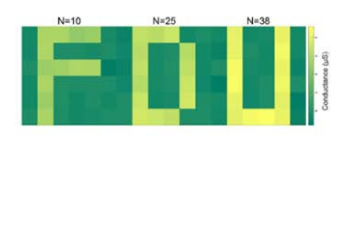

FIG. 23. (a) Measurements of STDP in a $\mathrm{Co} / \mathrm{BTO} /(\mathrm{Ca}, \mathrm{Ce}) \mathrm{MnO}_{3}$ ferroelectric memristor. Modulation of the device conductance $(\Delta G)$ as a function of the delay $(\Delta t)$ between pre- and post-synaptic spikes. Seven data sets were collected on the same device showing the reproducibility of the effect. The total length of each pre- and post-synaptic spike is $600 \mathrm{~ns}^{77}$ (b) STDP measurements in a HZO-based FTJ. The top panel shows the shape of the pre- and post-synaptic spikes. The red lines are exponential fits to the data. (c) Training evolution of pattern ' $\mathrm{F}$ ' in the $6 \times 6$ HZO-based FTJs array. Random false training pulses were involved during the 2nd and 5th training steps to verify its fault tolerance. (d) Conductance maps of patterns ' $\mathrm{F}$ ', 'D' and ' $U$ ' after training for 10,25 and 38 cycles, respectively, and the corresponding statistical distribution of synapse conductance. (a) S. Boyn, J. Grollier, G. Lecerf, B. Xu, N. Locatelli, S. Fusil1, S. Girod, C. Carrétéro, K. Garcia, S. Xavier, J. Tomas, L. Bellaiche, M. Bibes, A. Barthélémy, S. Saïghi, V. Garcia, Nature Communications, 8, 14736, 2017; licensed under a Creative Commons Attribution (CC BY) license. (b-d) Adapted with permission from Nanoscale 10, 15826 (2018). Copyright 2018 The Royal Society of Chemistry.

dence can be harnessed in spintronics devices, whose response usually directly depends on the spin polarization. This is the case for magnetic tunnel junctions in which the tunnel magnetoresistance (TMR) is directly linked to the spin polarization value through Jullière's formula ${ }^{1044} \mathrm{TMR}=2 P_{\sigma 1} P_{\sigma 2} /\left(1-P_{\sigma 1} P_{\sigma 2}\right)$ where $P_{\sigma 1}$ and $P_{\sigma 2}$ are the spin polarizations 
Epitaxial ferroelectric interfacial devices

of the two ferromagnetic electrodes interfacing the barrier. Hence, changing $P_{\sigma}$ by ferroelectric switching should yield a change in the TMR value, through an effect coined tunnel electromagnetoresistance (TEMR). ${ }^{1045}$
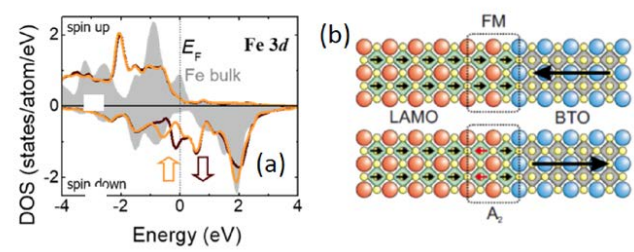

FIG. 24. (a) Majority (top) and minority (bottom) spin density of states of $\mathrm{Fe}$ at the $\mathrm{Fe} / \mathrm{BaTiO}_{3}$ interface for two opposite ferroelectric polarization directions. The gray shaded plot is the density of states away from the interface. (b) Sketch of the electrically induced magnetic reconstruction at the interface $\mathrm{La}_{0.5} A_{0.5} \mathrm{MnO}_{3} / \mathrm{BaTiO}_{3}(A=\mathrm{Ca}$, $\mathrm{Sr}$, or $\mathrm{Ba})$. The arrangement of magnetic moments at Mn sites (small arrows) changes from ferromagnetic (FM) to antiferromagnetic type A when the ferroelectric bias (large arrows) of the $\mathrm{BaTiO}_{3}$ is reversed. (a) Adapted with permission from Phys. Rev. Lett. 97, 047201 (2006). Copyright 2006 by the American Physical Society. (b) Reproduced with permission from Phys. Rev. B 80, 174406 (2009). Copyright 2009 by the American Physical Society.

Focusing mostly on $\mathrm{Fe} / \mathrm{BaTiO}_{3}$ interfaces, several groups predicted changes in the amplitude of the magnetic moments carried by $\mathrm{Fe}$ and $\mathrm{Ti}$ and of the interfacial spin polarization, depending on the ferroelectric polarization direction, as shown in Fig. 24(a). ${ }^{909,910}$ To probe these effects experimentally, Garcia et al. fabricated $\mathrm{Fe} / \mathrm{BaTiO} / \mathrm{LSMO}$ nanoscale tunnel junctions [Fig.25(a)]. ${ }^{1045}$ The junctions showed negative TMR at $4 \mathrm{~K}$, indicating that the $\mathrm{Fe} / \mathrm{BaTiO}_{3}$ interface tunnel spin-polarization is negative, consistent with ab initio calculations. ${ }^{909,910,913}$ Switching the ferroelectric polarization of the $\mathrm{BaTiO}_{3}$ barrier resulted in changes of the negative TMR: the TMR was large (small) when the ferroelectric polarization points towards the Fe (LSMO) layer. ${ }^{1045}$ The relative variation of the TMR with the ferroelectric polarization corresponds to a TEMR effect of up to $450 \%$.

These results were then extended to $\mathrm{Co} / \mathrm{BaTiO}_{3} / \mathrm{LSMO}$ junctions, with comparable TEMR effects. ${ }^{916}$ Pantel et al. ${ }^{1046}$ subsequently showed that it is even possible to reverse the sign of the TMR by switching the ferroelectric polarization in Co/PZT/LSMO junc- 
Epitaxial ferroelectric interfacial devices

tions [Fig.25(b)]. In these experiments, the ferroelectric polarization direction in the PZT tunnel barrier controls the sign of the current spin-polarization, providing new pathways for electrically controlled spintronic devices.

Specific interface engineering can also yield interesting and large TEMR effects. Yin et al. ${ }^{1047}$ introduced an interfacial $\mathrm{La}_{0.5} \mathrm{Ca}_{0.5} \mathrm{MnO}_{3}$ layer in $\mathrm{LSMO} / \mathrm{BaTiO}_{3} / \mathrm{LSMO}$ junctions and observed large modifications of the TMR. As illustrated in Fig. 24(b), the polarizationinduced metal/insulator phase transition in $\mathrm{La}_{0.5} \mathrm{Ca}_{0.5} \mathrm{MnO}_{3}$ is accompanied by a transition between ferromagnetic and antiferromagnetic states. At $80 \mathrm{~K}$, the TMR is about $100 \%$ when the ferroelectric polarization points towards $\mathrm{La}_{0.5} \mathrm{Ca}_{0.5} \mathrm{MnO}_{3}$, corresponding to its ferromagnetic state, but is close to zero when pointing in the opposite direction, corresponding to an antiferromagnetic state. Driving an interfacial magnetic phase transition by switching the ferroelectric polarization of the tunnel barrier thus appears to be an efficient way to control the spin-polarization of the tunnel current (with a TEMR reaching $500 \%$ in these devices).
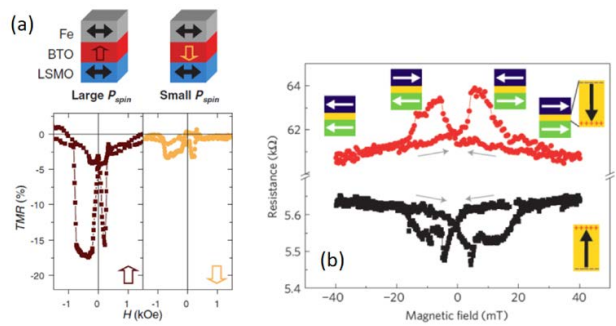

FIG. 25. (a) TMR curves of a $\mathrm{Fe} / \mathrm{BaTiO}_{3} / \mathrm{LSMO}$ tunnel junction measured at electrical remanence and $4 \mathrm{~K}$, after switching the ferroelectric polarization up or down. The large variation of the TMR reflects changes in the interfacial Fe spin polarization due to ferroelectric polarization reversal. (b) TMR at $50 \mathrm{~K}$ in the as-grown state of a Co/PZT/LSMO junction (black squares) and after polarization switching (red circles). The ferroelectric polarization states of the barrier as well as the magnetization directions in the electrodes are shown by arrows. (a) From Science 327, 1106 (2010). Reprinted with permission from AAAS. (b) Reproduced with permission from Nature Materials, 11, 289 (2012). Copyright 2012 Macmillan Publishers Limited.

Recently, new ideas have arisen that take advantage of ferroelectrically controlled interfacial effects for spintronics in the context of the renewed interest in Rashba spin-orbit coupling (RSOC). ${ }^{1048,1049}$ RSOC typically occurs at surfaces and interfaces where spatial 
Epitaxial ferroelectric interfacial devices

symmetry breaking results in a built-in electric potential along the out-of-plane direction $\mathbf{z}$. The corresponding Rashba Hamiltonian can be expressed as $H_{R}=\alpha_{R}(\mathbf{k} \times \boldsymbol{\sigma}) \cdot \mathbf{z}$, where $\boldsymbol{\sigma}$ is the vector of the Pauli spin matrices, $\mathbf{k}$ the momentum and $\alpha_{R}$ the Rashba coefficient, which is proportional to the electric field strength and the spin-orbit interaction. In a Rashba system the momentum and spin degrees of freedom are locked, as illustrated in Fig. 26(a-c) and the spin degeneracy of the 2D band structure is lifted.

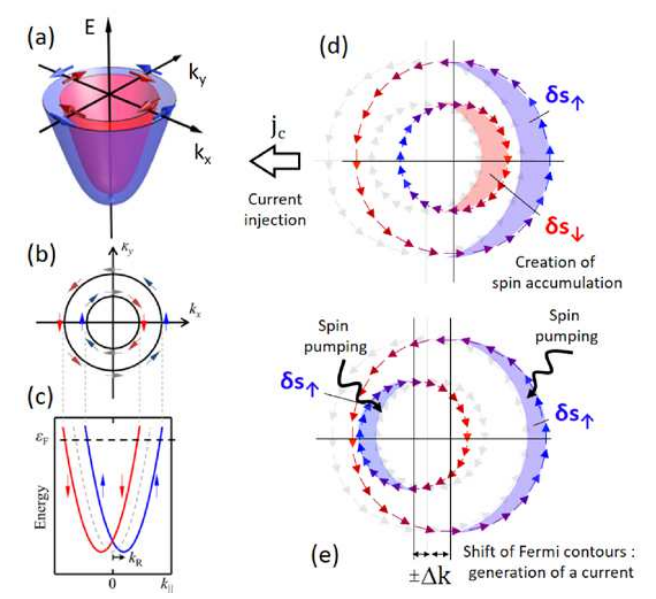

FIG. 26. (a) The momentum-dependent spin split sub-bands in a Rashba system. (b) Energy cut showing the two Fermi contours with chiral and opposite spin textures. (c) Rashba split energy bands. (d) Direct Edelstein effect: a charge current $j_{c}$ injected along $-x$ induces a shift $\Delta k$ of both Fermi contours, resulting in a spin accumulation $\delta_{s \uparrow}-\delta_{s \downarrow}$ polarized along the $y$ axis due to the inequivalence of the two contours. (e) Inverse Edelstein effect: when a spin current is injected (e.g by spin pumping) with the spin polarization along the $y$ axis, the spin population is altered in the steady state causing a displacement in momentum space of the two inequivalent Fermi surfaces (red and blue lines) by $\pm \Delta k$. This results in a net charge current generated perpendicular to the spin current and to its spin polarization.

RSOC is appealing for spintronics because it allows one to interconvert spin and charge currents and represents a promising pathway towards ferromagnet-free spintronics (by using RSOC systems as sources and detectors of spin currents, tasks conventionally performed with ferromagnets). ${ }^{1050}$ When a charge current flows in a Rashba system, it can generate 
Epitaxial ferroelectric interfacial devices

a transverse spin accumulation; this effect is called the Edelstein (or Rashba-Edelstein) effect, ${ }^{1051}$ as illustrated in Fig. 26(d). This spin accumulation can diffuse into an adjacent conducting material through the interface and generate a pure 3D spin current without net charge current (that is, a pure flow of spin angular momentum, with opposite flows for electrons of opposite spins). Conversely, the injection of a spin current in a Rashba system generates a net charge current (inverse Edelstein effect, IEE), ${ }^{1052}$ see Fig. 26(e). The efficiency of the IEE can be estimated by the figure of merit $\lambda_{\text {IEE }}$ given by the ratio between the $2 \mathrm{D}$ charge current generated, $j_{c}^{2 \mathrm{D}}$, and the $3 \mathrm{D}$ spin current injected, $j_{s}^{3 \mathrm{D}}$. Note that since one current is $2 \mathrm{D}$ and the other is $3 \mathrm{D}, \lambda_{\mathrm{IEE}}$ has the dimension of length. In the simplest case of circular Fermi contours, $\lambda_{\text {IEE }}$ is proportional to the Rashba coefficient $\alpha_{R}$ and the relaxation time $\tau$, so that $\lambda_{\mathrm{IEE}}=\left(\alpha_{R} \tau\right) / \hbar$. One way to optimize the spinto-charge conversion is therefore to find systems with substantial Rashba SOC and long relaxation times. Interestingly, bulk materials with broken inversion symmetry may also exhibit a Rashba effect, three-dimensional in nature (3D Rashba), as was first identified in BiTeI. ${ }^{1053}$ The polar nature of ferroelectrics makes them prime candidates to harbor bulk RSOC, with the bonus that it could also be switchable by an electric field. The last few years have seen efforts towards the identification of single-phase Rashba ferroelectrics, ${ }^{1054}$ mostly focusing on GeTe, a low bandgap ferroelectric semiconductor with a $T_{\mathrm{C}}$ of $700 \mathrm{~K}$. GeTe has been predicted to be a bulk Rashba material, ${ }^{1055}$ where the ferroelectric polarization switching causes a full reversal of the spin texture of the Rashba split Fermi contours. Until recently, because of high leakage, indications of ferroelectricity have only been provided in thin films using piezoresponse force microscopy (PFM), ${ }^{1056}$ but recent results show a strong electroresistance effect reflecting polarization switching. ${ }^{1057}$ The surface band structure of GeTe has been mapped by angle resolved photoemission spectroscopy (ARPES), revealing a strong Rashba splitting that depends on the ferroelectric polarization state (in two different samples, ${ }^{1058}$ or on the same in situ poled sample ${ }^{1059}$ ). Very recent results have demonstrated a ferroelectric control of spin-charge converrsion with GeTe, at room temperature. ${ }^{1057} \mathrm{In}$ parallel, a switchable Rashba SOC has been predicted in more classical compounds from the perovskite family including $\mathrm{BiAlO}_{3}{ }^{1060}$, strained $\mathrm{KTaO}_{3},{ }^{1061}$ strained $\mathrm{SrBiO}_{3},{ }^{1062}$ as well as in other oxide compounds such as Aurivilius phases. ${ }^{1063}$

Charge accumulation/depletion and polarization-dependent atomic reconstruction at ferroelectric interfaces also offer opportunities to control interfacial RSOC by electric fields, in 
Epitaxial ferroelectric interfacial devices

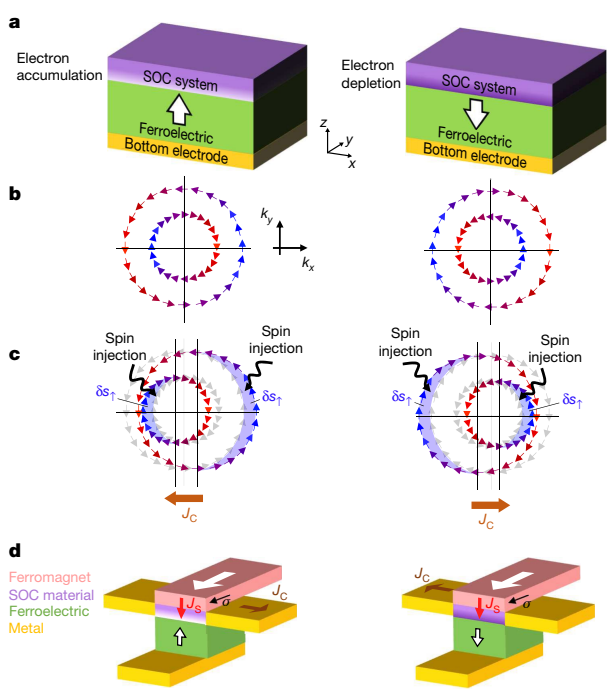

FIG. 27. (a) Sketch of a ferroelectric Rashba architecture combining a ferroelectric material (green) and a material with spin-orbit coupling (purple). Upon switching the polarization, electrons accumulate (left) or deplete (right) in the SOC material, creating an electric field whose sign depends on the polarization direction. (b) Corresponding Rashba-split chiral Fermi contours with spin-momentum locking. The chirality of the contours switches upon switching ferroelectric polarization. (c) Inverse Edelstein effect in a Rashba interface. When a spin current is injected (e.g. by spin pumping) with a spin polarization along the $y$ axis, the spin population is altered, causing a displacement in momentum space of the two inequivalent Fermi surfaces (red and blue lines) by $\pm \Delta k$. This results in a net charge current, generated perpendicular to the spin current and to its spin polarization. The sign of the generated current depends on the chirality of the Fermi contours and is therefore reversed upon switching the ferroelectric polarization. (d) Non-volatile device operated by ferroelectricity and Rashba SOC. A charge current $J_{C}$ is generated by the conversion of a spin current $J_{S}$ through the inverse Edelstein effect. The sign of $J_{C}$ changes with the direction of the ferroelectric polarization. From reference 1064. Copyright 2020, The Authors, under exclusive licence to Springer Nature Limited.

a non-volatile way [cf. Fig. 27(a-c)]. The first prediction of ferroelectric control of Rashba SOC was by Mirhosseini et al. ${ }^{1065}$ in $\mathrm{Bi} / \mathrm{BaTiO}_{3}$. The dependence of the Rashba coefficient 
Epitaxial ferroelectric interfacial devices

was modest, but Zhong et al. ${ }^{1066}$ later calculated a large (0.1-0.7 eVA) and fully switchable Rashba coefficient in various types of perovskite interfaces combining $\mathrm{BaTiO}_{3}$ with $\mathrm{BaRuO}_{3}, \mathrm{BaIrO}_{3}$ or $\mathrm{BaOsO}_{3}$. Recently, Song et al. ${ }^{1067}$ predicted a large effect as well in $\mathrm{PbTiO}_{3} / \mathrm{BiInO}_{3}$ interfaces, see Fig. 28.

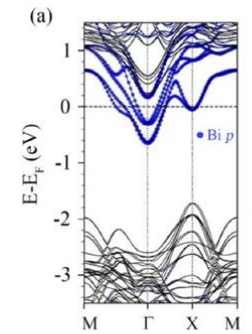

(c)

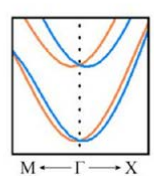

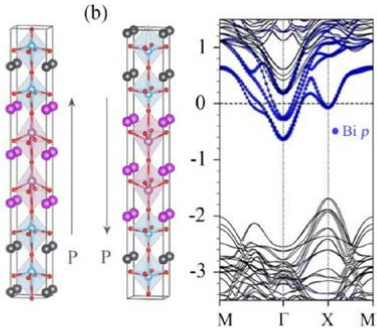

(d)

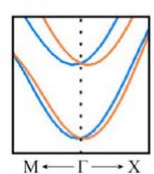

FIG. 28. Band structure of symmetric $\mathrm{PbTiO}_{3} / \mathrm{BiInO}_{3}$ heterostructure with ferroelectric polarization along (a) [001] and (b) [001] . The blue circles represent Bi $p$ orbitals. Rashba bands around the $\Gamma$ point below the Fermi level are magnified in (c) and (d). Y. Song, D. Zhang, B. Xu, K. Chang, and C.W. Nan. ArXiv, 1909.03727, 2019; licensed under a Creative Commons Attribution (CC BY) license.

Two-dimensional electron gases (2DEGs) based on $\mathrm{SrTiO}_{3}{ }^{701}$ are known to display a sizeable $\mathrm{RSOC}^{670}$ that has been harnessed to achieve very efficient spin-charge $\mathrm{e}^{1068,1069}$ and charge-spin conversion. ${ }^{1070,1071}$ Taking advantage of the possibility to induce a ferroelectriclike state in $\mathrm{SrTiO}_{3}$ by applying large electric fields, ${ }^{1072}$ Noöl et al. have demonstrated a non-volatile electrical control of giant spin-charge conversion in $\mathrm{SrTiO}_{3}$ 2DEGs. ${ }^{1064}$

Achieving ferroelectric control of RSOC offers many types of device opportunities for spintronics, one being illustrated in Fig. 27(d). In this device, a ferromagnet with a fixed magnetization direction is used to inject a spin current into a Rashba state at the interface between a ferroelectric and a material containing a heavy element (e.g. the $\mathrm{PbTiO}_{3} / \mathrm{BiInO}_{3}$ interface mentioned above). ${ }^{1067}$ The spin current injection proceeds by tunneling across the ferroelectric (as in the FTJs described in section IV), which must be sufficiently thin. 
Epitaxial ferroelectric interfacial devices

Depending on the ferroelectric polarization direction, the chirality of the spin contours in the Rashba state will switch, resulting in a change in sign of the effective Rashba coefficient and a reversal in sign of the produced charge current. This illustrates how ferroelectricity could control the inverse Edelstein effect. Reciprocally, the direct Edelstein effect could be controlled ferroelectrically in a similar way, leading to the generation of spin currents with up or down spin polarization from a given charge current of fixed sign and used to manipulate spin textures in a neighboring layer.

\section{CONCLUSIONS AND OUTLOOK}

In this overview of the recent developments of ferroelectric interfacial phenomena and devices, we covered both the basic aspects of thin film ferroelectricity and the several device structures where the ferroelectric polarization has been employed to control the interfacial charge carrier density to modulate the correlated state of matter, or the charge tunneling transport. We highlighted the recent progress achieved in the growth of high quality, single-crystalline ferroelectric thin films and in the fundamental understanding of the factors defining and limiting the ferroelectric state in the ultrathin limit. In particular, we emphasized the importance of the elastic and electric boundary conditions in determining the ferroelectric properties of thin films, exemplified for the canonical ferroelectric perovskite systems $\mathrm{BaTiO}_{3}, \mathrm{PbTiO}_{3}, \mathrm{~Pb}\left(\mathrm{Zr}_{0.2} \mathrm{Ti}_{0.8}\right) \mathrm{O}_{3}, \mathrm{BiFeO}_{3}$, and a more recent addition to the ferroelectric family, $\mathrm{HfO}_{2}$, with its proven compatibility with Si CMOS, high band gap and high dielectric breakdown voltage, making it a promising materials system for device applications. While we have described only a subset of the large family of ferroelectric systems, our choice of systems illustrate the various phenomena that typically occur at the ultrathin film limit, including the role of epitaxial strain in modifying the ferroelectric properties (and in some cases of stabilizing supertetragonal phases with very high ferroelectric polarizations, which are of high interest for device structures), and of the electrical boundary conditions, since the relatively small band gap of most ferroelectrics makes consideration of band alignment and Schottky barriers critical for device function. Although we considered here only the basic aspects associated with the static band alignment, it is clear that a working device must fulfill the conditions that allow for switching the ferroelectric polarization, including high endurance and stability, which depend in large measure on the presence and evolution 
Epitaxial ferroelectric interfacial devices

of defects present in the ferroelectric film and largely formed during the growth process, which we also discussed in detail. Indeed, although much progress has been achieved, certain aspects still require improvement; for example, imprint effects, where built-in electric fields lead to the favouring of one direction of the ferroelectric polarization lead to time instability of the ferroelectric state and depolarization; in a worst case scenario, the opposite polarization state may not be reached at remanence, resulting in reduced field effects. ${ }^{507}$

Importantly, in the context of ferroelectric interfacial devices, ferroelectricity has now been demonstrated in ferroelectric films a few unit cells in thickness, settling a long controversy over the intrinsic or extrinsic nature of the so-called ferroelectric dead layer deduced in early reports of ferroelectricity in thin films, and paving the way for the realization of ferroelectric tunneling devices where very large changes in the conduction state are achieved as a function of the direction of the ferroelectric polarization, and of low voltage switching, high polarization ferroelectric field effect device structures.

In discussing ferroelectric interfacial phenomena, we have largely focused on controlling the correlated state in complex oxide materials using oxide ferroelectric systems. One advantage of such a materials combination is the compatibility as far as the oxide growth process is concerned, the stability of oxides to ambient conditions and the wide range of physical properties that have been identified in such materials systems. Specifically, we have reviewed the control of superconductivity and of the insulator to metal transition, where field effect control of the conducting state aimed at achieving devices with high on/off resistance ratios and lower switching energies as an alternative to CMOS switches. Although in some instances large changes in the resistivity ratio have been obtained, a full control over the order parameter (wave function amplitude in the case of superconductivity, translation symmetry for the metal insulator transition) has proved challenging thus far. Electric field control of orbital and charge order has also been achieved in some instances, although the difficulty in these cases lies in probing the changes in the ordered state, since they require probes that are sensitive to the orbital or valence state. In comparison, we argue that more rapid progress has been achieved in controlling the interfacial magnetic state with electric fields, given the many probes available for measuring magnetic moments and the clearer physical processes linking spin order to charge (even if the details of such processes may be complex in nature). The most clear example is the case of the manganite perovskites, where the dependence of the magnetic properties with charge are known from the bulk phase diagrams, but where the 
Epitaxial ferroelectric interfacial devices

presence of epitaxial strain in thin films and bonding effects at the interface result in more complex and richer magnetic and electronic behavior. Unveiling the details of the coupling mechanism between charge and spin order and a better understanding of how that process evolves at the different magnetic phase boundaries across the large doped manganite family still remains to be carried out.

Also impressive has been the rapid development of ferroelectric tunnel junctions, from the theoretical and experimental demonstration to applications in device structures, which we briefly reviewed here. Given the challenge of demonstrating ferroelectricity in such ultrathin ferroelectric tunnel barriers, this endeavor has required a painstaking process of ensuring that the required properties are present at the various levels of the device structure. For example, given the large tunneling current across such structures, observation of the displacement associated with the ferroelectric switching is not possible, while the large electric field applied to such ferroelectric barriers possibly suggests that a large fraction of the applied electric field drops at interfacial dielectric layers within the structure.

Clearly, the approaches reviewed here for oxide materials can in principle be extended for other family of correlated systems and ferroelectric materials. Critical are good quality interfaces, good ferroelectric properties down to the ultrathin limit, and correlated systems whose properties show a large sensitivity to the charge density. The current progress in this area shows that controlling the correlated state of matter at ferroelectric interfaces provides not only a means to better understand at a fundamental level the role of electron interactions in determining the electronic properties of materials, but also as a mean of achieving new functionalities that may be harnessed for electronic devices capable of complex functions, such as low power logic switches, complex logic operations, nanometer scale logic memories, and neuron-like memory elements.

\section{ACKNOWLEDGMENTS}

We are very thankful to the authors that kindly allowed reproduction of their work here. C.A.F.V. acknowledges support from the Swiss National Science Foundation grant number 200021_184684. C.H.A., Y.S. and F.J.W. acknowledge support from ONR grant number N000144-19-1-2104. M.B. acknowledges support from the ERC Advanced Grant number 833973 "FRESCO". K.M.R. acknowledges support from ONR grant N000144-19-1-2073. 
Epitaxial ferroelectric interfacial devices

\section{DATA AVAILABILITY}

Data sharing is not applicable to this article as no new data were created or analyzed in this study.

\section{REFERENCES}

${ }^{1}$ P. Zubko, S. Gariglio, M. Gabay, P. Ghosez, and J.-M. Triscone. Ann. Rev. Condens. Matter Phys., 2:141, 2011.

${ }^{2}$ C. A. F. Vaz, F. J. Walker, C. H. Ahn, and S. S. Ismail-Beigi. J. Phys.: Condens. Matter, 27:123001, 2015.

${ }^{3}$ C. H. Ahn, J.-M. Triscone, and J. Mannhart. Nature, 424:1015, 2003.

${ }^{4}$ C. H. Ahn, A. Bhattacharya, M. Di Ventra, J. N. Eckstein, C. D. Frisbie, M. E. Gershenson, A. M. Goldman, I. H. Inoue, J. Mannhart, A. J. Millis, A. F. Morpurgo, D. Natelson, and J.-M. Triscone. Rev. Mod. Phys., 78:1185, 2006.

${ }^{5}$ A. M. Goldman. Annu. Rev. Mater. Res., 44:45, 2014.

${ }^{6}$ E. Y. Tsymbal and H. Kohlstedt. Science, 313:181, 2006.

${ }^{7}$ E. Y. Tsymbal, A. Gruverman, V. Garcia, M. Bibes, and A. Barthélémy. MRS Bull., 37:138, 2012.

${ }^{8}$ M. Dawber, K. M. Rabe, and J. F. Scott. Rev. Mod. Phys., 77:1083, 2005.

${ }^{9} \mathrm{Ph}$. Ghosez and J. Junquera. First-principles modeling of ferroelectric oxide nanostructures. In M. Rieth and W. Schommers, editors, Handbook of theoretical and computational nanotechnology, page 623. American Scientific Publisher, Stevenson Ranch, CA, 2006. Preprint: arXiv:cond-mat/0605299v1 [cond-mat.mtrl-sci].

${ }^{10}$ N. Setter, D. Damjanovic, L. Eng, G. Fox, S. Gevorgian, S. Hong, A. Kingon, H. Kohlstedt, N. Y. Park, G. B. Stephenson, I. Stolitchnov, A. K. Taganstev, D. V. Taylor, T. Yamada, and S. Streiffer. J. Appl. Phys., 100:051606, 2006.

${ }^{11}$ I. A. Kornev, H. Fu, and L. Bellaiche. J. Materials Science, 41:137, 2006.

${ }^{12}$ D. G. Schlom, L.-Q. Chen, C.-B. Eom, K. M. Rabe, S. K. Streiffer, and J.-M. Triscone. Annu. Rev. Mater. Res., 37:589, 2007.

${ }^{13}$ Y. Wang, W. Chen, B. Wang, and Y. Zheng. Materials, 7:6377, 2014.

${ }^{14}$ L. W. Martin and A. M. Rappe. Nature Reviews, 2:16087, 2017. 
Epitaxial ferroelectric interfacial devices

${ }^{15}$ E. Bousquet, M. Dawber, N. Stucki, C. Lichtensteiger, P. Hermet, S. Gariglio, J.-M. Triscone, and P. Ghosez. Nature, 452:732, 2008.

${ }^{16}$ M. Dawber, N. Stucki, C. Lichtensteiger, S. Gariglio, and J.-M. Triscone. J. Phys.: Condens. Matter, 20:264015, 2008.

${ }^{17}$ M. Finazzi, L. Duò, and F. Ciccacci. Surface Science Reports, 64:139, 2009.

${ }^{18}$ J. Mannhart and D. G. Schlom. Science, 327:1607, 2010.

${ }^{19}$ L. F. Kourkoutis, H.L. Xin, T. Higuchi, Y. Hotta, J. H. Lee, Y. Hikita, D. G. Schlom, H. Y. Hwang, and D. A. Muller. Phil. Mag., 90:4731, 2010.

${ }^{20}$ M. Bibes, J. E. Villegas, and A. Barthélémy. Adv. Phys., 60:5, 2011.

${ }^{21}$ M. Opel, S. Geprägs, E. P. Menzel, A. Nielsen, D. Reisinger, K.-W. Nielsen, A. Brandlmaier, F. D. Czeschka, M. Althammer, M. Weiler, S. T. B. Goennenwein, J. Simon, M. Svete, W. Yu, S.-M. Hühne, W. Mader, and R. Gross. Phys. Status Solidi A, 208:232, 2011.

${ }^{22}$ J. M. Rondinelli and N. A. Spaldin. Adv. Mater., 23:3363, 2011.

${ }^{23}$ H. Y. Hwang, Y. Iwasa, M. Kawasaki, B. Keimer, N. Nagaosa, and Y. Tokura. Nature Materials, 11:103, 2012.

${ }^{24}$ J. Chakhalian, A. J. Millis, and J. Rondinelli. Nature Materials, 11:92, 2012.

${ }^{25}$ M. Fiebig. Phil. Trans. R. Soc. A, 370:4972, 2012.

${ }^{26}$ J. M. Rondinelli, S. J. May, and J. W. Freeland. MRS Bulletin, 37:261, 2012.

${ }^{27}$ J. Chakhalian, J. W. Freeland, A. J. Millis, C. Panagopoulos, and J. M. Rondinelli. Rev. Mod. Phys., 86:1189, 2014.

${ }^{28}$ H. Guo, M. Saghayezhian, Z. Wang, Y. Zhu, J. Zhang, and W. Plummer. Front. Phys., $15: 13401,2020$.

${ }^{29}$ M. E. Lines and A. M. Glass. Principles and applications of ferroelectrics and related materials. Clarendon Press, Oxford, 1977.

${ }^{30}$ K. M. Rabe, M. Dawber, C. Lichtensteiger, C. H. Ahn, and J.-M. Triscone. Modern physics of ferroelectrics: essential background. In K. Rabe, C. H. Ahn, and J.-M. Triscone, editors, Physics of ferroelectrics, volume 105 of Topics in Applied Physics, page 1. Springer-Verlag, Berlin, 2007.

${ }^{31}$ R. E. Cohen. J. Phys. Chem. Solids, 61:139, 2000.

${ }^{32}$ N. A. Hill. J. Phys. Chem. B, 104:6694, 2000.

${ }^{33}$ D. I. Khomskii. J. Magn. Magn. Mater., 306:1, 2006. 
Epitaxial ferroelectric interfacial devices

${ }^{34}$ K. M. Rabe and P. Ghosez. First-principles studies of ferroelectric oxides. In K. Rabe, C. H. Ahn, and J.-M. Triscone, editors, Physics of ferroelectrics, volume 105 of Topics in Applied Physics, page 117. Springer-Verlag, Berlin, 2007.

${ }^{35}$ N. A. Spaldin. Analogies and differences between ferroelectrics and ferromagnets. In K. Rabe, C. H. Ahn, and J.-M. Triscone, editors, Physics of ferroelectrics, volume 105 of Topics in Applied Physics, page 175. Springer-Verlag, Berlin, 2007.

${ }^{36}$ T. Kimura. Annu. Rev. Mater. Res., 37:387, 2007.

${ }^{37}$ S.-W. Cheong and M. Mostovoy. Nature Mater., 6:13, 2007.

${ }^{38}$ C. N. R. Rao and C. R. Serrao. J. Mater. Chem., 17:4931, 2007.

${ }^{39}$ J. van den Brink and D. I. Khomskii. J. Phys.: Condens. Matter, 20:434217, 2008.

${ }^{40}$ D. I. Khomskii. Physics, 2:20, 2009.

${ }^{41}$ C. M. Teodorescu. Phys. Chem. Chem. Phys., 23:4085, 2021.

${ }^{42}$ R. D. King-Smith and D. Vanderbilt. Phys. Rev. B, 47:1651, 1993.

${ }^{43}$ D. Vanderbilt and R. D. King-Smith. Phys. Rev. B, 48:4442, 1993.

${ }^{44}$ R. Resta. Rev. Mod. Phys., 66:899, 1994.

${ }^{45}$ R. Resta. Ferroelectrics, 151:49, 1994.

${ }^{46} \mathrm{R}$. Resta and D. Vanderbilt. Theory of polarisation: a modern approach. In K. Rabe, C. H. Ahn, and J.-M. Triscone, editors, Physics of ferroelectrics, volume 105 of Topics in Applied Physics, page 31. Springer-Verlag, Berlin, 2007.

${ }^{47}$ N. A. Spaldin. Journal of Solid State Chemistry, 195:2, 2012.

${ }^{48}$ M. V. Berry. Proc. R. Soc. Lond. A, 392:45, 1984.

${ }^{49}$ D. J. Kim, J.Y. Jo, Y. S. Kim, Y. J. Chang, J. S. Lee, J.-G. Yoon T. K. Song, and T.W. Noh. Phys. Rev. Lett., 95:237602, 2005.

${ }^{50}$ M. Stengel and N. A. Spaldin. Nature, 443:679, 2006.

${ }^{51}$ L.-W. Chang, M. Alexe, J. F. Scott, and J. M. Gregg. Adv. Mater., 21:4911, 2009.

${ }^{52}$ M. Stengel, D. Vanderbilt, and N. A. Spaldin. Nature Materials, 8:392, 2009.

${ }^{53}$ Y. Wang, M. K. Niranjan, K. Janicka, J. P. Velev, M. Ye. Zhuravlev, S. S. Jaswal, and E. Y. Tsymbal1. Phys. Rev. B, 82:094114, 2010.

${ }^{54}$ P. Sun, Y.-Z. Wu, T.-Y. Cai, and S. Ju. Appl. Phys. Lett., 99:052901, 2011.

${ }^{55}$ Q. Yang, J. Cao, Y. Zhou, L. Sun, and X. Lou. Acta Materialia, 112:216, 2016.

${ }^{56}$ C. M. Teodorescu, L. Pintilie, N. G. Apostol, R. M. Costescu, G. A. Lungu, L. Hrib, L. Trupină, L. C. Tănase, I. C. Bucur, and A. E. Bocîrnea. Phys. Rev. B, 96:115438, 
Epitaxial ferroelectric interfacial devices

2017.

${ }^{57}$ G. A. Boni, C. F. Chirila, L. Hrib, R. Negrea, L. D. Filip, I. Pintilie, and L. Pintilie. Scientific Reports, 9:14698, 2019.

${ }^{58}$ W. L. Brown, 1957. U.S. Patent 2,791,759.

${ }^{59}$ D. H. Looney, 1957. U.S. Patent 2,791,758.

${ }^{60}$ J. F. Scott. Science, 315:954, 2007.

${ }^{61}$ J. R. Contreras, H. Kohlstedt, U. Poppe, R. Waser, C. Buchal, and N. A. Pertsev. Appl. Phys. Lett., 83:4595, 2003.

${ }^{62}$ M. Ye. Zhuravlev, S. S. Jaswal, E. Y. Tsymbal, and R. F. Sabirianov. Appl. Phys. Lett., $87: 222114,2005$.

${ }^{63}$ H. Kohlstedt, N. A. Pertsev, J. R Contreras, and R. Waser. Phys. Rev. B, 72:125341, 2005.

${ }^{64}$ J. P. Velev, C.-G. Duan, K. D. Belashchenko, S. S. Jaswal, and E.Y. Tsymbal. Phys. Rev. Lett., 98:137201, 2007.

${ }^{65}$ P. Maksymovych, S. Jesse, P. Yu, R. Ramesh, A. P. Baddorf, and S. V. Kalinin. Science, $324: 1421,2009$

${ }^{66}$ M. Ye. Zhuravlev, Y. Wang, S. Maekawa, and E. Y. Tsymbal. Appl. Phys. Lett., 95:052902, 2009.

${ }^{67}$ V. Garcia, S. Fusil, K. Bouzehouane, S. Enouz-Vedrenne, N. D. Mathur, A. Barthélémy, and M. Bibes. Nature, 460:81, 2009.

${ }^{68}$ A. Gruverman, D. Wu, H. Lu, Y. Wang, H. W. Jang, C. M. Folkman, M. Ye. Zhuravlev, D. Felker, M. Rzchowski, C.-B. Eom, and E. Y. Tsymbal. Nano Letters, 9:3539, 2009.

${ }^{69}$ R. Meservey and P. M. Tedrow. Phys. Rep., 238:173, 1994.

${ }^{70}$ W. J. Gallagher and S. S. P. Parkin. IBM J. Res. Dev., 50:5, 2006

${ }^{71}$ M. Lorenz, M. S. R. Rao, T. Venkatesan, E. Fortunato, P. Barquinha, R. Branquinho, D. Salgueiro, R. Martins, E. Carlos, A. Liu, F. K. Shan, M. Grundmann, H. Boschker, J. Mukherjee, M. Priyadarshini, N. DasGupta, D. J. Rogers, F. H. Teherani, E. V. Sandana, P. Bove, K. Rietwyk, A. Zaban, A. Veziridis, A. Weidenkaff, M. Muralidhar, M. Murakami, S. Abel, J. Fompeyrine, J. Zuniga-Perez, R. Ramesh, N. A. Spaldin, S. Ostanin, V. Borisov, I. Mertig, V. Lazenka, G. Srinivasan, W. Prellier, M. Uchida, M. Kawasaki, R. Pentcheva, P. Gegenwart, F. M. Granozio, J. Fontcuberta, and N. Pryds. J. Phys. D: Appl. Phys., 49:433001, 2016. 
Epitaxial ferroelectric interfacial devices

${ }^{72}$ A. Kalaboukhov and H. Boschker. Nanoscale patterning of complex-oxide materials. ${ }^{167}$, page 17.

${ }^{73}$ G. Cheng and J. Levy. Possible future quantum technologies based on correlated nanoelectronics. ${ }^{167}$, page 44.

${ }^{74} \mathrm{~J}$. Grollier. Oxide nano-electronics for neuromorphic computing. ${ }^{167}$, page 42.

${ }^{75}$ R. Dittmann. Resistive switching oxides for data storage. ${ }^{167}$, page 30.

${ }^{76}$ S. Saïghi, C. G. Mayr, T. Serrano-Gotarredona, H. Schmidt, G. Lecerf, J. Tomas, J. Grollier, S. Boyn, A. F. Vincent, D. Querlioz, S. La Barbera, F. Alibart, D. Vuillaume, O. Bichler, C. Gamrat, and B. Linares-Barranco. Frontiers in Neuroscience, 9:51, 2015.

${ }^{77}$ S. Boyn, J. Grollier, G. Lecerf, B. Xu, N. Locatelli, S. Fusil, S. Girod, C. Carrétéro, K. Garcia, S. Xavier, J. Tomas, L. Bellaiche, M. Bibes, A. Barthélémy, S. Saïghi, and V. Garcia. Nat. Commun., 8:14736, 2017.

${ }^{78}$ L. Chen, T.-Y. Wang, Y.-W. Dai, M.-Y. Cha, H. Zhu, Q.-Q. Sun, S.-J. Ding, P. Zhou, L. Chua, and D.W. Zhang. Nanoscale, 10:15826, 2018.

${ }^{79}$ K. Galatsis, C. Ahn, I. Krivorotov, P. Kim, R. Lake, K. L. Wang, and J. P. Chang. IEEE Journal on Exploratory Solid-State Computational Devices and Circuits, 1:19, 2015.

${ }^{80}$ A. H. C. Neto, F. Guinea, N. M. R. Peres, K. S. Novoselov, and A. K. Geim. Rev. Mod. Phys., 81:109, 2009.

${ }^{81}$ V. Meunier, A. G. Souza Filho, E. B. Barros, and M. S. Dresselhaus. Rev. Mod. Phys., Vol., 88:025005, 2016.

${ }^{82}$ W. Zhao, R. M. Ribeiro, and G. Eda. Acc. Chem. Res., 48:91, 2015.

${ }^{83}$ Y. Li, C. Chen, W. Li, X. Mao, H. Liu, J. Xiang, A. Nie, Z. Liu, W. Zhu, and H. Zeng. Adv. Electron. Mater., 6:2000061, 2020.

${ }^{84}$ R. Samal, G. Sanyal, B. Chakraborty, and C. S. Rout. J. Mater. Chem. A, 9:2560, 2021.

${ }^{85}$ X. Hong. J. Phys.: Condens. Matter, 28:103003, 2016.

${ }^{86}$ Y. Sun, R. Wang, and K. Liu. Appl. Phys. Rev., 4:011301, 2017.

${ }^{87}$ C. Zhou and Y. Chai. Adv. Electron. Mater., 3:1600400, 2017.

${ }^{88}$ J. Wang and W. Hu. Chin. Phys. B, 26:037106, 2017.

${ }^{89}$ W. Zhu, T. Low, H. Wang, P. Ye, and X. Duan. 2D Mater., 6:032004, 2019.

${ }^{90}$ K. T. Kang, J. Park, D. Suh, and W. S. Choi. Adv. Mater., 31:1803732, 2019.

${ }^{91}$ H. Ryu, K. Xu, D. Li, X. Hong, and W. Zhu. Appl. Phys. Lett., 117:080503, 2020. 
Epitaxial ferroelectric interfacial devices

${ }^{92}$ J. Chu, Y. Wang, X. Wang, K. Hu, G. Rao, C. Gong, C. Wu, H. Hong, X. Wang, K. Liu, C. Gao, and J. Xiong. Adv. Mater., 33:2004469, 2021.

${ }^{93}$ A. Lipatov, A. Fursina, T. H. Vo, P. Sharma, A. Gruverman, and A. Sinitskii. Adv. Electron. Mater., 3:1700020, 2017.

${ }^{94}$ S. Yan, H. Huang, Z. Xie, G. Ye, X.-X. Li, T. Taniguchi, K. Watanabe, Z. Han, X. Chen, J. Wang, and J.-H. Chen. ACS Appl. Mater. Interfaces, 11:42358, 2019.

${ }^{95}$ P.-C. Shen, C. Lin, H. Wang, K. H. Teo, and J. Kong. Appl. Phys. Lett., 116:033501, 2020.

${ }^{96}$ E. V. Milov and B. A. Strukov. Physics of the Solid State, 43:513, 2001.

${ }^{97}$ N. Ikeda, H. Ohsumi, K. Ohwada, K. Ishii, T. Inami, K. Kakurai, Y. Murakami, K. Yoshii, S. Mori, Y. Horibe, and H. Kitô. Nature, 436:1136, 2005.

${ }^{98}$ R. Takahashi and M. Lippmaa. Jpn. J. Appl. Phys., 57:0902A1, 2018.

${ }^{99}$ J. Robertson and C. W. Chen. Appl. Phys. Lett., 74:1168, 1999.

${ }^{100}$ B. Nagaraj, S. Aggarwal, and R. Ramesh. J. Appl. Phys., 90:375, 2001.

${ }^{101}$ J. J. Lee and S. B. Desu. Ferroelectric Letters, 20:27, 1995.

${ }^{102}$ S. K. Dey, J.-J. Lee, and P. Alluri. Jpn. J. Appl. Phys., 34:3142, 1995.

${ }^{103}$ L. Pintilie, I. Vrejoiu, D. Hesse, and M. Alexe. J. Appl. Phys., 104:114101, 2008.

${ }^{104}$ I. Pintilie, C. M. Teodorescu, C. Ghica, C. Chirila, A. G. Boni, L. Hrib, I. Pasuk, R. Negrea, N. Apostol, and L. Pintilie. ACS Appl. Mater. Interfaces, 6:2929, 2014.

${ }^{105}$ U. Rabe, M. Kopycinska, S. Hirsekorn, J. M. Saldaña, G. A. Schneider, and W. Arnold. J. Phys. D: Appl. Phys., 35:2621, 2002.

${ }^{106}$ A. Gruverman and A. Kholkin. Rep. Prog. Phys., 69:2443, 2006.

${ }^{107}$ S. V. Kalinin, A. N. Morozovska, L. Q. Chen, and B. J. Rodriguez. Rep. Prog. Phys., 73:056502, 2010 .

${ }^{108}$ E. Soergel. J. Phys. D: Appl. Phys., 44:464003, 2011.

${ }^{109}$ N. Balke, P. Maksymovych, S. Jesse, A. Herklotz, A. Tselev, C.-B. Eom, I. I. Kravchenko, and S. V. Kalinin. ACS Nano, 9:6484, 2015.

${ }^{110}$ A. G. Agronin, Y. Rosenwaks, and G. I. Rosenman. Nano Lett., 3:169, 2003.

${ }^{111}$ T. Jungk, Á. Hoffmann, and E. Soergel. Appl. Phys. Lett., 89:042901, 2006.

${ }^{112}$ T. Jungk, Á. Hoffmann, and E. Soergel. Appl. Phys. Lett., 91:253511, 2007.

${ }^{113}$ T. Jungk, Á. Hoffmann, and E. Soergel. Journal of Microscopy, 227:72, 2007.

${ }^{114}$ T. Jungk, Á. Hoffmann, and E. Soergel. Appl. Phys. A, 86:353, 2007. 
Epitaxial ferroelectric interfacial devices

${ }^{115}$ Á. Hoffmann, T. Jungk, and E. Soergel. Rev. Sci. Instrum., 78:016101, 2007.

${ }^{116}$ A. Labuda and R. Proksch. Appl. Phys. Lett., 106:253103, 2015.

${ }^{117}$ T. Tybell, P. Paruch, T. Giamarchi, and J.-M. Triscone. Phys. Rev. Lett., 89:097601, 2002.

${ }^{118}$ P. Paruch, T. Giamarchi, and J.-M. Triscone. Nanoscale studies of domain walls in epitaxial ferroelectric thin films. In K. Rabe, C. H. Ahn, and J.-M. Triscone, editors, Physics of ferroelectrics, volume 105 of Topics in Applied Physics, page 339. SpringerVerlag, Berlin, 2007.

${ }^{119}$ P. Sutter and E. Sutter. Adv. Funct. Mater., 23:2617, 2013.

${ }^{120}$ J. E. Rault, T. O. Menteş, A. Locatelli, and N. Barret. Scientific Reports, 4:6792, 2014.

${ }^{121}$ N. Barrett, J. E. Rault, J. L. Wang, C. Mathieu, A. Locatelli, T. O. Mentes, M. A. Niño, S. Fusil, M. Bibes, A. Barthélémy, D. Sando, W. Ren, S. Prosandeev, L. Bellaiche, B. Vilquin, A. Petraru, I. P. Krug, and C. M. Schneider. J. Appl. Phys., 113:187217, 2013.

${ }^{122}$ C. A. F. Vaz, A. Kleibert, and M. El Kazzi. Nanoscale xpeem spectromicroscopy. In K. D. Sattler, editor, 21st CENTURY NANOSCIENCE - A HANDBOOK, chapter 17. Taylor \& Francis (CRC Press), 2020.

${ }^{123}$ S. Cherifi, R. Hertel, S. Fusil, H. Béa, K. Bouzehouane, J. Allibe, M. Bibes, and A. Barthélémy. Phys. Status Solidi RRL, 4:22, 2010.

${ }^{124}$ R. Moubah, M. Elzo, S. El Moussaoui, D. Colson, N. Jaouen, R. Belkhou, and M. Viret. Appl. Phys. Lett., 100:042406, 2012.

${ }^{125}$ J. E. Rault, W. Ren, S. Prosandeev, S. Lisenkov, D. Sando, S. Fusil, M. Bibes, A. Barthélémy, L. Bellaiche, and N. Barrett. Phys. Rev. Lett., 109:267601, 2012.

${ }^{126}$ A. Sander, M. Christl, C.-T. Chiang, M. Alexe, and W. Widdra. J. Appl. Phys., 118:224102, 2015.

${ }^{127}$ D. D. Fong, G. B. Stephenson, S. K. Streiffer, J. A. Eastman, O. Auciello, P. H. Fuoss, and C. Thompson. Science, 304:1650, 2004.

${ }^{128}$ D. D. Fong and C. Thompson. Annu. Rev. Matter. Res., 36:431, 2006.

${ }^{129}$ G. Catalan, A. H. G. Vlooswijk, A. Janssens, G. Rispens, S. Redfern, G. Rijnders, D. H. A. Blank, and B. Noheda. Integrated Ferroelectrics, 92:18, 2007.

${ }^{130}$ E. A. Kraut, R. W. Grant, J. R. Waldrop, and S. P. Eowalczyk. Phys. Rev. Lett., 44:1620, 1980. 
Epitaxial ferroelectric interfacial devices

${ }^{131}$ A. D. Katnani and G. Margaritondo. Phys. Rev. B, 28:1944, 1983.

${ }^{132}$ J. R. Waldrop, R. W. Grant, S. P. Kowalczyk, and E. A. Kraut. J. Vac. Sci. Technol. $A, 3: 835,1985$.

${ }^{133}$ J. Zegenhagen. Eur. Phys. J. Appl. Phys., 70:20701, 2015.

${ }^{134}$ M.-A. Husanu and C. A. F. Vaz. Spectroscopic characterisation of multiferroic interfaces. In C. Cancellieri and V. N. Strocov, editors, Spectroscopy of complex oxide interfaces, volume 266 of Springer Series in Materials Science, page 245. Springer, Cham, 2018.

${ }^{135}$ R. V. Vedrinskii, V. L. Kraizman, A. A. Novakovich, Ph. V. Demekhin, S. V. Urazhdin, B. Ravel, and E. A. Stern. J. Phys. IV France, 7:C2-107, 1997.

${ }^{136}$ B. Ravel, E. A. Stern, R. I. Vedrinskii, and V. Kraizman. Ferroelectrics, 206:407, 1998.

${ }^{137}$ F. M. F. de Groot. Chem. Rev., 101:1779, 2001.

${ }^{138}$ D. Cao, I.-K. Jeong, R. H. Heffner, T. Darling, J.-K. Lee, F. Bridges, J.-S. Park, and K.-S. Hong. Phys. Rev. B, 70:224102, 2004.

${ }^{139}$ A. Mesquita, A. Michalowicz, and V. R. Mastelaro. Journal of Physics: Conference Series, 190:012081, 2009.

${ }^{140}$ A. Al-Zein, G. Fraysse, J. Rouquette, Ph. Papet, J. Haines, B. Hehlen, C. Levelut, G. Aquilanti, and Y. Joly. Phys. Rev. B, 81:174110, 2010.

${ }^{141}$ E. Arenholz, G. van der Laan, A. Fraile-Rodríguez, P. Yu, Q. He, and R. Ramesh. Phys. Rev. B, 82:140103(R), 2010.

${ }^{142}$ H. N. Chapman, A. Barty, M. J. Bogan, S. Boutet, M. Frank, S. P. Hau-Riege, S. Marchesini, B. W. Woods, S. Bajt, W. H. Benner, R. A. London, E. Plönjes, M. Kuhlmann, R. Treusch, S. Düsterer, T. Tschentscher, J. R. Schneider, E. Spiller, T. Möller, C. Bostedt, M. Hoener, D. A. Shapiro, K. O. Hodgson, D. van der Spoel, F. Burmeister, M. Bergh, C. Caleman, G. Huldt, M. M. Seibert, F. R. N. C. Maia, R. W. Lee, A. Szöke, N. Timneanu, and J. Hajdu. Nature Physics, 2:839, 2006.

${ }^{143}$ T. F. Nova, A. S. Disa, M. Fechner, and A. Cavalleri. Science, 364:1075, 2019.

${ }^{144}$ S. R. Spurgeon. Scanning transmission electron microscopy of oxide interfaces and heterostructures. arXiv, 2020.

${ }^{145}$ D. Su, Q. Meng, C. A. F. Vaz, M.-G. Han, Y. Segal, F. J. Walker, M. Sawicki, C. Broadbridge, and C. H. Ahn. Appl. Phys. Lett., 99:102902, 2011.

${ }^{146}$ P. Gao, C. T. Nelson, J. R. Jokisaari, Y. Zhang, S.-H. Baek, C. W. Bark, E. Wang, Y. Liu, C.-B. Eom, and X. Pan. Adv. Mater., 24:1106, 2012. 
Epitaxial ferroelectric interfacial devices

${ }^{147}$ S. R. Spurgeon, P. V. Balachandran, D. M. Kepaptsoglou, A. R. Damodaran, J. Karthik, S. Nejati, L. Jones, H. Ambaye, V. Lauter, Q. M. Ramasse, K. K. S. Lau, L. W. Martin, J. M. Rondinelli, and M. L. Taheri. Nature Communications, 6:6735, 2015.

${ }^{148}$ W. Zhong, R. D. King-Smith, and D. Vanderbilt. Phys. Rev. Lett., 72:3618, 1994.

${ }^{149}$ P. Chen, Z. Huang, C. Li, B. Zhang, N. Bao, P. Yang, X. Yu, S. Zeng, C. Tang, X. Wu, J. Chen, J. Ding, S. J. Pennycook, A. Ariando, T. V. Venkatesan, and G. M. Chow. Adv. Funct. Mater., 28:1801766, 2018.

${ }^{150}$ C. T. Nelson, P. Gao, J. R. Jokisaari, C. Heikes, C. Adamo, A. Melville, S.-H. Baek, C. M. Folkman, B. Winchester, Y. Guv Y. Liu, K. Zhang, E. Wang, J. Li, L.-Q. Chen, C.-B. Eom, D. G. Schlom, and X. Pan. Science, 334:968, 2011.

${ }^{151}$ P. Gao, C. T. Nelson, J. R. Jokisaari, S.-H. Baek, C. W. Bark, Y. Zhang, E. Wang, D. G. Schlom, C.-B. Eom, and X. Pan. Nature Communications, 2:591, 2011.

${ }^{152}$ P. Gao, J. Britson, C. T. Nelson, J. R. Jokisaari, C. Duan, M. Trassin, S.-H. Baek, H. Guo, L. Li, Y. Wang, Y.-H. Chu, A. M. Minor, C.-B. Eom, R. Ramesh, L.-Q. Chen, and X. Pan. Nature Communications, 5:3801, 2014.

${ }^{153}$ H. J. Chang, S. V. Kalinin, A. N. Morozovska, M. Huijben, Y.-H. Chu, P. Yu, R. Ramesh, E. A. Eliseev, G. S. Svechnikov, S. J. Pennycook, and A. Y. Borisevich. Adv. Mater., 23:2474, 2011.

${ }^{154}$ Y.-M. Kim, A. Kumar, A. Hatt, A. N. Morozovska, A. Tselev, M. D. Biegalski, I. Ivanov, E. A. Eliseev, S. J. Pennycook, J. M. Rondinelli, S. V. Kalinin, and A. Y. Borisevich. Adv. Mater., 25:2497, 2013.

${ }^{155}$ D. Zheng, J. Zhang, Y. Wang, Z. Wang, W. Zheng, D. Li, L. Zhang, C. Jin, P. Li, X. Zhang, and H. Bai. ACS Appl. Mater. Interfaces, 10:40328, 2018.

${ }^{156}$ S. R. Spurgeon, J. D. Sloppy, D. M. Kepaptsoglou, P. V. Balachandran, S. Nejati, J. Karthik, A. R. Damodaran, C. L. Johnson, H. Ambaye, R. Goyette, V. Lauter, Q. M. Ramasse, J. C. Idrobo, K. K. S. Lau, S. E. Lofland, Jr., J. M. Rondinelli, L. W. Martin, and M. L. Taheri. ACS Nano, 8:894, 2014.

${ }^{157}$ R. Soni, A. Petraru, H. S. Nair, O. Vavra, M. Ziegler, S. K. Kim, D. S. Jeong, and H. Kohlstedt. Nanoscale, 8:10799, 2016.

${ }^{158}$ Q. Meng, G. Xu, H. Xin, E. A. Stach, Y. Zhu, and D. Su. J. Phys. Chem. A, 123:4632, 2019. 
Epitaxial ferroelectric interfacial devices

${ }^{159}$ H. Guo, Z. Wang, S. Dong, S. Ghosh, M. Saghayezhian, L. Chen, Y. Weng, A. Herklotz, T. Z. Ward, R. Jin, S. T. Pantelides, Y. Zhub, J. Zhang, and E. W. Plummer. PNAS, 114:E5062, 2017.

${ }^{160}$ H. Wang, X. Chi, Z. Liu, H Yoong, L Tao, J Xiao, R Guo, J Wang, Z Dong, P Yang, C.-J. Sun, C. Li, X. Yan, J. Wang, G. M. Chow, E. Y. Tsymbal, H. Tian, and J. Chen. Nano Lett., 19:3057, 2019.

${ }^{161}$ M. Marinova, J. E. Rault, A. Gloter, S. Nemsak, G. K. Palsson, J.-P. Rueff, C. S. Fadley, C. Carrétéro, H. Yamada, K. March, V. Garcia, S. Fusil, A. Barthélémy, O. Stéphan, C. Colliex, and M. Bibes. Nano Lett., 15:2533, 2015.

${ }^{162}$ Z. Yuan, J. Ruan, L. Xie, X. Pan, D. Wu, and P. Wang. Appl. Phys. Lett., 110:171602, 2017.

${ }^{163}$ D. G. Schlom, J.H. Haeni, J. Lettieri, C. D. Theis, W. Tian, J. C. Jiang, and X. Q. Pan. Mater. Sci. Eng. B, 87:282, 2001.

${ }^{164}$ A.-B. Posadas, M. Lippmaa, F. J. Walker, M. Dawber, C. H. Ahn, and J.-M. Triscone. Growth and novel applications of epitaxial oxide thin films. In K. Rabe, C. H. Ahn, and J.-M. Triscone, editors, Physics of ferroelectrics, volume 105 of Topics in Applied Physics, page 219. Springer-Verlag, Berlin, 2007.

${ }^{165}$ N. Izyumskaya, Y.-I. Alivov, S.-J. Cho, H. Morkoç, and H. Lee Y.-S. Kang. Critical Reviews in Solid State and Materials Sciences, 32:111, 2007.

${ }^{166}$ N. Izyumskaya, Ya. Alivov, and H. Morkoç. Critical Reviews in Solid State and Materials Sciences, 34:89, 2009.

${ }^{167}$ M. Coll, J. Fontcuberta, M. Althammer, M. Bibes, H. Boschker, A. Calleja, G. Cheng, M. Cuoco, R. Dittmann, B. Dkhil, I. El Baggari, M. Fanciulli, I. Fina, E. Fortunato, C. Frontera, S. Fujita, V. Garcia, S. T. B. Goennenwein, C.-G. Granqvist, J. Grollier, R. Gross, A. Hagfeldt, G. Herranz, K. Hono, E. Houwman, M. Huijben, A. Kalaboukhov, D. J. Keeble, G. Koster, L. F. Kourkoutis, J. Levy, M. Lira-Cantu, J. L. MacManus-Driscoll, J. Mannhart, R. Martins, S. Menzel, T. Mikolajick, M. Napari, M. D. Nguyen, G. Niklasson, C. Paillard, S. Panigrahi, G. Rijnders, F. Sánchez, P. Sanchis, S. Sanna, D. G. Schlom, U. Schroeder, K. M. Shen, A. Siemon, M. Spreitzer, H. Sukegawa, R. Tamayo, J. van den Brink, N. Pryds, and F. M. Granozio. Applied Surface Science, 482:1, 2019.

${ }^{168}$ L. Shi and J. Ni. Phys. Rev. Lett., 97:126105, 2006. 
Epitaxial ferroelectric interfacial devices

${ }^{169}$ M. Adachi, Y. Akishige, K. Deguchi, T. Ikeda, M. Okuyama, A. Sakai, E. Sawaguchi, T. Takenaka, T. Tsukamoto, and T. Yagi. Perovskite-type oxides. In Y. Shiozaki, E. Nakamura, and T. Mitsui, editors, Ferroelectrics and Related Substances, volume 36A1 of Landolt-Börnstein - Group III: Crystal and Solid State Physics, page 67. SpringerVerlag, Berlin, 2001.

${ }^{170}$ J. Robertson. Eur. Phys. J. Appl. Phys., 28:265, 2004.

${ }^{171}$ S. Li, F. Chen, R. Schafranek, T. J. M. Bayer, K. Rachut, A. Fuchs, S. Siol, M. Weidner, M. Hohmann, V. Pfeifer, J. Morasch, C. Ghinea, E. Arveux, R. Günzler, J. Gassmann, C. Körber, Y. Gassenbauer, F. Säuberlich, G. V. Rao, S. Payan, M. Maglione, C. Chirila, L. Pintilie, L. Jia, K. Ellmer, M. Naderer, K. Reichmann, U. Böttger, S. Schmelzer, R. C. Frunza, H. Uršič, B. Malič, W.-B. Wu, P. Erhart, and A. Klein. Phys. Status Solidi RRL, $8: 571,2014$.

${ }^{172}$ H. Hidaka. Embedded magnetic RAM. In K. Zhang, editor, Embedded Memories for Nano-Scale VLSIs, Series on Integrated Circuits and Systems, page 241. Springer-Verlag, 2009.

${ }^{173}$ M. H. Park, Y. H. Lee, H. J. Kim, Y. J. Kim, T. Moon, K. D. Kim, J. Müller, A. Kersch, U. Schroeder, T. Mikolajick, and C. S. Hwang. Adv. Mater., 27:1811, 2015.

${ }^{174}$ M. J. Haun, E. Furman, H. A. McKinstry, and L. E. Cross. Ferroelectrics, 99:27, 1989. ${ }^{175}$ S. E. Reyes-Lillo, K. F. Garrity, and K. M. Rabe. Phys. Rev. B, 90:140103(R), 2014. ${ }^{176}$ D. Lebeugle, D. Colson, A. Forget, and M. Viret. Appl. Phys. Lett., 91:022907, 2007. ${ }^{177}$ J. F. Scott, K. Watanabe, A. J. Hartmann, and R. N. Lamb. Ferroelectrics, 225:83, 1999. ${ }^{178}$ F. Charra and G. G. Gurzadyan. In D. F. Nelson, editor, High Frequency Properties of Dielectric Crystals, volume 30B of Landolt-Börnstein - Group III: Condensed Matter. Springer-Verlag, Berlin, 2000.

${ }^{179}$ A. Kumar, R. C. Rai, N. J. Podraza, S. Denev, M. Ramirez, Y.-H. Chu, L. W. Martin, J. Ihlefeld, T. Heeg, J. Schubert, D. G. Schlom, J. Orenstein, R. Ramesh, R. W. Collins, J. L. Musfeldt, and V. Gopalan. Appl. Phys. Lett., 92:121915, 2008.

${ }^{180}$ C. H. Peng, J.-F. Chang, and S. B. Desu. Mat. Res. Soc. Symp. Proc., 243:21, 1992.

${ }^{181}$ V. A. Shvets, V. N. Kruchinin, and V. A. Gritsenko. Optics and Spectroscopy, 123:728, 2017.

${ }^{182}$ T. V. Perevalov, A. K. Gutakovskii, V. N. Kruchinin, V. A. Gritsenko, and I. P. Prosvirin. Mater. Res. Express, 6:036403, 2019. 
Epitaxial ferroelectric interfacial devices

${ }^{183}$ J. Fujisawa, T. Eda, and M. Hanaya. Chemical Physical Letters, 685:23, 2017.

${ }^{184}$ R. Schafranek, S. Li, F. Chen, W. Wu, and A. Klein. Phys. Rev. B, 84:045317, 2011.

${ }^{185}$ M. J. Haun, E. Furman, S. J. Jang, and L. E. Cross. Ferroelectrics, 99:63, 1989.

${ }^{186}$ I. Vrejoiu, G. Le Rhun, L. Pintilie, D. Hesse, M. Alexe, and U. Gösele. Adv. Mater., 18:1657, 2006.

${ }^{187}$ J. Chakrabartty, R. Nechache, C. Harnagea, S. Li, and F. Rosei. Nanotechnology, $27: 215402,2016$.

${ }^{188}$ Y. Peng, G. Han, W. Xiao, Y. Liu, Q. Li, C. Zhang, J. Zhang, and Y. Hao. Superlattices and Microstructures, 130:519, 2019.

${ }^{189}$ S. M. Sze. Physics of Semiconductor Devices. John Wiley \& Sons, New York, 2nd edition, 1981.

${ }^{190}$ B. W. Wessels. Annu. Rev. Mater. Res., 37:659, 2007.

${ }^{191}$ A. Rosa, D. Tulli, P. Castera, A. M. Gutierrez, A. Griol, M. Baquero, B. Vilquin, F. Eltes, S. Abel, J. Fompeyrine, and P. Sanchis. Optical Materials Express, 7:4328, 2017.

${ }^{192}$ M. Acosta, N. Novak, V. Rojas, S. Patel, R. Vaish, J. Koruza, Jr. G. A. Rossetti, and J. Rödel. Appl. Phys. Rev., 4:041305, 2017.

${ }^{193}$ V. Buscaglia, M. T. Buscaglia, and G. Canu. BaTiO ${ }_{3}$-based ceramics: fundamentals, properties and applications. In Encyclopedia of Materials: Technical ceramics and glasses, page 1. Elsevier, 2020.

${ }^{194}$ L. A. Shebanov. phys. stat. sol. (a), 65:321, 1981.

${ }^{195}$ T. Terashima, Y. Bando, K. Ijima, K. Yamamoto, K. Hirata, K. Hayashi, K. Kamigaki, and H. Terauchi. Phys. Rev, Lett., 65:2684, 1990.

${ }^{196}$ W. J. Lin, T. Y. Tseng, H. B. Lu, S. L. Tu, S. J. Yang, and I. N. Lin. J. Appl. Phys., 77:6466, 1995.

${ }^{197}$ Y. Ishibashi, T. Tsurumi, N. Ohashi, and O. Fukunaga. Solid State Ionics, 108:91, 1998. ${ }^{198}$ W. Tian, J. H. Haeni, D. G. Schlom, and X. Q. Pan. Mat. Res. Soc. Symp. Proc., 655:CC7.8.1, 2001.

${ }^{199}$ Y. S. Kim, D. H. Kim, J. D. Kim, Y. J. Chang, T. W. Noh, J. H. Kong, K. Char, Y. D. Park, S. D. Bu, J.-G. Yoon, and J.-S. Chung. Appl. Phys. Lett., 86:102907, 2005.

${ }^{200}$ X. Chen, S. Yang, J.-H. Kim, H.-D. Kim, J.-S. Kim, G. Rojas, R. Skomski, H. Lu, A. Bhattacharya, T. Santos, N. Guisinger, M. Bode, A. Gruverman, and A. Enders. New Journal of Physics, 13:083037, 2011. 
Epitaxial ferroelectric interfacial devices

${ }^{201}$ G. Niu, B. Gautier, S. Yin, G. Saint-Girons, P. Lecoeur, V. Pillard, G. Hollinger, and B. Vilquin. Thin Solid Films, 520:4595, 2012.

${ }^{202}$ H. S. Wang, K. Ma, D. F. Cui, Z. Q. Peng, Y. L. Zhou, H. B. Lu, Z. H. Chen, L. Li, and G. Z. Yang. J. Crystal Growth, 177:67, 1997.

${ }^{203}$ A. Zhu, C. Li, K. Zhou, Y. Zheng, H. Lu, H. Wang, D. Cui, Z. Chen, and Z. Mai. J. Materials Science Letters, 16:1898, 1997.

${ }^{204}$ G. H. Lee, M. Yoshimoto, T. Ohnishi, K. Sasaki, and H. Koinuma. Materials Science and Engineering B, 56:213, 1998.

${ }^{205}$ T. Suzuki, Y. Nishi, and M. Fujimoto. Philos. Mag. A, 79:2461, 1999.

${ }^{206}$ C. L. Li, Z. H. Chen, Y. L. Zhou, and D. F. Cui. J. Phys.: Condens. Matter, 13:5261, 2001.

${ }^{207}$ G. H Lee, B. C. Shin, and I. S. Kim. Materials Letters, 50:134, 2001.

${ }^{208}$ A. Visinoiu, M. Alexe, H. N. Lee, D. N. Zakharov, A. Pignolet, D. Hesse, and U. Gösele. Mat. Res. Soc. Symp. Proc., 688:C8.4.1, 2002.

${ }^{209}$ J. Y. Jo, Y. S. Kim, D. H. Kim, J. D. Kim, Y. J. Chang, J. H. Kong, Y. D. Park, T. K. Song, J.-G. Yoon, J. S. Jung, and T. W. Noh. Thin Solid Films, 486:149, 2005.

${ }^{210}$ A. Petraru, N. A. Pertsev, H. Kohlstedt, U. Poppe, R. Waser, A. Solbach, and U. Klemradt. J. Appl. Phys., 101:114106, 2007.

${ }^{211}$ P. S. S. R. Krishnan and P. R. Munroe. J. Asian Ceramic Societies, 1:149, 2013.

${ }^{212}$ R. Takahashi and M. Lippmaa. ACS Appl. Mater. Interfaces, 9:21314, 2017.

${ }^{213}$ F. Ambriz-Vargas, I. Velasco-Davalos, R. Thomas, and A. Ruediger. J. Vac. Soc. Technol. B, 34:02M101, 2016.

${ }^{214}$ Y. J. Shin, J. Jiang, Y. Jia, F. J. Walker, and C. H. Ahn. APL Materials, 9:041104, 2021.

${ }^{215}$ Y. Yano, K. Iijima, Y. Daitoh, T. Terashima, Y. Bando, Y. Watanabe, H. Kasatani, and H. Terauchi. J. Appl. Phys., 76:7833, 1994.

${ }^{216}$ J. H. Kim and S. Hishita. Journal of Materials Science, 30:4645, 1995.

${ }^{217}$ Yu. A. Boikov and T. Claeson. J. Appl. Phys., 89:5053, 2001.

${ }^{218}$ K. J. Choi, M. Biegalski, Y. L. Li, A. Sharan, J. Schubert, R. Uecker, P. Reiche, Y. B. Chen, X. Q. Pan, V. Gopalan, L.-Q. Chen, D. G. Schlom, and C. B. Eom. Science, $306: 1005,2004$ 
Epitaxial ferroelectric interfacial devices

${ }^{219}$ W. Zhang, L. Kang, M. Yuan, Q. Yang, and J. Ouyang. Journal of Alloys and Compounds, 580:363, 2013.

${ }^{220}$ J. Schubert, O. Trithaveesak, A. Petraru, C. L. Jia, R. Uecker, P. Reiche, and D. G. Schlom. Appl. Phys. Lett., 82:3460, 2003.

${ }^{221}$ C. H. Lei. Thin Solid Films, 515:1701, 2006.

${ }^{222}$ D. M. Hwang, R. Ramesh, C. Y. Chen, X. D. Wu, A. Inam, M. S. Hegde, B. Wilkens, C. C. Chang, L. Nazar, T. Venkatesan, S. Miura, S. Matsubara, Y. Miyasaka, and N. Shohata. J. Appl. Phys., 68:1772, 1990.

${ }^{223}$ L. H. Chang and W. A. Anderson. Appl. Surf. Sci., 92:52, 1996.

${ }^{224}$ J. Hallmark, Z. Yu, R. Droopad, J. Ramdani, J. Curless, C. Overgaard, J. Finder, D. Marshall, J. Wang, and B. Ooms. Integrated Ferroelectrics, 27:41, 1999.

${ }^{225}$ V. Vaithyanathan, J. Lettieri, W. Tian, A. Sharan, A. Vasudevarao, Y. L. Li, A. Kochhar, H. Ma, J. Levy, P. Zschack, J. C. Woicik, L. Q. Chen, V. Gopalan, and D. G. Schlom. J. Appl. Phys., 100:024108, 2006.

${ }^{226}$ L. Qiao and X. Bi. Appl. Phys. Lett., 92:062912, 2008.

${ }^{227}$ G. Niu, S. Yin, G. Saint-Girons, B. Gautier, P. Lecoeur, V. Pillard, G. Hollinger, and B. Vilquin. Microelectronic Engineering, 88:1232, 2011.

${ }^{228}$ C. Dubourdieu, J. Bruley, T. M. Arruda, A. Posadas, J. Jordan-Sweet, M. M. Frank, E. Cartier, D. J. Frank, S. V. Kalinin, A. A. Demkov, and V. Narayanan. Nature Nanotechnology, 8:748, 2013.

${ }^{229}$ S. R. Singamaneni, W. Fan, J. T. Prater, and J. Narayan. J. Appl. Phys., 116:224104, 2014 .

${ }^{230}$ J. H. Ngai, D. P. Kumah, C. H. Ahn, and F. J. Walker. Appl. Phys. Lett., 104:062905, 2014 .

${ }^{231}$ L. Kornblum, M. D. Morales-Acosta, E. N. Jin, C. H. Ahn, and F. J. Walker. Adv. Mater. Interfaces, 2:1500193, 2015.

${ }^{232}$ E. L. Lin, A. B. Posadas, H. W. Wu, D. J. Smith, A. A. Demkov, and J. G. Ekerdt. J. Crystal Growth, 476:6, 2017.

${ }^{233}$ Z. Wang, T. Yasuda, S. Hatatani, and S. Oda. Jpn. J. Appl. Phys., 38:6817, 1999.

${ }^{234}$ S. Tinte, K. M. Rabe, and D. Vanderbilt. Phys. Rev. B, 68:144105, 2003.

${ }^{235}$ H. Morioka, S. Yokoyama, T. Oikawa, H. Funakubo, and K. Saito. Appl. Phys. Lett., 85:3516, 2004. 
Epitaxial ferroelectric interfacial devices

${ }^{236}$ C. Ederer and N. A. Spaldin. Phys. Rev. Lett., 95:257601, 2005.

${ }^{237}$ A. A. Belik, S. Iikubo, K. Kodama, N. Igawa, S. Shamoto, S. Niitaka, M. Azuma, Y. Shimakawa, M. Takano, F. Izumi, and E. Takayama-Muromachi. Chem. Mater., 18:798, 2006.

${ }^{238}$ R. J. Zeches, M. D. Rossell, J. X. Zhang, A. J. Hatt, Q. He, C.-H. Yang, A. Kumar, C. H. Wang, A. Melville, C. Adamo, G. Sheng, Y.-H. Chu, J. F. Ihlefeld, R. Erni, C. Ederer, V. Gopalan, L. Q. Chen, D. G. Schlom, N. A. Spaldin, L. W. Martin, and R. Ramesh. Science, 326:977, 2009.

${ }^{239}$ H. Béa, B. Dupé, S. Fusil, R. Mattana, E. Jacquet, B. Warot-Fonrose, F. Wilhelm, A. Rogalev, S. Petit, V. Cros, A. Anane, F. Petroff, K. Bouzehouane, G. Geneste, B. Dkhil, S. Lisenkov, I. Ponomareva, L. Bellaiche, M. Bibes, and A. Barthélémy. Phys. Rev. Lett., 102:217603, 2009.

${ }^{240}$ L. Zhang, J. Chen, L. Fan, O. Diéguez, J. Cao, Z. Pan, Y. Wang, J. Wang, M. Kim, S. Deng, J. Wang, H. Wang, J. Deng, R. Yu, J. F. Scott, and X. Xing. Science, 361:494, 2018.

${ }^{241}$ A. Visinoiu, M. Alexe, H. N. Lee, D. N. Zakharov, A. Pignolet, D. Hesse, and U. Gösele. J. Appl. Phys., 91:10157, 2002.

${ }^{242}$ J. Shin, S. V. Kalinin, A. Y. Borisevich, E. W. Plummer, and A. P. Baddorf. Appl. Phys. Lett., 91:202901, 2007.

${ }^{243}$ M. Kawai, D. Kan, S. Isojima, H. Kurata, S. Isoda, Y. Shimakawa, S. Kimura, and O. Sakata. J. Appl. Phys., 102:114311, 2007.

${ }^{244}$ T. Zhao, F. Chen, H. Lu, G. Yang, and Z. Chen. J. Appl. Phys., 87:7442, 2000.

${ }^{245}$ M. Arredondo, M. Saunders, A. Petraru, H. Kohlstedt, I. Vrejoiu, M. Alexe, D. Hesse, N. D. Browning, P. Munroe, and V. Nagarajan. J. Mater. Sci., 44:5297, 2009.

${ }^{246}$ L. Li. Materials Science and Engineering, 29:153, 2000.

${ }^{247}$ W. Pies and A. Weiss. Crystal structure data of inorganic compounds. volume 7b1 of Landolt-Börnstein - Group III: Crystal and Solid State Physics. Springer-Verlag, Berlin, 1975.

${ }^{248}$ R. Allmann, W. Pies, and A. Weiss. Crystal structure data of inorganic compounds. volume 7d2 of Landolt-Börnstein - Group III: Crystal and Solid State Physics. SpringerVerlag, Berlin, 1980. 
Epitaxial ferroelectric interfacial devices

${ }^{249}$ W. Pies and A. Weiss. Crystal structure data of inorganic compounds. volume 7 e of Landolt-Börnstein - Group III: Crystal and Solid State Physics. Springer-Verlag, Berlin, 1976.

${ }^{250}$ S. Geller. Acta Cryst., 10:243, 1957.

${ }^{251}$ J.W. Reiner, K. F. Garrity, F. J. Walker, S. Ismail-Beigi, and C. H. Ahn. Phys. Rev. Lett., 101:105503, 2008.

${ }^{252}$ J. W. Reiner, A. M. Kolpak, Y. Segal, K. F. Garrity, S. Ismail-Beigi, C. H. Ahn, and F. J. Walker. Adv. Mater., 22:2919, 2010.

${ }^{253}$ S.-H. Baek and C.-B. Eom. Acta Materialia, 61:2734, 2013.

${ }^{254}$ L. Mazet, S. M. Yang, S. V. Kalinin, S. Schamm-Chardon, and C. Dubourdieu. Sci. Technol. Adv. Mater., 16:036005, 2015.

${ }^{255}$ Y. Kim, C. Pham, and J. P. Chang. J. Phys. D: Appl. Phys., 48:063001, 2015.

${ }^{256}$ J. H. Ngai, K. Ahmadi-Majlan, J. Moghadam, M. Chrysler, D. Kumah, F. J. Walker, C.H. Ahn, T. Droubay, Y. Du, S.A. Chambers, M. Bowden, X. Shen, and D. Su. J. Mater. Res., 32:249, 2017.

${ }^{257}$ K. J. Kormondy, Y. Popoff, M. Sousa, F. Eltes, D. Caimi, M. D. Rossell, M. Fiebig, P. Hoffmann, C. Marchiori, M. Reinke, M. Trassin, A. A. Demkov, J. Fompeyrine, and S. Abel. Nanotechnology, 28:075706, 2017.

${ }^{258}$ S. R. Singameneni, J. T. Prater, and J. Narayan. Appl. Phys. Rev., 3:031301, 2016.

${ }^{259}$ D. P. Kumah, J. H. Ngai, and L. Kornblum. Adv. Funct. Mater., 30:1901597, 2020.

${ }^{260}$ M. Spreitzer, D. Klement, T. P. Potǒcnik, U. Trstenjak, Z. Jovanović, M. D. Nguyen, H. Yuan, J. E. ten Elshof, E. Houwman, G. Koster, G. Rijnders, J. Fompeyrine, L. Kornblum, D. P. Fenning, Y. Liang, W.-Y. Tong, and P. Ghosez. APL Mater., 9:040701, 2021.

${ }^{261}$ M. B. Nardelli, F. J. Walker, and R. A. McKee. phys. stat. sol. (b), 241:2279, 2004.

${ }^{262}$ F. J. Walker and R. A. McKee. High-k crystalline gate dielectrics: a research perspective. In H. R. Huff and D. C. Gilmer, editors, High dielectric constant materials, page 607. Springer-Verlag, Berlin, 2005.

${ }^{263}$ J. Robertson. New high-k materials for cmos applications. In R. Fornari, editor, Comprehensive Semiconductor Science and Technology, volume 4, page 132. Elsevier B.V., 2011.

${ }^{264}$ R. A. McKee, F. J. Walker, and M. F. Chisholm. Phys. Rev. Lett., 81:3014, 1998. 
Epitaxial ferroelectric interfacial devices

${ }^{265}$ R. A. McKee, F. J. Walker, and M. F. Chisholm. Science, 293:468, 2001.

${ }^{266}$ C. Marchiori, M. Sousa, A. Guiller, H. Siegwart, J.-P. Locquet, J. Fompeyrine, G. J. Norga, and J. W. Seo. Appl. Phys. Lett., 88:072913, 2006.

${ }^{267}$ G. Delhaye, C. Merckling, M. El-Kazzi, G. Saint-Girons, M. Gendry, Y. Robach, G. Hollinger, L. Largeau, and G. Patriarche. J. Appl. Phys., 100:124109, 2006.

${ }^{268}$ Y. Segal, J.W. Reiner, A. M. Kolpak, Z. Zhang, S. Ismail-Beigi, C. H. Ahn, and F. J. Walker. Phys. Rev. Lett., 102:116101, 2009.

${ }^{269}$ P. Ponath, K. Fredrickson, A. B. Posadas, Y. Ren, X. Wu, R. K. Vasudevan, M. B. Okatan, S. Jesse, T. Aoki, M. R. McCartney, D. J. Smith, S. V. Kalinin, K. Lai, and A. A. Demkov. Nature Communications, 6:6067, 2015.

${ }^{270}$ D. P. Kumah, M. Dogan, J. H. Ngai, D. Qiu, Z. Zhang, D. Su, E. D. Specht, S. IsmailBeigi, C. H. Ahn, and F. J. Walker. Phys. Rev. Lett., 116:106101, 2016.

${ }^{271}$ A. S. Disa, F. J. Walker, and C. H. Ahn. Adv. Mat. Interfaces, 7:1901772, 2020.

${ }^{272}$ K. Nashimoto, D. K. Fork, and T. H. Geballe. Appl. Phys. Lett., 60:1199, 1992.

${ }^{273}$ B. Meunier, R. Bachelet, G. Grenet, C. Botella, P. Regreny, L. Largeau, J. Penuelas, and G. Saint-Girons. Journal of Crystal Growth, 433:139, 2016.

${ }^{274}$ St. v. Náray-Szabó. Naturwissenschaften, 31:466, 1943.

${ }^{275}$ H. D. Megaw. Proc. Phys. Soc., 58:133, 1946.

${ }^{276}$ G. Shirane, S. Hoshino, and K. Suzuki. J. Phys. Soc. Jpn., 5:453, 1950.

${ }^{277}$ G. Shirane and S. Hoshino. J. Phys. Soc. Jpn., 6:265, 1951.

${ }^{278}$ N. Sicron, B. Ravel, Y. Yacoby, E. A. Stern, F. Dogan, and J. J. Rehr. Phys. Rev. B, 50:13168, 1994.

${ }^{279}$ J. F. Scott and C. A. Paz de Araujo. Science, 246:1400, 1989.

${ }^{280}$ S. A. Mabud and A. M. Glazer. Journal of Applied Crystallography, 12:49, 1979.

${ }^{281}$ A. M. Glazer and S. A. Mabud. Acta Crystallographica Section B Structural Crystallography and Crystal Chemistry, 34:1065, 1978.

${ }^{282}$ R. E. Cohen. Nature, 358:136, 1992.

${ }^{283}$ W. L. Warren, J. Robertson, D. Dimos, B. A. Tuttle, G. E. Pike, and D. A. Payne. Phys. Rev. B, 53:3080, 1996.

${ }^{284}$ Y. Kuroiwa, S. Aoyagi, A. Sawada, J. Harada, E. Nishibori, M. Takata, and M. Sakata. Phys. Rev. Lett., 87:217601, 2001. 
Epitaxial ferroelectric interfacial devices

${ }^{285}$ X. Gu, N. Izyumskaya, V. Avrutin, H. Morkoç, T. D. Kang, and H. Lee. Appl. Phys. Lett., 89:122912, 2006.

${ }^{286}$ Y.-M. Wu and J.-T. Lo. Japanese J. Appl. Phys., 37:5645, 1998.

${ }^{287}$ V. R. Palkar, S. C. Purandare, and R. Pinto. Journal of Physics D: Applied Physics, 32:R1, 1999.

${ }^{288}$ M. Okuyama and Y. Hamakawa. International Journal of Engineering Science, 29:391, 1991.

${ }^{289} \mathrm{Li}$ Sun, Yan-Feng Chen, Peng Li, Tao Yu, Jian-Xie Chen, and Nai-Ben Ming. Journal of Physics: Condensed Matter, 8:10185, 1996.

${ }^{290}$ G. Rispens and B. Noheda. Integrated Ferroelectrics, 92:30, 2007.

${ }^{291}$ K. Wasa, Y. Haneda, T. Sato, H. Adachi, I. Kanno, D. G. Schlom, S. Trolier-McKinstry, Q. Gang, and C.-B. Eom. Step-flow growth of perovskite PbTiO 3 thin films epitaxially grown on a miscut SrTiO 3 substrate. In Davor Pavuna and Ivan Bozovic, editors, Superconducting and Related Oxides: Physics and Nanoengineering III, volume 3481, page $182,1998$.

${ }^{292}$ S. Venkatesan, A. Vlooswijk, B. J. Kooi, A. Morelli, G. Palasantzas, J. T. M. De Hosson, and B. Noheda. Phys. Rev. B, 78:104112, 2008.

${ }^{293}$ Y.-F. Chen, T. Yu, J.-X. Chen, L. Shun, P. Li, and N.-B. Ming. Appl. Phys. Lett., 66:148, 1995.

${ }^{294}$ Y. Gao, G. Bai, K.L. Merkle, Y. Shi, H.L.M. Chang, Z. Shen, and D.J. Lam. Journal of Materials Research, 8:145, 1993.

${ }^{295}$ H. Fujisawa, Y. Seioh, M. Kume, and M. Shimizu. Japanese J. Appl. Phys., 47:7505, 2008.

${ }^{296}$ B. Jaffe, Jr. W. R. Cook, and H. Jaffe. Piezoelectric ceramics. Academic Press, London, 1971.

${ }^{297}$ D. I. Woodward, J. Knudsen, and I. M. Reaney. Phys. Rev. B, 72:104110, 2005.

${ }^{298}$ B. Noheda, D. E. Cox, G. Shirane, J. A. Gonzalo, L. E. Cross, and S.-E. Park. Appl. Phys. Lett., 74:2059, 1999.

${ }^{299}$ B. Noheda, J. A. Gonzalo, L. E. Cross, R. Guo, S.-E. Park, D. E. Cox, and G. Shirane. Phys. Rev. B, 61:8687, 2000.

${ }^{300}$ V. R. Cooper, I. Grinberg, N. R. Martin, and A. M. Rappe. AIP Conference Proceedings, $626: 26,2002$. 
Epitaxial ferroelectric interfacial devices

${ }^{301}$ N. A. Pertsev, V. G. Kukhar, H. Kohlstedt, and R. Waser. Phys. Rev. B, 67:054107, 2003.

${ }^{302}$ B. Noheda and D. E. Cox. Phase Transitions, 79:5, 2006.

${ }^{303}$ N. Zhang, H. Yokota, A. M. Glazer, Z. Ren, D. A. Keen, D. S. Keeble, P. A. Thomas, and Z.-G. Ye. Nature Communications, 5:5231, 2014.

${ }^{304}$ T. R. Shrout and S. J. Zhang. J. Electroceram., 19:111, 2007.

${ }^{305}$ R. S. Solanki, S. K. Mishra, A. Senyshyn, I. Ishii, C. Moriyoshi, T. Suzuki, Y. Kuroiwa, and D. Pandey. Phys. Rev. B, 86:174117, 2012.

${ }^{306}$ F. Cordero, F. Trequattrini, F. Craciun, and C. Galassi. Phys. Rev. B, 87:094108, 2013.

${ }^{307}$ M. J. Haun, E. Furman, S. J. Jang, H. A. McKinstry, and L. E. Cross. J. Appl. Phys., 62:3331, 1987.

${ }^{308}$ C. M. Foster, G.-R. Bai, R. Csencsits, J. Vetrone, R. Jammy, L. A. Wills, E. Carr, and Jun Amano. J. Appl. Phys., 81:2349, 1997.

${ }^{309}$ T. Oikawa, M. Aratani, K. Saito, and H. Funakubo. Journal of Crystal Growth, 237-239:455, 2002.

${ }^{310}$ S. Yokoyama, H. Funakubo, H. Morioka, K. Saito, T. Yamada, and M. Ishikawa. Ferroelectrics, 389:10, 2009.

${ }^{311}$ N. Izyumskaya, V. Avrutin, X. Gu, B. Xiao, S. Chevtchenko, J.-G. Yoon, H. Morkoç, L. Zhou, and D. J. Smith. Appl. Phys. Lett., 91:182906, 2007.

${ }^{312}$ I. Vrejoiu, D. Hesse, and M. Alexe. Single crystalline pzt films and the impact of extended structural defects on the ferroelectric properties. In Z.-G. Ye, editor, Handbook of Advanced Dielectric, Piezoelectric and Ferroelectric Materials, page 695. Woodhead Publishing Limited, Cambridge, 2008.

${ }^{313}$ S. Gariglio, N. Stucki, J.-M. Triscone, and G. Triscone. Appl. Phys. Lett., 90:202905, 2007.

${ }^{314}$ P.-E. Janolin. J. Mater. Sci., 44:5025, 2009.

${ }^{315}$ C. A. F. Vaz, J. Hoffman, Y. Segal, J. W. Reiner, R. D. Grober, Z. Zhang, C. H. Ahn, and F. J. Walker. Phys. Rev. Lett., 104:127202, 2010.

${ }^{316}$ C. A. F. Vaz, Y. Segal, J. Hoffman, F. J. Walker, and C. H. Ahn. J. Vac. Sci. Technol. B, 28:C5A6, 2010.

${ }^{317}$ H. J. A. Molegraaf, J. Hoffman, C. A. F. Vaz, S. Gariglio, D. van der Marel, C. H. Ahn, and J.-M. Triscone. Adv. Mater., 21:3470, 2009. 
Epitaxial ferroelectric interfacial devices

${ }^{318}$ S. Zhang, F. Li, J. Luo, R. Sahul, and T. R. Shrout. IEEE Transactions on Ultrasonics, Ferroelectrics, and Frequency Control, 60:1572, 2013.

${ }^{319}$ Shujun Zhang and Fei Li. J. Appl. Phys., 111:031301, 2012.

${ }^{320}$ S. Zhang, F. Li, X. Jiang, J. Kim, J. Luo, and X. Geng. Progress in Materials Science, $68: 1,2015$.

${ }^{321}$ P. Miao, Y. Zhao, N. Luo, D. Zhao, A. Chen, Z. Sun, M. Guo, M. Zhu, H. Zhang, and Q. Li. Scientific Reports, 6:19965, 2016.

${ }^{322}$ U. Gabor, D. Vengust, Z. Samardžija, A. Matavž, V. Bobnar, D. Suvorov, and M. Spreitzer. Applied Surface Science, 513:145787, 2020.

${ }^{323}$ M. Tyunina, J. Levoska, and S. Leppävuori. Journal of Materials Science: Materials in Electronics, 14:369, 2003.

${ }^{324}$ V. Nagarajan, C. S. Ganpule, B. Nagaraj, S. Aggarwal, S. P. Alpay, A. L. Roytburd, E. D. Williams, and R. Ramesh. Appl. Phys. Lett., 75:4183, 1999.

${ }^{325}$ M. Tyunina, J. Levoska, P.-E. Janolin, and A. Dejneka. Phys. Rev. B, 87:224107, 2013. ${ }^{326}$ V. Nagarajan, S. P. Alpay, C. S. Ganpule, B. K. Nagaraj, S. Aggarwal, E. D. Williams, A. L. Roytburd, and R. Ramesh. Appl. Phys. Lett., 77:438, 2000.

${ }^{327}$ M. D. Nguyen, C. T. Q. Nguyen, H. N. Vu, and G. Rijnders. Journal of the European Ceramic Society, 38:95, 2018.

${ }^{328}$ X. Wang, L. Zhang, X. Hao, and S. An. Materials Research Bulletin, 65:73, 2015.

${ }^{329}$ M. D. Biegalski, D. H. Kim, S. Choudhury, L. Q. Chen, H. M. Christen, and K. Dorr. Appl. Phys. Lett., 98(14):142902, 2011.

${ }^{330}$ M. D. Biegalski, K. Dorr, D. H. Kim, and H. M. Christen. Appl. Phys. Lett., 96:151903, 2010.

${ }^{331}$ C. Thiele, K. Dörr, O. Bilani, J. Rödel, and L. Schultz. Phys. Rev. B, 75:054408, 2007.

${ }^{332}$ A. D. Rata, A. Herklotz, K. Nenkov, L. Schultz, and K. Dörr. Phys. Rev. Lett., 100:076401, 2008.

${ }^{333}$ M. Buzzi, R. V. Chopdekar, J. L. Hockel, A. Bur, T. Wu, N. Pilet, P. Warnicke, G. P. Carman, L. J. Heyderman, and F. Nolting. Phys. Rev. Lett., 111:027204, 2013.

${ }^{334}$ S. Finizio, M. Foerster, M. Buzzi, B. Krüger, M. Jourdan, C. A. F. Vaz, J. Hockel, T. Miyawaki, A. Tkach, S. Valencia, F. Kronast, G. P. Carman, F. Nolting, and M. Kläui. Phys. Rev. Appl., 1:021001, 2014. 
Epitaxial ferroelectric interfacial devices

${ }^{335}$ K. Y. Yun, D. Ricinschi, T. Kanashima, M. Noda, and M. Okuyama. Jpn. J. Appl. Phys., 43:L647, 2004.

${ }^{336}$ K. Y. Yun, M. Noda, M. Okuyama, H. Saeki, H. Tabata, and K. Saito. J. Appl. Phys., 96:3399, 2004.

${ }^{337}$ V. V. Shvartsman, W. Kleemann, R. Haumont, and J. Kreisel. Appl. Phys. Lett., 90:172115, 2007.

${ }^{338}$ D. Lebeugle, D. Colson, A. Forget, M. Viret, P. Bonville, J. F. Marucco, and S. Fusil. Phys. Rev. B, 76:024116, 2007.

${ }^{339}$ J. B. Neaton, C. Ederer, U. V. Waghmare, N. A. Spaldin, and K. M. Rabe. Phys. Rev. B, 71:014113, 2005

${ }^{340}$ P. Ravindran, R. Vidya, A. Kjekshus, H. Fjellvåg, and O. Eriksson. Phys. Rev. B, 74:224412, 2006.

${ }^{341}$ H. Zheng, F. Straub, Q. Zhan, P.-L. Yang, W.-K. Hsieh, F. Zavaliche, Y.-H. Chu, U. Dahmen, and R. Ramesh. Adv. Mater., 18:2747, 2006.

${ }^{342}$ D. Lebeugle, D. Colson, A. Forget, M. Viret, A. M. Bataille, and A. Gusakov. Phys. Rev. Lett., 100:227602, 2008.

${ }^{343}$ H. Béa, M. Bibes, F. Ott, B. Dupeé, X.-H. Zhu, S. Petit, S. Fusil, C. Deranlot, K. Bouzehouane, and A. Barthélémy. Phys. Rev. Lett., 100:017204, 2008.

${ }^{344}$ M. Bibes and A. Barthélémy. Nature Materials, 7:425, 2008.

${ }^{345}$ S. M. Wu, S. A. Cybart, P. Yu, M. D. Rossell, J. X. Zhang, R. Ramesh, and R. C. Dynes. Nature Mater., 9:756, 2010.

${ }^{346}$ J. T. Heron, M. Trassin, K. Ashraf, M. Gajek, Q. He, S.Y. Yang, D. E. Nikonov, Y-H. Chu, S. Salahuddin, and R. Ramesh. Phys. Rev. Lett., 107:217202, 2011.

${ }^{347}$ S. M. Wu, S. A. Cybart, D. Yi, J. M. Parker, R. Ramesh, and R. C. Dynes. Phys. Rev. Lett., 110:067202, 2013.

${ }^{348}$ J. T. Heron, D. G. Schlom, and R. Ramesh. Appl. Phys. Rev., 1:021303, 2014.

${ }^{349}$ W. Saenrang, B. A. Davidson, F. Maccherozzi, J. P. Podkaminer, J. Irwin, R. D. Johnson, J. W. Freeland, J. Íñiguez, J. L. Schad, K. Reierson, J. C. Frederick, C. A. F. Vaz, L. Howald, T. H. Kim, S. Ryu, M. v. Veenendaal, P. G. Radaelli, S. S. Dhesi, M. S. Rzchowski, and C. B. Eom. Nature Communications, 8:1583, 2017.

${ }^{350}$ B. Xu, B. Dupé, C. Xu, H. Xiang, and L. Bellaiche. Phys. Rev. B, 98:184420, 2018. 
Epitaxial ferroelectric interfacial devices

${ }^{351}$ B. Xu, S. Meyer, M. J. Verstraete, L. Bellaiche, and B. Dupé. Phys. Rev. B, 103:214423, 2021.

${ }^{352}$ I. Sosnowska, T. Peterlin-Neumaier, and E. Steichele. J. Phys. C: Sol. State Phys., $15: 4835,1982$.

${ }^{353}$ S. Picozzi and C. Ederer. J. Phys.: Condens. Matter, 21:303201, 2009.

${ }^{354}$ I. Gross, W. Akhtar, V. Garcia, L. J. Martínez, S. Chouaieb, K. Garcia, C. Carrétéro, A. Barthélémy, P. Appel, P. Maletinsky, J.-V. Kim, J. Y. Chauleau, N. Jaouen, M. Viret, M. Bibes, S. Fusil, and V. Jacques. Nature, 549:252, 2017.

${ }^{355}$ C. Ederer and N. A. Spaldin. Phys. Rev. B, 71:060401, 2005.

${ }^{356}$ M. Ramazanoglu, M. Laver, W. Ratcliff II, S. M. Watson, W. C. Chen, A. Jackson, K. Kothapalli, S. Lee, S.-W. Cheong, and V. Kiryukhin. Phys. Rev. Lett., 107:207206, 2011.

${ }^{357}$ J. Jeong, M. Duc Le, P. Bourges, S. Petit, S. Furukawa, S.-A. Kim, S. Lee, S-W. Cheong, and J.-G. Park. Phys. Rev. Lett., 113:107202, 2014.

${ }^{358}$ J.-G. Park, M. D. Le, J. Jeong, and S. Lee. J. Phys.: Condens. Matter, 26:433202, 2014. ${ }^{359}$ F. Bai, J. Wang, M. Wuttig, J. Li, N. Wang, A. P. Pyatakov, A. K. Zvezdin, L. E. Cross, and D. Viehland. Appl. Phys. Lett., 86:032511, 2005.

${ }^{360}$ H. Béa, M. Bibes, S. Petit, J. Kreisel, and A. Barthélémy. Phil. Mag. Lett., 87:165, 2007. ${ }^{361}$ D. Sando, A. Agbelele, D. Rahmedov, J. Liu, P. Rovillain, C. Toulouse, I. C. Infante, A. P. Pyatakov, S. Fusil, E. Jacquet, C. Carrétéro, C. Deranlot, S. Lisenkov, D. Wang, J. M. Le Breton, M. Cazayous, A. Sacuto, J. Juraszek, A. K. Zvezdin, L. Bellaiche, B. Dkhil, A. Barthélémy, and M. Bibes. Nature Materials, 12(7):641, 2013.

${ }^{362}$ D. Sando, F. Appert, Bin Xu, O. Paull, S. R. Burns, C. Carrétéro, B. Dupé, Y. Gallais, A. Sacuto, M. Cazayous, B. Dkhil, J. M. Le Breton, A. Barthélémy, M. Bibes, L. Bellaiche, V. Nagarajan, and J. Juraszek. Appl. Phys. Rev., 6:041404, 2019.

${ }^{363}$ A. Haykal, J. Fischer, W. Akhtar, J.-Y. Chauleau, D. Sando, A. Finco, F. Godel, Y. A. Birkhölzer, C. Carrétéro, N. Jaouen, M. Bibes, M. Viret, S. Fusil, V. Jacques, and V. Garcia. Nature Comms., 11:1704, 2020.

${ }^{364}$ J. Wang, J. B. Neaton, H. Zheng, V. Nagarajan, S. B. Ogale, B. Liu, D. Viehland, V. Vaithyanathan, D. G. Schlom, U. V. Waghmare, N. A. Spaldin, K. M. Rabe, M. Wuttig, and R. Ramesh. Science, 299:1719, 2003. 
Epitaxial ferroelectric interfacial devices

${ }^{365}$ F. Zavaliche, S. Y. Yang, T. Zhao, Y. H. Chu, M. P. Cruz, C. B. Eom, and R. Ramesh. Phase Transitions, 79:991, 2006.

${ }^{366}$ A. M. Kadomtseva, Yu. F. Popov, A. P. Pyatakov, G. P. Vorob'ev, and D. Viehland. Phase Transitions, 79:1019, 2006.

${ }^{367}$ R. Ramesh, F. Zavaliche, Y. H. Chu, L. W. Martin, S. Y. Yang, M. P. Cruz, M. Barry, K. Lee, P. Yang, and Q. Zhan. Phil. Mag. Lett., 87:155, 2007.

${ }^{368}$ S. Geprägs, M. Opel, S. T. B. Goennenwein, and R. Gross. Phil. Mag. Lett., 87:141, 2007.

${ }^{369}$ G. Catalan and J. F. Scott. Adv. Mater., 21:2463, 2009.

${ }^{370}$ D. Sando, A. Barthélémy, and M. Bibes. J. Phys.: Condens. Matter, 26:473201, 2014.

${ }^{371}$ D. Sando, Bin Xu, L. Bellaiche, and V. Nagarajan. Appl. Phys. Rev., 3:011106, 2016.

${ }^{372}$ D. Ricinschi, K.-Y. Yun, and M. Okuyama. J. Phys.: Condens. Matter, 18:L97, 2006.

${ }^{373}$ H. Yamada, V. Garcia, S. Fusil, S. Boyn, M. Marinova, A. Gloter, S. Xavier, J. Grollier, E. Jacquet, C. Carrétéro, C. Deranlot, M. Bibes, and A. Barthélémy. ACS Nano, 7:5385, 2013.

${ }^{374}$ J. Zhou, M. Trassin, Q. He, N. Tamura, M. Kunz, C. Cheng, J. Zhang, W.-I. Liang, J. Seidel, C.-L. Hsin, and J. Wu. J. Appl. Phys., 112:064102, 2012.

${ }^{375}$ K. Chu, B. K. Jang, J. H. Sung, Y. A. Shin, E. S. Lee, K. Song, J. H. Lee, C. S. Woo, S. J. Kim, S. Y. Choi, T. Y. Koo, Y. H. Kim, S. H. Oh, M. H. Jo, and C. H. Yang. Nature Nanotechnology, 10:972, 2015.

${ }^{376}$ R. C. Haislmaier, N. J. Podraza, S. Denev, A. Melville, D. G. Schlom, and V. Gopalan. Appl. Phys. Lett., 103:031906, 2013.

${ }^{377}$ T. S. Böscke, J. Müller, D. Bräuhaus, U. Schröder, and U. Böttger. Appl. Phys. Lett., 99:102903, 2011.

${ }^{378}$ M. H. Park, T. Schenk, C. M. Fancher, E. D. Grimley, C. Zhou, C. Richter, J. M. LeBeau, J. L. Jones, T. Mikolajick, and U. Schroeder. J. Mater. Chem. C, 5:4677, 2017.

${ }^{379}$ Z. Fan, J. Chen, and J. Wang. Journal of Advanced Dielectrics, 6:1630003, 2016.

${ }^{380}$ X. Tian, S. Shibayama, T. Nishimura, T. Yajima, S. Migita, and A. Toriumi. Appl. Phys. Lett., 112:102902, 2018.

${ }^{381}$ R. Materlik, C. Künneth, and A. Kersch. J. Appl. Phys., 117:134109, 2015.

${ }^{382}$ M. H. Park, Y. H. Lee, H. J. Kim, T. Schenk, W. Lee, K. D. Kim, F. P. G. Fengler, T. Mikolajick, U. Schroeder, and C. S. Hwang. Nanoscale, 9:9973, 2017. 
Epitaxial ferroelectric interfacial devices

${ }^{383}$ S. Liu and B. M. Hanrahan. Physical Review Materials, 3:054404, 2019.

${ }^{384}$ M. Dogan, N. Gong, T.-P. Ma, and S. Ismail-Beigi. Phys. Chem. Chem.Phys., 21:12150, 2019 .

${ }^{385}$ S. J. Kim, J. Mohan, S. R. Summerfelt, and J. Kim. JOM, 71:246, 2019.

${ }^{386}$ M. H. Park, Y. H. Lee, T. Mikolajick, U. Schroeder, and C. S. Hwang. Adv. Electron. Mater., 5:1800522, 2019.

${ }^{387}$ T. Shimizu, K. Katayama, T. Kiguchi, A. Akama, T. J. Konno, and H. Funakubo. Appl. Phys. Lett., 107:032910, 2015.

${ }^{388}$ K. Katayama, T. Shimizu, O. Sakata, T. Shiraishi, S. Nakamura, T. Kiguchi, A. Akama, T. J. Konno, H. Uchida, and H. Funakubo. Appl. Phys. Lett., 109:112901, 2016.

${ }^{389}$ T. Shimizu, K. Katayama, T. Kiguchi, A. Akama, T. J. Konno, O. Sakata, and H. Funakubo. Scientific Reports, 6:32931, 2016.

${ }^{390}$ T. Mimura, T. Shimizu, H. Uchida, O. Sakata, and H. Funakubo. Appl. Phys. Lett., 113:102901, 2018.

${ }^{391}$ T. Shimizu. Journal of the Ceramic Society of Japan, 126:667, 2018.

${ }^{392}$ K. Lee, T. Y. Lee, S. M. Yang, D. H. Lee, J. Park, and S. C. Chae. Appl. Phys. Lett., 112:202901, 2018 .

${ }^{393}$ T. Li, M. Ye, Z. Sun, N. Zhang, W. Zhang, S. Inguva, C. Xie, L. Chen, Y. Wang, S. Ke, and H. Huang. ACS Appl. Mater. Interfaces, 11:4139, 2019.

${ }^{394}$ J. Lyu, I. Fina, J. Fontcuberta, and F. Sánchez. ACS Appl. Mater. Interfaces, 11:6224, 2019.

${ }^{395}$ I. Fina and F. Sánchez. ACS Appl. Electron. Mater., 3:1530, 2021.

${ }^{396}$ X. Li, C. Li, Z. Xu, Y. Li, Y. Yang, H. Hu, Z. Jiang, J. Wang, J. Ren, C. Zheng, C. Lu, and Z. Wen. Phys. Status Solidi RRL, 15:2000481, 2021.

${ }^{397}$ T. Song, R. Bachelet, G. Saint-Girons, N. Dix, I. Fina, and F. Sánchez. J. Mater. Chem. C, XX:XXXX, 2021.

${ }^{398}$ J. Cao, S. Shi, Y. Zhu, and J. Chen. Phys. Status Solidi RRL, 15:2100025, 2021.

${ }^{399}$ Y. Qi, S. Singh, C. Lau, F.-T. Huang, X. Xu, F. J. Walker, C. H. Ahn, S.-W. Cheong, and K. M. Rabe. Phys. Rev. Lett., 125:257603, 2020.

${ }^{400}$ T. Mikolajick, S. Slesazeck, M. H. Park, and U. Schroeder. MRS Bulletin, 43:340, 2018.

${ }^{401}$ M. H. Park, Y. H. Lee, T. Mikolajick, U. Schroeder, and C. S. Hwang. MRS Communications, 8:795, 2018 
Epitaxial ferroelectric interfacial devices

${ }^{402}$ M. Dragoman, M. Aldrigo, D. Dragoman, S. Iordanescu, A. Dinescu, and M. Modreanu. Phys. Status Solidi RRL, 15:2000521, 2021.

${ }^{403}$ M. Dogan and S. Ismail-Beigi. J. Phys. Chem. C, 123:14350, 2019.

${ }^{404}$ M. Dogan, S. Fernandez-Peña, L. Kornblum, Y. Jia, D. P. Kumah, J. W. Reiner, Z. Krivokapic, A. M. Kolpak, S. Ismail-Beigi, C. H. Ahn, and F. J. Walker. Nano Lett., 18:241, 2018.

${ }^{405}$ K. M. Rabe. Current Opinion in Solid State and Materials Science, 9:122, 2005.

${ }^{406}$ J. H. Haeni, P. Irvin, W. Chang, R. Uecker, P. Reiche, Y. L. Li, S. Choudhury, W. Tian, M. E. Hawley, B. Craigo, A. K. Tagantsev, X. Q. Pan, S. K. Streiffer, L. Q. Chen, S. W. Kirchoefer, J. Levy, and D. G. Schlom. Nature, 430:758, 2004.

${ }^{407}$ A. Antons, J. B. Neaton, K. M. Rabe, and D. Vanderbilt. Phys. Rev. B, 71:024102, 2005. ${ }^{408}$ Y. S. Kim, D. J. Kim, T. H. Kim, T. W. Noh, J. S. Choi, B. H. Park, and J.-G. Yoon. Appl. Phys. Lett., 91:042908, 2007.

${ }^{409}$ S. Aggarwal and R. Ramesh. Annu. Rev. Mater. Sci., 28:463, 1998.

${ }^{410}$ J. F. Scott. Jpn. J. Appl. Phys., 38:2272, 1999.

${ }^{411}$ J. F. Scott. Ferroelectric Memories. Springer-Verlag, Berlin, 2000.

${ }^{412}$ L. Pintilie, C. Ghica, C. M. Teodorescu, I. Pintilie, C. Chirila, I. Pasuk, L. Trupina, L. Hrib, A. G. Boni, N. G. Apostol, L. E. Abramiuc, R. Negrea, M. Stefan, and D. Ghica. Scientific Reports, 5:14974, 2015.

${ }^{413}$ S. V. Kalinin, Y. Kim, D. D. Fong, and A. N. Morozovska. Rep. Prog. Phys., 81:036502, 2018.

${ }^{414}$ L. C. Tănase, L. E. Abramiuc, D. G. Popescu, A.-M. Trandafir, N. G. Apostol, I. C. Bucur, L. Hrib, L. Pintilie, I. Pasuk, L. Trupină, and C. M. Teodorescu. Phys. Rev. Appl., 10:034020, 2018.

${ }^{415}$ P. Yu, W. Luo, D. Yi, J. X. Zhang, M. D. Rossell, L. You C.-H. Yang and, G. SinghBhalla, S. Y. Yang, Q. He, Q. M. Ramasse, R. Erni, L. W. Martin, Y. H. Chu, S. T. Pantelides, S. J. Pennycook, and R. Ramesh. PNAS, 109:9710, 2012.

${ }^{416}$ J. Chen, H. Lu, H.-J. Liu, Y.-H. Chu, S. Dunn, K. Ostrikov, A. Gruverman, and N. Valanoor. Appl. Phys. Lett., 102:182904, 2013.

${ }^{417}$ F. C. Frank and J. H. van der Merwe. Proc. Roy. Soc. A, 198:205, 1949.

${ }^{418}$ J. H. van der Merwe. J. Appl. Phys., 34:117, 1963.

${ }^{419}$ J. W. Matthews and A. E. Blakeslee. J. Crystal Growth, 27:118, 1974. 
Epitaxial ferroelectric interfacial devices

${ }^{420}$ L. B. Freund. MRS Bulletin, 17:52, 1992.

${ }^{421}$ J. Y. Tsao. Materials fundamentals of molecular beam epitaxy. Academic Press, Inc., 1993.

${ }^{422}$ R. Beanland, D. J. Dunstan, and P. J. Goodhew. Adv. Phys., 45:87, 1996.

${ }^{423}$ F. K. LeGoues, M. Copel, and R. Tromp. Phys. Rev. Lett., 63:1826, 1989.

${ }^{424}$ D. J. Srolovitz. Acta mettal., 37:621, 1989.

${ }^{425}$ B. J. Spencer, P. W. Voorhees, and S. H. Davis. Phys. Rev. Lett., 67:3696, 1991.

${ }^{426}$ S. S. Iyer and F. K. LeGoues. J. Appl. Phys., 65:4693, 1989.

${ }^{427}$ P. S. S. R. Krishnan, A. N. Morozovska, E. A. Eliseev, Q. M. Ramasse, D. Kepaptsoglou, W.-I. Liang, Y.-H. Chu, P. Munroe, and V. Nagarajan. J. Appl. Phys., 115:054103, 2014.

${ }^{428}$ D. H. Kim, H. N. Lee, M. D. Biegalski, and H. M. Christen. Appl. Phys. Lett., 92:012911, 2008 .

${ }^{429}$ A. Gruverman, B. J. Rodriguez, A. I. Kingon, R. J. Nemanich, A. K. Tagantsev, J. S. Cross, and M. Tsukada. Appl. Phys. Lett., 83:728, 2003.

${ }^{430}$ G. Catalan, L. J. Sinnamon, and J. M. Gregg. J. Phys.: Condens. Matter, 16:2253, 2004.

${ }^{431}$ G. Catalan, B. Noheda, J. McAneney, L. J. Sinnamon, and J. M. Gregg. Phys. Rev. B, 72:020102(R), 2005.

${ }^{432}$ P. V. Yudin and A. K. Tagantsev. Nanotechnology, 24:432001, 2013.

${ }^{433}$ B. C. Jeon, D. Lee, M. H. Lee, S. M. Yang, S. C. Chae, T. K. Song, S. D. Bu, J.-S. Chung, J.-G. Yoon, and T. W. Noh. Adv. Mater., 25:5643, 2013.

${ }^{434}$ R. Guo, L. Shen, H. Wang, Z. Lim, W. Lu, P. Yang, Ariando, A. Gruverman, T. Venkatesan, Y. P. Feng, and J. Chen. Adv. Mater. Interfaces, 3:1600737, 2016.

${ }^{435}$ J. L. Blok, D. H. A. Blank, G. Rijnders, K. M. Rabe, and D. Vanderbilt. Phys. Rev. B, 84:205413, 2011

${ }^{436}$ C. Daumont, W. Ren, I. C. Infante, S. Lisenkov, J. Allibe, C. Carrétéro, S. Fusil, E. Jacquet, T. Bouvet, F. Bouamrane, S. Prosandeev, G. Geneste, B. Dkhil, L. Bellaiche, A. Barthélémy, and M. Bibes. J. Phys.: Condens. Matter, 24:162202, 2012.

${ }^{437}$ C. Ederer and N. A. Spaldin. Phys. Rev. B, 71:224103, 2005.

${ }^{438}$ H. N. Lee, S. M. Nakhmanson, M. F. Chisholm, H. M. Christen, K. M. Rabe, and D. Vanderbilt. Phys. Rev. Lett., 98:217602, 2007.

${ }^{439}$ J. X. Zhang, Q. He, M. Trassin, W. Luo, D. Yi, M. D. Rossell, P. Yu, L. You, C. H. Wang, C. Y. Kuo, J. T. Heron, Z. Hu, R. J. Zeches, H. J. Lin, A. Tanaka, C. T. Chen, 
Epitaxial ferroelectric interfacial devices

L. H. Tjeng, Y.-H. Chu, and R. Ramesh. Phys. Rev. Lett., 107:147602, 2011.

${ }^{440}$ Alison J. Hatt, Nicola A. Spaldin, and Claude Ederer. Phys. Rev. B, 81:054109, 2010.

${ }^{441}$ Netanela Cohen and Oswaldo Diéguez. Israel Journal of Chemistry, 60:833-841, 2020.

${ }^{442}$ R. W. Whatmore, R. Clarke, and A. M. Glazer. J. Phys. C: Solid State Phys., 11:3089, 1978 .

${ }^{443}$ N. A. Pertsev, A. G. Zembilgotov, and A. K. Tagantsev. Phys. Rev. Lett., 80:1988, 1998.

${ }^{444}$ M. M. Saad, P. Baxter, R. M. Bowman, J. M. Gregg, F. D. Morrison, and J. F. Scott. J. Phys.: Condens. Matter, 16:L451, 2004.

${ }^{445}$ Y. L. Li and L. Q. Chen. Appl. Phys. Lett., 88:072905, 2006.

${ }^{446}$ I. C. Infante, S. Lisenkov, B. Dupé, M. Bibes, S. Fusil, E. Jacquet, G. Geneste, S. Petit, A. Courtial, J. Juraszek, L. Bellaiche, A. Barthélémy, and B. Dkhil. Phys. Rev. Lett., 105:057601, 2010.

${ }^{447}$ S. P. Alpay, I. B. Misirlioglu, V. Nagarajan, and R. Ramesh. Appl. Phys. Lett., 85:2044, 2004.

${ }^{448}$ I. B. Misirlioglu, A. L. Vasiliev, M. Aindow, and S. P. Alpay. Integrated Ferroelectrics, 71:67, 2005 .

${ }^{449}$ I. B. Misirlioglu, G. Akcay, and S. P. Alpay. Integrated Ferroelectrics, 83:67, 2006.

${ }^{450}$ C. L. Jia, S. B. Mi, K. Urban, I. Vrejoiu, M. Alexe, and D. Hesse. Phys. Rev. Lett., 102:117601, 2009.

${ }^{451}$ A. Yu. Emelyanov and N. A. Pertsev. Phys. Rev. B, 68:214103, 2003.

${ }^{452}$ M.-W. Chu, I. Szafraniak, R. Scholz, C. Harnagea, D. Hesse, M. Alexe, and U. Gösele. Nature Materials, 3:87, 2004.

${ }^{453}$ V. Nagarajan, C. L. Jia, H. Kohlstedt, R. Waser, I. B. Misirlioglu, S. P. Alpay, and R. Ramesh. Appl. Phys. Lett., 86:192910, 2005.

${ }^{454}$ I. B. Misirlioglu, A. L. Vasiliev, S. P. Alpay, M. Aindow, and R. Ramesh. J. Mater. Sci., 41:697, 2006.

${ }^{455}$ I. B. Misirlioglu, S. P. Alpay, M. Aindow, and V. Nagarajan. Appl. Phys. Lett., 88:102906, 2006 .

${ }^{456}$ I. Vrejoiu, G. Le Rhun, N. D. Zakharov, D. Hesse, L. Pintilie, and M. Alexe. Philosophical Magazine, 86:4477, 2006.

${ }^{457}$ W. Pompe, X. Gong, Z. Suo, and J. S. Speck. J. Appl. Phys., 74:6012, 1993.

${ }^{458}$ J. S. Speck and W. Pompe. J. Appl. Phys., 76:466, 1994. 
Epitaxial ferroelectric interfacial devices

${ }^{459}$ J. S. Speck, A. C. Daykin, A. Seifert, A. E. Romanov, and W. Pompe. J. Appl. Phys., 78:1696, 1995.

${ }^{460}$ J. S. Speck, A. Seifert, W. Pompe, and R. Ramesh. J. Appl. Phys., 76:477, 1994.

${ }^{461}$ T. Zhao, A. Scholl, F. Zavaliche, K. Lee, M. Barry, A. Doran, M. P. Cruz, Y. H. Chu, C. Ederer, N. A. Spaldin, R. R. Das, D. M. Kim, S. H. Baek, C. B. Eom, and R. Ramesh. Nature Materials, 5:823, 2006.

${ }^{462}$ D. D. Fong, A. M. Kolpak, J. A. Eastman, S. K. Streiffer, P. H. Fuoss, G. B. Stephenson, C. Thompson, D. M. Kim, K. J. Choi, C. B. Eom, I. Grinberg, and A. M. Rappe. Phys. Rev. Lett., 96:127601, 2006.

${ }^{463}$ J. E. Spanier, A. M. Kolpak, J. J. Urban, I. Grinberg, L. Ouyang, W. S. Yun, A. M. Rappe, and H. Park. Nano Lett., 6:735, 2006.

${ }^{464}$ P. Aguado-Puente and J. Junquera. Phys. Rev. Lett., 100:177601, 2008.

${ }^{465}$ A. M. Bratkovsky and A. P. Levanyuk. Journal of Computational and Theoretical Nanoscience, 6:465, 2009.

${ }^{466}$ J. Junquera and P. Ghosez. Nature, 422:506, 2003.

${ }^{467}$ N. Sai, A. M. Kolpak, and A. M. Rappe. Phys. Rev. B, 72:020101, 2005.

${ }^{468}$ S. P. Alpay, I. B. Misirlioglu, and V. Nagarajan. Appl. Phys. Lett., 90:236101, 2007.

${ }^{469}$ N. D. Mermim and H. Wagner. Phys. Rev. Lett., 17:1133, 1966.

${ }^{470}$ Y. S. Kim, J. Y. Jo, D. J. Kim, Y. J. Chang, J. H. Lee, T. W. Noh, T. K. Song, J.-G. Yoon, J.-S. Chung, S. I. Baik, Y.-W. Kim, and C. U. Jung. Appl. Phys. Lett., 88:072909, 2006.

${ }^{471}$ Y. J. Shin, Y. Kim, S. J. Kang, H. H. Nahm, P. Murugavel, J. R. Kim, M. R. Cho, L. Wang, S. M. Yang, J. G. Yoon, J. S. Chung, M. Kim, H. Zhou, S. H. Chang, and T. W. Noh. Adv. Mater., 29:1602795, 2017.

${ }^{472}$ S. R. Lee, L. Baasandorj, J. W. Chang, I. W. Hwang, J. R. Kim, J.-G. Kim, K.-T. Ko, S. B. Shim, M. W. Choi, M. Y., C.-H. Yang, J. Kim, and J. Song. Nano Lett., 19:2243, 2019.

${ }^{473}$ T. Tybell, C. H. Ahn, and J.-M. Triscone. Appl. Phys. Lett., 75:856, 1999.

${ }^{474}$ P. Maksymovych, M. Huijben, M. Pan, S. Jesse, N. Balke, Y.-H. Chu, H. J. Chang, A. Y. Borisevich, A. P. Baddorf, G. Rijnders, D. H. A. Blank, R. Ramesh, and S. V. Kalinin. Phys. Rev. B, 85:014119, 2012. 
Epitaxial ferroelectric interfacial devices

${ }^{475}$ M. Tyunina, L. D. Yao, D. Chvostova, T. Kocourek, M. Jelinek, A. Dejneka, and S van Dijken. New Journal of Physics, 17:043048, 2015.

${ }^{476}$ L. Pintilie and M. Alexe. J. Appl. Phys., 98:124103, 2005.

${ }^{477}$ L. Pintilie, I. Boerasu, M. J. M. Gomes, T. Zhao, R. Ramesh, and M. Alexe. J. Appl. Phys., 98:124104, 2005.

${ }^{478}$ A. Klein. J. Am. Ceram. Soc., 99:369, 2016.

${ }^{479}$ M. Stengel, P. Aguado-Puente, N. A. Spaldin, and J. Junquera. Phys. Rev. B, 83:235112, 2011.

${ }^{480}$ F. Chen, R. Schafranek, W. Wu, and A. Klein. J. Phys. D: Appl. Phys., 44:255301, 2011.

${ }^{481}$ F. Chen and A. Klein. Phys. Rev. B, 86:094105, 2012.

${ }^{482}$ D. Goren, N. Amir, and Y. Nemirovsky. J. Appl. Phys., 71:318, 1992.

${ }^{483}$ L. Pintilie, V. Stancu, L. Trupina, and I. Pintilie. Phys. Rev. B, 82:085319, 2010.

${ }^{484}$ H. T. Yi, T. Choi, S. G. Choi, Y. S. Oh, and S.-W. Cheong. Adv. Mater., 23:3403, 2011. ${ }^{485}$ X. Liu, Y. Wang, J. D. Burton, and E. Y. Tsymbal. Phys. Rev. B, 88:165139, 2013.

${ }^{486}$ J. E. Rault, G. Agnus, T. Maroutian, V. Pillard, Ph. Lecoeur, G. Niu, B. Vilquin, M. G. Silly, A. Bendounan, F. Sirotti, and N. Barrett. Phys. Rev. B, 87:155146, 2013.

${ }^{487}$ L. Pintilie. Charge transport in ferroelectric thin films. In M. Lallart, editor, Ferroelectrics - Physical Effects, page 101. InTech, Rijeka (Croatia), 2011.

${ }^{488}$ G. Catalan, J. Seidel, R. Ramesh, and J. F. Scott. Rev. Mod. Phys., 84:119, 2012.

${ }^{489}$ N. A. Spaldin. Novel functionalities at oxide (multi)ferroic domain walls. ${ }^{71}$, page 32.

${ }^{490}$ J. Seidel, L. W. Martin, Q. He, Q. Zhan, Y.-H. Chu, A. Rother, M. E. Hawkridge, P. Maksymovych, P. Yu, M. Gajek, N. Balke, S. V. Kalinin, S. Gemming, F. Wang, G. Catalan, J. F. Scott, N. A. Spaldin, J. Orenstein, and R. Ramesh. Nature Materials, 8:229, 2009 .

${ }^{491}$ J. Seidel, P. Maksymovych, Y. Batra, A. Katan, S.-Y. Yang, Q. He, A. P. Baddorf, S.V. Kalinin, C.-H. Yang, J.-C. Yang, Y.-H. Chu, E. K. H. Salje, H. Wormeester, M. Salmeron, and R. Ramesh. Phys. Rev. Lett., 105:197603, 2010.

${ }^{492}$ S. Farokhipoor and B. Noheda. Phys. Rev. Lett., 107:127601, 2011.

${ }^{493}$ J. Guyonnet, I. Gaponenko, S. Gariglio, and P. Paruch. Adv. Mater., 23:5377, 2011.

${ }^{494}$ D. Meier, J. Seidel, A. Cano, K. Delaney, Y. Kumagai, M. Mostovoy, N. A. Spaldin, R. Ramesh, and M. Fiebig. Nature Materials, 11:284, 2012. 
Epitaxial ferroelectric interfacial devices

${ }^{495}$ T. Sluka, A. K. Tagantsev, P. Bednyakov, and N. Setter. Nature Communications, 4:1808, 2013.

${ }^{496}$ Y.-M. Kim, A. Morozovska, E. Eliseev, M. P. Oxley, R. Mishra, S. M. Selbach, T. Grande, S. T. Pantelides, S. V. Kalinin, and A. Y. Borisevich. Nature Materials, 13:1019, 2014.

${ }^{497}$ A. Zenkevich, M. Minnekaev, Yu. Matveyev, Yu. Lebedinskii, K. Bulakh, A. Chouprik, A. Baturin, K. Maksimova, S. Thiess, and W. Drube. Appl. Phys. Lett., 102:062907, 2013.

${ }^{498}$ C.-L. Wu, P.-W. Lee, Y.-C. Chen, Lo-Y. Chang, C.-H. Chen, C.-W. Liang, P. Yu, Q. He, R. Ramesh, and Y.-H. Chu. Phys. Rev. B, 83:020103(R), 2011.

${ }^{499}$ B. C. Huang, Y. T. Chen, Y. P. Chiu, Y. C. Huang, J. C. Yang, and Y. C. Chen. Appl. Phys. Lett., 100:122903, 2012.

${ }^{500}$ G. Gerra, A. K. Tagantsev, N. Setter, and K. Parlinski. Phys. Rev. Lett., 96(10):107603, 2006.

${ }^{501}$ M. F. Chisholm, W. Luo, M. P. Oxley, S. T. Pantelides, and H. N. Lee. Phys. Rev. Lett., 105:197602, 2010.

${ }^{502}$ I. I. Naumov, L. Bellaiche, and H. Fu. Nature, 432:737, 2004.

${ }^{503}$ J. F. Scott. J. Phys.: Condens. Matter, 18:R361, 2006.

${ }^{504}$ V. M. Fridkin. Physics-Uspekhi, 49:193, 2006.

${ }^{505}$ M. J. Polking, A. P. Alivisatos, and R. Ramesh. MRS Communications, 5:27, 2015.

${ }^{506}$ C. G. Duan, R. F. Sabirianov, W. N. Mei, S. S. Jaswal, and E. Y. Tsymbal. Nano Lett., 6(3):483, 2006.

${ }^{507}$ L. Bégon-Lours, V. Rouco, Q. Qiao, A. Sander, M. A. Roldán, R. Bernard, J. Trastoy, A. Crassous, E. Jacquet, K. Bouzehouane, M. Bibes, J. Santamaría, A. Barthélémy, M. Varela, and J. E. Villegas. Phys. Rev. Mater., 2:084405, 2018.

${ }^{508}$ C. Noguera. J. Phys.: Condens. Matter, 12:R367, 2000.

${ }^{509}$ N. Nakagawa, H. Y. Hwang, and D. A. Muller. Nature Materials, 5:204, 2006.

${ }^{510}$ J. Goniakowski, F. Finocchi, and C. Noguera. Rep. Prog. Phys., 71:016501, 2008.

${ }^{511}$ S. Hong, S. M. Nakhmanson, and D. D. Fong. Rep. Prog. Phys., 79:076501, 2016.

${ }^{512}$ X. H. Liu, Y. Wang, P. V. Lukashev, J. D. Burton, and E. Y. Tsymbal. Phys. Rev. B, 85:125407, 2012.

${ }^{513}$ G. Gerra, A. K. Tagantsev, and N. Setter. Phys. Rev. Lett., 98(20):207601, 2007. 
Epitaxial ferroelectric interfacial devices

${ }^{514}$ H. Lu, X. Liu, J. D. Burton, C. W. Bark, Y. Wang, Y. Zhang, D. J. Kim, A. Stamm, P. Lukashev, D. A. Felker, C. M. Folkman, P. Gao, M. S. Rzchowski, X. Q. Pan, C. B. Eom, E. Y. Tsymbal, and A. Gruverman. Adv. Mater., 24:1209, 2012.

${ }^{515}$ R.D. Shannon and C.T. Prewitt. Acta Cryst. B, 25:925, 1969.

${ }^{516}$ R.D. Shannon and C.T. Prewitt. Acta Cryst. B, 26:1046, 1970.

${ }^{517}$ A. K. Tagantsev, I. Stolichnov, E. L. Colla, and N. Setter. J. Appl. Phys., 90:1387, 2001. ${ }^{518}$ X. J. Lou. J. Appl. Phys., 105:024101, 2009.

${ }^{519}$ P.-E. Janolin, F. Le Marrec, J. Chevreul, and B. Dkhil. Appl. Phys. Lett., 90:192910, 2007.

${ }^{520}$ P.-E. Janolin, B. Fraisse, F. Le Marrec, and B. Dkhil. Appl. Phys. Lett., 90:212904, 2007. ${ }^{521}$ S. Venkatesan, B. J. Kooi, J. T. M. De Hosson, A. H. G. Vlooswijk, and B. Noheda. J. Appl. Phys., 102:104105, 2007.

${ }^{522}$ S. Stemmer, S. K. Streiffer, F. Ernst, and M. Rühle. phys. stat. sol. (a), 147:135, 1995. ${ }^{523}$ B. J. Spencer, S. H. Davis, and P. W. Voorhees. Phys. Rev. B, 47:9760, 1993.

${ }^{524}$ B. J. Spencer, P. W. Voorhees, and S. H. Davis. J. Appl. Phys., 73:4955, 1993.

${ }^{525}$ A. L. Roitburd. phys. stat. sol. (a), 37:329, 1976.

${ }^{526}$ N. A. Pertsev and A. Yu. Emel'yanov. Phys. Solid State, 39:109, 1997.

${ }^{527}$ Y. Kamimura, K. Edagawa, and S. Takeuchi. Acta Materialia, 61:294, 2013.

${ }^{528}$ C. A. F. Vaz, J. Hoffman, A.-B. Posadas, and C. H. Ahn. Appl. Phys. Lett., 94:022504, 2009.

${ }^{529}$ D. Ferré, P. Carrez, and P. Cordier. Phys. Rev. B, 77:014106, 2008.

${ }^{530}$ D. Ferré, P. Carrez, and P. Cordier. Phys. Chem. Minerals, 36:233, 2009.

${ }^{531}$ A. M. Walker, P. Carrez, and P. Cordier. Mineralogical Magazine, 74:381, 2010.

${ }^{532}$ H. P. Sun, W. Tian, X. Q. Pan, J. H. Haeni, and D. G. Schlom. Appl. Phys. Lett., 84:3298, 2004.

${ }^{533}$ W. Wunderlich, M. Fujimoto, and H. Ohsato. Materials Science and Engineering A, 309-310:148, 2001.

${ }^{534}$ M. Kawai, D. Kan, S. Isojima, H. Kurata, S. Isoda, S. Kimura, O. Sakata, and Y. Shimakawa. Mater. Res. Soc. Symp. Proc., 1034:1034-K10-04, 2008.

${ }^{535}$ C. L. Canedy, Hao Li, S. P. Alpay, L. Salamanca-Riba, A. L. Roytburd, and R. Ramesh. Appl. Phys. Lett., 77:1695, 2000. 
Epitaxial ferroelectric interfacial devices

${ }^{536}$ V. Metlenko, A. H. H. Ramadan, F. Gunkel, H. Du, H. Schraknepper, S. Hoffmann-Eifert,

R. Dittmann, R. Waser, and R. A. De Souza. Nanoscale, 6:12864, 2014.

${ }^{537}$ S. P. Waldow and R. A. De Souza. ACS Appl. Mater. Interfaces, 8:12246, 2016.

${ }^{538}$ E. Navickas, Y. Chen, Q. Lu, W. Wallisch, T. M. Huber, J. Bernardi, M. St" oger-Pollach,

G. Friedbacher, H. Hutter, B. Yildiz, and J. Fleig. ACS Nano, 11:11475, 2017.

${ }^{539}$ M. Arredondo, Q. M. Ramasse, M. Weyland, R. Mahjoub, I. Vrejoiu, D. Hesse, N. D.

Browning, M. Alexe, P. Munroe, and V. Nagarajan. Adv. Mater., 22:2430, 2010.

${ }^{540}$ F. Sandiumenge. Front. Mater., 6:13, 2019.

${ }^{541}$ D. Lee and T. W. Noh. Phil. Trans. R. Soc. A, 370:4944, 2012.

${ }^{542}$ X. Yu, L. Wu, B. Zhang, H. Zhou, Y. Dong, X. Wu, R. Kou, P. Yang, J. Chen, C.-J.

Sun, Y. Zhu, , and G. M. Chow. Phys. Rev. B, 25:104405, 2019.

${ }^{543}$ C. M. Foster, W. Pompe, A. C. Daykin, and J. S. Speck. J. Appl. Phys., 79:1405, 1996.

${ }^{544}$ L. Feigl, I. B. Misirlioglu, I. Vrejoiu, M. Alexe, and D. Hesse. J. Appl. Phys., 105:061607, 2009.

${ }^{545}$ P. E. Hopkins, C. Adamo, L. Ye, B. D. Huey, S. R. Lee, D. G. Schlom, and J. F. Ihlefeld. Appl. Phys. Lett., 102:121903, 2013.

${ }^{546}$ F. Riesz. Surface Science Letters, 292:L817, 1993.

${ }^{547}$ F. Riesz. Czechoslovak Journal of Physics, 44:131, 1994.

${ }^{548}$ H. W. Jang, D. Ortiz, S.-H. Baek, C. M. Folkman, R. R. Das, P. Shafer, Y. Chen, C. T. Nelson, X.g Pan, R. Ramesh, and C.-B. Eom. Adv. Mater., 21:817, 2009.

${ }^{549}$ J. E. Giencke, C. M. Folkman, S.-H. Baek, and C.-B. Eom. Current Opinion in Solid State and Materials Science, 18:39, 2014.

${ }^{550}$ M. Mietschke, S. Oswald, S. Fähler, L. Schultz, and R. Hühne. Thin Solid Films, 589:792, 2015.

${ }^{551}$ P. Chekhonina, M. Mietschke, D. Pohl, F. Schmidt, S. Fähler, W. Skrotzki, K. Nielsch, and R. Hühne. Materials Characterization, 129:234, 2017.

${ }^{552}$ H. Fujisawa, H. Yane, Y. Hiki, S. Nakashima, and M. Shimizu. Journal of the Korean Physical Society, 59:2560, 2011.

${ }^{553}$ Y. Lin and C. L. Chen. J. Mater. Sci., 44:5274, 2009.

${ }^{554}$ T. H. Kim, S. H. Baek, S. Y. Jang, S. M. Yang, S. H. Chang, T. K. Song, J.-G. Yoon, C. B. Eom, J.-S. Chung, and T. W. Noh. Appl. Phys. Lett., 98:022904, 2011.

${ }^{555}$ M. J. Zhuo, Y. L. Zhu, and X. L. Ma. Philosophical Magazine Letters, 86:469, 2006. 
Epitaxial ferroelectric interfacial devices

${ }^{556}$ D. A. McQuarrie. Statistical Mechanics. University Science Books, Sausalito, 2000.

${ }^{557}$ N.-H. Chan, R. K. Sharma, and D. M. Smyth. J. Am. Ceram. Soc., 64:556, 1981.

${ }^{558}$ H. Yamada and G. R. Miller. Journal of Solid State Chemistry, 6:169, 1973.

${ }^{559}$ M. Martin. Diffusion in oxides. In Diffusion in Condensed Matter, page 209. SpringerVerlag, Berlin, 2005.

${ }^{560}$ R. A. De Souza. Adv. Funct. Mater., 25:6326, 2015.

${ }^{561}$ F. A. Kröger and H. J. Vink. Solid State Physics, 3:307, 1956.

${ }^{562}$ D. M. Smyth. Current Opinion in Solid State and Materials Science, 1:692, 1996.

${ }^{563}$ M. V. Raymond and D. M. Smyth. J. Phys. Chem. Solids, 57:1507, 1996.

${ }^{564}$ D. M. Smyth, E. K. Chang, and D. H. Liu. Phase Transitions, 58:57, 1996.

${ }^{565}$ N.-H. Chan and D. M. Smyth. J. Electrochem. Soc., 123:1584, 1976.

${ }^{566}$ G. A. Boni, C. F. Chirila, V. Stancu, L. Amarande, I. Pasuk, L. Trupina, C. M. Istrate, C. Radu, A. Tomulescu, S. Neatu, I. Pintilie, and L. Pintilie. Nanomaterials, 11:1177, 2021.

${ }^{567}$ M. Siebenhofer, T. Huber, W. Artner, J. Fleig, and M. Kubicek. Acta Materialia, 203:116461, 2021.

${ }^{568}$ D. M. Smyth. Ferroelectrics, 151:115, 1994.

${ }^{569}$ M. Morgenbesser, A. Viernstein, A. Schmid, C. Herzig, N. Bodenmueller, M. Kubicek, S. Taibl, G. Bismashofer, J. Stahn, C. A. F. Vaz, M. Doebeli, F. Baiutti, J. de Dios Sirvent, M. O. Liedke, M. Butterling, M. Kaminski, M. Tolkiehn, V. Vonk, A. Stierle, A. Wagner, A. Tarancon, A. Limbeck, and J. Fleig. Unravelling the origin of ultra-low conductivity in doped $\mathrm{SrTiO}_{3}$ thin films: Sr vacancies as the key driver for fermi level pinning. 2021. unpublished.

${ }^{570}$ A. Viernstein, G. Bimashofer, M. Kubicek, M. Morgenbesser, T. Huber, E. Ellmeyer, M. Siebenhofer, C. A. F. Vaz, J. Stahn, and J. Fleig. Mechanism of photo-ionic stoichiometry changes in $\mathrm{SrTiO}_{3}$. 2021. unpublished.

${ }^{571}$ R.-A. Eichel, H. Kungl, and P. Jakes. Materials Technology, 28:241, 2013.

${ }^{572}$ K. Shimoyama, M. Kiyohara, K. Kubo, A. Uedono, and K. Yamabe. J. Appl. Phys., 92:4625, 2002.

${ }^{573}$ T. Tanaka, K. Matsunaga, Y. Ikuhara, and T. Yamamoto. Phys, Rev. B, 68:205213, 2003. 
Epitaxial ferroelectric interfacial devices

${ }^{574}$ S. R. Basu, L. W. Martin, Y. H. Chu, M. Gajek, R. Ramesh, R. C. Rai, X. Xu, and J. L. Musfeldt. Appl. Phys. Lett., 92:091905, 2008.

${ }^{575}$ Y. Feng, J. Wu, Q. Chi, W. Li, Y. Yu, and W. Fei. Chem. Rev., 120:1710, 2020.

${ }^{576}$ S. Pöykkö and D. J. Chadi. Appl. Phys. Lett., 76:499, 2000.

${ }^{577}$ M. Kotiuga et al. PNAS, 116:21992, 2019.

${ }^{578}$ T. H. Etsell and S. N. Flengas. Chem. Rev., 70:339, 1970.

${ }^{579}$ C. López-Gándara, F. M. Ramos, and A. Cirera. Journal of Sensors, 2009:258489, 2009.

${ }^{580}$ X.-D. Zhang, J.-J. Li, and X. Guo. Rev. Sci. Instrum., 86:115103, 2015.

${ }^{581}$ C. Ahamer, A. K. Opitz, G. M. Rupp, and J. Fleig. Journal of The Electrochemical Society, 164:F790, 2017.

${ }^{582}$ D. Ricci, G. Bano, G. Pacchioni, and F. Illas. Phys, Rev. B, 68:224105, 2003.

${ }^{583}$ W. L. Warren, K. Vanheusden, D. Dimos, G. E. Pike, and B. A. Tuttle. J. Am. Ceram. Soc., 79:536, 1996.

${ }^{584}$ F. Cordero. Phys, Rev. B, 76:172106, 2007.

${ }^{585}$ D. D. Cuong, B. Lee, K. M. Choi, H.-S. Ahn, S. Han, and J. Lee. Phys. Rev. Lett., 98:115503, 2007.

${ }^{586}$ D. J. Wouters, G. J. Willems, and H. E. Maes. Microelectronic Engineering, 29:249, 1995.

${ }^{587}$ W. L. Warren, G. E. Pike, B. A. Tuttle, and D. Dimos. Appl. Phys. Lett., 70:2010, 1997. ${ }^{588}$ C. Ahn, A. Cavalleri, A. Georges, S. Ismail-Beigi, A. J. Millis, and J.-M. Triscone. Nature Materials, 2021.

${ }^{589}$ H. Vo, S. Zhang, W. Wang, and G. Galli. J. Chem. Phys., 154:174704, 2021.

${ }^{590}$ M. Imada, A. Fujimori, and Y. Tokura. Rev. Mod. Phys., 70:1039, 1998.

${ }^{591}$ A. Moreo, S. Yunoki, and E. Dagotto. Science, 283:2034, 1999.

${ }^{592}$ Y. Tokura and Y. Tomioka. J. Magn. Magn. Mater., 200:1, 1999.

${ }^{593}$ E. Dagotto, T. Hotta, and A. Moreo. Phys. Rep., 344:1, 2001.

${ }^{594}$ Y. Tokura. Rep. Prog. Phys., 69:797, 2006.

${ }^{595}$ A. Zunger and O. I. Malyi. Chemical Reviews, 121:3031, 2021.

${ }^{596}$ X. Hong, J.B. Yau, J. D. Hoffman, C. H. Ahn, Y. Bason, and L. Klein. Phys. Rev. B, 74:174406, 2006.

${ }^{597}$ S. Dasgupta, B. Das, M. Knapp, R. A. Brand, H. Ehrenberg, R. Kruk, and H. Hahn. Adv. Mater., 26:4639, 2014. 
Epitaxial ferroelectric interfacial devices

${ }^{598}$ U. Bauer, L. Yao, A. J. Tan, P. Agrawal, S. Emori, H. L. Tuller, S. van Dijken, and G. S. D. Beach. Nature Materials, 14:174, 2015.

${ }^{599}$ D. A. Gilbert, A. J. Grutter, E. Arenholz, K. Liu, B.J. Kirby, J. A. Borchers, and B. B. Maranville. Nature Communications, 7:12264, 2016.

${ }^{600}$ J. A. Van Orman and K. L. Crispin. Reviews in Mineralogy and Geochemistry, 72:757, 2010.

${ }^{601}$ A. van der Ven, J. Bhattacharya, and A. A. Belak. Accounts of Chemical Research, 46:1216, 2013.

${ }^{602}$ D. Cooper, C. Baeumer, N. Bernier, A. Marchewka, C. La Torre, R. E. Dunin-Borkowski, S. Menzel, R. Waser, and R. Dittmann. Adv. Mater., 29:1700212, 2017.

${ }^{603}$ S. Mathews, R. Ramesh, T. Venkatesan, and J. Benedetto. Science, 276:238, 1997.

${ }^{604}$ T. Venkatesan, D. C. Kundaliya, T. Wu, and S. B. Ogale. Phil. Mag. Lett., 87:279, 2007.

${ }^{605}$ M. Weisheit, S. Fähler, A. Marty, Y. Souche, C. Poinsignon, and D. Givord. Science, 315:349, 2007.

${ }^{606}$ A. Molinari, H. Hahn, and R. Kruk. Adv. Mater., 31:1806662, 2019.

${ }^{607}$ S. L. Miller and P. J. McWhorter. J. Appl. Phys., 72:5999, 1992.

${ }^{608}$ Y.-G. Park, T. Kanki, H.-Y. Lee, H. Tanaka, and T. Kawai. Solid-State Electronics, 47:2221, 2003.

${ }^{609}$ H. Ishiwara. FeFET and ferroelectric random access memories. In E. Y. Tsymbal, E. R. A. Dagotto, C.-B. Eom, and R. Ramesh, editors, Multifunctional oxide heterostructures, chapter 12, page 340. Oxford Scholarship Online, 2012.

${ }^{610}$ C. Visani, A. Crassous, and J. E. Villegas. Eur. Phys. J. Special Topics, 222:1241, 2013.

${ }^{611}$ J. H. Ngai, F. J. Walker, and C. H. Ahn. Annu. Rev. Mater. Res., 44:1, 2014.

${ }^{612}$ J. Bardeen, L. N. Cooper, and J. R. Schrieffer. Phys. Rev., 106:162, 1957.

${ }^{613}$ J. Bardeen, L. N. Cooper, and J. R. Schrieffer. Phys. Rev., 108:1175, 1957.

${ }^{614}$ M. R. Norman. Science, 332:196, 2011.

${ }^{615}$ B. Keimer, S. A. Kivelson, M. R. Norman, S. Uchida, and J. Zaanen. Nature, 518:179, 2015.

${ }^{616}$ I. Božović, J. Wua, X. He, and A. T. Bollinger. Physica C: Superconductivity and its applications, 558:30, 2019.

${ }^{617}$ III R. E. Glover and M. D. Sherrill. Phys. Rev. Lett., 5:248, 1960.

${ }^{618}$ H. L. Stadler. Phys. Rev. Lett., 14:979, 1965. 
Epitaxial ferroelectric interfacial devices

${ }^{619}$ T. G. Berlincourt. Physics Letters, 29A:308, 1969.

${ }^{620}$ A. Cassinese, G. M. De Luca, A. Prigiobbo, M. Salluzzo, and R. Vaglio. Appl. Phys. Lett., 84:3933, 2004.

${ }^{621}$ W. J. Gallagher. IEEE Transactions on Magnetics, MAG-21:709, 1985.

${ }^{622}$ T. Nishino, M. Hatano, H. Hasegawa, F. Murai, T. Kure, A. Hiraiwa, K. Yagi, and U. Kawabe. IEEE Electron Device Letters, 10:61, 1989.

${ }^{623}$ A. W. Kleinsasser. Superconducting field-effect devices. In H. Weinstock and R. W. Ralston, editors, The New Superconducting Electronics, page 249. Kluwer Academic Publishers, 1993.

${ }^{624}$ J. Mannhart. Supercond. Sci. Technol., 9:49, 1996.

${ }^{625}$ F. Paolucci, G. De Simoni, P. Solinas, E. Strambini, C. Puglia, N. Ligato, and F. Giazotto. AVS Quantum Sci., 1:016501, 2019.

${ }^{626}$ N. W. Ashcroft and N. D. Mermin. Solid state physics. Saunder College, Philadelphia, 1976.

${ }^{627}$ O. Madelung. Introduction to solid-state theory. Springer-Verlag, Berlin, 1978.

${ }^{628}$ J. Mannhart, J. G. Bednorz, K. A. Müller, D. G. Schlom, and J. Ströbel. Journal of Alloys and Compounds, 195:519, 1993.

${ }^{629}$ K. A. Parendo, K. H. S. B. Tan, A. Bhattacharya, M. Eblen-Zayas, N. E. Staley, and A. M. Goldman. Phys. Rev. Lett., 94:197004, 2005.

${ }^{630}$ G. De Simoni, F. Paolucci, P. Solinas, E. Strambini, and F. Giazotto. Nature Nanotechnology, 13:802, 2018.

${ }^{631}$ J. Mannhart, J. Ströbel, J. G. Bednorz, and Ch. Gerber. Appl. Phys. Lett., 62:630, 1993.

${ }^{632}$ X. X. Xi. Journal of Superconductivity, 7:137, 1994.

${ }^{633}$ J. G. Bednorz and K. A. Müller. Z. Phys. B, 64:189, 1986.

${ }^{634}$ T. Timusk and B. Statt. Rep. Prog. Phys., 62:61, 1999.

${ }^{635}$ A. Mourachkine. High-Temperature Superconductivity in Cuprates. Number 125 in Fundamental Theories of Physics. Kluwer Academic Publishers, New York, 2002.

${ }^{636}$ R. L. Meng, L. Beauvais, X. N. Zhang, Z. J. Huang, Y. Y. Sun, Y. Y. Xue, and C. W. Chu. Physica C, 216:21, 1993.

${ }^{637}$ A. T. Fjory, A. F. Hebard, R. H. Eick, P. M. Mankiewich, R. E. Howard, and M. L. O’Malley. Phys. Rev. Lett., 65:3441, 1990. 
Epitaxial ferroelectric interfacial devices

${ }^{638}$ J. Mannhart, D. G. Schlom, J. G. Bednorz, and K. A. Müller. Phys. Rev. Lett., 67:2099, 1991.

${ }^{639}$ A. Walkenhorst, C. Doughty, X. X. Xi, Qi Li, C. J. Lobb, S. N. Mao, and T. Venkatesan. Phys. Rev. Lett., 69:2709, 1996.

${ }^{640}$ X. X. Xi, C. Doughty, A. Walkenhorst, C. Kwon, Q. Li, and T. Venkatesan. Phys. Rev. Lett., 68:1240, 1996.

${ }^{641}$ V. C. Matijasevic, S. Bogers, N. Y. Chen, H. M. Appelboom, P. Hadley, and J. E. Mooij. Physlca C, 235-240:2097, 1994.

${ }^{642}$ T. Frey, J. Mannhart, J. G. Bednorz, and E. J. Williams. Phys. Rev. B, 51:3257, 1995.

${ }^{643}$ V. Talyansky, S. B. Ogale, I. Takeuchi, C. Doughty, and T. Venkatesan. Phys. Rev. B, 53:14575, 1996.

${ }^{644}$ D. Matthey, S. Gariglio, and J.-M. Triscone. Appl. Phys. Lett., 83:3758, 2003.

${ }^{645}$ M. Salluzzo, A. Cassinese, G. M. De Luca, A. Gambardella, A. Prigiobbo, and R. Vaglio. Phys. Rev. B, 70:214528, 2004.

${ }^{646}$ V. V. Lemanov, A. L. Kholkin, and A. B. Sherman. Supercond. Sci. Technol., 6:814, 1993.

${ }^{647}$ V. V. Lemanov, A. L. Kholkin, and A. B. Sherman. Ferroelectrics, 151:109, 1994.

${ }^{648}$ A. Ignatiev, N.-J. Wu, H. Lin, T.-Q. Huang, S. Endicter, X.-Y. Li, and D. Liu. Integrated Ferroelectrics, 10:327, 1995.

${ }^{649}$ C. H. Ahn, S. Gariglio, P. Paruch, T. Tybell, L. Antognazza, and J.-M. Triscone. Science, 284:1152, 1999

${ }^{650}$ R. Aidam, D. Fuchs, and R. Schneider. Physica C, 328:21, 1999.

${ }^{651}$ S. Gariglio, C. H. Ahn, D. Matthey, and J.-M. Triscone. Phys. Rev. Lett., 88:067002, 2002 .

${ }^{652}$ D. Matthey, S. Gariglio, C.H. Ahn, and J.-M. Triscone. Physica C, 372-376:583, 2002.

${ }^{653}$ A. Crassous, R. Bernard, S. Fusil, K. Bouzehouane, D. Le Bourdais, S. Enouz-Vedrenne, J. Briatico, M. Bibes, A. Barthélémy, and J. E. Villegas. Phys. Rev. Lett., 107:247002, 2011.

${ }^{654}$ A. Crassous, R. Bernard, S. Fusil, K. Bouzehouane, J. Briatico, M. Bibes, A. Barthélémy, and J. E. Villegas. J. Appl. Phys., 113:024910, 2013.

${ }^{655}$ J. T. Ye, S. Inoue, K. Kobayashi, Y. Kasahara, H. T. Yuan, H. Shimotani, and Y. Iwasa. Nature Materials, 9:125, 2010. 
Epitaxial ferroelectric interfacial devices

${ }^{656}$ G. Dubuis, A. T. Bollinger, D. Pavuna, and I. Božović. J. Appl. Phys., 111:112632, 2012.

${ }^{657}$ E. Sterpetti, J. Biscaras, A. Erb, and A. Shukla. Nature Communications, 8:2060, 2017.

${ }^{658}$ Y. Zhang and X. Xu. Computational Materials Science, 179:109583, 2020.

${ }^{659}$ J. L. Tallon and N. E. Flower. Physica C, 204:237, 1993.

${ }^{660}$ L. Bégon-Lours, V. Rouco, A. Sander, J. Trastoy, R. Bernard, E. Jacquet, K. Bouzehouane, S. Fusil, V. Garcia, A. Barthélémy, M. Bibes, J. Santamaría, and J. E. Villegas. Phys. Rev. Applied, 7:064015, 2017.

${ }^{661}$ K. S. Takahashi, D. Matthey, D. Jaccard, J.-M. Triscone, K. Shibuya, T. Ohnishi, and M. Lippmaa. Appl. Phys. Lett., 84:1722, 2004.

${ }^{662}$ K. S. Takahashi, M. Gabay, D. Jaccard, K. Shibuya, T. Ohnishi, M. Lippmaa, and J.-M. Triscone. Nature, 441:195, 2006.

${ }^{663}$ T. M. Mishonov. Phys. Rev. Lett., 67:3195, 1991.

${ }^{664}$ A. T. Fjory, A. F. Hebard, R. H. Eick, P. M. Mankiewich, R. E. Howard, and M. L. O’Malley. Phys. Rev. Lett., 67:3196, 1991.

${ }^{665} \mathrm{~S}-$ W. Cheong, S. E. Brown, Z. Fisk, R. S. Kwok, J. D. Thompson, E. Zirngiebl, G. Gruner, D. E. Peterson, G. L. Wells, R. B. Schwarz, and J. R. Cooper. Phys. Rev. B, 36:3913, 1987.

${ }^{666}$ S. Gariglio, C. H. Ahn, D. Matthey, and J.-M. Triscone. Phys. Rev. Lett., 88:067002, 2002 .

${ }^{667}$ A. D. Caviglia, S. Gariglio, N. Reyren, D. Jaccard, T. Schneider, M. Gabay, S. Thiel, G. Hammerl, J. Mannhart, and J.-M. Triscone. Nature, 456:624, 2008.

${ }^{668}$ K. Ueno, S. Nakamura, H. Shimotani, A. Ohtomo, N. Kimura, T. Nojima, H. Aoki, Y. Iwasa, and M. Kawasaki. Nature Materials, 7:855, 2008.

${ }^{669}$ M. Ben Shalom, M. Sachs, D. Rakhmilevitch, A. Palevski, and Y. Dagan. Phys. Rev. Lett., 104:126802, 2010.

${ }^{670}$ A. D. Caviglia, M. Gabay, S. Gariglio, N. Reyren, C. Cancellieri, and J.-M. Triscone. Phys. Rev. Lett., 104:126803, 2010.

${ }^{671}$ G. Singh G. Herranz and, N. Bergeal, A. Jouan, J. Lesueur, J. Gázquez, M. Varela, M. Scigaj, N. Dix, F. Sánchez, and Josep Fontcuberta. Nature Communications, 6:6028, 2015 .

${ }^{672}$ S. Hurand, A. Jouan, C. Feuillet-Palma, G. Singh, J. Biscaras, E. Lesne, N. Reyren, A. Barthélémy, M. Bibes, J. E. Villegas, C. Ulysse, X. Lafosse, M. Pannetier-Lecoeur, 
Epitaxial ferroelectric interfacial devices

S. Caprara, M. Grilli, J. Lesueur, and N. Bergeal. Scientific Reports, 5:12751, 2015.

${ }^{673}$ E. Fillis-Tsirakis, C. Richter, J. Mannhart, and H. Boschker. New J. Phys., 18:013046, 2016.

${ }^{674}$ C. Bell, S. Harashima, Y. Kozuka, M. Kim, B. G. Kim, Y. Hikita, and H.Y. Hwang. Phys. Rev. Lett., 103:226802, 2009.

${ }^{675}$ A. G. Swartz, H. Inoue, T. A. Merz, Y. Hikita, S. Raghu, T. P. Devereaux, S. Johnston, and H. Y. Hwang. PNAS, 115:1475, 2018.

${ }^{676}$ T. Terashima, K. Shimura, Y. Bando, Y. Matsuda, A. Fujiyama, and S. Komiyama. Phys. Rev. Lett., 67:1362, 1991.

${ }^{677}$ J.-M. Triscone and Ø Fischer. Rep. Prog. Phys., 60:1673, 1997.

${ }^{678}$ T. Tybell, C. H. Ahn, and J.-M. Triscone. Appl. Phys. Lett., 72:1454, 1998.

${ }^{679}$ C. H. Ahn, K. M. Rabe, and J.-M. Triscone. Science, 303:488, 2004.

${ }^{680}$ P. W. Anderson. Phys. Rev., 109:1492, 1958.

${ }^{681}$ N. F. Mott. Adv. Phys., 16:49, 1967.

${ }^{682}$ N. F. Mott. Rev. Mod. Phys., 40:677, 1968.

${ }^{683}$ N. F. Mott and Z. Zinamon. Rep. Prog. Phys., 33:881, 1970.

${ }^{684}$ N. F. Mott. Metal-insulator transitions. Taylor \& Francies Ltd, London, 1974.

${ }^{685}$ N. F. Mott. Physics Today, November:42, 1978.

${ }^{686}$ Florian Gebhard. The Mott Metal-Insulator Transition. STMP 137. Springer-Verlag, Berlin Heidelberg, 2000.

${ }^{687}$ Y. Zhou and S. Ramanathan. Critical Reviews in Solid State and Materials Sciences, 38:286, 2013.

${ }^{688}$ S. Salahuddin and S. Datta. Nano Lett., 8:405, 2008.

${ }^{689}$ F. A. McGuire, Y.-C. Lin, K. Price, G. B. Rayner, S. Khandelwal, S. Salahuddin, and A. D. Franklin. Nano Lett., 17:4801, 2017.

${ }^{690}$ J. Íñiguez, P. Zubko, I. Luk'yanchuk, and A. Cano. Nature Reviews Materials, 4:243, 2019.

${ }^{691}$ A. H. Wilson. Proceedings of the Royal Society of London. Series A, 133:458, 1931.

${ }^{692}$ A. S. Balchan and H. G. Drickamer. J. Chem. Phys., 34:1948, 1961.

${ }^{693}$ B. M. Riggleman and H. G. Drickamer. J. Chem. Phys., 37:446, 1962.

${ }^{694}$ B. M. Riggleman and H. G. Drickamer. J. Chem. Phys., 38:2721, 1963.

${ }^{695}$ N. Sakai, K. Takemura, and K. Tsuji. J. Phys. Soc. Jpn., 51:1811, 1982. 
Epitaxial ferroelectric interfacial devices

${ }^{696}$ S. Desgreniers, Y. K. Vohra, and A. L. Ruoff. J. Phys. Chem., 94:1117, 1990.

${ }^{697}$ Y. Ma, A. R. Oganov, and C. W. Glass. Phys. Rev. B, 76:064101, 2007.

${ }^{698}$ G. Weck, S. Desgreniers, B. Loubeyre, and M. Mezouar. Phys. Rev. Lett., 102:255503, 2009.

${ }^{699}$ S. F. Elatresh and S. A. Bonev. Phys. Chem. Chem. Phys., 22:12577, 2020.

${ }^{700}$ T. Ando, A. B. Fowler, and F. Stern. Rev. Mod. Phys., 54:437, 1982.

${ }^{701}$ A. Ohtomo and H. Y. Hwang. Nature, 427:423, 2004.

${ }^{702}$ M. Cuoco and J. van den Brink. What theoretical approaches can provide: a perspective on oxide electronics. ${ }^{167}$, page 6 .

${ }^{703}$ K. Ueno, I. H. Inoue, H. Akoh, M. Kawasaki, Y. Tokura, and H. Takagi. Appl. Phys. Lett., 83:1755, 2003.

${ }^{704}$ K. Shibuya, T. Ohnishi, M. Lippmaa, M. Kawasaki, and H. Koinuma. Appl. Phys. Lett., $85: 425,2004$.

${ }^{705}$ K. Shibuya, T. Ohnishi, T. Uozumi, T. Sato, M. Lippmaa, M. Kawasaki, K. Nakajima, T. Chikyow, and H. Koinuma. Appl. Phys. Lett., 88:212116, 2006.

${ }^{706}$ K. Shibuya, T. Ohnishi, T. Sato, and M. Lippmaa. J. Appl. Phys., 102:083713, 2007.

${ }^{707}$ H. Ohta, Y. Masuoka, R. Asahi, T. Kato, Y. Ikuhara, K. Nomura, and H. Hosono. Appl. Phys. Lett., 95:113505, 2009.

${ }^{708}$ K. Ueno, I. H. Inoue, T. Yamada, H. Akoh, Y. Tokura, and H. Takagic. Appl. Phys. Lett., 84:3726, 2004.

${ }^{709}$ A. Yoshikawa, K. Uchida, K. Koumoto, T. Kato, Y. Ikuhara, and H. Ohta. Appl. Phys. Express, 2:121103, 2009.

${ }^{710}$ M. S. J. Marshall, A. Malashevich, A. S. Disa, M.-G. Han, H. Chen, Y. Zhu, S. IsmailBeigi, F. J. Walker, and C. H. Ahn. Phys. Rev. Applied, 2:051001, 2014.

${ }^{711}$ N. F. Mott and M. Kaveh. Adv. Phys., 34:329, 1985.

${ }^{712}$ M. H. Cohen, H. Fritzsche, and S. R. Ovshinsky. Phys. Rev. Lett., 22:1065, 1969.

${ }^{713}$ T. S. Santos, S. J. May, J. L. Robertson, and A. Bhattacharya. Phys. Rev. B, 80:155114, 2009.

${ }^{714}$ R. E. Peierls. Quantum Theory of Solids. Clarendon Press, Oxford, 1955.

${ }^{715}$ Grüner G. Rev. Mod. Phys., 60:1129, 1988.

${ }^{716}$ J. Varignon, M. Bibes, and A. Zunger. Nature Communications, 10:1658, 2019.

${ }^{717}$ J. Varignon, M. Bibes, and A. Zunger. Phys. Rev. B, 100:035119, 2019. 
Epitaxial ferroelectric interfacial devices

${ }^{718}$ Y. Zhang, J. Furness, R. Zhang, Z. Wang, A. Zunger, and J. Sun. Phys. Rev. B, 102:045112, 2020 .

${ }^{719}$ I. Malyi and A. Zunger. Appl. Phys. Rev., 7:041310, 2020.

${ }^{720}$ J. Zaanen and G. A. Sawatzky. Journal of Solid State Chemistry, 88:8, 1990.

${ }^{721}$ K. van Benthem, C. Elsässer, and R. H. French. J. Appl. Phys., 90:6156, 2001.

${ }^{722}$ Y. Okimoto, T. Katsufuji, Y. Okada, T. Arima, and Y. Tokura. Phys. Rev. B, 51:9581, 1995.

${ }^{723}$ T. Mizokawa and A. Fujimori. Phys. Rev. B, 54:5368, 1996.

${ }^{724}$ S. Miyasaka, Y. Okimoto, M. Iwama, and Y. Tokura. Phys. Rev. B, 68:100406(R), 2003.

${ }^{725}$ T. Arima, Y. Tokura, and J. B. Torrance. Phys. Rev. B, 48:17006, 1993.

${ }^{726}$ A. Kudo, H. Yanagi, H. Hosono, and H. Kawazoe. Appl. Phys. Lett., 73:220, 1998.

${ }^{727}$ K. Tezuka, Y. Hinatsu, A. Nakamura, T. Inami, Y. Shimojo, and Y. Morii. J. Solid State Chem., 141:404, 1998.

${ }^{728}$ M. Eibschütz, S. Shtrikman, and D. Treves. Phys. Rev., 156:562, 1967.

${ }^{729}$ K. Mikhalev, S. Verkhovskii, A. Gerashenko, A. Mirmelstein, V. Bobrovskii, K. Kumagai, Y. Furukawa, T. D'yachkova, and Yu. Zainulin. Phys. Rev. B, 69:132415, 2004.

${ }^{730}$ J. M. Ginder, M. G. Roe, Y. Song, R. P. McCall, J. R. Gaines, E. Ehrenfreund, and A. J. Epstein. Phys. Rev. B, 37:7506, 1988.

${ }^{731}$ A. E. Bocquet, T. Mizokawa, K. Morikawa, A. Fujimori, S. R. Barman, K. Maiti, D. D. Sarma, Y. Tokura, and M. Onoda. Phys. Rev. B, 53:1161, 1996.

${ }^{732}$ T. Saitoh, A. E. Bocquet, T. Mizokawa, and A. Fujimori. Phys. Rev. B, 52:7934, 1995.

${ }^{733}$ A. E. Bocquet, T. Mizokawa, T. Saitoh, H. Namatame, and A. Fujimori. Phys. Rev. B, 46:3771, 1992.

${ }^{734}$ T. Saitoh, T. Mizokawa, A. Fujimori, M. Abbate, Y. Takeda, and M. Takano. Phys. Rev. $B, 55: 4257,1997$.

${ }^{735}$ M. Abbate, G. Zampieri, F. Prado, A. Caneiro, J. M. Gonzalez-Calbet, and M. ValletRegi. Phys. Rev. B, 65:155101, 2002.

${ }^{736}$ B. Keimer, A. Aharony, A. Auerbach, R. J. Birgeneau, A. Cassanho, Y. Endoh, R. W. Erwin, M. A. Kastner, and G. Shirane. Phys. Rev. B, 45:7430, 1992.

${ }^{737}$ A. Fujimori, E. Takayama-Muromachi, Y. Uchida, and B. Okai. Phys. Rev. B, 35:8814, 1987. 
Epitaxial ferroelectric interfacial devices

${ }^{738}$ Z. Shen, J. W. Allen, J. J. Yeh, J. S. Kang, W. Ellis, W. Spicer, I. Lindau, M. B. Maple, Y. D. Dalichaouch, M. S. Torikachvili, J. Z. Sun, and T. H. Geballe. Phys. Rev. B, $36: 8414,1987$.

${ }^{739}$ H. Yasuoka, H. Mazaki, T. Terashima, and Y. Bando. Physica C, 175:192, 1991.

${ }^{740}$ A. Zibold, L. Widder, H. P. Geserich, G. Bräuchle, H. Claus, H. v. Löhneysen, N. Nücker, A. Erb, and G. Müller-Vogt. Physica C, 212:365, 1993.

${ }^{741}$ C. Zener. Phys. Rev., 82:403, 1951.

${ }^{742}$ T. Hatano, Y. Ogimoto, N. Ogawa, M. Nakano, S. Ono, Y. Tomioka, K. Miyano, Y. Iwasa, and Y. Tokura. Scientific Reports, 3:2904, 2013.

${ }^{743}$ R. M. Kusters, J. Singleton, D. A. Keen, R. McGreevy, and W. Hayes. Physica B, 155:362, 1989 .

${ }^{744}$ R. von Helmolt, J. Wecker, B. Holzapfel, L. Schultz, and K. Samwer. Phys. Rev. Lett., 71:2331, 1993.

${ }^{745}$ K. Chahara, T. Ohno, M. Kasai, and Y. Kozono. Appl. Phys. Lett., 63:1990, 1993.

${ }^{746}$ S. Jin, T. H. Tiefel, M. McCormack, R. A. Fastnacht, R. Ramesh, and L. H. Chen. Science, 264:413, 1994.

${ }^{747}$ Y. Tokura, A. Urushibara, Y. Moritomo, T. Arima, A. Asamitsu, G. Kido, and N. Furukawa. J. Phys. Soc. Japan, 63:3931, 1994.

${ }^{748}$ Y. Konishi, Z. Fang, M. Izumi, T. Manako, M. Kasai, H. Kuwahara, M. Kawasaki, K. Terakura, and Y. Tokura. J. Phys. Soc. Japan, 68:3790, 1999.

${ }^{749}$ C. A. F. Vaz, J. A. Moyer, D. Arena, C. H. Ahn, and V. E. Henrich. Phys. Rev. B, 90:024414, 2014.

${ }^{750}$ D. M. Newns, T. Doderer, C. C. Tsuei, W. M. Donath, J. A. Misewich, A. Gupta, B. M. Grossman, A. Schrott, B. A. Scott, P. C. Pattnaik, R. J. von Gutfeld, and J. Z. Sun. Journal of Electroceramics, 4:339, 2000.

${ }^{751}$ J. A. Misewich and A. G. Schrott. Appl. Phys. Lett., 76:3632, 2000.

752Z. Yang, C. Ko, and S. Ramanathan. Annu. Rev. Mater. Res., 41:337, 2011.

${ }^{753}$ Y. Zhou and S. Ramanathan. Proceedings of the IEEE, 103:1289, 2015.

${ }^{754}$ D. M. Newns, J. A. Misewich, C. C. Tsuei, A Gupta, B. A. Scott, and A. Schrott. Appl. Phys. Lett., 73:780, 1998.

${ }^{755}$ M. Nakamura, A. Sawa, H. Sato, H. Akoh, M. Kawasaki, and Y. Tokura. Phys. Rev. B, $75: 155103,2007$. 
Epitaxial ferroelectric interfacial devices

${ }^{756}$ J. Son, S. Rajan, S. Stemmer, and S. J. Allen. J. Appl. Phys., 110:084503, 2011.

${ }^{757}$ T. Hatano, Z. Sheng, M. Nakamura, M. Nakano, M. Kawasaki, Y. Iwasa, and Y. Tokura. Advanced Materials, 26:2874, 2014.

${ }^{758}$ C.H. Ahn, J.-M. Triscone, N. Archibald, M. Decroux, R. H. Hammond, T. H. Geballe, E. Fischer, and M. R. Beasley. Science, 269:373, 1995.

${ }^{759}$ Y. Watanabe. Appl. Phys. Lett., 66:1770, 1995.

${ }^{760}$ X. Hong, A. Posadas, A. Lin, and C. H. Ahn. Phys. Rev. B, 68:134415, 2003.

${ }^{761}$ X. Hong, A. Posadas, and C. H. Ahn. Appl. Phys. Lett., 86:142501, 2005.

${ }^{762}$ A. S. Dhoot, C. Israel, X. Moya, N. D. Mathur, and R. H. Friend. Phys. Rev. Lett., 102:136402, 2009.

${ }^{763}$ J. Hoffman, X. Pan, J. W. Reiner, F. J. Walker, J. P. Han, C. H. Ahn, and T. P. Ma. Advanced Materials, 22:2957, 2010.

${ }^{764}$ J. Hoffman, X. Hong, and C. H. Ahn. Nanotechnology, 22:254014, 2011.

${ }^{765}$ H. Yamada, M. Marinova, P. Altuntas, A. Crassous, L. Bégon-Lours, S. Fusil, E. Jacquet, V. Garcia, K. Bouzehouane, A. Gloter, J. E. Villegas, A. Barthélémy, and M. Bibes. Scientific Reports, 3:2834, 2013.

${ }^{766}$ X. Li, Q. Zhu, L. Vistoli, A. Barthélémy, M. Bibes, S. Fusil, V. Garcia, and A. Gloter. Adv. Mater. Interfaces, page 2000601, 2020.

${ }^{767}$ L. Zhang, X. G. Chen, H. J. Gardner, M. A. Koten, J. E. Shield, and X. Hong. Appl. Phys. Lett., 107:152906, 2015.

${ }^{768}$ X. Chen, X. Zhang, M. A. Koten, H. Chen, Z. Xiao, L. Zhang, J. E. Shield, P. A. Dowben, and X. Hong. Adv. Mater., 29:1701385, 2017.

${ }^{769}$ A. Urushibara, Y. Moritomo, T. Arima, A. Asamitsu, G. Kido, and Y. Tokura. Phys. Rev. B, 51:14103, 1995.

${ }^{770}$ M. Fäth, S. Freisem, A. A. Menovsky, Y. Tomioka, J. Aarts, and J. A. Mydosh. Science, 285:1540, 1999.

${ }^{771}$ A. Biswas, M. Rajeswari, R. C. Srivastava, Y. H. Li, T. Venkatesan, R. L. Greene, and A. J. Millis. Phys. Rev. B, 61:9665, 2000.

${ }^{772}$ J. C. Loudon, N. D. Mathur, and P. A. Midgley. Nature, 420:797, 2002.

${ }^{773}$ T. Wu, S. B. Ogale, J. E. Garrison, B. Nagaraj, A. Biswas, Z. Chen, R. L. Greene, R. Ramesh, T. Venkatesan, and A. J. Millis. Phys. Rev. Lett., 86:5998, 2001. 
Epitaxial ferroelectric interfacial devices

${ }^{774}$ M. Eblen-Zayas, A. Bhattacharya, N. E. Staley, A. L. Kobrinskii, and A. M. Goldman. Phys. Rev. Lett., 94:037204, 2005.

${ }^{775}$ M. L. Medarde. Journal of Physics: Condensed Matter, 9:1679, 1997.

${ }^{776}$ J. B. Torrance, P. Lacorre, A. I. Nazzal, E. J. Ansaldo, and C. Niedermayer. Phys. Rev. B, 45:8209, 1992.

${ }^{777}$ S. J. May, J. W. Kim, J. M. Rondinelli, E. Karapetrova, N. A. Spaldin, A. Bhattacharya, and P. J. Ryan. Phys. Rev. B, 82:014110, 2010.

${ }^{778}$ D. P. Kumah, A. S. Disa, J. H. Ngai, H. Chen, A. Malashevich, J. W. Reiner, S. IsmailBeigi, F. J. Walker, and C. H. Ahn. Adv. Mater., 26:1935, 2014.

${ }^{779}$ D. P. Kumah, A. Malashevich, A. S. Disa, D. A. Arena, F. J. Walker, S. Ismail-Beigi, and C. H. Ahn. Physical Review Applied, 2:054004, 2014.

${ }^{780}$ A. Malashevich, M. S. J. Marshall, C. Visani, A. S. Disa, H. Xu, F. J. Walker, C. H. Ahn, and S. Ismail-Beigi. Nano Lett., 18:573, 2018.

${ }^{781}$ K. I. Kugel' and D. I. Khomskii. Sov. Phys. Usp., 25:231, 1982.

${ }^{782}$ V. I. Anisimov, J. Zaanen, and O. K. Andersen. Phys. Rev. B, 44:943, 1991.

${ }^{783}$ A. I. Liechtenstein, V. I. Anisimov, and J. Zaanen. Phys. Rev. B, 52:R5467, 1995.

${ }^{784}$ Y. Tokura and N. Nagaosa. Science, 288:462, 2000.

${ }^{785}$ D. I. Khomskii and M. V. Mostovoy. J. Phys. A: Math. Gen., 36:9197, 2003.

${ }^{786}$ J. van den Brink, G. Khaliullin, and D. Khomskii. Orbital effects in manganites. In T. Chatterji, editor, Colossal Magnetoresistive Manganites, page 263. Kluwer Academic Publishers, Dordrecht, 2004.

${ }^{787}$ H. A. Jahn and E. Teller. Proceedings of the Royal Society of London. Series A, 161:220, 1937.

${ }^{788}$ Y. Murakami, S. Ishihara, and S. Maekawa. Charge and orbital ordering of manganites observed by resonant x-ray scattering. In T. Chatterji, editor, Colossal Magnetoresistive Manganites, page 383. Kluwer Academic Publishers, Dordrecht, 2004.

${ }^{789}$ T. Miller, M. Gensch, and S. Wall. Physica Scripta, 91:124002, 2016.

${ }^{790}$ M. D. N. Regueiro, M. Altarelli, and C. T. Chen. Phys. Rev. B, 51:629, 1995.

${ }^{791}$ S. Konno, K. Taniguchi, H. Sagayama, and T. Arima. Applied Physics Express, 2:033004, 2009.

${ }^{792}$ J. Varignon, N. C. Bristowe, E. Bousquet, and P. Ghosez. Scientific Reports, 5:15364, 2015. 
Epitaxial ferroelectric interfacial devices

${ }^{793}$ L. Chen, C. Xu, H. Tian, H. Xiang, J. Íñiguez, Y. Yang, and L. Bellaiche. Phys. Rev. Lett., 122:247701, 2019.

${ }^{794}$ C. Aruta, G. Ghiringhelli, A. Tebano, N. G. Boggio, N. B. Brookes, P. G. Medaglia, and G. Balestrino. Phys. Rev. B, 73:235121, 2006.

${ }^{795}$ C. Aruta, C. Adamo, A. Galdi, P. Orgiani, V. Bisogni, N. B. Brookes, J. C. Cezar, P. Thakur, C. A. Perroni, G. De Filippis, V. Cataudella, D. G. Schlom, L. Maritato, and G. Ghiringhelli. Phys. Rev. B, 80:140405(R), 2009.

${ }^{796}$ D. Pesquera, G. Herranz, A. Barla, E. Pellegrin, F. Bondino, E. Magnano, F. Sánchez, and J. Fontcuberta. Nature Communications, 3:1189, 2012.

${ }^{797}$ P. Yu, J.-S. Lee, S. Okamoto, M. D. Rossell, M. Huijben, C.-H. Yang, Q. He, J. X. Zhang, S.Y. Yang, M. J. Lee, Q. M. Ramasse, R. Erni, Y.-H. Chu, D. A. Arena, C.-C.

Kao, L. W. Martin, and R. Ramesh. Phys. Rev. Lett., 105:027201, 2010.

${ }^{798}$ J.-C. Yang, Q. He, P. Yu, and Y.-H. Chu. Annu. Rev. Mater. Res., 45:249, 2015.

${ }^{799}$ H. Chen, Q. Qiao, M. S. J. Marshall, A. B. Georgescu, A. Gulec, P. J. Phillips, R. F. Klie, F. J. Walker, C. H. Ahn, and S. Ismail-Beigi. Nano Letters, 14:4965, 2014.

${ }^{800}$ D. Preziosi, M. Alexe, D. Hesse, and M. Salluzzo. Phys. Rev. Lett., 115:157401, 2015.

${ }^{801}$ B. T. Thole, P. Carra, F. Sette, and G. van der Laan. Phys. Rev. Lett., 68:1943, 1992.

${ }^{802}$ P. Carra, B. T. Thole, M. Altarelli, and X. Wang. Phys. Rev. Lett., 70:694, 1993.

${ }^{803}$ A. Ankudinov and J. J. Rehr. Phys. Rev. B, 51:1282, 1995.

${ }^{804}$ D. J. Huang, H.-T Jeng, C. F. Chang, G. Y. Guo, J. Chen, W. P. Wu, S. C. Chung,

S. G. Shyu, C. C. Wu, H.-J. Lin, and C. T. Chen. Phys. Rev. B, 66:174440, 2002.

${ }^{805}$ A. L. Ankudinov, J. J. Rehr, H. Wende, A. Scherz, and K. Baberschke. Europhysics Letters, 66:441, 2004.

${ }^{806}$ E. J. Verwey. Nature, 144:327, 1939.

${ }^{807}$ E. J. W. Verwey and P. W. Haayman. Physica, 8:979, 1941.

${ }^{808}$ F. de Boer, J. H. van Santen, and E. J. W. Verwey. J. Chem. Phys., 18:1032, 1950.

${ }^{809}$ P. W. Anderson. Phys. Rev., 102:1008, 1956.

${ }^{810}$ K. Yamauchi and S. Picozzi. Phys. Rev. B, 85:085131, 2012.

${ }^{811}$ M. Angst. Phys. Status Solidi RRL, 7:383, 2013.

${ }^{812}$ E. O. Wollan and W. C. Koehler. Phys. Rev., 100:545, 1955.

${ }^{813}$ J. B. Goodenough. Phys. Rev., 100:564, 1955.

${ }^{814}$ C. H. Chen and S.-W. Cheong. Phys. Rev. Lett., 76:4042, 1996. 
Epitaxial ferroelectric interfacial devices

${ }^{815}$ P. G. Radaelli, D. E. Cox, M. Marezio, and S.-W. Cheong. Phys. Rev. Lett., 55:3015, 1997.

${ }^{816}$ Z. Jirák, S. Krupička, Z. Šimša, M. Dlouhá, and S. Vratislav. J. Magn. Magn. Mater., 53:153, 1985.

${ }^{817}$ M. v. Zimmermann, J. P. Hill, D. Gibbs, M. Blume, D. Casa, B. Keimer, Y. Murakami, Y. Tomioka, and Y. Tokura. Phys. Rev. Lett., 83:4872, 1999.

${ }^{818}$ M. v. Zimmermann, C. S. Nelson, J. P. Hill, D. Gibbs, M. Blume, D. Casa, B. Keimer, Y. Murakami, C.-C. Kao, C. Venkataraman, T. Gog, Y. Tomioka, and Y. Tokura. Phys. Rev. B, 64:195133, 2001.

${ }^{819}$ C. Frontera, J. L. García-Muñoz, M. A. G. Aranda, C. Ritter, A. Llobet, L. Ranno, M. Respaud, J. Vanacken, J. M. Broto, A. E. Carrillo, A. Calleja, and J. García. Appl. Phys. A, 74:S1787, 2002.

${ }^{820}$ B. H. Kim, J. S. Kim, T. H. Park, D. S. Lee, K. H. Kim, B. G. Kim, and Y. W. Park. J. Phys.: Condens. Matter, 19:296205, 2007.

${ }^{821}$ I. El Baggari, B. H. Savitzky, A. S. Admasu, J. Kim, S.-W. Cheong, R. Hovdend, and L. F. Kourkoutis. PNAS, 115:1445, 2018.

${ }^{822}$ R. Comin and A. Damascelli. Annu. Rev. Condens. Matter Phys., 7:369, 2016.

${ }^{823}$ C. Schüßler-Langeheine, J. Schlappa, A. Tanaka, Z. Hu, C. F. Chang, E. Schierle, M. Benomar, H. Ott, E. Weschke, G. Kaindl, O. Friedt, G. A. Sawatzky, H.-J. Lin, C. T. Chen, M. Braden, and L. H. Tjeng. Phys. Rev. Lett., 95:156402, 2005.

${ }^{824}$ J. García and G. Subías. Phys. Rev. B, 68:127101, 2003.

${ }^{825}$ M. v. Zimmermann, S. Grenier, C. S. Nelson, J. P. Hill, D. Gibbs, M. Blume, D. Casa, B. Keimer, Y. Murakami, C.-C. Kao, C. Venkataraman, T. Gog, Y. Tomioka, and Y. Tokura. Phys. Rev. B, 68:127102, 2003.

${ }^{826}$ S. Grenier, J. P. Hill, Doon Gibbs, K. J. Thomas, M. v. Zimmermann, C. S. Nelson, V. Kiryukhin, Y. Tokura, Y. Tomioka, D. Casa, T. Gog, and C. Venkataraman. Phys. Rev. B, 69:134419, 2004.

${ }^{827}$ G. Subías, J. García, M. G. Proietti, and J. Blasco. Phys. Rev. B, 56:8183, 1997.

${ }^{828}$ J. García, M. C. Sánchez, J. Blasco, G. Subías, and M. G. Proietti. J. Phys." Condens. Matter, 13:3243, 2001.

${ }^{829}$ F. Bridges, C. H. Booth, M. Anderson, G. H. Kwei, J. J. Neumeier, J. Snyder, J. Mitchell, J. S. Gardner, and E. Brosha. Phys. Rev. B, 63:214405, 2001. 
Epitaxial ferroelectric interfacial devices

${ }^{830}$ J. Herrero-Martín, J. García, G. Subías, J. Blasco, and M. C. Sánchez. Phys. Rev. B, 70:024408, 2004.

${ }^{831}$ K. J. Thomas, J. P. Hill, S. Grenier, Y-J. Kim, P. Abbamonte, L.Venema, A. Rusydi, Y. Tomioka, Y. Tokura, D. F. McMorrow, G. Sawatzky, and M. van Veenendaal. Phys. Rev. Lett., 92:237204, 2004.

${ }^{832}$ R. J. Goff and J. P. Attfield. Phys. Rev. B, 70:140404(R), 2004.

${ }^{833}$ H. Kawano, R. Kajimoto, H. Yoshizawa, Y. Tomioka, H. Kuwahara, and Y. Tokura. Phys. Rev. Lett., 78:4253, 1997.

${ }^{834}$ J. Herrero-Martín, J. García, G. Subías, J. Blasco, M. C. Sánchez, and S. Stanescu. Phys. Rev. B, 73:224407, 2006.

${ }^{835}$ M. S. Senn, J. P. Wright, and J. P. Attfield. Nature, 481:173, 2012.

${ }^{836}$ M. S. Senn, I. Loa, J. P. Wright, and J. P. Attfield. Phys. Rev. B, 85:125119, 2012.

${ }^{837}$ G. Subías, J. García, J. Blasco, J. Herrero-Martín, M. C. Sánchez, J. Orna, and L. Morellón. J. Synchrotron Rad., 19:159, 2012.

${ }^{838}$ F. Walz. J. Phys.: Condens. Mater, 14:R285, 2002.

${ }^{839}$ J. García and G. Subías. J. Phys.: Condens. Matter, 16:R145, 2004.

${ }^{840}$ C. A. F. Vaz, C. H. Ahn, and V. E. Henrich. Spin-polarisation effects in oxide materials. In A. Hirohata and Y. Otani, editors, Epitaxial ferromagnetic films and spintronic applications, page 145. Research Signpost, Kerala, India, 2009.

${ }^{841}$ E. Pachoud, J. Cumby, G. Perversi, J. P. Wright, and J. P. Attfield. Nature Communications, 11:1671, 2020 .

${ }^{842}$ P. Beaud, S. L. Johnson, E. Vorobeva, U. Staub, R. A. De Souza, C. J. Milne, Q. X. Jia, and G. Ingold. Phys. Rev. Lett., 103:155702, 2009.

${ }^{843}$ T. P. Ma, Y. Yang, Z. Ding, Z. H. Chen, H. B. Zhao, P. Werner, S. S. P. Parkin, and Y. Z. Wu. Phys. Rev. B, 95:014103, 2017.

${ }^{844}$ B. Carter-Stiglitz, B. Moskowitz, P. Solheid, T. S. Berquó, M. Jackson, and A. Kosterov. J. Geophys. Res., 111:B12S05, 2006.

${ }^{845}$ M. Fiebig. J. Phys. D: Appl. Phys., 38:R123, 2005.

${ }^{846}$ N. A. Spaldin and R. Ramesh. MRS Bulletin, 33:1047, 2006.

${ }^{847}$ K. F. Wang, J. M. Liu, and Z. F. Ren. Adv. Phys., 58:321, 2009.

${ }^{848}$ C. A. F. Vaz, J. Hoffman, C. H. Ahn, and R. Ramesh. Adv. Mater., 22:2900, 2010. 
Epitaxial ferroelectric interfacial devices

${ }^{849}$ L. W. Martin, Y.-H. Chu, and R. Ramesh. Materials Science and Engineering R, 68:89, 2010 .

${ }^{850}$ C. A. F. Vaz. J. Phys.: Condens. Matter, 24:333201, 2012.

${ }^{851}$ C. A. F. Vaz and U. Staub. J. Mater. Chem. C, 1:6731, 2013.

${ }^{852}$ S. Dong, J.-M. Liu, S.-W. Cheong, and Z. Ren. Advances in Physics, 64:519, 2015.

${ }^{853}$ C. Lu, W. Hu, Y. Tian, and T. Wu. Appl. Phys. Rev., 2:021304, 2015.

${ }^{854}$ V. Garcia, M. Bibes, and A. Barthélémy. C. R. Physique, 16:168, 2015.

${ }^{855}$ Z. Chu, M. PourhosseiniAsl, and S. Dong. J. Phys. D: Appl. Phys., 51:243001, 2018.

${ }^{856}$ J. F. Scott. J. Mater. Chem., 22:4567, 2012.

${ }^{857}$ S. Fusil, V. Garcia, A. Barthélémy, and M. Bibes. Annu. Rev. Mater. Res., 44:91, 2014.

${ }^{858}$ M. Trassin. J. Phys.: Condens. Matter, 28:033001, 2016.

${ }^{859}$ S. Picozzi, K. Yamauchi, I. A. Sergienko, C. Sen, B. Sanyal, and E. Dagotto. J. Phys.:

Condens. Matter, 20:434208, 2008.

${ }^{860}$ T. Kimura. Annu. Rev. Condens. Mater. Phys., 3:93, 2012.

${ }^{861}$ K. Yamauchi and P. Barone. J. Phys.: Condens. Matter, 26:103201, 2014.

${ }^{862}$ P. S. Halasyamani and K. R. Poeppelmeier. Chem. Mater., 10:2753, 1998.

${ }^{863}$ R. Ramesh and N. A. Spaldin. Nature Mater., 6:21, 2007.

${ }^{864}$ R. Haumont, J. Kreisel, and P. Bouvier. Phase Transitions, 79:1043, 2006.

${ }^{865}$ J. F. Scott. J. Magn. Magn. Mater., 321:1689, 2009.

${ }^{866}$ A. N. Kalinkin and V. M. Skorikov. Russian J. Inorg. Chem., 55:1794, 2010.

${ }^{867}$ F. Matsukura, Y. Tokura, and H. Ohno. Nature Nanotechnology, 10:209, 2015.

${ }^{868}$ C. Song, B. Cui, F. Li, X. Zhou, and F. Pan. Progress in Materials Science, 87:33, 2017.

${ }^{869}$ M. Zhu, T. Nan, B. Peng, Y. Zhang, Z. Zhou, X. Yang, W. Ren, N. X. Sun, and M. Liu. IEEE Transactions on Magnetics, 53:0800116, 2017.

${ }^{870}$ C.-W. Nan, M. I. Bichurin, S. Dong, D. Viehland, and G. Srinivasan. J. Appl. Phys., 103:031101, 2008.

${ }^{871}$ G. Srinivasan. Annu. Rev. Mater. Res., 40:153, 2010.

${ }^{872}$ T. Taniyama. J. Phys.: Condens. Matter, 27:504001, 2015.

${ }^{873}$ A. Chen, Q. Su, H. Han, E. Enriquez, and Q. Jia. Adv. Mater., 31:1803241, 2019.

${ }^{874}$ P. Borisov, A. Hochstrat, X. Chen, W. Kleemann, and C. Binek. Phys. Rev. Lett., 94:117203, 2005.

${ }^{875}$ J. Dho and M. G. Blamire. Appl. Phys. Lett., 87:252504, 2005. 
Epitaxial ferroelectric interfacial devices

${ }^{876}$ L. W. Martin, Y.-H. Chu, M. B. Holcomb, M. Huijben, P. Yu, S.-J. Han, D. Lee, S. X. Wang, and R. Ramesh. Nano Letters, 8:2050, 2008.

${ }^{877}$ Q. Yang, Z. Zhou, N. X. Sun, and M. Liu. Physics Letters A, 381:1213, 2017.

${ }^{878}$ J. Stöhr and H. C. Siegmann. Magnetism. Springer-Verlag, Berlin, 2006.

${ }^{879}$ S. Zhang. Phys. Rev. Lett., 83:640, 1999.

${ }^{880}$ C.-G. Duan, J. P. Velev, R. F. Sabirianov, Z. Zhu, J. Chu, S. S. Jaswal, and E.Y.

Tsymbal. Phys. Rev. Lett., 101:137201, 2008.

${ }^{881}$ J. M. Rondinelli, M. Stengel, and N. A. Spaldin. Nature Nanotechnology, 3:46, 2008.

${ }^{882}$ M. K. Niranjan, C.-G. Duan, S. S. Jaswal, and E. Y. Tsymbal. Appl. Phys. Lett., 96:222504, 2010 .

${ }^{883}$ K. Nakamura, R. Shimabukuro, Y. Fujiwara, T. Akiyama, T. Ito, and A. J. Freeman. Phys. Rev. Lett., 102:187201, 2009.

${ }^{884}$ T. Maruyama, Y. Shiota, T. Nozaki, K. Ohta, N. Toda, M. Mizuguchi, A. A. Tulapurkar, T. Shinjo, M. Shiraishi, S. Mizukami, Y. Ando, and Y. Suzuki. Nature Nanotechnology, $4: 158,2009$

${ }^{885}$ M. Tsujikawa and T. Oda. Phys. Rev. Lett., 102:247203, 2009.

${ }^{886}$ K. Nakamura, T. Akiyama, T. Ito, M. Weinert, and A. J. Freeman. Phys. Rev. B, 81:220409, 2010 .

${ }^{887}$ R. Shimabukuro, K. Nakamura, T. Akiyama, and T. Ito. Physica E, 42:1014, 2010.

${ }^{888}$ S. Haraguchi, M. Tsujikawa, J. Gotou, and T. Oda. J. Phys. D: Appl. Phys., 44:064005, 2011.

${ }^{889}$ B. Heinrich and J. F. Cochran. Adv. Phys., 42:523, 1993.

${ }^{890}$ M. T. Johnson, P. J. H. Bloemen, F. J. A. der Broeder, and J. J. de Vries. Rep. Prog. Phys., 59:1409, 1996.

${ }^{891}$ D. Sander. J. Phys.: Condens. Matter, 16:R603, 2004.

${ }^{892}$ C. A. F. Vaz, J. A. C. Bland, and G. Lauhoff. Rep. Prog. Phys., 71:056501, 2008.

${ }^{893}$ T. Seki, M. Kohda, J. Nitta, and K. Takanashi. Appl. Phys. Lett., 98:212505, 2011.

${ }^{894}$ M. Endo, S. Kanai, S. Ikeda, F. Matsukura, and H. Ohno. Appl. Phys. Lett., 96:212503, 2010.

${ }^{895}$ C. Fowley, N. Decorde, K. Oguz, K. Rode, H. Kurt, and J. M. D. Coey. IEEE Trans. Magn., 46:2116, 2010. 
Epitaxial ferroelectric interfacial devices

${ }^{896}$ C. Fowley, K. Rode, K. Oguz, H. Kurt, and J. M. D. Coey. J. Phys. D: Appl. Phys., 44:305001, 2011.

${ }^{897}$ A. Mardana, S. Ducharme, and S. Adenwalla. Nano Lett., 11:3862, 2011.

${ }^{898}$ J. Heidler, M. Fechner, R. V. Chopdekar, C. Piamonteze, J. Dreiser, C. A. Jenkins, E. Arenholz, S. Rusponi, H. Brune, N. A. Spaldin, and F. Nolting. Phys. Rev. B, 94:014401, 2016.

${ }^{899}$ L. Shu, Z. Li, J. Ma, Y. Gao, L. Gu, Y. Shen, Y. Lin, and C. W. Nan. Appl. Phys. Lett., 100:022405, 2012.

${ }^{900}$ F. Bonell, S. Murakami, Y. Shiota, T. Nozaki, T. Shinjo, and Y. Suzuki. Appl. Phys. Lett., 98:232510, 2011.

${ }^{901}$ Y. Shiota, T. Maruyama, T. Nozaki, T. Shinjo, M. Shiraishi, and Y. Suzuki. Appl. Phys. Express, 2:063001, 2009.

${ }^{902}$ S.-S. Ha, N.-H. Kim, S. Lee, C.-Y. You, Y. Shiota, T. Maruyama, T. Nozaki, and Y. Suzuki. Appl. Phys. Lett., 96:142512, 2010.

${ }^{903}$ K. H. He, J. S. Chen, and Y. P. Feng. Appl. Phys. Lett., 99:072503, 2011.

${ }^{904}$ T. Nozaki, Y. Shiota, M. Shiraishi, T. Shinjo, and Y. Suzuki. Appl. Phys. Lett., 96:022506, 2010.

${ }^{905}$ Y. Shiota, S. Murakami, F. Bonell, T. Nozaki, T. Shinjo, and Y. Suzuki. Applied Physics Express, 4:043005, 2011.

${ }^{906}$ W.-G. Wang, M. Li, S. Hageman, and C. L. Chien. Nature Materials, 11:64, 2012.

${ }^{907}$ H. Meng, R. Sbiaa, M. A. K. Akhtar, R. S. Liu, V. B. Naik, and C. C. Wang. Appl. Phys. Lett., 100:122405, 2012.

${ }^{908}$ T. Zhou, S. H. Leong, Z. M. Yuan, S. B. Hu, C. L. Ong, and B. Liu. Appl. Phys. Lett., 96:012506, 2010 .

${ }^{909}$ C.-G. Duan, S. S. Jaswal, and E.Y. Tsymbal. Phys. Rev. Lett., 97:047201, 2006.

${ }^{910}$ M. Fechner, I. V. Maznichenko, S. Ostanin, A. Ernst, J. Henk, P. Bruno, and I. Mertig. Phys. Rev. B, 78:212406, 2008.

${ }^{911}$ H. L. Meyerheim, F. Klimenta, A. Ernst, K. Mohseni, S. Ostanin, M. Fechner, S. Parihar, I.V. Maznichenko, I. Mertig, and J. Kirschner. Phys. Rev. Lett., 106:087203, 2011.

${ }^{912}$ J.-Q. Dai, Y.-M. Song, and H. Zhang. J. Appl. Phys., 111:114301, 2012.

${ }^{913}$ L. Bocher, A. Gloter, A. Crassous, V. Garcia, Katia March, A Zobelli, S. Valencia, S. Enouz-Vedrenne, X. Moya, N. D. Marthur, C. Deranlot, S. Fusil, K. Bouzehouane, 
Epitaxial ferroelectric interfacial devices

M. Bibes, A. Barthélémy, C. Colliex, and O. Stéphan. Nano Letters, 12:376, 2012.

${ }^{914}$ St. Borek, I. Maznichenko, G. Fischer, W. Hergert, I. Mertig, A. Ernst, S. Ostanin, and

A. Chassé. Phys. Rev. B, 85:134432, 2012.

${ }^{915}$ R. Arras and S. Cherifi-Hertel. ACS Appl. Mater. Interfaces, 11:34399, 2019.

${ }^{916}$ S. Valencia, A. Crassous, L. Bocher, V. Garcia, X. Moya, R. O. Cherifi, C. Deranlot,

K. Bouzehouane, S. Fusil, A. Zobelli, A. Gloter, N. D. Mathur, A. Gaupp, R. Abrudan,

F. Radu, A. Barthélémy, and M. Bibes. Nature Materials, 10:753, 2011.

${ }^{917}$ Y. Liu, J. Tornos, S. G. E. te Velthuis, J. W. Freeland, H. Zhou, P. Steadman, P. Bencok, C. Leon, and J. Santamaria. APL Mater., 4:046105, 2016.

${ }^{918}$ O. Vlašín, R. Jarrier, R. Arras, L. Calmels, B. Warot-Fonrose, C. Marcelot, M. Jamet, P. Ohresser, F. Scheurer, R. Hertel, G. Herranz, and S. Cherifi-Hertel. ACS Appl. Mater. Interfaces, 8:7553, 2016.

${ }^{919}$ P. W. Anderson. Exchange in insulators: Superexchange, direct exchange, and double exchange. In G. T. Rado and H. Suhl, editors, Magnetism, volume I, page 25. Academic Press, New York, 1963.

${ }^{920}$ P. W. Anderson and H. Hasegawa. Phys. Rev., 100:675, 1955.

${ }^{921}$ P.-G. de Gennes. Phys. Rev., 118:141, 1960.

${ }^{922}$ K. Kubo and N. Ohata. J. Phys. Soc. Japan, 33:21, 1972.

${ }^{923}$ E. W. Gorter. Proc. IRE, 43:1945, 1955.

${ }^{924}$ T.R. McGuire and E.L. Boyd. $\mathrm{NiFe}^{3+}$ spinels (Ni ferrites) and $\mathrm{NiFe}^{3+}$ spinels with substitutions. volume 4b of Landolt-Börnstein - Group III: Crystal and Solid State Physics, page 75. Springer-Verlag, Berlin, 1970.

${ }^{925}$ J. B. Goodenough and J.-S. Zhou. Chem. Mater., 10:2980, 1998.

${ }^{926} \mathrm{Yu}$. A. Izyumov and E. Z. Kurmaev. Physics - Uspekhi, 51:23, 2008.

${ }^{927}$ E. Dagotto and Y. Tokura. MRS Bulletin, 33:1037, 2008.

${ }^{928}$ J. B. Goodenough. Progress in Solid State Chemistry, 5:145, 1971.

${ }^{929}$ M. Mochizuki and M. Imada. New J. Phys., 6:154, 2004.

${ }^{930} \mathrm{M}$. Johnsson and P. Lemmens. Crystallography and chemistry of perovskites. In H. Kronmüller and S. Parkin, editors, Handbook of Magnetism and Advanced Magnetic Materials, volume 4, page 1. John Wiley \& Sons, 2007.

${ }^{931}$ M. Johnsson and P. Lemmens. J. Phys.: Condens. Matter, 20:264001, 2008. 
Epitaxial ferroelectric interfacial devices

${ }^{932} \mathrm{I}$. Solovyev and K. Terakura. Orbital degeneracy and magnetism of perovskite manganese oxides. In D. J. Singh and D. A. Papaconstantopoulos, editors, Electronic structure and magnetism of complex materials, page 253. Springer-Verlag, Berlin, 2003.

${ }^{933}$ A.-M. Haghiri-Gosnet and J.-P. Renard. J. Phys. D: Appl. Phys., 36:R127, 2003.

${ }^{934}$ G. H. Jonker and J. H. van Santen. Physica, 16:337, 1950.

${ }^{935}$ B. Roldan Cuenya, M. Doi, W. Keune, S. Hoch, D. Reuter, A. Wieck T. Schmitte, and H. Zabel. Appl. Phys. Lett., 82:1072, 2003.

${ }^{936}$ R. P. Borges, W. Guichard, J. G. Lunney, J. M. D. Coey, and F. Ott. J. Appl. Phys., 89:3868, 2001.

${ }^{937}$ M. Izumi, Y. Ogimoto, T. Manako, M. Kawasaki, and Y. Tokura. J. Phys. Soc. Japan, $71: 2621,2002$.

${ }^{938}$ J. J. Kavich, M. P. Warusawithana, J. W. Freeland, P. Ryan, X. Zhai, R. H. Kodama, and J. N. Eckstein. Phys. Rev. B, 76:014410, 2007.

${ }^{939}$ J. W. Freeland, J. J. Kavich, K. E. Gray, L Ozyuzer, H Zheng, J. F. Mitchell, M. P. Warusawithana, P. Ryan, X. Zhai, R. H. Kodama, and J. N. Eckstein. J. Phys.: Condens. Matter, 19:315210, 2007.

${ }^{940}$ M. Huijben, L. W. Martin, Y.-H. Chu, M. B. Holcomb, P. Yu, G. Rijnders, D. H. A. Blank, and R. Ramesh. Phys. Rev. B, 78:094413, 2008.

${ }^{941}$ L. F. Kourkoutis, J. H. Song, H. Y. Hwang, and D. A. Muller. PNAS, 107:11682, 2010. ${ }^{942}$ J.-S. Lee, D. A. Arena, P. Yu, C. S. Nelson, R. Fan, C. J. Kinane, S. Langridge, M. D. Rossell, R. Ramesh, and C.-C. Kao. Phys. Rev. Lett., 105:257204, 2010.

${ }^{943}$ H. Boschker, J. Kautz, E. P. Houwman, W. Siemons, D. H. A. Blank, M. Huijben, G. Koster, A. Vailionis, and G. Rijnders. Phys. Rev. Lett., 109:157207, 2012.

${ }^{944}$ L. Chen, Z. Wang, G. Wang, H. Guo, M. Saghayezhian, Z. Liao, Y. Zhu, E. W. Plummer, and J. Zhang. Phys. Rev. Materials, 3:044407, 2019.

${ }^{945}$ C. A. F. Vaz, J. Hoffman, Y. Segal, M. S. J. Marshall, J. W. Reiner, Z. Zhang, R. D. Grober, F. J. Walker, and C. H. Ahn. J. Appl. Phys., 109:07D905, 2011.

${ }^{946}$ T. Kanki, H. Tanaka, and T. Kawai. Appl. Phys. Lett., 89:242506, 2006.

${ }^{947}$ Z. Fang, I. V. Solovyev, and K. Terakura. Phys. Rev. Lett., 84:3169, 2000.

${ }^{948}$ J. D. Burton and E. Y. Tsymbal. Phys. Rev. B, 80:174406, 2009.

${ }^{949}$ S. Dong, X. Zhang, R. Yu, J.-M. Liu, and E. Dagotto. Phys. Rev. B, 84:155117, 2011. 
Epitaxial ferroelectric interfacial devices

${ }^{950}$ N. C. Bristowe, M. Stegel, P. B. Littlewood, J. M. Pruneda, and E. Artacho. Phys. Rev. $B, 85: 024106,2012$.

${ }^{951}$ H. Chen and S. Ismael-Beigi. Phys. Rev. B, 86:024433, 2012.

${ }^{952}$ S. Dong and E. Dagotto. Phys. Rev. B, 88:140404(R), 2013.

${ }^{953}$ M. Hammouri, E. Fohtung, and I. Vasiliev. J. Phys.: Condens. Matter, 28:396004, 2016.

${ }^{954}$ C. A. F. Vaz, Y. Segal, J. Hoffman, R. D. Grober, F. J. Walker, and C. H. Ahn. Appl. Phys. Lett., 97:042506, 2010.

${ }^{955}$ C. A. F. Vaz, J. Hoffman, Y. Segal, F. J. Walker, and C. H. Ahn. Proc. of SPIE, 7760:776013, 2010.

${ }^{956}$ H. Lu, T. A. George, Y. Wang, I. Ketsman, J. D. Burton, C.-W. Bark, S. Ryu, D. J. Kim, J. Wang, C. Binek, P. A. Dowben, A. Sokolov, C.-B. Eom, E. Y. Tsymbal, and A. Gruverman. Appl. Phys. Lett., 100:232904, 2012.

${ }^{957}$ A. M. Kane, I.-T. Chiu, N. J. Ahlm, R. V. Chopdekar, A. T. N'Diaye, E. Arenholz, A. Mehta, V. Lauter, and Y. Takamura. ACS Appl. Mater. Interfaces, 12:45437, 2020. ${ }^{958}$ P. M. Leufke, R. Kruk, R. A. Brand, and H. Hahn. Phys. Rev. B, 87:094416, 2013.

${ }^{959}$ D. Preziosi, I. Fina, E. Pippel, D. Hesse, X. Marti, F. Bern, M. Ziese, and M. Alexe. Phys. Rev. B, 90:125155, 2014.

${ }^{960}$ T. L. Meyer, A. Herklotz, V. Lauter, J. W. Freeland, J. Nichols, E.-J. Guo, S. Lee, T. Z. Ward, N. Balke, S. V. Kalinin, M. R. Fitzsimmons, and H. N. Lee. Phys. Rev. B, 94:174432, 2016.

${ }^{961}$ J. Ma, Y. Tian, M. Chen, J. Wang, J. Ma, J. Zhang, and C.-W. Nan. J. Appl. Phys., 126:075301, 2019.

${ }^{962}$ D. Yi, P. Yu, Y.-C. Chen, H.-H. Lee, Q. He, Y.-H. Chu, and R. Ramesh. Adv. Mater., $31: 1806335,2019$.

${ }^{963}$ B. Paudel, I. Vasiliev, M. Hammouri, D. Karpov, A. Chen, V. Lauter, and E. Fohtung. RSC Adv., 9:13033, 2019.

${ }^{964}$ C. Liu, Y. Liu, B. Zhang, C.-J. Sun, D. Lan, P. Chen, X. Wu, P. Yang, X. Yu, T. Charlton, M. R. Fitzsimmons, J. Ding, J. Chen, and G. M. Chow. ACS Appl. Mater. Interfaces, 13:30137, 2021.

${ }^{965}$ X. Ma, A. Kumar, S. Dussan, H. Zhai, F. Fang, H. B. Zhao, J. F. Scott, R. S. Katiyar, and G. Lüpke. Appl. Phys. Lett., 104:132905, 2014.

${ }^{966}$ J. F. Ankner and G. P. Felcher. J. Magn. Magn. Mater., 200:741, 1999. 
Epitaxial ferroelectric interfacial devices

${ }^{967}$ C. F. Majkrzak. Physica B, 221:342, 1996.

${ }^{968}$ C. Fermon, F. Ott, and A. Menelle. Neutron reflectometry. In J. Daillant and A. Gibaud, editors, X-Ray and Neutron Reflectivity: Principles and Applications, page 163. Springer, Berlin, 1999.

${ }^{969}$ J. A. C. Bland and C. A. F. Vaz. Polarised neutron reflection studies of thin magnetic films. In J. A. C. Bland and B. Heinrich, editors, Ultrathin Magnetic Structures III, page 233. Springer-Verlag, Berlin, 2005.

${ }^{970}$ H. Zabel, K. Theis-Bröhl, and B. P. Toperverg. Polarized neutron reflectivity and scattering of magnetic nanostructures and spintronic materials. In H. Kronmüller and S. Parkin, editors, Handbook of Magnetism and Advanced Magnetic Materials, page 1237. John Wiley \& Sons, Hoboken, 2007.

${ }^{971}$ M. Varela, M. P. Oxley, W. Luo, J. Tao, M. Watanabe, A. R. Lupini, S. T. Pantelides, and S. J. Pennycook. Phys. Rev. B, 79:085117, 2009.

${ }^{972}$ W. Huang, Y. Yin, and X. Li. Appl. Phys. Rev., 5:041110, 2018.

${ }^{973}$ A. Y. Borisevich, H. J. Chang, M. Huijben, M. P. Oxley, S. Okamoto, M. K. Niranjan, J. D. Burton, E. Y. Tsymbal, Y. H. Chu, P. Yu, R. Ramesh, S. V. Kalinin, and S. J. Pennycook. Phys. Rev. Lett., 105:087204, 2010.

${ }^{974}$ S. Zhang, X. Guo, Y. Tang, D. Ma, Y. Zhu, Y. Wang, S. Li, M. Han, D. Chen, J. Ma, B. Wu, and X. Ma. ACS Nano, 12:3681, 2018.

${ }^{975}$ F. Y. Bruno, S. Boyn, S. Fusil, S. Girod, C. Carrétéro, M. Marinova, A. Gloter, S. Xavier, C. Deranlot, M. Bibes, A. Barthélémy, and V. Garcia. Adv. Electron. Mater., 2:1500245, 2016.

${ }^{976} \mathrm{R}$. A. Lefever. $\mathrm{Fe}^{3+}$ spinels. volume $12 \mathrm{~b}$ of Landolt-Börnstein - Group III: Crystal and Solid State Physics, page 55. Springer-Verlag, Berlin, 1980.

${ }^{977}$ H. Ji, Y. G. Wang, and Y. Li. J. Magn. Magn. Mater., 442:242, 2017.

${ }^{978}$ H. Ji, Y. G. Wang, and Y. Li. J. Phys.: Condens. Matter, 30:11LT01, 2018.

${ }^{979}$ H. Wu, S. D. Zhou, Y. Li, Y. G. Wang, and F. M. Pan. Appl. Phys. Lett., 113:102404, 2018.

${ }^{980}$ Y. L. Lu, H. Wu, L. Zhu, Y. Li, Y. G. Wang, and F. M. Pan. Appl. Phys. Lett., 114:052401, 2019.

${ }^{981}$ C. Moutafis, S. Komineas, C. A. F. Vaz, J. A. C. Bland, T. Shima, T. Seki, and K. Takanashi. Phys. Rev. B, 76:104426, 2007. 
Epitaxial ferroelectric interfacial devices

${ }^{982}$ F. Büttner, C. Moutafis, M. Schneider, B. Krüger, C. M. Günther, J. Geilhufe, C. v. Korff, Schmising, J. Mohanty, B. Pfau, S. Schaffert, A. Bisig, M. Foerster, T. Schulz, C. A. F. Vaz, J. H. Franken, H. J. M. Swagten, M. Kläui, and S. Eisebitt. Nature Physics, 11:225, 2015.

${ }^{983}$ C. H. Marrows. Physics, 8:40, 2015.

${ }^{984}$ S. Mühlbauer, B. Binz, F. Jonietz, C. Pfleiderer, A. Rosch, A. Neubauer, R. Georgii, and P. Böni. Science, 323:915, 2009.

${ }^{985}$ X. Z. Yu, Y. Onose, N. Kanazawa, J. H. Park, J. H. Han, Y. Matsui, N. Nagaosa, and Y. Tokura. Nature, 465:901, 2010.

${ }^{986}$ S. Heinze, K. von Bergmann, M. Menzel, J. Brede, A. Kubetzka, R. Wiesendanger, G. Bihlmayer, and S. Blügel. Nature Physics, 7:713, 2011.

${ }^{987}$ J. Sampaio, V. Cros, S. Rohart, A. Thiaville, and A. Fert. Nature Nanotechnology, 8:839, 2013.

${ }^{988}$ A. Hrabec, N. A. Porter, A. Wells, M. J. Benitez, G. Burnell, S. McVitie, D. McGrouther, T. A. Moore, and C. H. Marrows. Phys. Rev. B, 90:020402, 2014.

${ }^{989}$ C. Moreau-Luchaire, C. Moutafis, N. Reyren, J. Sampaio, C. A. F. Vaz, N. Van Horne, K. Bouzehouane, K. Garcia, C. Deranlot, P. Warnicke, P. Wohlhueter, J.-M. George, M. Weigand, J. Raabe, V. Cros, and A. Fert. Nature Nanotechnology, 11:444, 2016.

${ }^{990}$ W. Jiang, G. Chen, K. Liu, J. Zang, S. G. E. te Velthuis, and A. Hoffmann. Physics Reports, 704:1, 2017.

${ }^{991}$ A. Soumyanarayanan, M. Raju, A. L. G. Oyarce, A. K. C. Tan, M. Y. Im, A. P. Petrovic, P. Ho, K. H. Khoo, M. Tran, C. K. Gan, F. Ernult, and C. Panagopoulos. Nature Materials, 16:898, 2017.

${ }^{992}$ K. Y. Meng, A. S. Ahmed, M. Bacani, A. O. Mandru, X. Zhao, N. Bagues, B. D. Esser, J. Flores, D. W. McComb, H. J. Hug, and F. Yang. Nano Lett., 19:3169, 2019.

${ }^{993}$ J. Matsuno, N. Ogawa, K. Yasuda, F. Kagawa, W. Koshibae, N. Nagaosa, Y. Tokura, and M. Kawasaki. Sci. Adv., 2:e1600304, 2016.

${ }^{994}$ Q. Qin, L. Liu, W. Lin, X. Shu, Q. Xie, Z. Lim, C. Li, S. He, G. M. Chow, and J. Chen. Adv. Mater., 31:e1807008, 2019.

${ }^{995}$ M. Ziese, L. Jin, and I. Lindfors-Vrejoiu. Journal of Physics: Materials, 2:34008, 2019.

${ }^{996}$ L. F. Mattheiss. Phys. Rev. B, 13:2433, 1976. 
Epitaxial ferroelectric interfacial devices

${ }^{997}$ L. Wang, Q. Feng, Y. Kim, R. Kim, K. H. Lee, S. D. Pollard, Y. J. Shin, H. Zhou, W. Peng, D. Lee, W. Meng, H. Yang, J. H. Han, M. Kim, Q. Lu, and T. W. Noh. Nat. Mater., 17:1087, 2018.

${ }^{998}$ Y. Gu, C. Song, Q. Zhang, F. Li, H. Tan, K. Xu, J. Li, M. S. Saleem, M. U. Fayaz, J. Peng, F. Hu, L. Gu, W. Liu, Z. Zhang, and F. Pan. ACS Appl Mater Interfaces, 12(5):6707, 2020.

${ }^{999}$ T. Dietl, H. Ohno, F. Matsukura, J. Cibert, and D. Ferrand. Science, 287:1019, 2000.

${ }^{1000}$ T. Dietl, H. Ohno, and F. Matsukura. Phys. Rev. B, 63:195205, 2001.

${ }^{1001}$ T. Dietl. Semicond. Sci. Technol., 17:377, 2002.

${ }^{1002}$ P. Mahadevan, A. Zunger, and D. D. Sarma. Phys. Rev. Lett., 93:177201, 2004.

${ }^{1003}$ T. Jungwirth, J. Sinova, J. Mašek, J. Kučera, and A. H. MacDonald. Rev. Mod. Phys., $78: 809,2006$.

${ }^{1004}$ T. Dietl. Nature Materials, 9:965, 2010.

${ }^{1005}$ A. Zunger, S. Lany, and H. Raebiger. Physics, 3:53, 2010.

${ }^{1006}$ K. Sato, L. Bergqvist, J. Kudrnovský, P. H. Dederichs, O. Eriksson, I. Turek, B. Sanyal, G. Bouzerar, H. Katayama-Yoshida, V. A. Dinh, T. Fukushima, H. Kizaki, and R. Zeller. Rev. Mod. Phys., 82:1633, 2010.

${ }^{1007}$ H. Ohno, D. Chiba, F. Matsukura, T. Omiya, E. Abe, T. Dietl, Y. Ohno, and K. Ohtani. Nature, 408:944, 2000.

${ }^{1008}$ D. Chiba, M. Yamanouchi, F. Matsukura, and H. Ohno. Science, 301:943, 2003.

${ }^{1009}$ Y. D. Park, A. T. Hanbicki, S. C. Erwin, C. S. Hellberg, J. M. Sullivan, J. E. Mattson, T. F. Ambrose, A. Wilson, G. Spanos, and B. T. Jonker. Science, 295:651, 2002.

${ }^{1010}$ J. Chen, K. L. Wang, and K. Galatsis. Appl. Phys. Lett., 90:012501, 2007.

${ }^{1011}$ F. Xiu, Y. Wang, J. Kim, A. Hong, J. Tang, A. P. Jacob, J. Zou, and K. L. Wang. Nature Materials, 9:337, 2010.

${ }^{1012}$ M. K. Kneip, D. R. Yakovlev, M. Bayer, T. Slobodskyy, G. Schmidt, and L. W. Molenkamp. Appl. Phys. Lett., 88:212105, 2006.

${ }^{1013}$ A. M. Nazmul, S. Kobayashi, S. Sugahara, and M. Tanaka. Physica E, 21:937, 2004.

${ }^{1014}$ I. Stolichnov, S. W. E. Riester, H. J. Trodahl, N. Setter, A. W. Rushforth, K. W. Edmonds, R. P. Campion, C. T. Foxon, B. L. Gallagher, and T. Jungwirth. Nature Mater., 7:464, 2008. 
Epitaxial ferroelectric interfacial devices

${ }^{1015}$ D. Chiba, M. Sawicki, Y. Nishitani, Y. Nakatani, F. Matsukura, and H. Ohno. Nature, 455:515, 2008.

${ }^{1016}$ M. H. S. Owen, J. Wunderlich, V. Novák, K. Olejník, J. Zemen, K. Výborný, S. Ogawa, A. C. Irvine, A. J. Ferguson, H. Sirringhaus, and T. Jungwirth. New J. Phys., 11:023008, 2009.

${ }^{1017}$ S. W. E. Riester, I. Stolichnov, H. J. Trodahl, N. Setter, A. W. Rushforth, K. W. Edmonds, R. P. Campion, C. T. Foxon, B. L. Gallagher, and T. Jungwirth. Appl. Phys. Lett., 94:063504, 2009.

${ }^{1018}$ M. Endo, D. Chiba, H. Shimotani, F. Matsukura, Y. Iwasa, and H. Ohno. Appl. Phys. Lett., 96:022515, 2010.

${ }^{1019}$ I. Stolichnov, S. W. E. Riester, E. Mikheev, N. Setter, A. W. Rushforth, K. W. Edmonds, R. P. Campion, C. T. Foxon, B. L. Gallagher, T. Jungwirth, and H. J. Trodahl. Phys. Rev. B, 83:115203, 2011.

${ }^{1020}$ T. Zhao, S. R. Shinde, S. B. Ogale, H. Zheng, T. Venkatesan, R. Ramesh, and S. Das Sarma. Phys. Rev. Lett., 94:126601, 2005.

${ }^{1021}$ A. Kaminski and S. Das Sarma. Phys. Rev. Lett., 88:247202, 2002.

${ }^{1022}$ S. Das Sarma, E. H. Hwang, and A. Kaminski. Phys. Rev. B, 67:155201, 2003.

${ }^{1023}$ A. Kaminski and S. Das Sarma. Phys. Rev. B, 68:235210, 2003.

${ }^{1024}$ J. M. D. Coey, M. Venkatesan, and C. B. Fitzgerald. Nature Materials, 4:173, 2005.

${ }^{1025}$ L. Esaki, R.B Laibowitz, and P.J. Stiles. IBM. Tech. Discl. Bull., 13:2161, 1971.

${ }^{1026}$ I. Giaever. Physical Review Letters, 5:147, 1960.

${ }^{1027}$ I. Giaever. Physical Review Letters, 5:470, 1960.

${ }^{1028}$ J. Moodera, L. Kinder, T. Wong, and R. Meservey. Phys. Rev. Lett., 74:3273, 1995.

${ }^{1029}$ C. Lichtensteiger, J.-M. Triscone, J. Junquera, and P. Ghosez. Physical Review Letters, 94:047603, 2005.

${ }^{1030}$ D. A. Tenne, A. Bruchhausen, N. D. Lanzillotti-Kimura, A. Fainstein, R. S. Katiyar, A. Cantarero, A. Soukiassian, V. Vaithyanathan, J. H. Haeni, W. Tian, D. G. Schlom, K. J. Choi, D. M. Kim, C. B. Eom, H. P. Sun, X. Q. Pan, Y. L. Li, L. Q. Chen, Q. X. Jia, S. M. Nakhmanson, K. M. Rabe, and X. X. Xi. Science, 313:1614, 2006.

${ }^{1031}$ M. Ye. Zhuravlev, R. F. Sabirianov, S. S. Jaswal, and E. Y. Tsymbal. Physical Review Letters, 94:246802, 2005.

${ }^{1032}$ V. Garcia and M. Bibes. Nat. Commun., 5:4289, 2014. 
Epitaxial ferroelectric interfacial devices

1033Z. Wen and D. Wu. Adv. Mater., n/a:1904123, 2019.

${ }^{1034}$ S. Boyn, V. Garcia, S. Fusil, C. Carrétéro, K. Garcia, S. Xavier, S. Collin, C. Deranlot, M. Bibes, and A. Barthélémy. APL Materials, 3:061101, 2015.

${ }^{1035}$ Z. Wen, D. Wu, A. Li, and N. Ming. Nature Materials, 12:617, 2013.

${ }^{1036}$ S. Boyn, A.M. Douglas, C. Blouzon, P. Turner, A. Barthélémy, M. Bibes, S. Fusil, J.M.

Gregg, and V. Garcia. Appl. Phys. Lett., 109:232902, 2016.

${ }^{1037}$ Z. Li, X. Guo, H.-B. Lu, Z. Zhang, D. Song, S. Cheng, M. Bosman, J. Zhu, Z. Dong, and W. Zhu. Advanced Materials, 26:7185, 2014.

${ }^{1038}$ F. Ambriz-Vargas, G. Kolhatkar, R. Thomas, R. Nouar, A. Sarkissian, C. Gomez-Yáñez, M.A. Gauthier, and A. Ruediger. Applied Physics Letters, 110:093106, 2017.

${ }^{1039}$ F. Ambriz-Vargas, G. Kolhatkar, M. Broyer, A. Hadj-Youssef, R. Nouar, A. Sarkissian,

R. Thomas, C. Yáñez, M.A. Gauthier, and A. Ruediger. ACS Applied Materials $\mathcal{E}$ Interfaces, 9:13262, 2017.

${ }^{1040}$ Y. Wei, S. Matzen, T. Maroutian, G. Agnus, M. Salverda, P. Nukala, J. Ye Q. Chen,

P. Lecoeur, and B. Noheda. Phys. Rev. Applied, 12:031001, 2019.

${ }^{1041}$ A. Chanthbouala, V. Garcia, R. O. Cherifi, K. Bouzehouane, S. Fusil, X. Moya, S. Xavier,

H. Yamada, C. Deranlot, N. D. Mathur, M. Bibes, A. Barthélémy, and J. Grollier. Nature Mater., 11:860, 2012.

${ }^{1042}$ L. Chua. IEEE Transactions on Circuit Theory, 18:507, 1971.

${ }^{1043}$ L. Chua. Applied Physics A, 102:765, 2011.

${ }^{1044}$ M. Julliere. Phys. Lett. A, 54:225, 1975.

${ }^{1045}$ V. Garcia, M. Bibes, L. Bocher, S. Valencia, F. Kronast, A. Crassous, X. Moya, S. Enouz-

Vedrenne, A. Gloter, D. Imhoff, C. Deranlot, N. D. Mathur, S. Fusil, K. Bouzehouane, and A. Barthélémy. Science, 327:1106, 2010.

${ }^{1046}$ D. Pantel, S. Goetze, D. Hesse, and M. Alexe. Nature Materials, 11:289, 2012.

${ }^{1047}$ Y. W. Yin, J. D. Burton, Y.-M. Kim, A. Y. Borisevich, S. J. Pennycook, S. M. Yang,

T. W. Noh, A. Gruverman, X. G. Li, E. Y. Tsymbal, and Q. Li. Nature Materials, 12:397, 2013.

${ }^{1048}$ Y. A. Bychkov and E. I. Rashba. JETP Lett., 39:78, 1984.

${ }^{1049}$ A. Manchon, H. C. Koo, J. Nitta, S. M. Frolov, and R. A. Duine. Nature Materials, 14:871, 2015.

${ }^{1050}$ J. Varignon, L. Vila, A. Barthélémy, and M. Bibes. Nature Phys., 14:322, 2018. 
Epitaxial ferroelectric interfacial devices

${ }^{1051}$ V. M. M. Edelstein. Solid State Communications, 73:233, 1990.

1052 J. C. Rojas-Sánchez, L. Vila, G. Desfonds, S. Gambarelli, J. P. Attané, J. M. De Teresa,

C. Magén, and A. Fert. Nature Commun., 4:2944, 2013.

${ }^{1053}$ K. Ishizaka, M. S. Bahramy, H. Murakawa, M. Sakano, T. Shimojima, T. Sonobe, K. Koizumi, S. Shin, H. Miyahara, A. Kimura, K. Miyamoto, T. Okuda, H. Namatame, M. Taniguchi, R. Arita, N. Nagaosa, K. Kobayashi, Y. Murakami, R. Kumaiv Y. Kaneko, Y. Onose, and Y. Tokura. Nature Materials, 10:521, 2011.

${ }^{1054}$ S. Picozzi. Frontiers in Physics, 2:10, 2014.

${ }^{1055}$ D. Di Sante, R. Bertacco P. Barone and, and S. Picozzi. Advanced Materials, 25:509, 2013.

${ }^{1056}$ A. V. Kolobov, D.J. Kim, A. Giussani, P. Fons, J. Tominaga, R. Calarco, and A. Gruverman. APL Materials, 2:066101, 2014.

${ }^{1057}$ S. Varotto, L. Nessi, S. Cecchi, J. Sławińska, P. Noël, S. Petrò, F. Fagiani, A. Novati, M. Cantoni, D. Petti, E. Albisetti, M. Costa, R. Calarco, M. B. Nardelli, M. Bibes, S. Picozzi, J.-P. Attané, L. Vila, R. Bertacco, and C. Rinaldi. arXiv:2103.07646 [condmat.mtrl-sci].

${ }^{1058}$ C. Rinaldi, S. Varotto, M. Asa, J. Sławińska, J. Fujii, G. Vinai, S. Cecchi, D. Di Sante, R. Calarco, I. Vobornik, G. Panaccione, S. Picozzi, and R. Bertacco. Nano Letters, 18:2751, 2018 .

${ }^{1059}$ J. Krempaský, S. Muff, J. Minár, N. Pilet, M. Fanciulli, A. P. Weber, E. B. Guedes, M. Caputo, E. Müller, V. V. Volobuev, M. Gmitra, C. A. F. Vaz, V. Scagnoli, G. Springholz, and J. H. Dil. Phys. Rev. X, 8:021067, 2018.

${ }^{1060}$ L. G. D. da Silveira, P. Barone, and S. Picozzi. Physical Review B, 93:245159, 2016.

${ }^{1061}$ L. L. Tao and J. Wang. Journal of Applied Physics, 120:234101, 2016.

${ }^{1062}$ J. Varignon, J. Santamaria, and M. Bibes. ArXiv, 1808.03123:n/a, 2018.

${ }^{1063}$ H. Djani, A. C. Garcia-Castro, W.-Y. Tong, P. Barone, E. Bousquet, S. Picozzi, and P. Ghosez. Npj Quantum Mater., 4:51, 2019.

${ }^{1064}$ P. Noël, F. Trier, L. M. V. Arche, J. Bréhin, D. C. Vaz, V. Garcia, S. Fusil, A. Barthélémy, L. Vila, M. Bibes, and J.-P. Attané. Nature, 580:483, 2020.

${ }^{1065}$ H. Mirhosseini, I. V. V. Maznichenko, S. Abdelouahed, S. Ostanin, A. Ernst, I. Mertig, and J. Henk. Physical Review B, 81:2, 2010. 
Epitaxial ferroelectric interfacial devices

${ }^{1066}$ Z. C. Zhong, L. Si, Q. F. Zhang, W. G. Yin, S. Yunoki, and K. Held. Advanced Materials Interfaces, 2:5, 2015.

${ }^{1067}$ Y. Song, D. Zhang, B. Xu, K. Chang, and C.W. Nan. ArXiv, 1909.03727:n/a, 2019.

${ }^{1068}$ E. Lesne, Y. Fu, S. Oyarzun, J. C. Rojas-Sánchez, D. C. Vaz, H. Naganuma, G. Sicoli, J.-P. Attané, M. Jamet, E. Jacquet, J.-M. George, A. Barthélémy, H. Jaffrès, A. Fert, M. Bibes, and L. Vila. Nature Materials, 15:1261, 2016.

${ }^{1069}$ D. C. Vaz, P. Noël, A. Johansson, B. Göbel, F. Y. Bruno, G. Singh, S. McKeown-Walker, F. Trier, L. M. Vicente-Arche, A. Sander, S. Valencia, P. Bruneel, M. Vivek, M. Gabay, N. Bergeal, F. Baumberger, H. Okuno, A. Barthélémy, A. Fert, L. Vila, I. Mertig, J.-P. Attané, and M. Bibes. Nature Materials, 18:1187, 2019.

${ }^{1070}$ D. C. Vaz, F. Trier, A. Dyrdał, A. Johansson, K. Garcia, A. Barthélémy, I. Mertig, J. Barnaś, A. Fert, and M. Bibes. Phys. Rev. Materials, 4:071001(R), 2020.

${ }^{1071}$ F. Trier, D. C. Vaz, P. Bruneel, P. Noël, A. Fert, L. Vila, J.-P. Attané, A. Barthélémy, M. Gabay, H. Jaffrès, and M. Bibes. Nano Lett., 20:395, 2020.

${ }^{1072}$ J. Hemberger, P. Lunkenheimer, R. Viana, R. Bohmer, and A. Loidl. Phys. Rev. B, $52: 13159,1995$ 

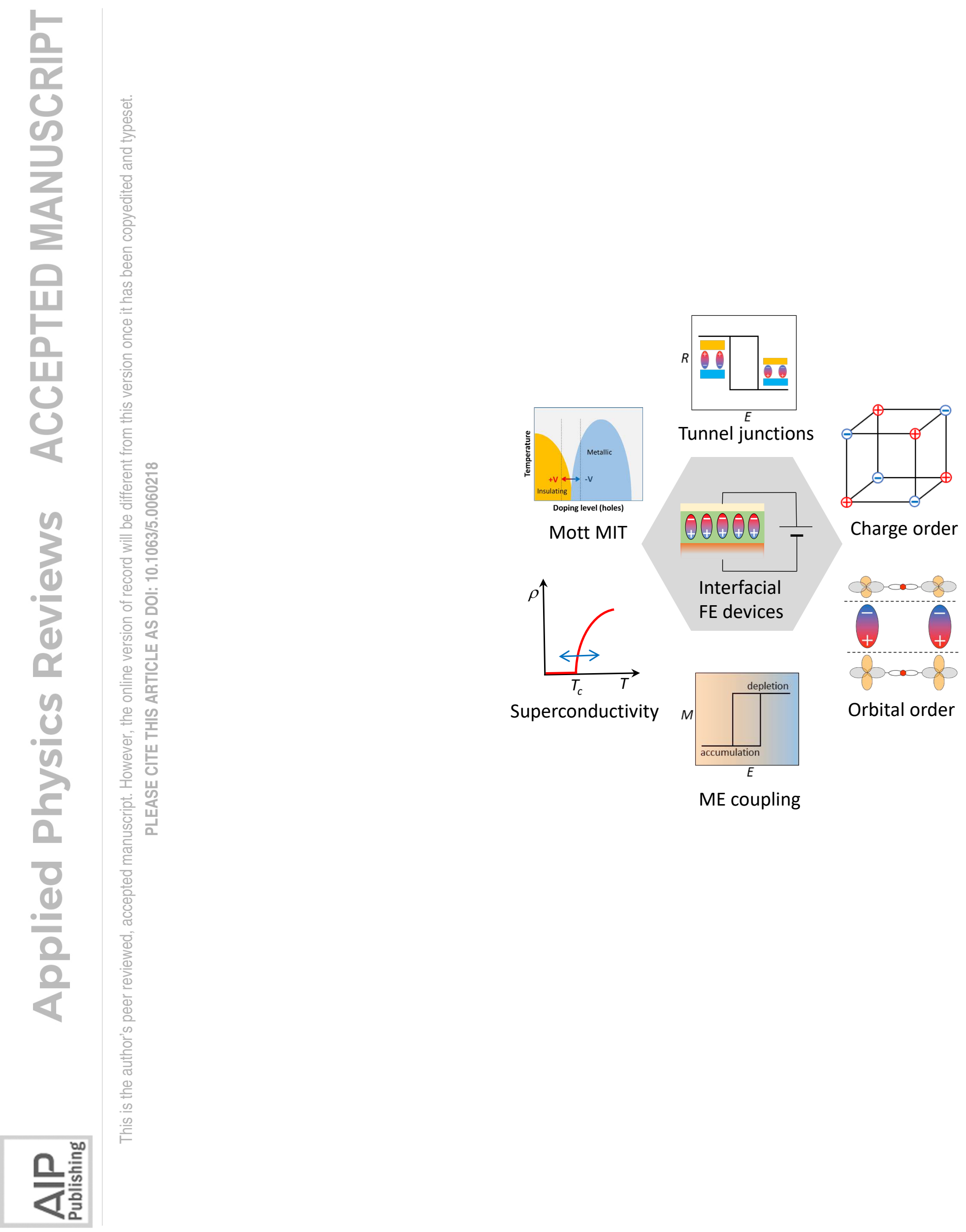
a)

Gate

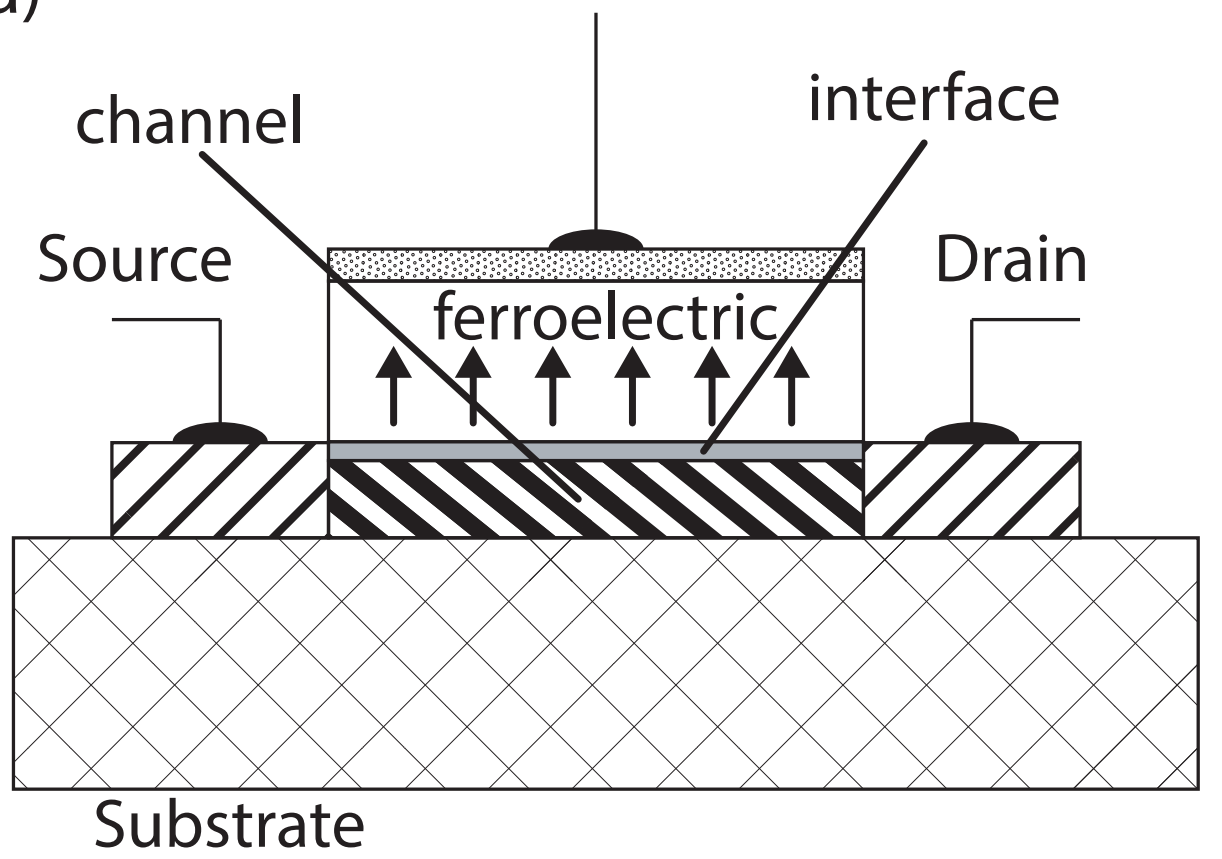

b)

Current/spin source

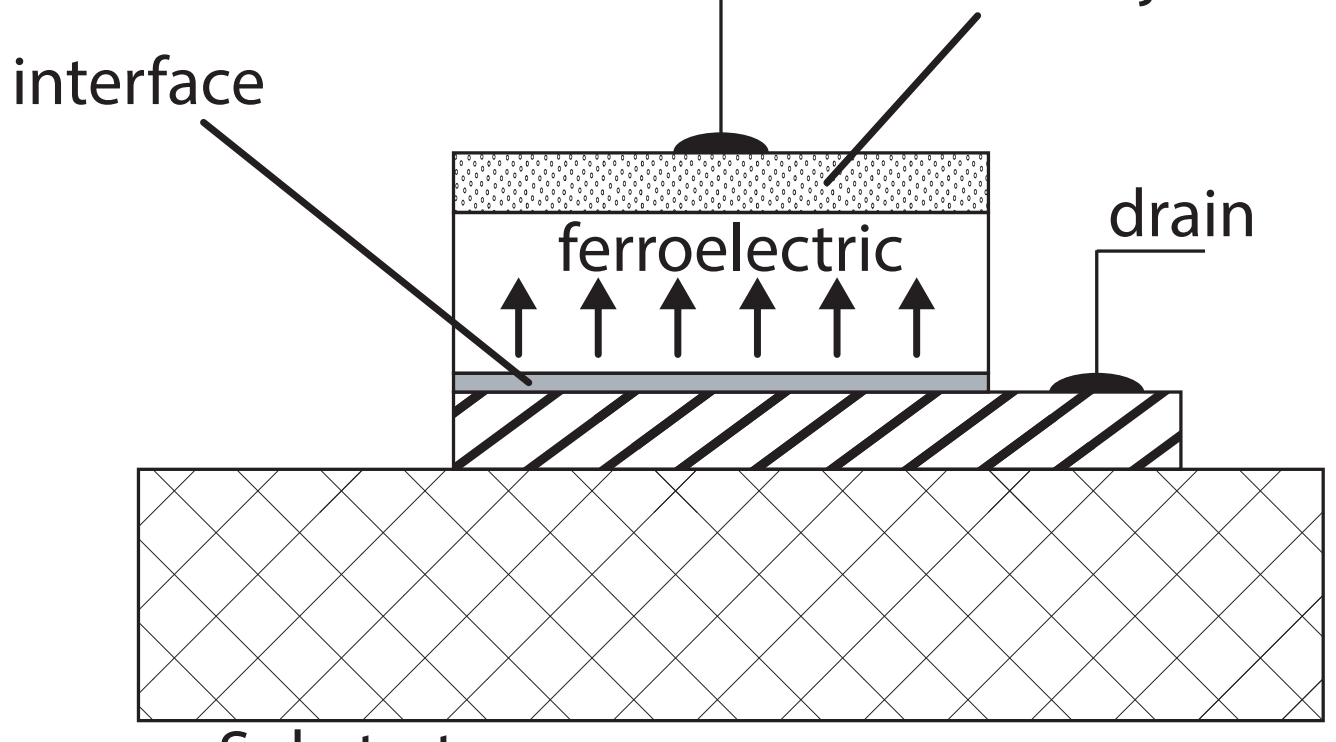

Substrate 


\section{ature of the $\mathrm{BaTiO}_{3}$ layer was $900{ }^{\circ} \mathrm{C}$, which was optimized}

(a)
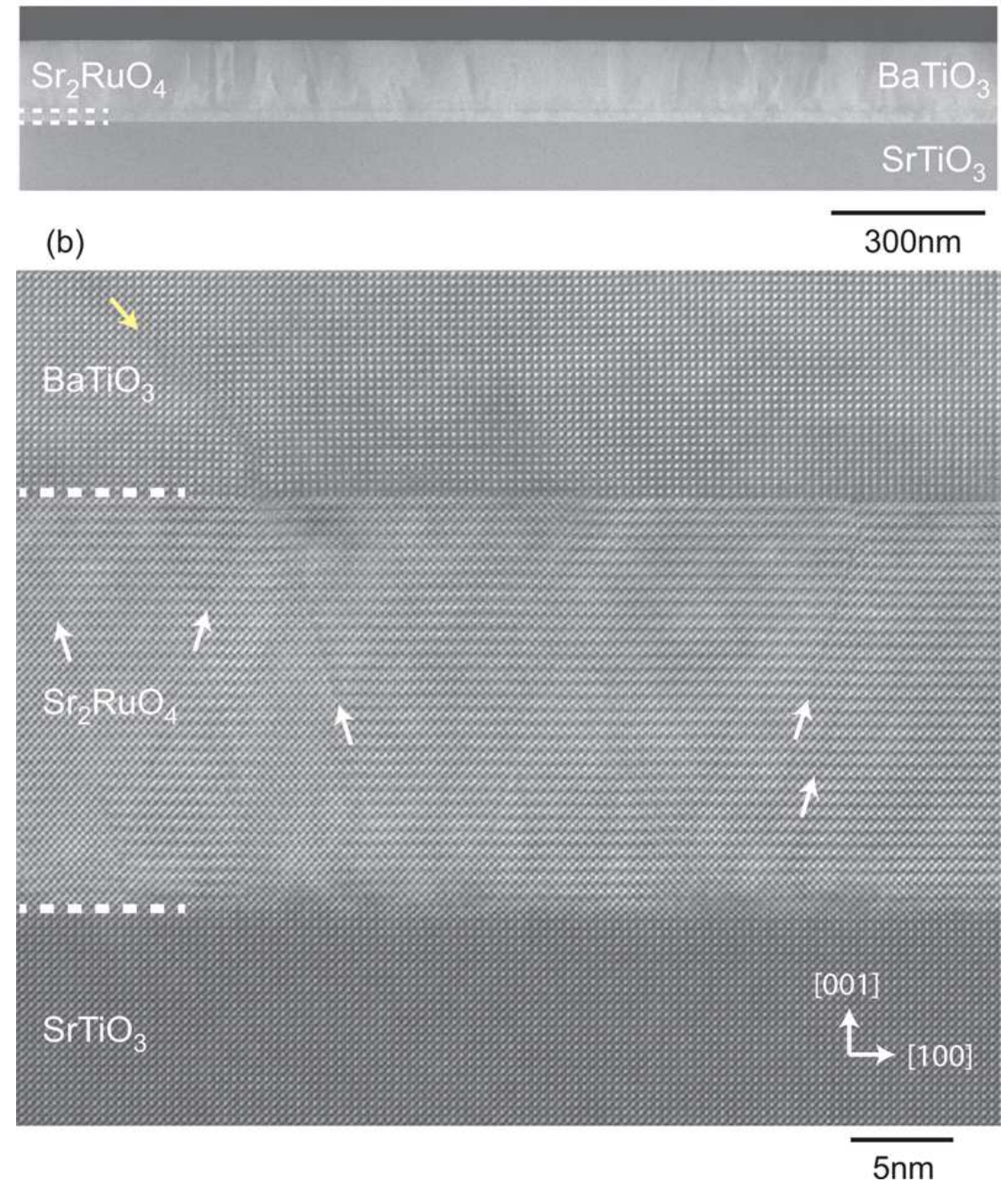

Figure 5. Cross-sectional HAADF-STEM images of $\mathrm{BaTiO}_{3}$ fi grown on $\mathrm{Sr}_{2} \mathrm{RuO}_{4}(001)$ electrodes at $900{ }^{\circ} \mathrm{C}$. (a) Low- and (b) hig magnification image. Dotted lines denote the interface. Yellow a white arrows mark defects in the $\mathrm{BaTiO}_{3}$ and $\mathrm{Sr}_{2} \mathrm{RuO}_{4}$ filn respectively. 
$\mathrm{BaTiO}_{3}$ over the same temperature range. ${ }^{6}$ This difference can lead to cracks and other defects in $\mathrm{BaTiO}_{3}$ films grown on $\mathrm{MgAl}_{2} \mathrm{O}_{4}$. A maximum thickness of $500 \mathrm{~nm}$ for crackfree $\mathrm{BaTiO}_{3}$ on $\mathrm{MgAl}_{2} \mathrm{O}_{4}$ has been reported. ${ }^{7}$ Thus, no suitable commercially available substrate with good lattice match exists for the growth of $\mathrm{BaTiO}_{3}$ at elevated substrate temperatures.

We propose the rare-earth scandates for the growth of $\mathrm{Ba}_{x} \mathrm{Sr}_{1-x} \mathrm{TiO}_{3}$ and other perovskites with lattice parameters in the 3.94-4.05 $\AA$ range as a class of substrates. The rareearth scandates were previously considered as possible laser crystals. ${ }^{8}$ The lattice parameters of several rare-earth scandates are listed in Table I. The rare-earth scandates are all orthorhombic at room temperature and isostructural with $\mathrm{GdFeO}_{3}$ (and $\mathrm{NdGaO}_{3}, \mathrm{SrRuO}_{3}$, etc.). $\mathrm{GdScO}_{3}$, for example, has lattice constants of $a=5.488 \AA, b=5.746 \AA$, and $c=7.934 \AA$ and belongs to the space group Pbnm. In this structure the (110) plane, spanned by [1 $\overline{1} 0]$ and [001] directions, provides a nearly square base with $c / 2=3.967 \AA$ and $\sqrt{a^{2}+b^{2}} / 2=3.973 \AA$. As is shown by the last two columns in Table I, the pseudocubic lattice constants of the scandates range from 3.94 to $4.05 \AA$. Therefore, these materials could be superior to existing substrate options for the epitaxial growth of perovkites with lattice parameters in this range. In this letter we evaluate the rare-earth scandates as useful substrates. One rare earth scandate that is currently commercially available, ${ }^{11} \mathrm{DyScO}_{3}$, is lattice matched to $\mathrm{SrRuO}_{3}$ better than $0.4 \%$. Here we focus on the growth of $\mathrm{BaTiO}_{3}$ on $\mathrm{GdScO}_{3}$ to obtain optically transparent films for use as waveguides and electro-optic devices. $\mathrm{GdScO}_{3}(110)$ has the lowest misfit to $\mathrm{BaTiO}_{3}$ of the rare-earth scandates which are now available commercially. ${ }^{11}$

$\mathrm{BaTiO}_{3}$ films were grown by pulsed laser deposition (PLD) using a $\mathrm{KrF}$ excimer laser (wavelength $248 \mathrm{~nm}$, pulse length $20 \mathrm{~ns}$, pulse frequency $10 \mathrm{~Hz}$, and a fluence of 2.5 $\left.\mathrm{J} / \mathrm{cm}^{2}\right) .{ }^{12}$ The substrates, single-side polished untwinned $\mathrm{GdScO}_{3}(110)$ single crystals $\left(5 \times 10 \times 0.8 \mathrm{~mm}^{3}\right)$, were placed $4 \mathrm{~cm}$ from the target on a $\mathrm{SiC}$ resistive heater. The $\mathrm{BaTiO}_{3}$ target was a sintered single-phase cylinder. $\mathrm{BaTiO}_{3}$ films were grown at substrate temperatures ranging from 600 to $800^{\circ} \mathrm{C}$ under an oxygen atmosphere of $2 \times 10^{-3}$ mbar. The crystallinity, epitaxial orientation relationship, and the stoichiometry of the films were investigated by Rutherford backscattering spectrometry and channeling (RBS/C), x-ray diffraction (XRD) $(\theta-2 \theta$ scans, rocking curve measurements, and $\phi$ scans) with a nonmonocromatic $\mathrm{Cu} K_{\alpha}$ source, and high-resolution transmission electron microscopy (HRTEM). The $\mathrm{BaTiO}_{3}$ film thickness was adjusted to $1 \mu \mathrm{m}$ to obtain low loss planar waveguides for optical characterization. The transparency and in-plane and out-of-plane refractive indices of the $\mathrm{BaTiO}_{3}$ films were measured using a prism coupling setup. ${ }^{13}$

The stoichiometry of the films was established by RBS/C. Comparison of simulated and measured spectra verified a 1:1 Ba:Ti ratio over the entire range of PLD growth conditions investigated. The observed channeling $\chi_{\min }=0.5 \%$ (Fig. 2) attests to the high crystalline quality of the $\mathrm{BaTiO}_{3}$. This value is lower than the lowest values for $\mathrm{BaTiO}_{3}$ films grown on $\mathrm{MgO}(100) .^{12}$ The lattice parameters determined from XRD analysis were $c=4.08 \pm 0.01 \AA$ (outof-plane) and $a=3.99 \pm 0.02 \AA$ (in-plane). These values are comparable to the lattice parameters observed in thick (and thus relaxed) epitaxial $\mathrm{BaTiO}_{3}$ films grown on $\mathrm{SrTiO}_{3} .{ }^{14} \mathrm{In}$ addition, the crystalline perfection of the films was corroborated by a full width at half maximum (FWHM) of the 002 reflection of $\mathrm{BaTiO}_{3}$ grown at $750^{\circ} \mathrm{C}$ of $\Delta \omega=0.13^{\circ}$. All of the $\mathrm{BaTiO}_{3}$ films had FWHMs below $\Delta \omega=0.4^{\circ}$, a typical value for $\mathrm{BaTiO}_{3}$ grown on $\mathrm{MgO}(100)$. Even for $\mathrm{BaTiO}_{3}$ films $1.5-\mu \mathrm{m}$-thick we observed no cracks. This is crucial for optical devices. This indicates that the thermal expansion coefficient of $\mathrm{GdScO}_{3}$ is reasonably well matched to the thermal expansion coefficient of $\mathrm{BaTiO}_{3} .{ }^{15}$

The improved microstructure of the $\mathrm{BaTiO}_{3}$ films was confirmed by HRTEM. Figure 3(a) shows a lowmagnification cross-sectional image revealing the typical mi-

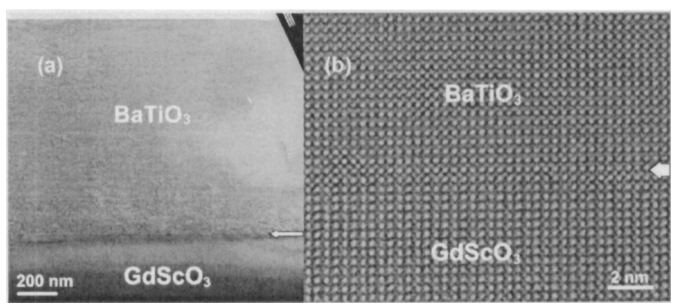

FIG. 3. (a) Cross-sectional TEM of a $1-\mu$ m-thick $\mathrm{BaTiO}_{3}$ film grown at $T_{\text {sub }}=6500^{\circ} \mathrm{C}$ on $\mathrm{GdScO}_{3}$. (b) Cross-sectional HRTEM of the interface of a $\mathrm{BaTiO}_{3}$ film grown at $T_{\text {sub }}=650{ }^{\circ} \mathrm{C}$ on $\mathrm{GdScO}_{3}$. The position of the $\mathrm{BaTiO}_{3} / \mathrm{GdScO}_{3}$ interface is indicated by the arrows. 
PRL 116, 106101 (2016)

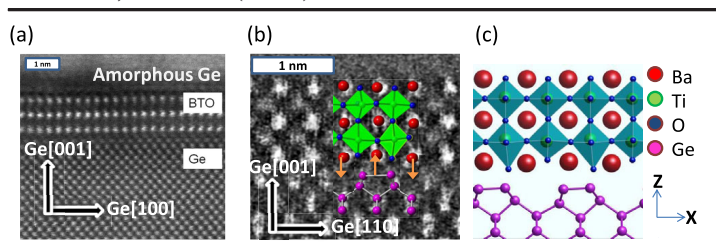

FIG. 1. (a) High angle annular dark field image of 2.5-uc BTO/Ge along the Ge[100] zone axis. (b) Superimposed on the TEM image taken along the Ge[110] zone axis is a model structure of the system determined from surface x-ray diffraction measurements. The red arrows indicate the rumpling of the interfacial $\mathrm{Ba}$ along the [001] axis. (c) DFT-predicted groundstate structure of the 2.5 -uc BTO/Ge system.

and $\mathrm{TiO}_{2}$. The low-temperature deposition leads to the formation of amorphous BTO as evidenced by a diffuse RHEED pattern. The wafer is then heated to $500^{\circ} \mathrm{C}$ in UHV whence the amorphous $\mathrm{BaO}$ and $\mathrm{TiO}_{2}$ react to form crystalline $\mathrm{BaTiO}_{3}$. To create the 5.5-uc sample, three additional monolayers of $\mathrm{BaO}$ and $\mathrm{TiO}_{2}$ are codeposited at a substrate temperature of $570{ }^{\circ} \mathrm{C}$. We note that the highertemperature deposition of the three additional layers for the 5.5-uc sample may lead to changes in the stoichiometry and interfacial structure as discussed below. Finally, for the 2.5-uc sample, a 7-nm-thick capping layer of amorphous Ge is deposited at room temperature to protect the ultrathin $\mathrm{BaTiO}_{3}$ film.

To characterize the structure of the BTO films, transmission electron microscopy measurements are carried out at Brookhaven National Laboratory. Figures 1(a) and 1(b) show high angle annular dark field images obtained along $\mathrm{Ge}[110]$ and $\mathrm{Ge}[100]$ zone axes, respectively, for the 2.5-uc BTO/Ge sample. The images indicate an abrupt interface with coherent atomic registry between the BTO film and the Ge. As explained below, the reduced contrast for the interfacial Ge layer is attributed to a Ge reconstruction and not to structural disorder in agreement with Fredrickson et al. where the interface is prepared starting with a Zintl phase consisting of half a ML of $\mathrm{Sr}$ on $\mathrm{Ge}(001)$ [20].

To confirm the ordered nature of the interface and the effect of the coupling across the interface, we use synchrotron x-ray diffraction measurements to determine the atomic structure of the system: atomic-scale structure of the samples are determined from the analysis of crystal truncation rods (CTRs) [35]. In addition to integer-order rods observed for both the 2.5- and 5.5-uc samples, for the 2.5-uc sample, superstructure reflections consistent with a $2 \times 1$ symmetry with respect to the bulk Ge are observed. From the periodicity of the superstructure reflections in the direction in reciprocal space perpendicular to the filmsubstrate interface, the $2 \times 1$ symmetry is determined to extend from the interface into the BTO film.

Atomic positions are derived from the measured CTRs and superstructure reflections by fitting using a chi-square

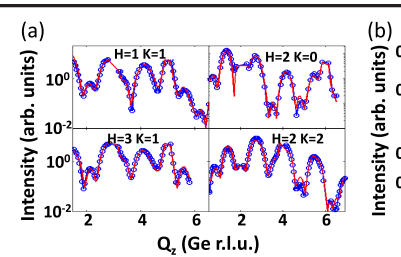

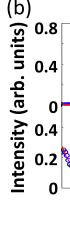

FIG. 2. (a) Integer-order (logarithı (b) superstructure (linear vertical scale measured (blue circles) for a 2.5-uc BT using molecular beam epitaxy. The BTO/Ge interface shown in Fig. 1(b) (red lines) to the measured data.

minimization genetic fitting algor [36]. For this analysis, we conside of two $90^{\circ}$ rotational domains that a steps on the $\mathrm{Ge}(001)$ surface. Figu measured diffraction intensities al order rods, respectively, and their converged 2.5-uc sample. The XR of the 2.5-uc sample is shown supe image of the same sample in Fig. 1(1 the XRD structure are well reprodu i.e., the rumpling of a full monolay and the displacement of $\mathrm{Ti}$ along th The $\mathrm{O}$ positions, which are not resol are determined from the $\mathrm{x}$-ray CTF

For the 2.5-uc sample, the int displaced in plane from their bulk $\mathrm{p}$ rows along the [010] direction. A n direct contact with the topmost $\mathrm{Ge}$ interfacial $\mathrm{BaO}$ occurs in respons dimerization doubling the size of th [100] direction. The $\mathrm{BaO}$ displacem ments of the $\mathrm{Ti}$ and $\mathrm{O}$ ions resulti entire film relative to the expecte Along [100], the alternate contractic $\mathrm{TiO}_{6}$ octahedra is consistent with tortion, absent in the bulk phase distortions are observed to decay in growth.

The stability of the observed s sample is confirmed from first-princ theory (DFT) calculations (see the [37] for full details). The groun coordinates of the BTO/Ge systen retically and are found to be in experimentally measured structure

In contrast to the 2.5 -uc film, sample the CTR analysis and the ab Bragg reflections show that the ent interfacial Ge have a $1 \times 1$ symmetr has lattice constants $a=b=3.99$ agreement with those of bulk tetra 

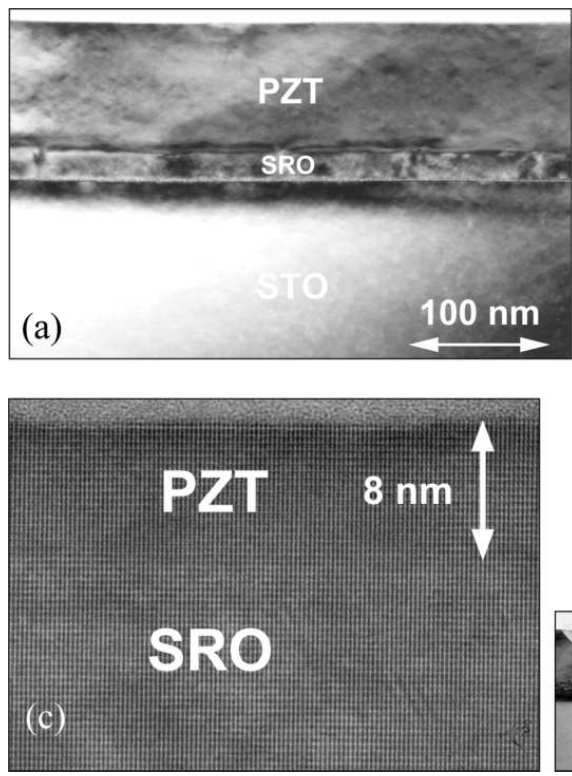

Figure 2. TEM investigations of the $\mathrm{PbZr}_{02} \mathrm{Ti}_{0.8} \mathrm{O}_{3} / \mathrm{SRO} / \mathrm{STO}$ heterostructures. The images are: a) cross-sectional TEM image of a defect-free PZT film ( $t=90 \mathrm{~nm})$ on top of an SRO film $(t=20 \mathrm{~nm})$ grown on vicinal STO $(001)$, b) electron diffraction pattern of this heterostructure, c) high-resolution TEM (HRTEM) image of an ultrathin PZT film $(t=8 \mathrm{~nm})$, d) Z-contrast STEM image showing the sharp and dislocation-free interface between the PZT and SRO layers of such a heterostructure, and e) cross-sectional TEM image of a defective PZT film $(t=120 \mathrm{~nm})$ on top of an SRO film $(t=20 \mathrm{~nm})$ grown on vicinal STO (001).
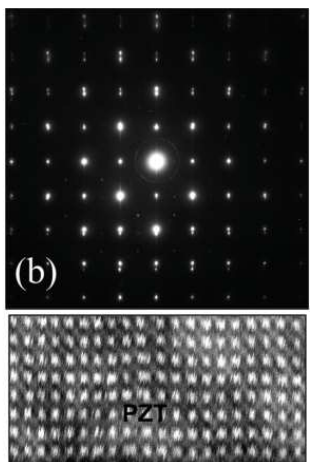

SRO

(d)
$\mathrm{SrTiO}_{3}$

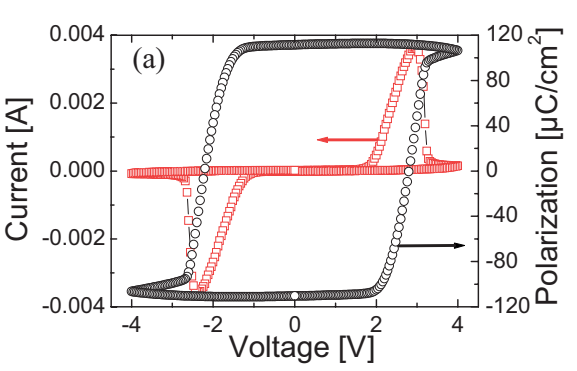

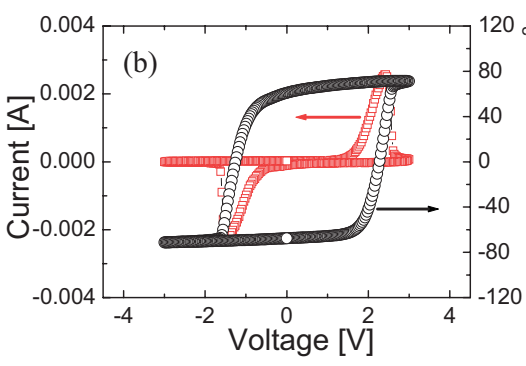
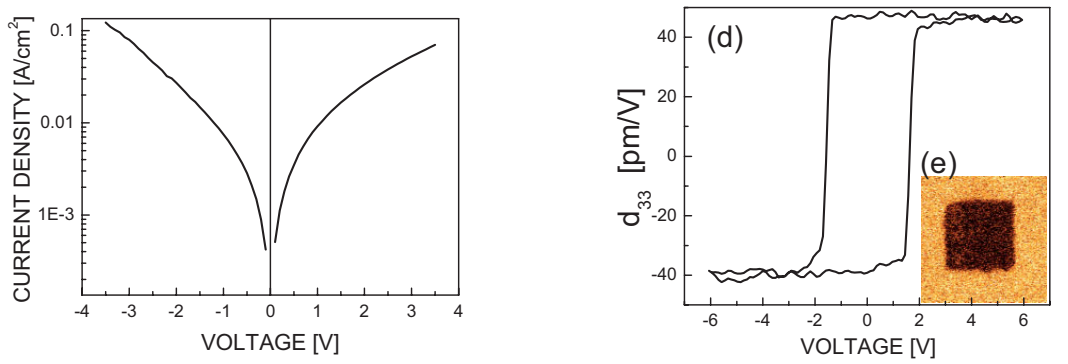

Figure 3. Ferroelectric and piezoelectric investigations of the $\mathrm{PbZr}_{0.2} \mathrm{Ti}_{0.8} \mathrm{O}_{3} / \mathrm{SrRuO}_{3} / \mathrm{SrTiO}_{3}$ heterostructures. Ferroelectric hysteresi at $1 \mathrm{kHz}$ are shown for a) a defect-free PZT film (remnant polarization, $\left.P_{\mathrm{r}}=105<\mathrm{M}+\rightarrow 5 \mu \mathrm{C} \mathrm{cm}^{-2}\right)$ and b) a defective film $\left(P_{\mathrm{r}}=65\right.$ current density versus voltage measured on the defect-free PZT layer is shown in (c) and local piezoelectric coefficient $\left(d_{33}\right)$ hyst layer is displayed in (d). The inset (e) is a $1 \mu \mathrm{m} \times 1 \mu \mathrm{m}$ piezoresponse image, where the center area $(0.5 \mu \mathrm{m} \times 0.5 \mu \mathrm{m})$ has switch being scanned with the tip under DC bias. No backswitching was observed after a time as long as $71 \mathrm{~h}$.

diffraction (XRD), being th ure only the volume fract TEM was not emp work. However, XRD was tain the possible existenc structural defects, such as heteroepitaxial films. TEM are therefore mandatory wh quiredion of the crystalline relationship between $P_{\mathrm{S}}$ an tragonal PZT. From the curve (shown in Fig. 5 of value of $P_{\mathrm{S}} \approx 110 \mu \mathrm{C} \mathrm{cm}^{-2}$ w $c / a \approx 1.058$.

The ferroelectric and pie erties of our PZT films we n detail. Ferroelectric pc shown in Figure 3a. The were performed on the d film (90 nm thick) shown in polarization hysteresis curv shape, remnant polarization as $P_{\mathrm{r}} \approx 105 \pm 5 \mu \mathrm{Ccm}^{-2}$, and to the $P_{\mathrm{S}}$. The polarization weak frequency dependence This value is, to the best of 
(a)

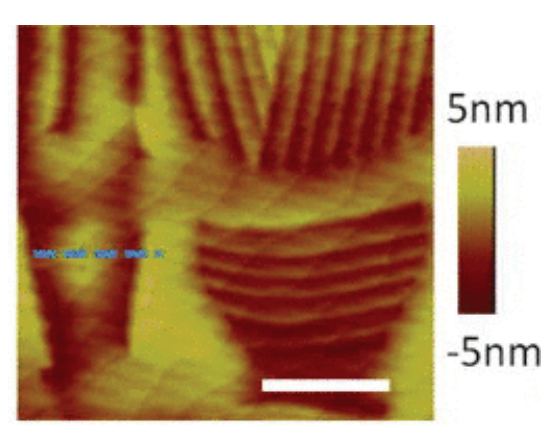

(b)

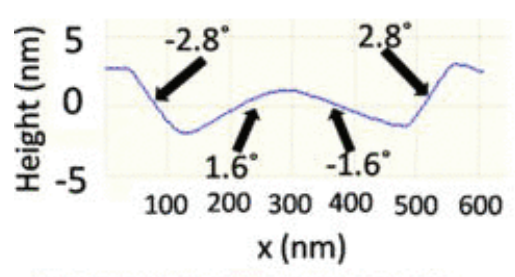

(d)

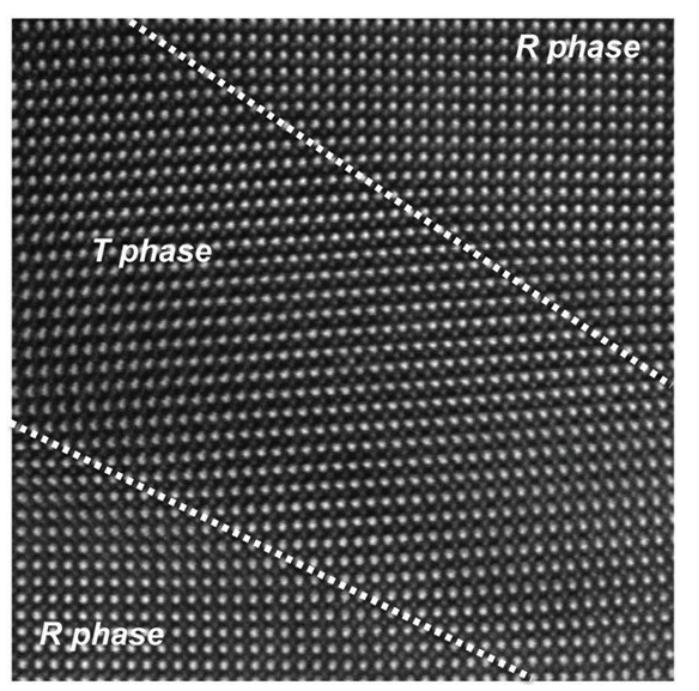

(c)

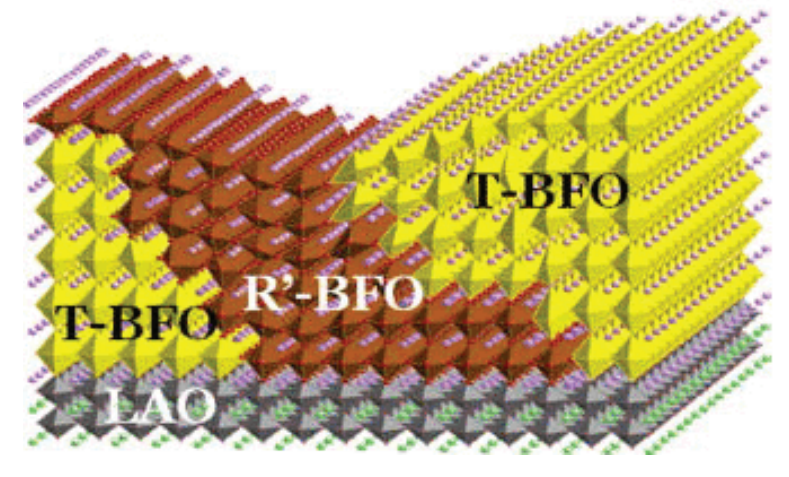

(e)

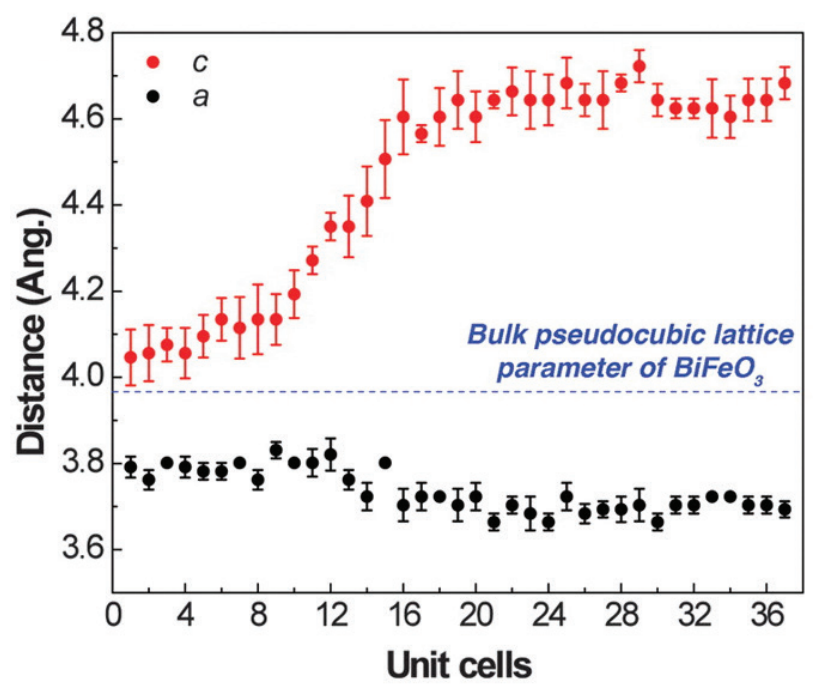


study, resulting in the growth of the orthorhombic epitaxial film on a single-crystal substrate by direct growth from vapor. A similarly meta-stable orthorhombic phase in solid solution has been achieved in studies of quenched samples from molten materials, which are prepared by the arc melting of sol-gel derived powders. ${ }^{18}$ These results including the present one imply that the formation of a solid solution is one of the key factors in obtaining the orthorhombic phase in $\mathrm{HfO}_{2}$-related systems. It is worth mentioning that the postcrystallization of the amorphous film, by which the ferroelectric orthorhombic films are obtained, is one way to obtain a solid solution. Another possibility to obtain orthorhombic phase is due to epitaxial strain. The theoretical calculation by Materlik et al. indicates that the epitaxial strain is possible to stabilize ferroelectric orthorhombic phase. ${ }^{21}$

In order to elucidate that the orthorhombic film in this study is ferroelectric orthrohombic phase, we performed ABF-STEM imaging. The ABF-STEM imaging has advantages to observe light element compared to other STEM observation, like HAADF-STEM. Our observed ABF-STEM image and multislice simulation for considerable three orthorhombic phases are shown Fig. 4. Black spots indicate hafnium atomic columns while grey spots indicate the oxygen atomic columns. The tail like grey contrast of two oxygen atomic columns, which is a characteristic pattern consistent to the polar structure along $c$-axis, can be observed for the observed ABF-STEM image and simulation for the Pca2 structure, whereas spot like light grey contrast located at center of for black area (hafnium column) are observed in simulation for the $\mathrm{Pbcm}$ and $\mathrm{Pbca}$ structure, which have inversion center. These results suggest that the present orthorhombic epitaxial film has polar structure, implying the possibility to exhibit ferroelectricity. However, further investigation is needed to clarify the ferroelectricity in epitaxial films.

The structural phase transition provides strong evidence of ferroelectricity because most polar materials with switchable polarization show a phase transition from high-symmetry paraelectric to low-symmetry ferroelectric phases with decreasing temperature. Fortunately, 110 diffraction can be observed in the orthorhombic phase, which is considered to be a ferroelectric phase, while such diffraction is not seen in the tetragonal phase, which is expected to be paraelectric. Therefore, 110 diffraction is a useful probe for detecting this phase transition. Figure 5(a) shows the XRD pattern with inclination angle of $45^{\circ}$ measured at various temperatures for $0.07 \mathrm{YO}_{1.5}-0.93 \mathrm{HfO}_{2}$ film, which has an orthorhombic phase at room temperature as shown in Fig. 3(b). In addition to the strong peak around the $2 \theta$ angle of $50^{\circ}$, the weak 110 peak at around $24.5^{\circ}$ can be observed in the pattern measured at room temperature. Note that the peaks at around $29^{\circ}$ and $47^{\circ}$, which are marked by star symbols in the figure, came from the carbon dome of the measurement set-up. The intensity of the 110 peak decreased with the increase in temperature, and disappeared above $500{ }^{\circ} \mathrm{C}$. Figure 5(b) summarizes the 110 peak intensity as a function of temperature. A parabolic curve of intensity against temperature was observed; similar temperature-dependent behaviors are often found through structural phase transitions. ${ }^{22}$ Thus, Fig. 5(b) strongly suggests a phase transition from tetragonal to orthorhombic. From the figure, the phase-transition temperature can be estimated at around $450^{\circ} \mathrm{C}$. This temperature is
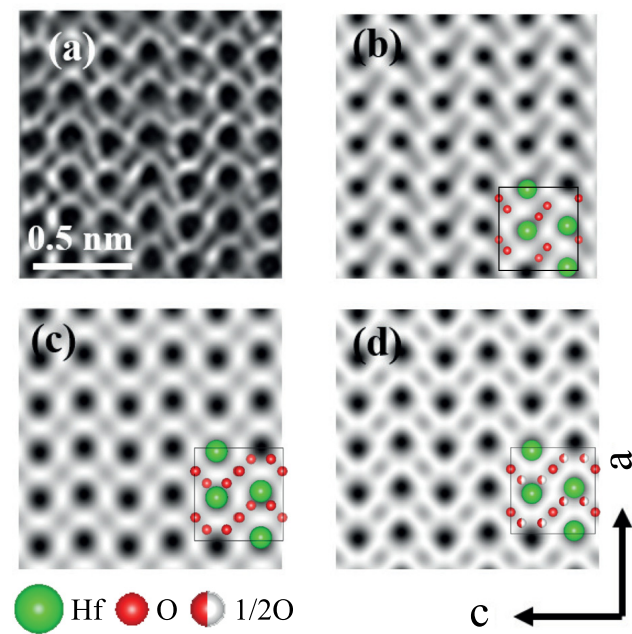

FIG. 4. (a) ABF-STEM image observed for $0.07 \mathrm{YO}_{1.5}-\mathrm{HfO}_{2}$ epitaxial film. Simulated ABF-STEM images for (b) polar $\mathrm{Pca}_{1}$, (c) non-polar Pbca, and (d) $\mathrm{Pbcm}$ with the corresponding column arrangement of $\mathrm{Hf}$ and $\mathrm{O}$ arrangements.

significantly higher than the reported maximum temperature of $200^{\circ} \mathrm{C}$, where ferroelectricity has been confirmed by polarization-electric-field $(P-E)$ hysteresis measurement. ${ }^{23}$ This implies that the ferroelectric phase transition temperature is
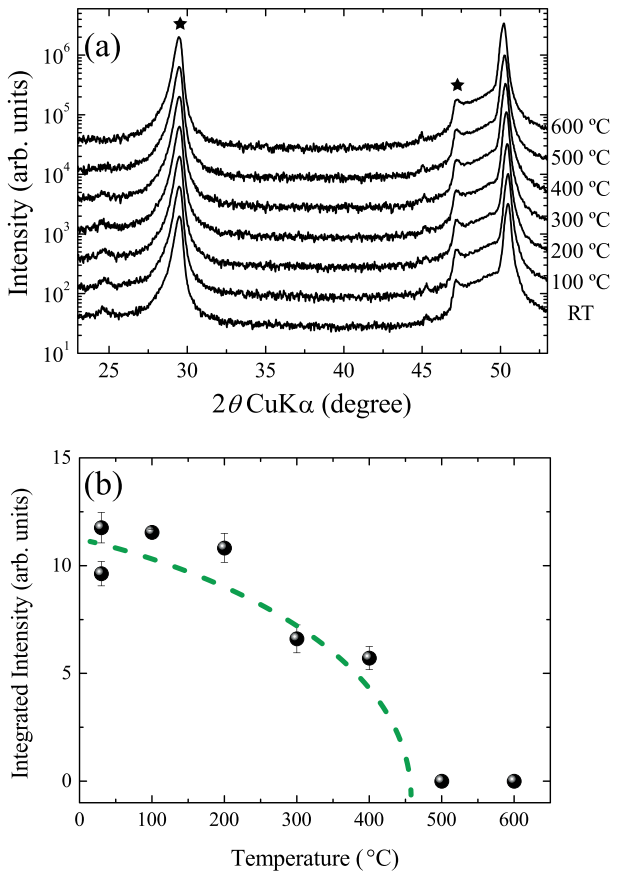

FIG. 5. (a) The XRD patterns with inclination angle of $45^{\circ}$ observed for $0.07 \mathrm{YO}_{1.5}-0.93 \mathrm{HfO}_{2}$ film measured from room temperature to $600^{\circ} \mathrm{C}$. (b) The integrated intensity of the 110 super-spot of $0.07 \mathrm{YO}_{1.5}-0.93 \mathrm{HfO}_{2}$ film as a function of temperature. 
to vary as a function of strain even when the dipole moment does not change (see Fig. 1) [9,27]. This pure volume effect is included in the improper but not in the proper piezoelectric response. However, the two are trivially related by the spontaneous polarization (in the case of $c_{33}$ the proper is equal to the improper piezoelectric response; for $c_{31}$ the proper piezoelectric response is given by the improper value plus the spontaneous polarization) $[9,27]$. Here we report the improper piezoelectric response, which is used in Eqs. (1) and (2), since they refer to the total change in spontaneous polarization. We point out that the improper piezoelectric constant corresponding to the spontaneous polarization is well defined and does not depend on the specific branch used in the evaluation of this quantity.

The improper piezoelectric constants and Poisson ratios for the five systems are given in Table III. Note that for the tetragonal systems the fourfold axis is used as the $z$ direction of our coordinate system, whereas for the rhombohedral systems the threefold axis is used. It is evident that the effective piezoelectric constants $c_{\text {eff }}$ describing the effect of epitaxial strain (to linear order) on the spontaneous polarization are much larger for $\mathrm{BaTiO}_{3}$ and $\mathrm{PbTiO}_{3}$ than for the other three systems. This is mainly due to the larger $c_{33}$ in $\mathrm{BaTiO}_{3} / \mathrm{PbTiO}_{3}$ but also due to the smaller Poisson ratio of the $P 4 \mathrm{~mm}$ structures [28]. The Poisson ratios are comparable for systems with the same structural symmetry. In $P 4 \mathrm{~mm} \mathrm{BiFeO}_{3}$ the smaller Poisson ratio leads to a slightly increased $c_{\text {eff }}$ compared to $R 3 c$ $\mathrm{BiFeO}_{3}$ although the piezoelectric constant $c_{33}$ is similar for both symmetries. Another observation is that, although $c_{33}$ is smaller in $R 3 c \mathrm{BiFeO}_{3}$ than in $\mathrm{LiNbO}_{3}, c_{\text {eff }}$ is larger due to the different sign of $c_{31}$.

Figure 2 compares the directly calculated change in spontaneous polarization caused by epitaxial strain with

TABLE III. Improper piezoelectric constants $c_{33}, c_{31}$, and $c_{\text {eff }}$ (in $\mu \mathrm{C} / \mathrm{cm}^{2}$ ) as well as the Poisson ratio $n$ for the various systems. References are given for values not calculated in this work.

\begin{tabular}{lcrlr}
\hline \hline & $c$ & \multicolumn{1}{c}{$n$} & \multicolumn{1}{c}{$c_{\text {eff }}$} \\
\hline $\mathrm{BaTiO}_{3}$ & 670 & 30 & $0.65[4]$ & -971 \\
$\mathrm{PbTiO}_{3}$ & 586 & 103 & $0.58[6]$ & -804 \\
$\mathrm{BiFeO}_{3}(R 3 c)$ & 213 & 50 & 1.15 & -85 \\
$\mathrm{LiNbO}_{3}$ & 124 & -75 & 1.5 & -233 \\
$\mathrm{BiFeO}_{3}(P 4 m m)$ & 203 & -15 & 0.65 & -342 \\
\hline \hline
\end{tabular}

Table III for all five systems. One can see that the line approximation of Eq. (2) works well for all systems up strains of $\pm 3 \%$. Even in the case of $\mathrm{BaTiO}_{3}$ and $\mathrm{PbTiC}$ where we have used data from two different sources, $\mathrm{t}$ agreement between the polarization calculated usil Eq. (2) and the directly calculated values is remarkab good. For the systems where all values are calculated in th present work the agreement is extremely good.

The present study shows that the polarization respon to epitaxial strain for experimentally relevant strain valu can be described in terms of the piezoelectric and elast constants of the unstrained system. Two further observ tions can be made. First, the appearance of two differe terms in Eq. (2) with potentially opposite signs makes possible that a system can in principle have rather lar piezoelectric constants, but that due to partial cancellatic $c_{\text {eff }}$ is rather small. Second, a large value of $c_{33}$ eventually be suppressed by a large Poisson ratio. Othe wise, if no partial cancellation occurs, a system with a lar piezoelectric response will in general also show a strol response of the spontaneous polarization to epitaxial strai

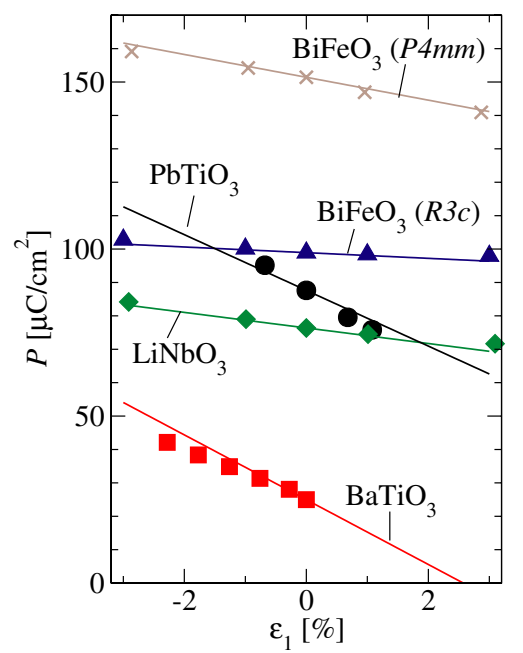

FIG. 2 (color online). Spontaneous polarization $P$ for seve ferroelectrics as a function of epitaxial strain $\epsilon_{1}$. Symbc represent directly calculated values, lines are calculated usi Eq. (2), and the effective piezoelectric constants are fro Table III. Directly calculated values for $\mathrm{BaTiO}_{3}$ and $\mathrm{PbTi}$ are taken from Refs. $[4,6]$, respectively. 

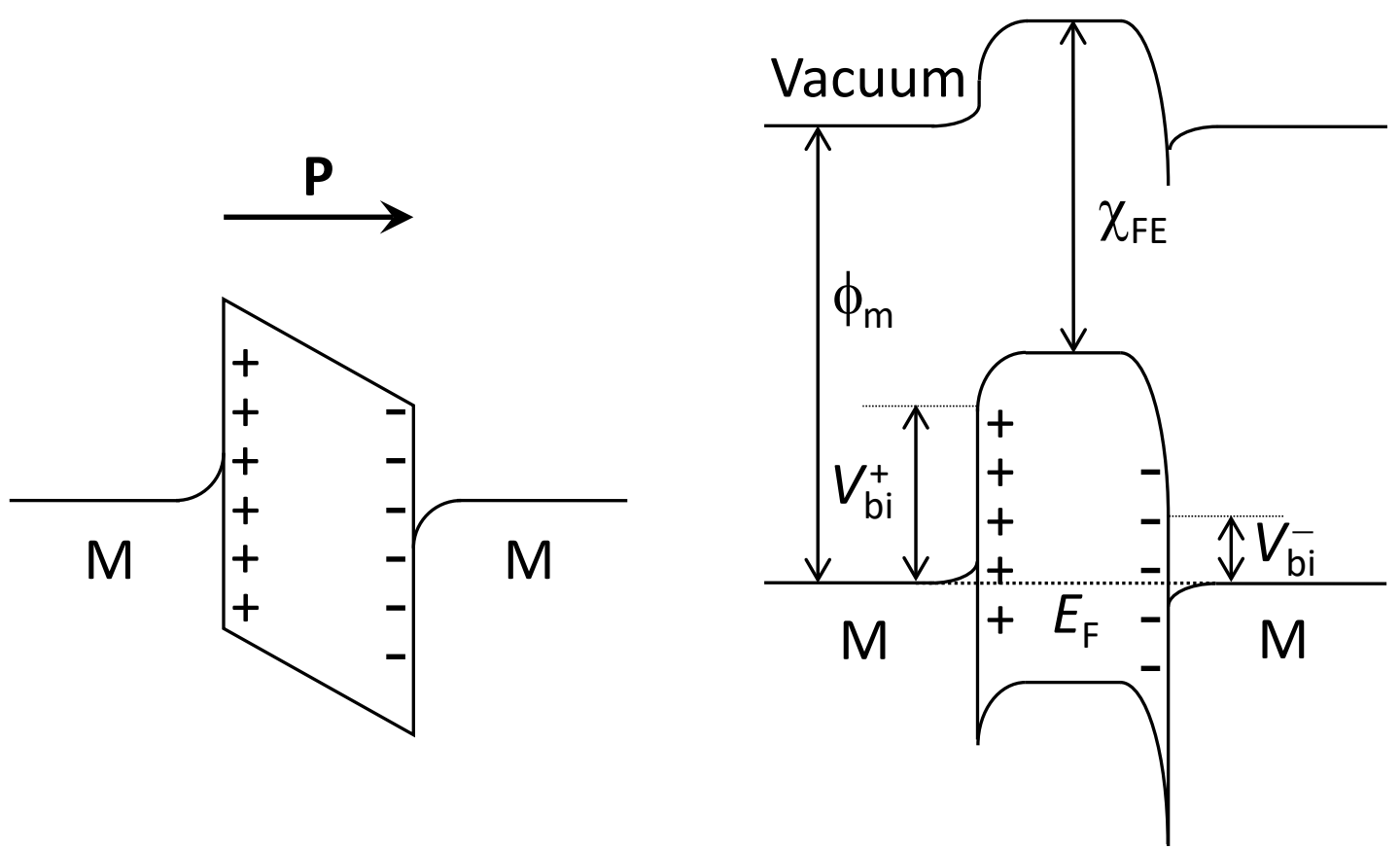

(a)

(b) 


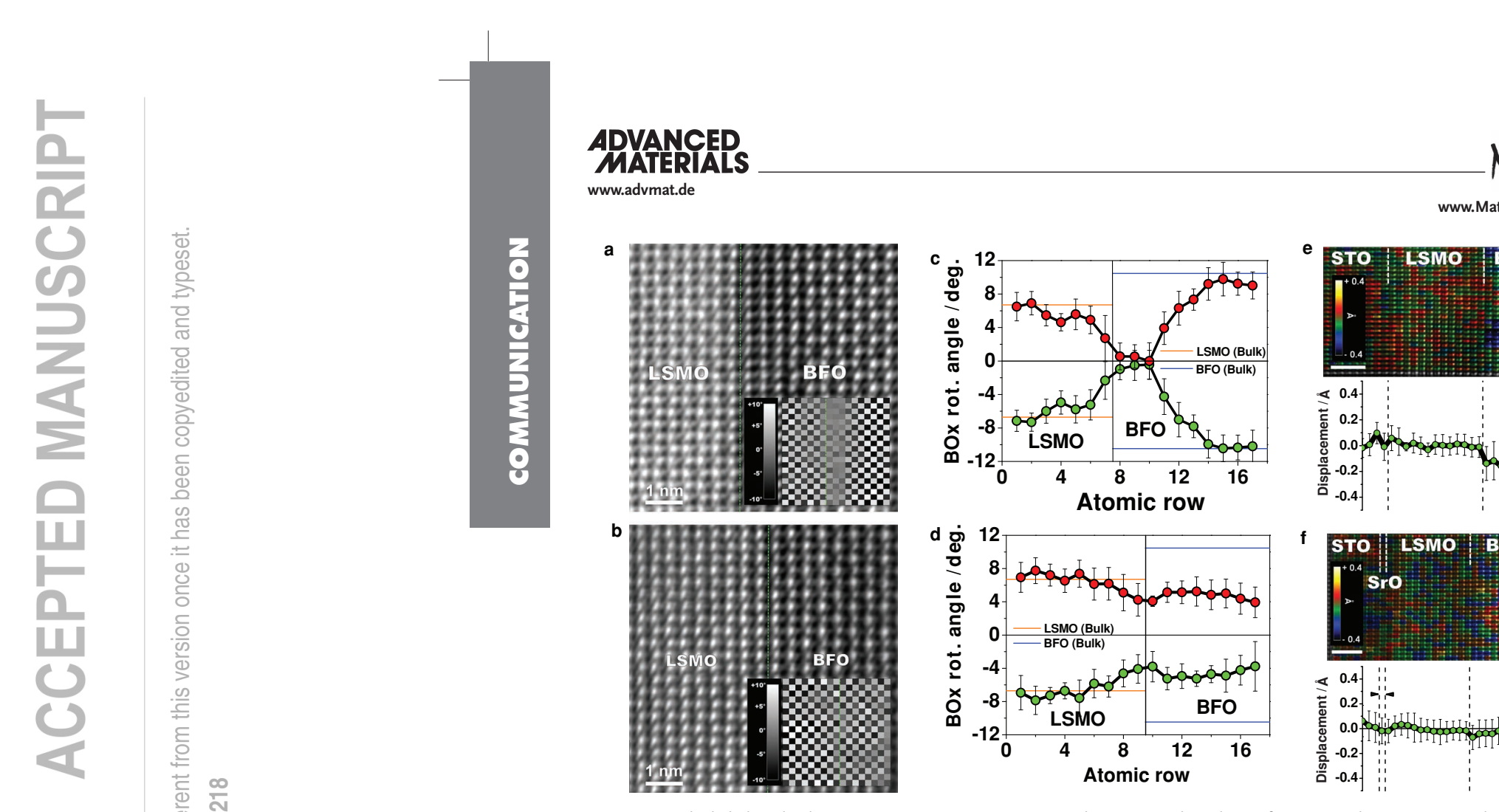

Figure 2. Octahedral tilt and polarization mapping: BF STEM images taken in optimal conditions for oxygen column contrast in the BFO film structures grown on: a) $\mathrm{MnO}_{2}$-terminated LSMO and b) ( $\mathrm{La}, \mathrm{Sr}$ ) O-terminated LSMO. The resulting octahedral tilt maps (ins erboard pattern of tilt angles. c,d) Line profiles of octahedral tilts averaged over vertical rows of the respective tilt maps in (a) and (t each graph represent tilt angles in the bulk BFO and LSMO. e,f) Out-of-plane ( $d z)$ displacement maps and the corresponding average B-site cations in both BFO thin film heterostructures, showing the polarization behavior (data given on the same scale for both films overlaid on the respective ADF STEM images. The scale bars are $2 \mathrm{~nm}$.

according to definition by Balashova and Tagantsev ${ }^{[40]}$ (alternative definitions, such as characteristic $P-E$ double loop, do not provide any structural criteria and are insufficiently specific (e.g., ferroelectrics above $T_{c}$ can also show double loops)). Confirming this classification, small (close to detection limit) antipolar in-plane displacements attributed to modulations in the Bi atomic columns were observed in structure B (Figure S5 in the Supporting Information); in the structure A, antipolar displacements were only observed next to the small regions with incomplete suppression of octahedral tilts (Figure S4 in the Supporting Information), suggesting that the nonpolar phase coincides with a metastable AFE phase of BFO with different octahedral rotations. These results suggest that the heterointerface termination mediates the interplay between local cation displacements, electric polarization and octahedral rotation behaviors.

The structural distortions and interface-induced nonpolar phase extend for large distances (3-15 nm) into the film. At these length scales, ferroelectric properties and bias-induced phase transitions between nonpolar and polar states may be indirectly accessed by piezoresponse force microscopy (PFM). ${ }^{[41-43]}$ We note that direct measurements of the antiferroelectric-like hysteresis loop by PFM are complicated even for prototypical AFE materials such as $\mathrm{PbZrO}_{3} \cdot{ }^{[44]}$ In many cases, materials showing constricted $P-E$ macroscopic hysteresis loops yields FE like hysteresis loops on the nanoscale. ${ }^{[45]}$ From STEM data, the materials studied here are the mixture of nanometer scale mesoscopic FE and AFE regions, and hence measured PFM signal will contain contributions from both st be dominated by FE response (since the respon phase is zero).

To explore the domain structure and polarizat in these systems systematically, we performed I domain writing, and hysteresis loop measureme ture A, the PFM images indicate the presence of $c$ like domain structure formed by $50-80 \mathrm{~nm}$ size all film thicknesses, Figure $3 \mathrm{a}-\mathrm{c}$, in agreement studies for similar films. ${ }^{[46,47]}$ For structure B in t (Figure 3d), however, the regular domain struc and the images show considerably reduced aver of the PFM signal. This data is consistent with nanoscale polar regions with random polarizati and nonpolar regions (despite the high quality o Large regular domain patterns are observed only $100 \mathrm{~nm}$ films (Figure 3e,f). The morphology of $t$ is markedly different from the structure A serie rounded. X-ray diffraction studies (see Supporting also confirm the absence of domain walls in the ture B film.

This field-induced transitions and polarizat in these materials are explored through the switching experiments illustrated in Figure 4. V exhibit classical switching behavior (similar PFM upward-polarized, downward-polarized, and pri 180 phase change at the domain boundaries), th with structure B shows more complex behav 
is a schematic diagram showing the position of the domain structure and of the dislocations in this area of the sample. HAADF images with higher magnifications in Figs. 2(d) and 2(e) display in more detail the areas marked in Fig. 2(c). The spots with brighter contrast represent $\mathrm{Pb}$ columns, due to its higher atomic number. Figure 2(c) reveals that the $90^{\circ}$ domain walls are mainly pinned by misfit dislocation pairs, with Burgers vector along [100] on the upper side and along
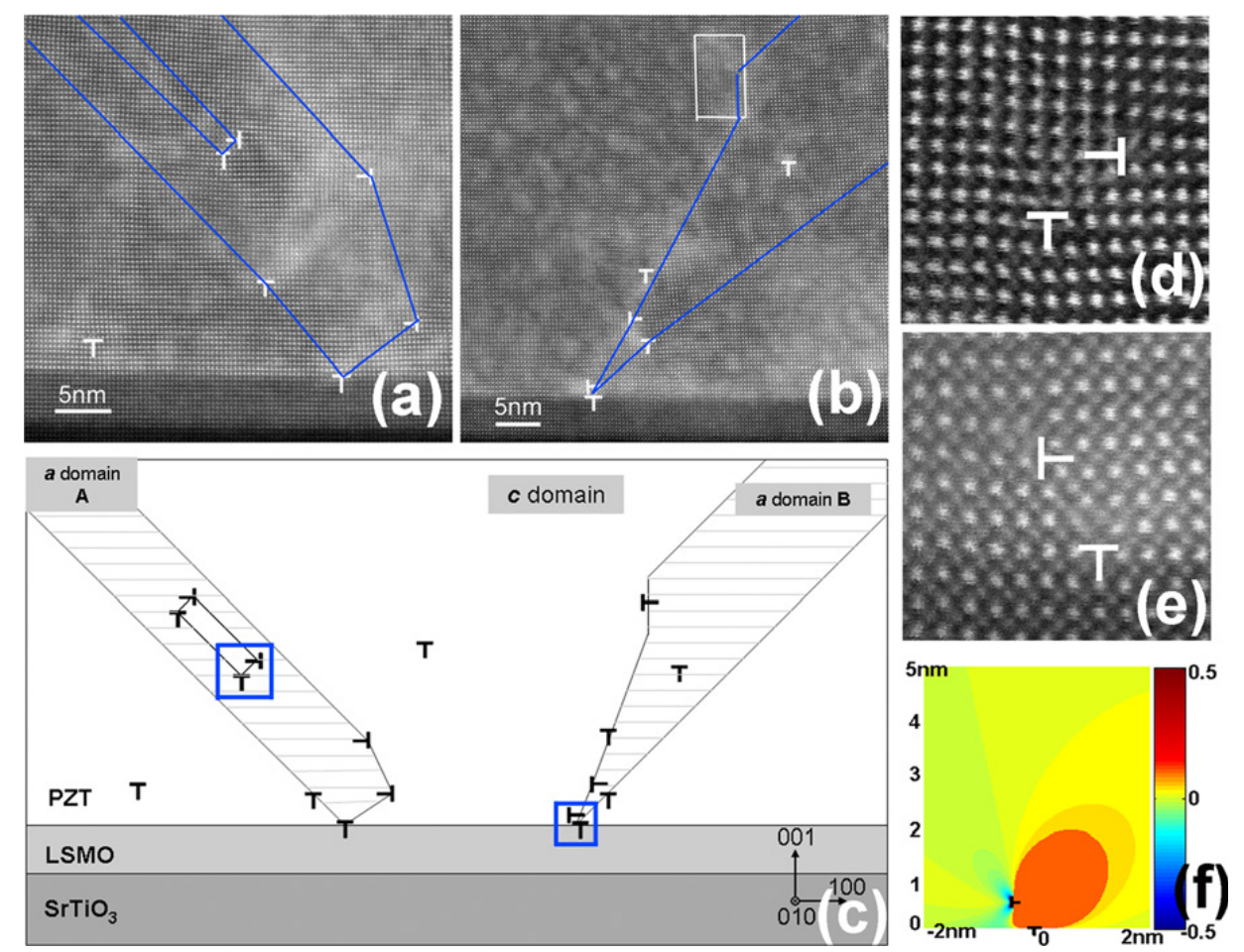

FIG. 2. (Color online) (a) and (b) STEM ADF images along the [010] zone axis of the A and B domains, respectively. Domain walls are marked by the solid lines. (c) Schematic diagram of the pinning of the domains A and B in (a) and (b). (d) and (e) STEM ADF images taken at higher magnifications on the areas marked in (c). (f) Calculated spatial variation of the elastic field $\epsilon_{\mathrm{dis} / x x}^{c}$ in the PZT film. The interface between PZT and substrate is at $y=0$.

Downloaded 13 Sep 2011 to 192.33 .126 .163 . Redistribution subject to All 


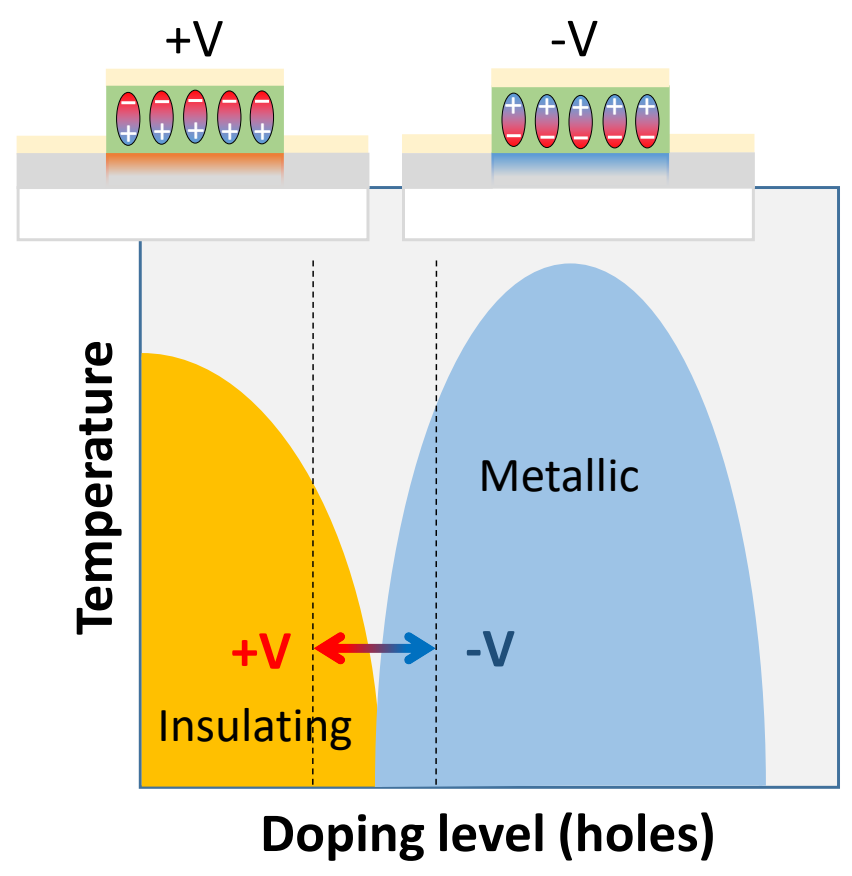



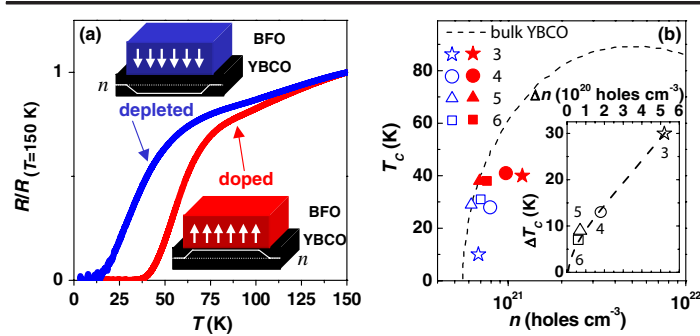

FIG. 2 (color online). (a) $R(T)$ normalized to the resistance at $T=150 \mathrm{~K}$ of a heterostructure with $t_{\mathrm{YBCO}}=3$ u.c. measure with $J=1.7 \mathrm{kA} \cdot \mathrm{cm}^{-2}$ for two neighboring areas in which the BFO polarization points down [left-hand (blue) curve] and up [right-hand (red) curve]. The inset shows schematics of the charge carrier depletion or accumulation induced in the YBCO by the ferroelectric polarization (white arrows). (b) $T_{C}$ vs charge carrier density $n$ for samples with different $t_{\mathrm{YBCO}}$ (see legend in u.c.), for the "depleted" (hollow symbols) and "doped" (solid symbols) states. Inset: modulation of the $T_{C}$ vs carrier density modulation $\Delta n$. The labels indicate $t_{\mathrm{YBCO}}$ in u.c.

the polarization is up (doped state). A large difference between the critical temperatures $\Delta T_{C}$ is observed.

Figure 2(b) shows $T_{C}$ vs the charge carrier density $n$ for samples with different $t_{\mathrm{YBCO}}$ (see the legend). For each sample, $n$ and $T_{C}$ were measured in the same sample area (i.e., between a fixed pair of voltage probes) (i) for a down polarization (depleted state, hollow symbols) and (ii) after the polarization had been switched up at room temperature (doped state, solid symbols). $T_{C}$ is defined with the criterion $R\left(T_{C}\right)=0.1 R_{N}$, with $R_{N}$ denoting the normal-state resistance at the onset of the transition. $n$ was obtained from Hall-effect experiments in the normal state just above the superconducting transition onset. By taking $T_{C}(n)$ for bulk YBCO as a reference [dashed line in Fig. 2(b)], one can see that, in all cases, the $\Delta T_{C}$ induced upon ferroelectric switching is accompanied by a consistent modulation of the carrier density, $\Delta n$. This allows for an understanding of the correlation between $\Delta n$ and $\Delta T_{C}$, shown in the inset of Fig. 2(b).

We observe [inset of Fig. 2(b)] that the carrier density modulation $\Delta n$ decreases for increasing YBCO thickness $t_{\mathrm{YBCO}}$, as it is expected [1,6,7]. This is, on the one hand, because the volume (and consequently the absolute number of carriers $N$ ) under the ferroelectric gate is proportional to $t_{\mathrm{YBCO}}$. Therefore, for a fixed $\Delta N$ (given by the polarization $P$ ), the thicker the $t_{\mathrm{YBCO}}$, the smaller the density modulation $\Delta n$. In addition, and because $t_{\mathrm{YBCO}}$ is, in all the samples, longer than the Thomas-Fermi screening length $\lambda_{\mathrm{TF}} \sim 1$ u.c. [7], the electrostatic doping efficiency is expected to gradually lower as $t_{\mathrm{YBCO}}$ increases $[1,2,7]$. Thus, the largest $\Delta n=5.2 \times 10^{20} \mathrm{~cm}^{-3}$ and $\Delta T_{C} \sim 30 \mathrm{~K}$ are observed for the thinnest $t_{\mathrm{YBCO}}=3$ u.c. Note that this $\Delta T_{C}$ is much in excess of previous realizations of ferroelectric modulation of superconductivity [8] in which $\Delta T_{C} \sim 7 \mathrm{~K}$. Because of th ization causes here a complete swit tivity: at temperatures $\sim 35 \mathrm{~K}$, the normal or in the superconducting $\mathrm{s}$ ferroelectric polarization [see Fig. that $\mathrm{BFO}$ produces a large sheet $\mathrm{c}$ $\Delta n_{\mathrm{sh}}=\Delta n t_{\mathrm{YBCO}}$, as compared to particular, while $\Delta n_{\mathrm{sh}} \sim 1.2 \times 10$ tained with $\operatorname{Pr}(\mathrm{Zr}, \mathrm{Ti}) \mathrm{O}_{3}$ in superco lar thickness [8], $160 \%$ of that can $\left(\Delta n_{\mathrm{sh}} \sim 1.9 \times 10^{14}\right.$ holes $\cdot \mathrm{cm}^{-2}$ Note finally that this is only 2 ? $\Delta n_{\mathrm{sh}}^{\max }=2 P / e \sim 8 \times 10^{14}$ holes $\cdot c$ $P \sim 65 \mu \mathrm{C} \cdot \mathrm{cm}^{-2}$ for BFO alon (Ref. [25]). Although screening b might play a role in diminishing $\Delta n_{\mathrm{sh}}<\Delta n_{\mathrm{sh}}^{\max }$ is to a larger extent the studied samples $[1,2,7]$. In con modulations could be expected usin $t_{\mathrm{YBCO}}<3$ u.c.

We verified that the field effect persistent at least for 8 days and $t$ training effects, the $T_{C}$ shift is essen expected [8]. This was done by perf the BFO polarization was alternat down and the $R(T)$ subsequently $\mathrm{m}$

We show in what follows that described above can be used to prod lation of the superconducting cond created periodic arrays of ferroelec done via a two-step process. First, voltage probes was homogeneousl state) using the AFM as descri sequently, $-8.5 \mathrm{~V}$ pulses $(20 \mathrm{~ms}$ in applied between the YBCO layer a odically in space along the $x$ and $y$ reversed the polarization, creating a domains ("dots" with diameters $\varnothing$ the polarization points down. PFM arrays are displayed in Fig. 3(a) (array B). In both cases, the array $\mathrm{c}$ a parallelogram with sides $a \neq b$. A ferroelectric patterns, the $T_{C}$ is con which the ferroelectric has a homog

Evidence for the nanoscale modu tivity induced by the ferroelectric a the mixed-state magnetoresistanc curves in Figs. 3(c) and 3(d) show $H$ (applied perpendicular to the arrays $\mathrm{A}$ and $\mathrm{B}$, respectively. In b current $J$ is applied parallel to the a For the array A [Fig. 3(c)], two symmetrically around $H=0$ at th 40 Oe. This behavior must be comp magnetoresistance exhibited by the 

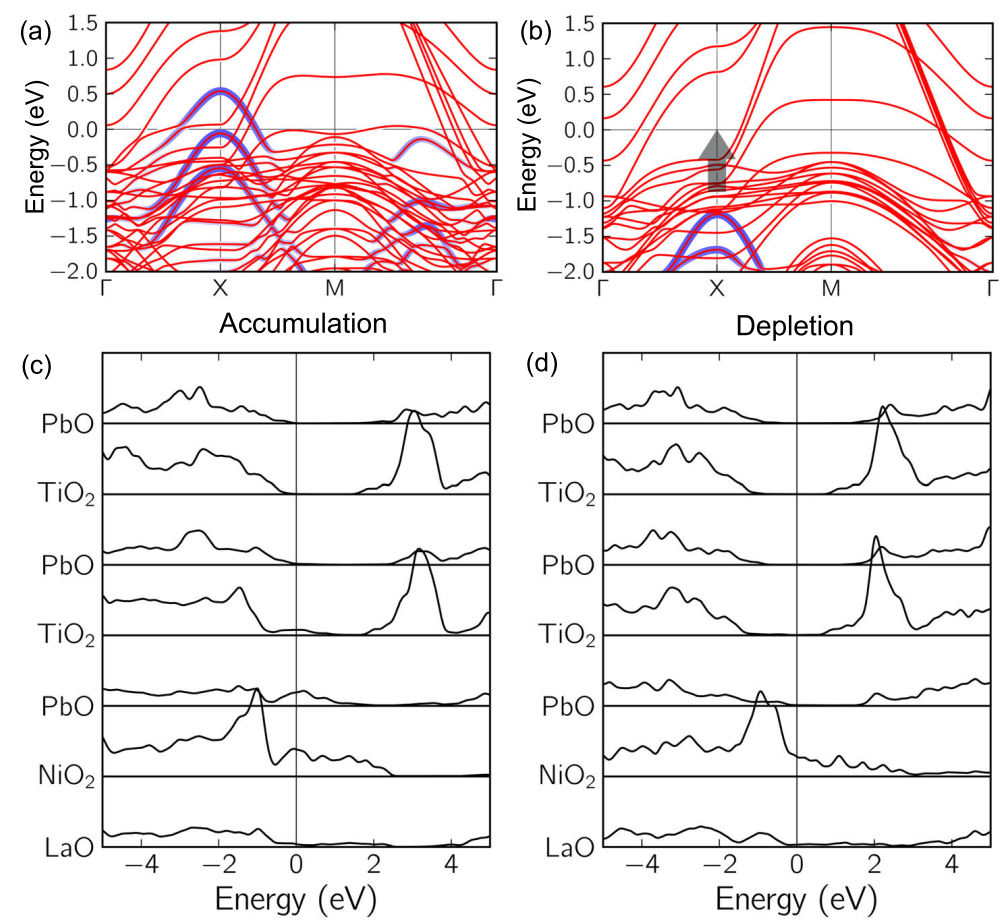

FIG. 3. Band structure of $\mathrm{PTO} / \mathrm{LaNiO}_{3}$ strained to the theoretical in-plane lattice constant of $\mathrm{LaAlO}_{3}$. Band structure fo (a) accumulation and (b) depletion, where the zero of energy is the Fermi level in each case and a $(1 \times 1)$ interfacial unit cell is employed Red-colored bands correspond to dominant $\mathrm{LaNiO}_{3}$ character, while blue-colored bands indicate strong contributions from th interfacial $\mathrm{PbO}$ layer. The tops of the $\mathrm{PbO}$-dominated bands shift from about $-1.2 \mathrm{eV}$ in depletion to approximately $0.5 \mathrm{eV}$ in accumulation. (c) The layer-resolved LDOS at the interface for accumulation and (d) depletion. The $\mathrm{PbTiO}_{3}$ is insulating in depletion and is insulating in accumulation away from the interface, which has LDOS at the Fermi level.

presence of states at the Fermi level in the $\mathrm{PbO}$ layer by examining the band structures shown in Figs. 3(a) (accumulation) and 3(b) (depletion). For the accumulation state [Fig. 3(a)], we observe bands with strong $\mathrm{Pb}$ character at the Fermi level, in which $\mathrm{Pb} 6 s$ states hybridize with $\mathrm{O} 2 p$ states. In the depletion state [Fig. 3(b)], these Pb-related states are absent close to the Fermi level, showing that the bands crossing the Fermi level have no $\mathrm{Pb}$ character and arise exclusively from $\mathrm{Ni}$ and $\mathrm{O}$. Similarly, the layerresolved LDOS is plotted in accumulation [Fig. 3(c)] and depletion [Fig. 3(d)], where it is evident that significant LDOS are present in the interfacial layer of $\mathrm{PbTiO}_{3}$ at the interface in accumulation but not in depletion. The origin of $\mathrm{PbO}$ character in bands that cross the Fermi level is twofold. First, the electric field due to the ferroelectric field effect shifts the $\mathrm{PbO}$ bands upwards to cross the Fermi level in the accumulation state by about $1.7 \mathrm{~V}$, as predicted to occur at vacuum- $\mathrm{PbTiO}_{3}$ surfaces for the same polarization direction [6]. The magnitude of the shift is also consistent with the magnitude of the ferroelectric polarization. Second, the change in the apical-oxygen bon length in the topmost nickelate layer facilitates hybridiza tion between $\mathrm{Ni}$ and $\mathrm{Pb}$. As the apical oxygen move further away from the $\mathrm{NiO}_{2}$ layer in the depletion state hybridization between $\mathrm{Pb}$ states with those of $\mathrm{Ni}$ and $\mathrm{O}$ i suppressed. Density functional theory predicts the appear ance of an additional channel for conduction in the $\mathrm{PbC}$ layer of the ferroelectric. We estimate an upper bound fo the resistivity of this layer by taking the difference in conductance between accumulation and depletior [Fig. 2(c)]. Strikingly, even though the film as a whol has insulating character, the additional conductivity of the channel has a metallic temperature dependence The role of $\mathrm{NiO}_{6}$ and $\mathrm{TiO}_{6}$ octahedral rotations may also be tested by calculating the layer-resolved LDOS using $1 \times 1$ supercell, which suppresses all octahedral tilts an rotations, as shown in Fig. S2. This result indicates that th appearance of a conductive $\mathrm{PbO}$ state at the interface in the accumulation state is not directly related to octahedra rotations. 


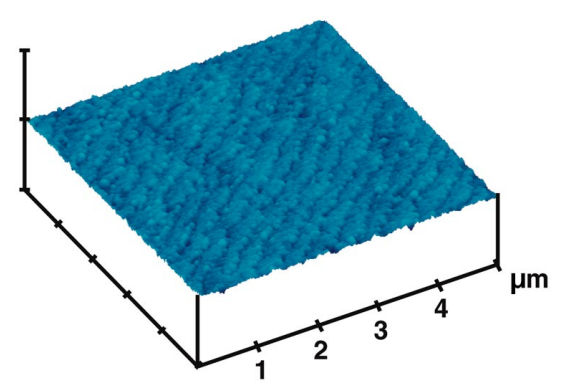

FIG. 1. (Color) Atomic force microscopy image of the surface of a $4 \mathrm{~nm}$ LSMO film. The measured rms surface roughness is 0.1 to $0.2 \mathrm{~nm}$. The image shows a series of terraces spaced a few hundred nanometers apart with a terrace height of $0.2 \mathrm{~nm}$.

Rocking curves taken around the 001 reflection have a typical full width at half maximum of $0.06^{\circ}$. Atomic force microscopy images taken on these films reveal smooth surfaces with atomic steps. Figure 1 shows a $5 \mu \mathrm{m}$ by $5 \mu \mathrm{m}$ scan of a $4 \mathrm{~nm}$ thick LSMO film; the measured root-mean-square (rms) roughness is $0.1-0.2 \mathrm{~nm}^{20}$

Transport measurements taken on a series of ultrathin LSMO films ranging in thickness from $3.7 \mathrm{~nm}$ to $40 \mathrm{~nm}$ are shown in Fig. 2. Films thicker than $4 \mathrm{~nm}$ are insulating at high temperatures and metallic at low temperatures, with a peak in the resistance occurring around $250 \mathrm{~K}$, the ferromagnetic Curie temperature. ${ }^{21}$ For the $4 \mathrm{~nm}$ sample, the peak temperature decreases to around $180 \mathrm{~K}$, while for films thinner than $3.7 \mathrm{~nm}$, the samples are insulating throughout the entire temperature range investigated. This change in transport characteristics is commonly observed in ultrathin LSMO films, where the material becomes insulating, or electrically "dead," below a critical thickness on the order of 3-5 nm, which has been attributed to a modified structure at the interface. ${ }^{22,23}$ Here, $3.8 \mathrm{~nm}$ is the minimum thickness where metallic behavior is observed. Using this information, we grow epitaxial heterostructures of PZT and LSMO, with a PZT thickness of $\sim 300 \mathrm{~nm}$ and an LSMO thickness of $4 \mathrm{~nm}$, just above the dead layer thickness. ${ }^{24}$ The samples are patterned into four-point resistivity paths, and gold electrodes are deposited for transport measurements.

Figure 3(a) shows the temperature dependence of the resistivity of the LSMO layer in a PZT/4 nm LSMO hetero-

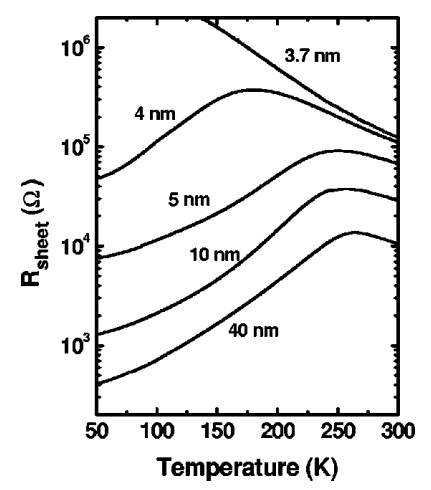

FIG. 2. Sheet resistance as a function of temperature for unpatterned LSMO films of various thicknesses (from top to bottom: $3.7 \mathrm{~nm}, 4 \mathrm{~nm}, 5 \mathrm{~nm}$, $10 \mathrm{~nm}$, and $40 \mathrm{~nm}$ )

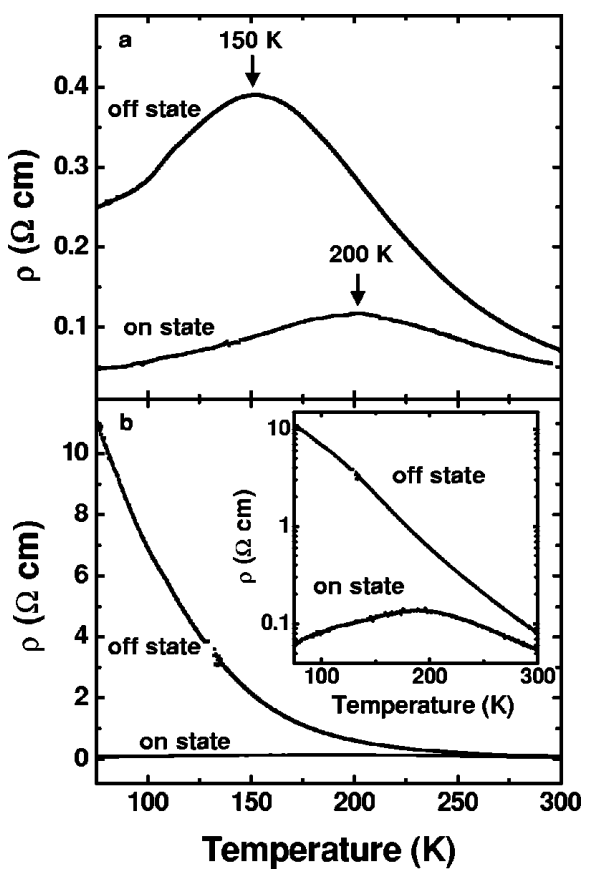

FIG. 3. Resistivity as a function of temperature for: (a) A PZT/4 nm LSMO heterostructure and (b) a PZT/3.8 nm LSMO heterostructure for the two polarization states of PZT. In each graph, the upper curve corresponds to depletion of holes and is termed the off state; the lower curve corresponds to accumulation of holes and is termed the on state. The inset in (b) is a semilog plot of the data.

structure as a function of the polarization state of the PZT layer. For the lower curve, which corresponds to the polarization state that attracts carriers into the LSMO layer (the "on" state), the temperature dependence of the resistivity is similar to what is observed in single layer $4 \mathrm{~nm}$ LSMO films; the film is insulating at high temperatures, followed by metallic behavior below $200 \mathrm{~K}$.

We then apply a $10 \mathrm{~V}$ pulse across the PZT layer to switch the polarization direction, driving carriers out of the LSMO layer and increasing the resistivity (upper curve, the "off" state). As with the on state, the film is insulating at high temperatures, followed by metallic behavior at low temperatures. The temperature at which the transition from insulating to metallic behavior occurs, however, decreases by $50 \mathrm{~K}$. This shift in the transition temperature reflects a decrease in the Curie temperature driven by changes in the carrier concentration. ${ }^{15,25}$ This process is reversible; applying voltage pulses of alternating polarity allows one to switch back and forth between the two resistivity curves.

We then study a heterostructure where the LSMO layer is one atomic layer thinner $(3.8 \mathrm{~nm})$, as shown in Fig. 3(b). For the on state (lower curve), where the polarization adds carriers to the system, the material shows a similar temperature dependence to the $4 \mathrm{~nm}$ LSMO heterostructure, with a slightly lower peak temperature of $190 \mathrm{~K}$ [Fig. 3(b) inset, lower curve]. Applying a $10 \mathrm{~V}$ pulse across the heterostructure reverses the polarization, driving carriers out of the sample, resulting in the upper curve in Fig. 3(b). In depletion, the film now exhibits insulating behavior over the entire temperature range, in sharp contrast with the metallic behavior of the $4 \mathrm{~nm}$ LSMO heterostructure of Fig. 3(a) (in both 

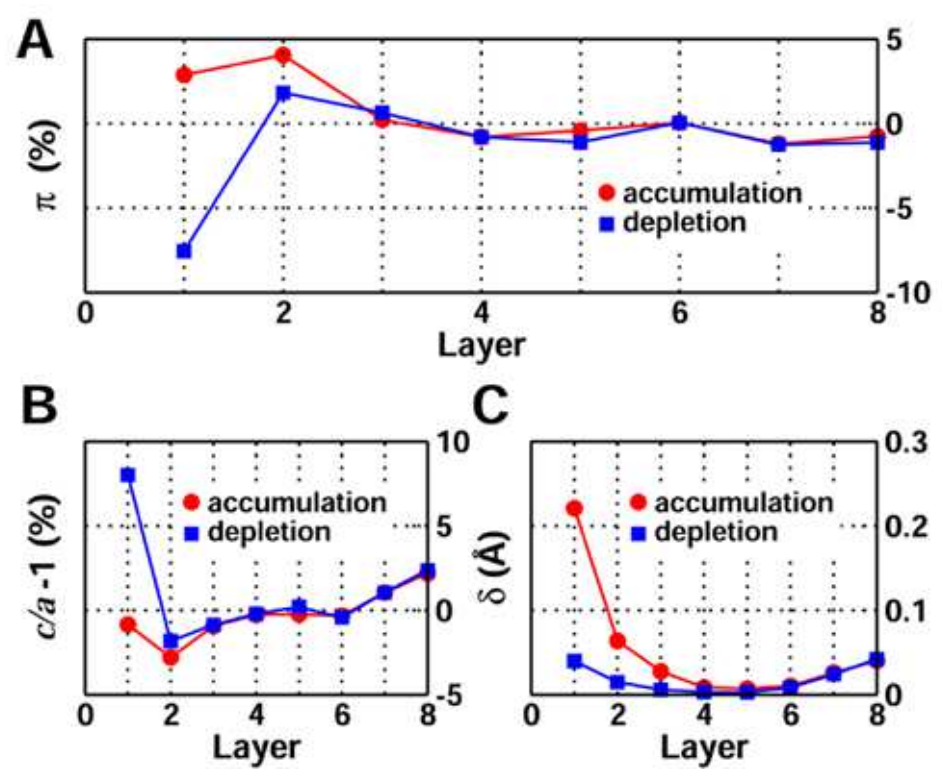

C

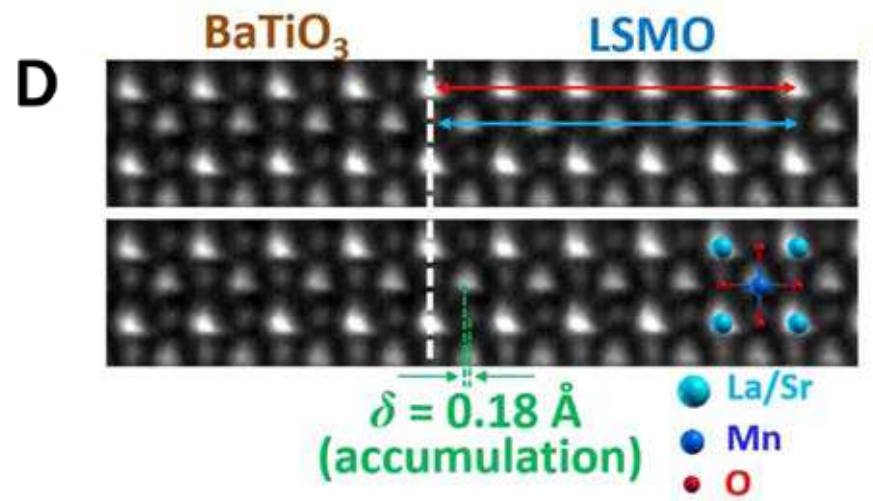



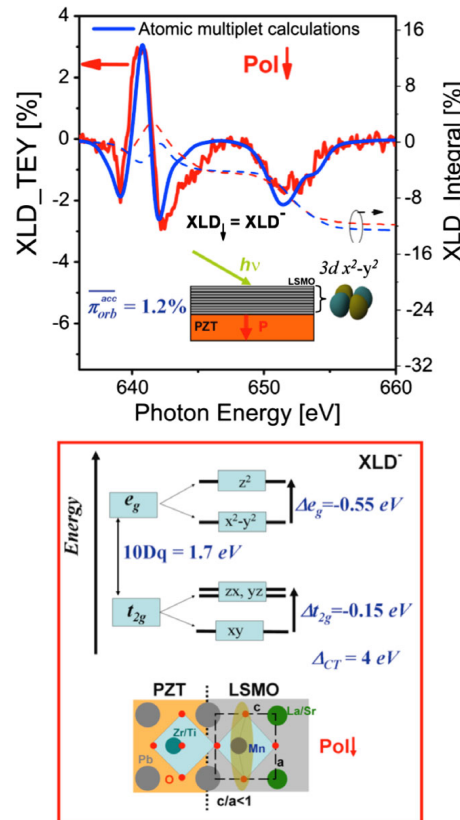

FIG. 3 (color online). Comparison between the atomic multiplet calculations (solid blue lines) and the experimental XLD spectra described in the text for the Pol $\downarrow$ state. The dotted lines represent the integration curves calculated from the XLD spectrum and the related simulation. The inset in the upper panel sketches $\pi_{\text {orb }}$ as a function of the $P$ direction. In the lower panel the energy diagram is related to the $\mathrm{XLD}^{-}$fitting parameters. The schematic ionic model, used to justify the modulation of the orbital occupancy has been re-adapted from Ref. [13]. XAS spectra were measured at $300 \mathrm{~K}$ in order to exclude any possible magnetic contribution.

$$
D_{L}=\frac{\int_{L_{3}+L_{2}}\left(I_{a b}-I_{c}\right) d E}{\int_{L_{3}+L_{2}}\left(2 I_{a b}+I_{c}\right) d E} \simeq \frac{1}{2} \frac{n_{z^{2}}-n_{x^{2}-y^{2}}}{n_{z^{2}}+n_{x^{2}-y^{2}}}=-\frac{1}{2} \pi_{\mathrm{orb}},
$$

where $n_{x^{2}-y^{2}}$ and $n_{z^{2}}$ are the electron occupancy in the $e_{g}$ $3 d_{x^{2}-y^{2}}$ and $3 d_{z^{2}}$ orbitals, respectively. $\pi_{\text {orb }}$ is defined as in Ref. [13]. The integration curves of the experimental XLD spectra for each $P$ state are shown as red and black dotted lines in Figs. 3 and 4, respectively. Notably, $D_{L}$ is negative in the Pol $\downarrow$ state and positive in the Pol $\uparrow$ state. According to Eq. (1) a preferential electron occupancy of the in-plane $3 d_{x^{2}-y^{2}}$ orbitals is favored in the Pol $\downarrow$, while the occupancy of out-of-plane $3 d_{z^{2}}$ ones is favored in the Pol $\uparrow$ state. The data correspond to a change of the average polarization of the $e_{g}$ orbitals from $+1.2 \%$ ( $\mathrm{Pol} \downarrow$ state) to $-0.5 \%$ (Pol $\uparrow$ state), showing that the energy hierarchy of the $\mathrm{Mn}$ $3 d$ states is controlled via $P$ switching. In order to get more information on the changes of the electronic properties of

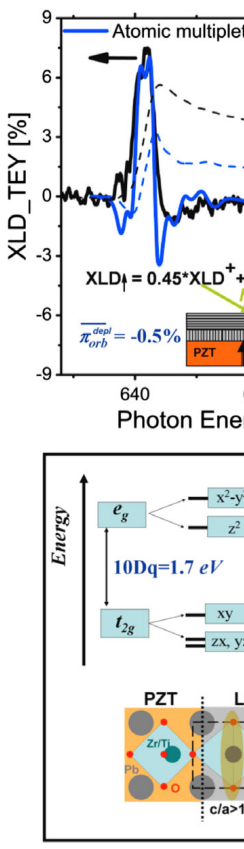

FIG. 4 (color online). Same as Pol $\uparrow$ state.

the LSMO layer as a function o splitting calculations to simulc [27]. Because of the low level LSMO layer, i.e., $x=0.175$, w ration for the Mn ions in $D_{4 h}$ syl of $1.7 \mathrm{eV}$, and a nonzero splittin plane $\mathrm{Mn} 3 d t_{2 g}$ and $e_{g}$ orbitals $\Delta e_{g}=E_{x^{2}-y^{2}}-E_{z^{2}}$. The partial $\mathrm{Mn} 3 d-\mathrm{O} 2 p$ bonding was by charge transfer (CT) effects transfer energy $\Delta_{\mathrm{CT}}$ and by Coulomb energy associated to $t$ and the $U_{d d}$ Hubbard potenti [28]. The XLD spectrum obtain is reasonably reproduced by electronic state for the whole L solid blue line of Fig. 3 obtai $\Delta t_{2 g}=-0.15, \Delta e_{g}=-0.55$, a parameters that simulates at $\mathrm{b}$ designed as $\mathrm{XLD}^{-}$, while the co is shown in the bottom panel of $\mathrm{I}$ XLD spectrum is also obtained layer outside the channel, i.e., switched (no gate electrode). T as-grown $P$ state of the FEFED from the upper LSMO layer). 

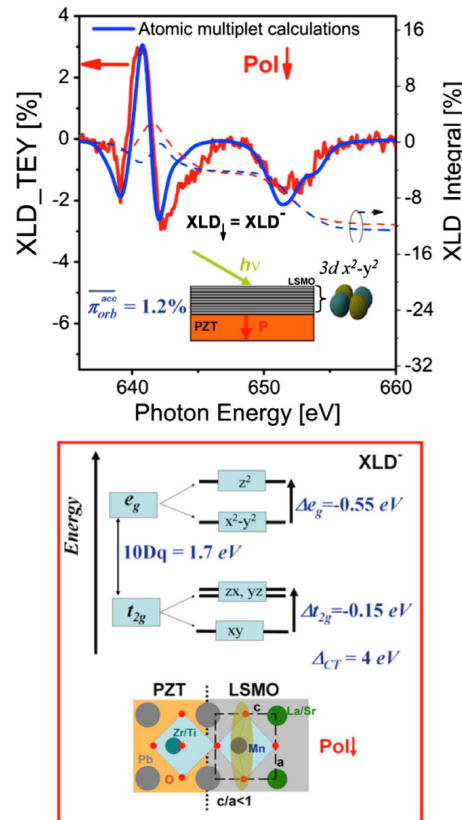

FIG. 3 (color online). Comparison between the atomic multiplet calculations (solid blue lines) and the experimental XLD spectra described in the text for the Pol $\downarrow$ state. The dotted lines represent the integration curves calculated from the XLD spectrum and the related simulation. The inset in the upper panel sketches $\pi_{\mathrm{orb}}$ as a function of the $P$ direction. In the lower panel the energy diagram is related to the $\mathrm{XLD}^{-}$fitting parameters. The schematic ionic model, used to justify the modulation of the orbital occupancy has been re-adapted from Ref. [13]. XAS spectra were measured at $300 \mathrm{~K}$ in order to exclude any possible magnetic contribution

$$
D_{L}=\frac{\int_{L_{3}+L_{2}}\left(I_{a b}-I_{c}\right) d E}{\int_{L_{3}+L_{2}}\left(2 I_{a b}+I_{c}\right) d E} \simeq \frac{1}{2} \frac{n_{z^{2}}-n_{x^{2}-y^{2}}}{n_{z^{2}}+n_{x^{2}-y^{2}}}=-\frac{1}{2} \pi_{\mathrm{orb}},
$$

where $n_{x^{2}-y^{2}}$ and $n_{z^{2}}$ are the electron occupancy in the $e_{g}$ $3 d_{x^{2}-y^{2}}$ and $3 d_{z^{2}}$ orbitals, respectively. $\pi_{\text {orb }}$ is defined as in Ref. [13]. The integration curves of the experimental XLD spectra for each $P$ state are shown as red and black dotted lines in Figs. 3 and 4, respectively. Notably, $D_{L}$ is negative in the Pol $\downarrow$ state and positive in the Pol $\uparrow$ state. According to Eq. (1) a preferential electron occupancy of the in-plane $3 d_{x^{2}-y^{2}}$ orbitals is favored in the Pol $\downarrow$, while the occupancy of out-of-plane $3 d_{z^{2}}$ ones is favored in the Pol $\uparrow$ state. The data correspond to a change of the average polarization of the $e_{g}$ orbitals from $+1.2 \%$ ( $\mathrm{Pol} \downarrow$ state) to $-0.5 \%$ (Pol $\uparrow$ state), showing that the energy hierarchy of the Mn $3 d$ states is controlled via $P$ switching. In order to get more information on the changes of the electronic properties of

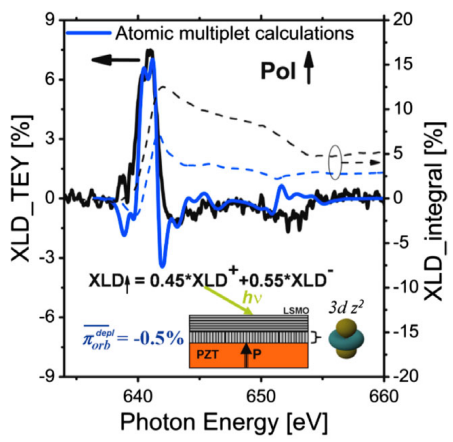

9 OCTOBER 2015

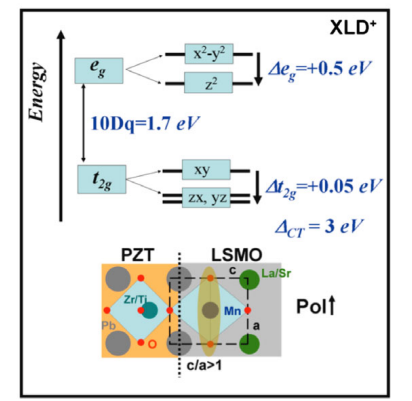

FIG. 4 (color online). Same as in Fig. 3 but related to the Pol $\uparrow$ state.

the LSMO layer as a function of $P$, we performed multiplet splitting calculations to simulate the observed XLD data [27]. Because of the low level of chemical doping of the LSMO layer, i.e., $x=0.175$, we assumed a $\mathrm{Mn}^{3+}$ configuration for the Mn ions in $D_{4 h}$ symmetry, a crystal field $10 D q$ of $1.7 \mathrm{eV}$, and a nonzero splitting of the in-plane and out-ofplane Mn $3 d t_{2 g}$ and $e_{g}$ orbitals, i.e., $\Delta t_{2 g}=E_{x y}-E_{x z, y z}$ and $\Delta e_{g}=E_{x^{2}-y^{2}}-E_{z^{2}}$. The partially covalent nature of the $\mathrm{Mn} 3 d-\mathrm{O} 2 p$ bonding was modeled in the calculation by charge transfer (CT) effects parametrized by the charge transfer energy $\Delta_{\mathrm{CT}}$ and by the difference between the Coulomb energy associated to the core-hole interaction $U_{p d}$ and the $U_{d d}$ Hubbard potential, i.e., $U_{p d}-U_{d d}=1.5 \mathrm{eV}$ [28]. The XLD spectrum obtained in the Pol $\downarrow$ state $\left(\mathrm{XLD}_{\downarrow}\right)$ is reasonably reproduced by assuming an homogeneous electronic state for the whole LSMO film, as shown by the solid blue line of Fig. 3 obtained with fitting parameters $\Delta t_{2 g}=-0.15, \Delta e_{g}=-0.55$, and $\Delta_{\mathrm{CT}}$ of $4 \mathrm{eV}$. The set of parameters that simulates at best the $\mathrm{XLD}_{\downarrow}$ spectrum is designed as $\mathrm{XLD}^{-}$, while the corresponding energy diagram is shown in the bottom panel of Fig. 3. Interestingly, a similar XLD spectrum is also obtained in areas of the upper LSMO layer outside the channel, i.e., the pads, which cannot be switched (no gate electrode). This result confirms that the as-grown $P$ state of the FEFEDs is Pol $\downarrow$ ( $P$ pointing away from the upper LSMO layer). This $P$ direction determines 


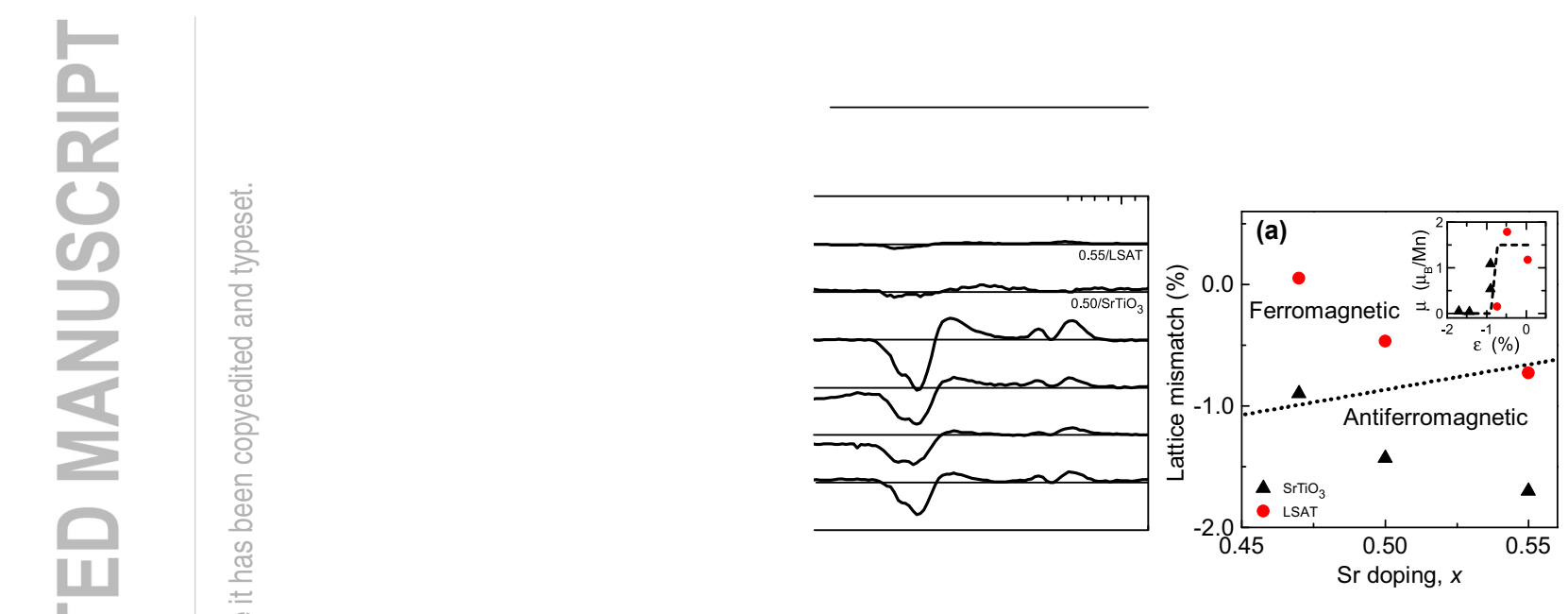


(one magnetic region) and nonsuppressed surface magnetization (two magnetic regions) clearly indicate that including suppressed surface magnetization provides the best fit (Fig. S3). Given that the film is atomically flat (roughness of $\sim 4 \AA$ ) and has an XRD rocking curve full-width-at-halfmaximum value of less than $0.05^{\circ}$, the suppressed magnetization at the surface is likely due to factors other than structural deterioration, e.g., preferential formation of oxygen vacancies often reported for perovskite oxides such as STO [34-36].

In order to test whether this surface region is sensitive to electrostatic doping, we deposited an $\sim 8.4 \mathrm{~nm}$ layer of FE PZT on top of LSMO grown under identical conditions as the first sample. Using piezoresponse force microscopy, we observed that the polarization of PZT naturally points (self-poles) towards the PZT/LSMO interface. Polarization pointing towards the interface will induce hole depletion at the interface when the FM is a metal (as is the case for our LSMO film) (Fig. S4). In Figs. 2(d)-2(f), the PNR data for the PZT/LSMO sample are shown. In Fig. 2(f), the $n$ SLD is similar to the uncapped LSMO film, but the magnetic profile is different. Instead of suppressed magnetization at the LSMO surface, remarkably the magnetization is enhanced to $617 \mathrm{kA} / \mathrm{m}\left(4.0 \mu_{\mathrm{B}} /\right.$ f.u. $)$, which is larger than the magnetization for the first sample (the LSMO film without PZT).

To illustrate the confidence that we have with our fitting parameters, we have provided alternative simulations in Fig. S5, which do not allow for enhanced magnetization. It is clear that neither suppressed surface magnetization nor uniform magnetization accurately explain our experimental data. In order to further support the confidence in our PNR fitting parameters, we note $M_{\text {sat }}$ obtained from magnetization versus field loops from SQUID and the integrated magnetization values determined from the different magnetic layers found by PNR in Fig. S5 show excellent agreement.

The thickness of the enhanced magnetic region (region III) of $2.0( \pm 1) \mathrm{nm}$ we inferred from the PNR experiment is similar to the length scale of $\sim 3-5$ unit cells reported for charge screening in metallic LSMO [4,12,21]. Interestingly, the magnetization within the bulk region of the bilayer film (region II) increased from $451 \mathrm{kA} / \mathrm{m}\left(3.0 \mu_{\mathrm{B}} /\right.$ f.u.) to $540 \mathrm{kA} / \mathrm{m}$ ( $3.6 \mu_{\mathrm{B}} /$ f.u.) for the uncapped manganite film. The use of the term "bulk" for region II is used to specify the area of the film that is most unaffected by the substrate, a capping layer. or air. The notable increase in magnetization for the film bulk suggests that there could be an effect of capping in addition to the interfacial magnetization enhancement from the presence of the FE. It is worth noting that capping of ultrathin films has also been shown to impact the conductivity with respect to a single layer film [37]. Capping of films can influence strain, however, reciprocal space mapping of our samples indicates that both samples are coherently strained to the substrate lattice (Fig. S6). Therefore, since the strain of these samples appears to be the same, strain does not seem a likely origin for changes of the magnetism we observed.

It is intuitive to assume that the enhanced magnetization directly at the PZT/LSMO interface is a consequence of the FE polarization, which is believed to occur on a short length scale of a few unit cells $[4,38,39]$. In fact, our PZT has one of the largest reported polarization values of $80 \mu \mathrm{C} / \mathrm{cm}^{2}$, capable of inducing a change in carriers up to $0.8 e^{-} /$unit cell [40].

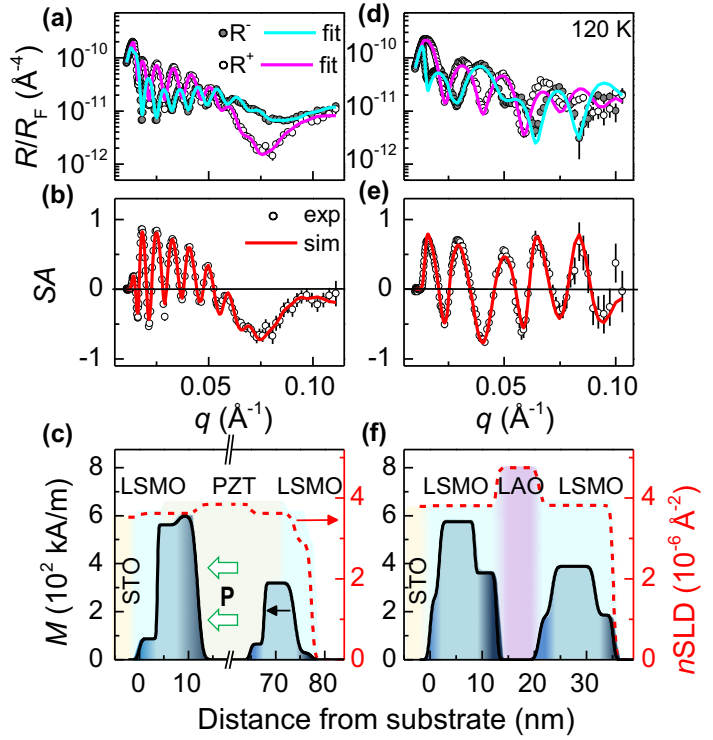

FIG. 3. Normalized neutron reflectivity, spin asymmetry, magnetic profile, and $n$ SLD are shown in (a)-(c) for LSMO/PZT/LSMO and (d)-(f) for LSMO/LAO/LSMO layers on STO. A scale break has been added in (c) for clarity of the LSMO regions. Suppressed magnetization at the STO/LSMO interface is shown for both samples, whereas the LSMO/PZT/LSMO sample shows enhanced and diminished magnetization. Comparison with the LSMO/LAO/LSMO sample, which shows lower magnetization at the LSMO/LAO interface, confirms the field effect as the primary role for enhanced magnetization in LSMO in PZT/LSMO heterostructures.

Assuming that the magnetic interface thickness of $2 \mathrm{~nm}$ is proxy for the Thomas-Fermi screening length, then the PZT layer can induce a charge density in the LSMO interface of $\pm 2.5 \times 10^{21} \mathrm{~cm}^{-3}$. This electrostatic doping equates to a compositional change in Sr content as high as $\Delta x= \pm 0.15$. In order to test the hypothesis that the interface magnetization is affected by electrostatic doping, we have grown a trilayer structure of two LSMO layers with a $50 \mathrm{~nm}$ thick PZT interlayer. This sample provides the opportunity to observe the effects of both hole accumulation and depletion at opposite interfaces. Analysis of the PNR data shown Figs. 3(a)-3(c) indicates that the magnetization of $\mathrm{FM} / \mathrm{FE}$ interface (i.e., the interface formed by putting PZT on top of LSMO) is enhanced with respect to the uncapped LSMO sample (the first sample) and consistent with the enhancement observed for the second sample-the FE capped sample. Furthermore, the magnetization of the FE/FM interface (i.e., the interface formed by putting LSMO on top of PZT) is suppressed more than observed for the uncapped LSMO interface or surface. These observations are consistent with self-poling of the PZT film remaining the same as observed for the second sample, i.e., polarization pointing towards the FM/FE (bottom-most) interface. These observations agree with our expectation that hole accumulation/depletion is restricted to within a few nanometers of the interface. Due to the thickness of the PZT 

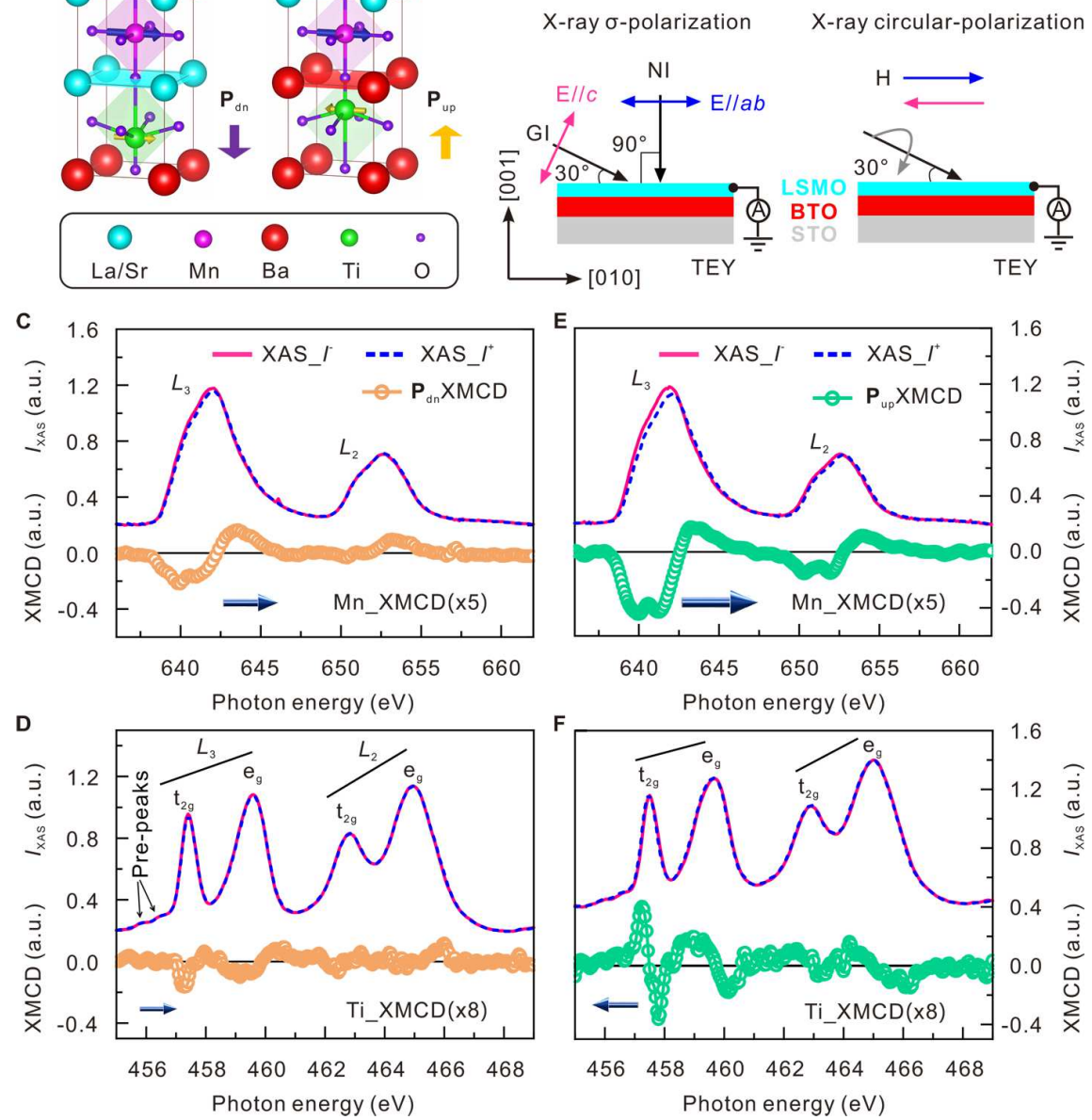

Figure 2. Normalized X-ray absorption spectroscopy and X-ray magnetic circular dichroism of the LSMO/BTO heterostru Schematic illustrations of the LSMO/BTO heterointerfaces and arrangement of magnetic moment dependent on FE polarizati XLD and XMCD techniques, where GI and NI represent grazing incidence (polarization $E / / c$ ) and normal incidence X-ray ( respectively. (C) and (D) Mn- and Ti- $L_{2,3}$ edges XAS under $\pm 1 \mathrm{~T}$ in-plane magnetic field and their XMCD of LSMO/BTC polarization. (E) and (F) Mn- and Ti- $L_{2,3}$ edges XAS under $\pm 1 \mathrm{~T}$ in-plane magnetic field and their XMCD of LSMO/BT polarization. The arrows in the inset show the direction of the total magnetic moment at $\mathrm{Ti}$ and $\mathrm{Mn}$.

terrace separated by $\sim 0.40 \pm 0.05 \mathrm{~nm}$ high step reveals a twodimensional growth of films, which is further supported by in situ RHEED of various oxide materials (Figure S1). We obtained the SrO-terminated surface through depositing a 1 u.c.-SrRuO 3 (SRO) layer on $\mathrm{TiO}_{2}$-terminated STO substrates at high temperature and low oxygen pressure (Figure S1), benefiting from that, a highly volatile $\mathrm{RuO}_{2}$-layer will be desorbed before the deposition of BTO film. ${ }^{23}$ The out-ofplane piezoresponse force microscopy (PFM) images of BTO written by an electrical bias of $\mp 6 \mathrm{~V}$ at room temperature are shown in Figure 1C,D. A clear phase contrast of $\sim 180^{\circ}$ shows high quality and switchability of FE polarization in BTO films, which is further supported by the butterfly-like amplitude and hysteresis phase loop (Figure S2A,B). More importantly, we deduce that the FE polarization of BTO terminated by $\mathrm{SrO}$, which is switched by a positive electrical bias through Coulomb interaction between the conductive tip and bound charges, is upward $\left(\mathbf{P}_{\text {up }}\right)$, whereas the FE polarization of BTO terminated by $\mathrm{TiO}_{2}$ which is switched by a negative electrical bias is downward $\left(\mathbf{P}_{\mathrm{dn}}\right)$, as shown in Figure S3. Thus, we could conclude that the preferential FE polarization in the as-grown
BTO terminated by $\mathrm{TiO}_{2}(\mathrm{SrO})$ has a $\mathbf{P}_{\mathrm{dn}}(\mathbf{I}$ also confirm that the 5 u.c. LSMO does original orientation of the FE polarization in Figure S2B-E. Concerning the effect of ter ferroelectricity of fully stained films, the rem (without electrical bias) ${ }^{27}$ has an increa decreasing temperature, revealing no struc sition. On the other hand, theoretical calcula ultrathin BTO films keep the $c$-axis tetragor FE polarization does not rotate at low tem compressive strain of $-2.0 \%$ imposed by substrate. ${ }^{28}$ Hence, it is reasonable that BT single FE domain $\left(\mathbf{P}_{z}>\mathbf{P}_{x}=\mathbf{P}_{y}=0\right)$ from $\mathrm{r}$ to $80 \mathrm{~K}$. Reciprocal space mappings (RSMs) $1 \mathrm{E}$ and Figure $\mathrm{S} 4$ suggest that both BTO fil grown on $\mathrm{TiO}_{2}$ - and SrO-terminated STO s grown films have a tetragonal structure unde strain of $-2.22 \%$ (Table S1) and possess strained state (lattice parameters: $a=b=3.9$ and tetragonality $=1.11$ ), as indicated by the comparison with the lattice parameters of $\mathrm{B}^{\text {? }}$ 

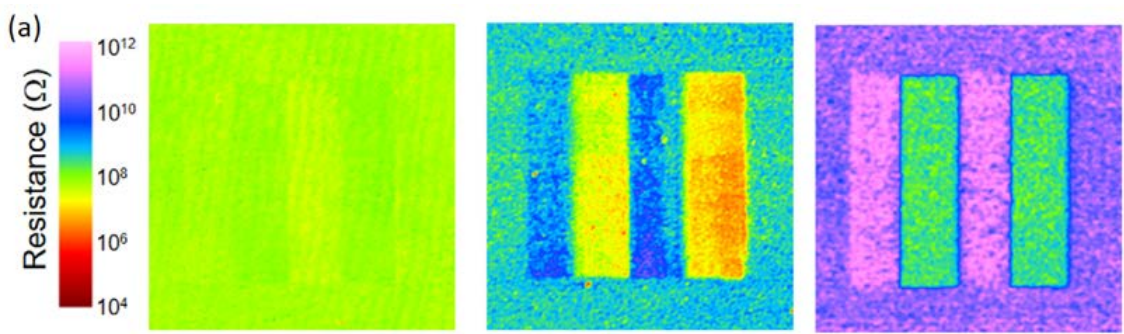

(b) Bad metal Ferroelectric Good metal
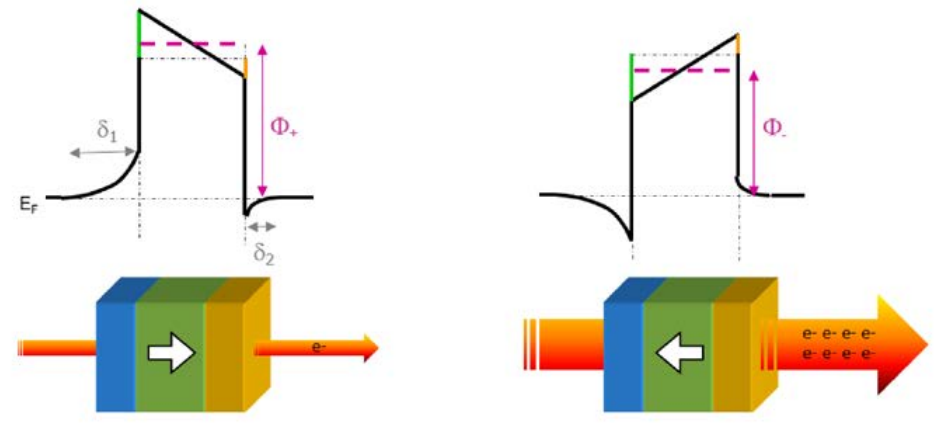

(1)

a

U

U $\stackrel{\oplus}{\leftrightarrows}$

(1)

1 오 썼

(C)

(0)

(1)

(2)

4

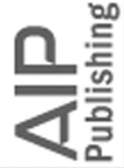



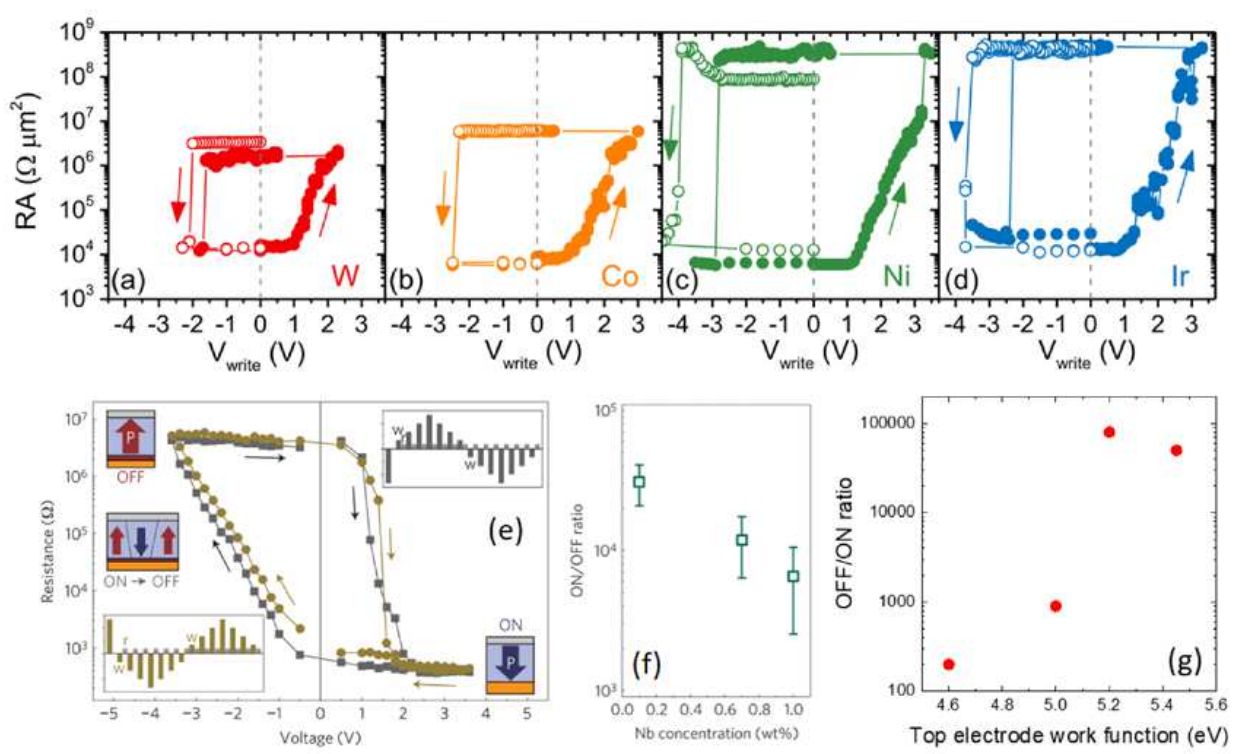

$\frac{0}{3}$ 

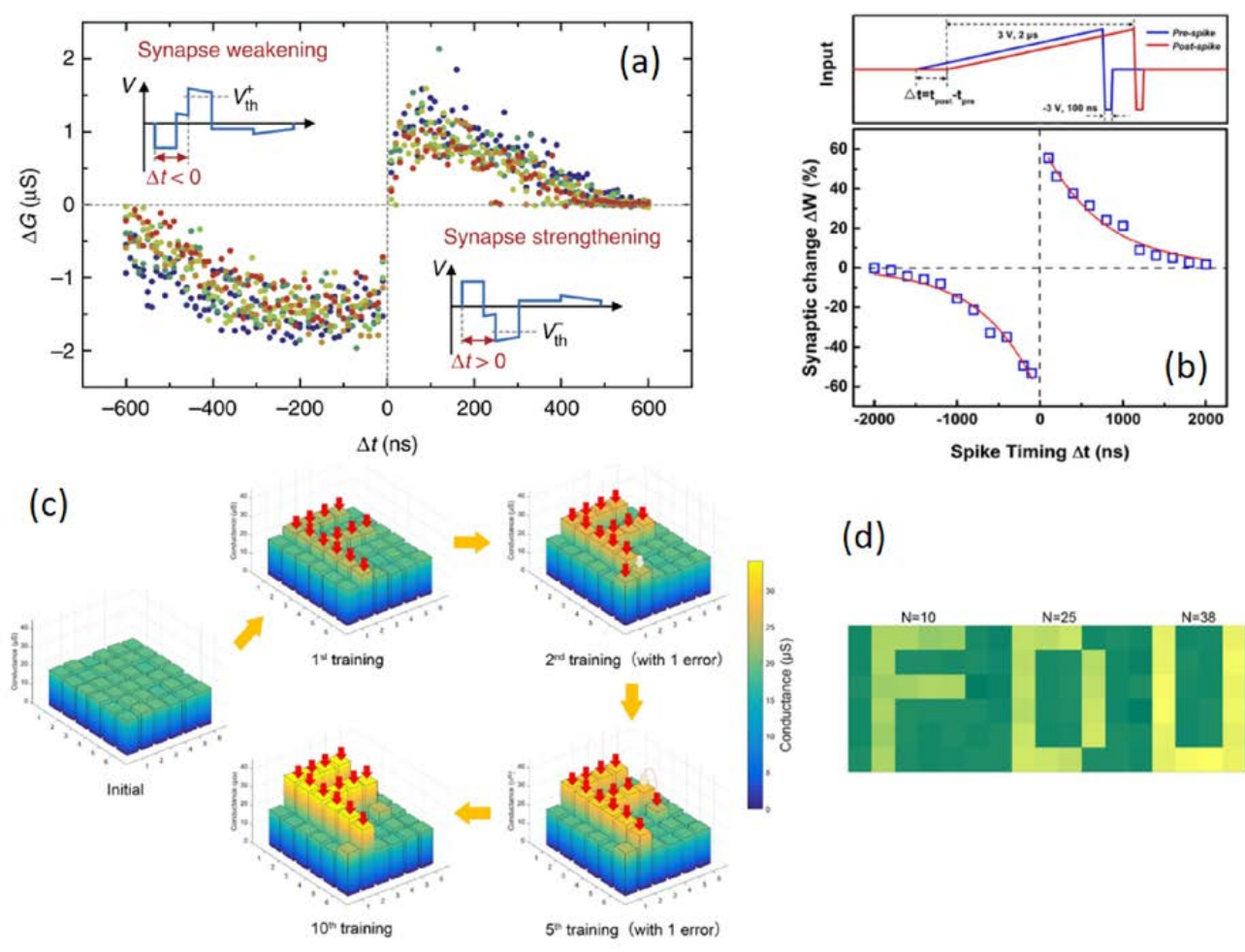

(d)

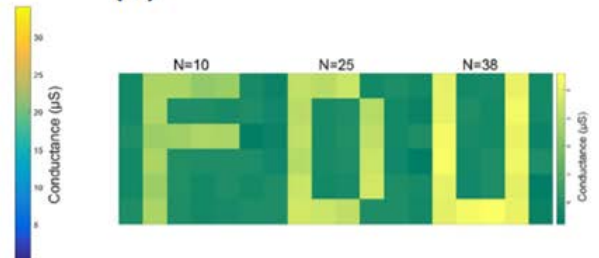




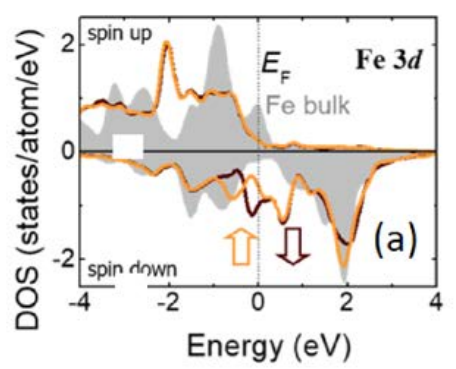

(b)

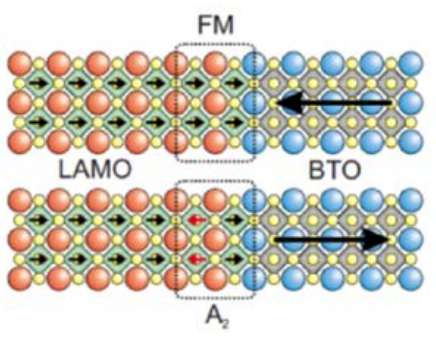



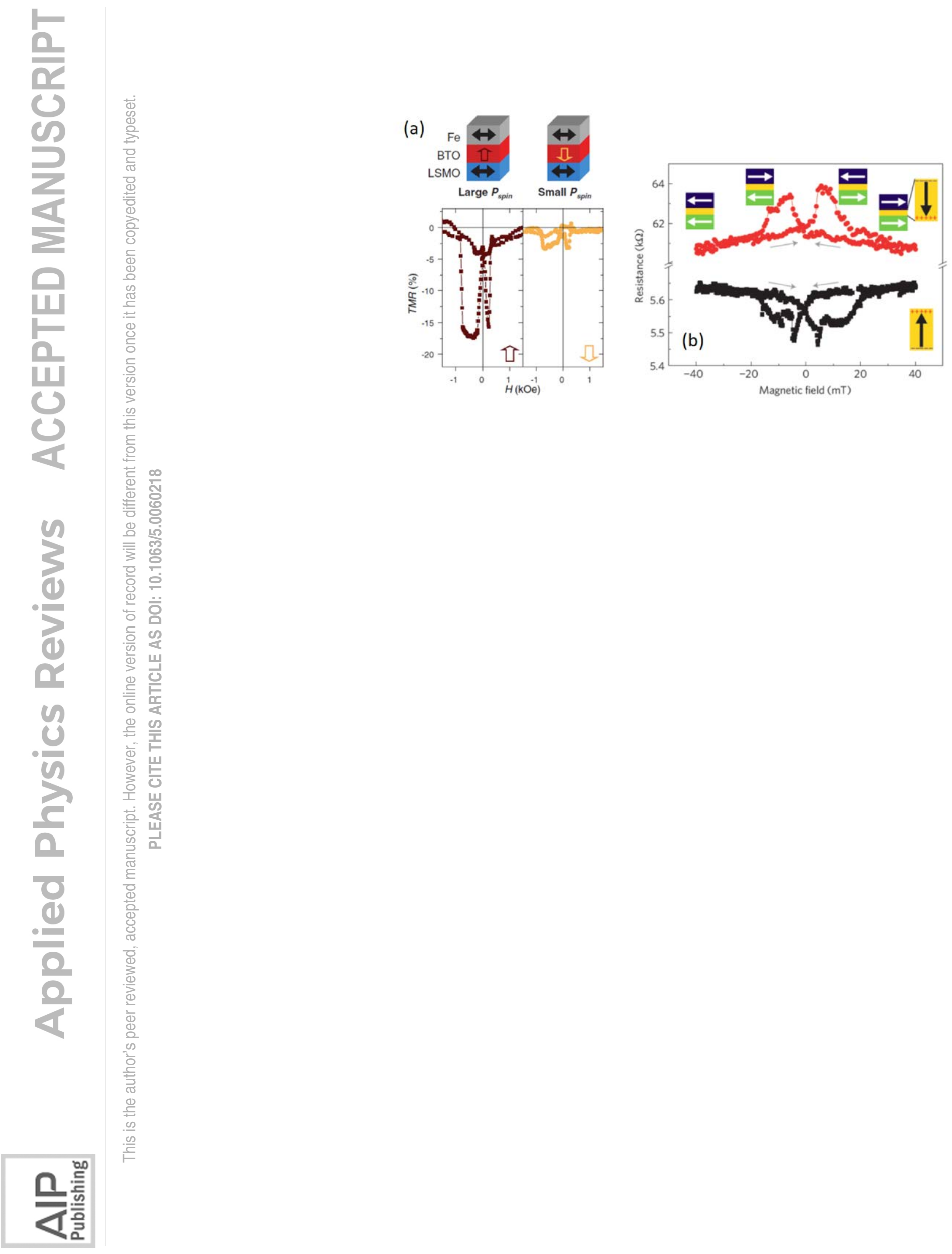

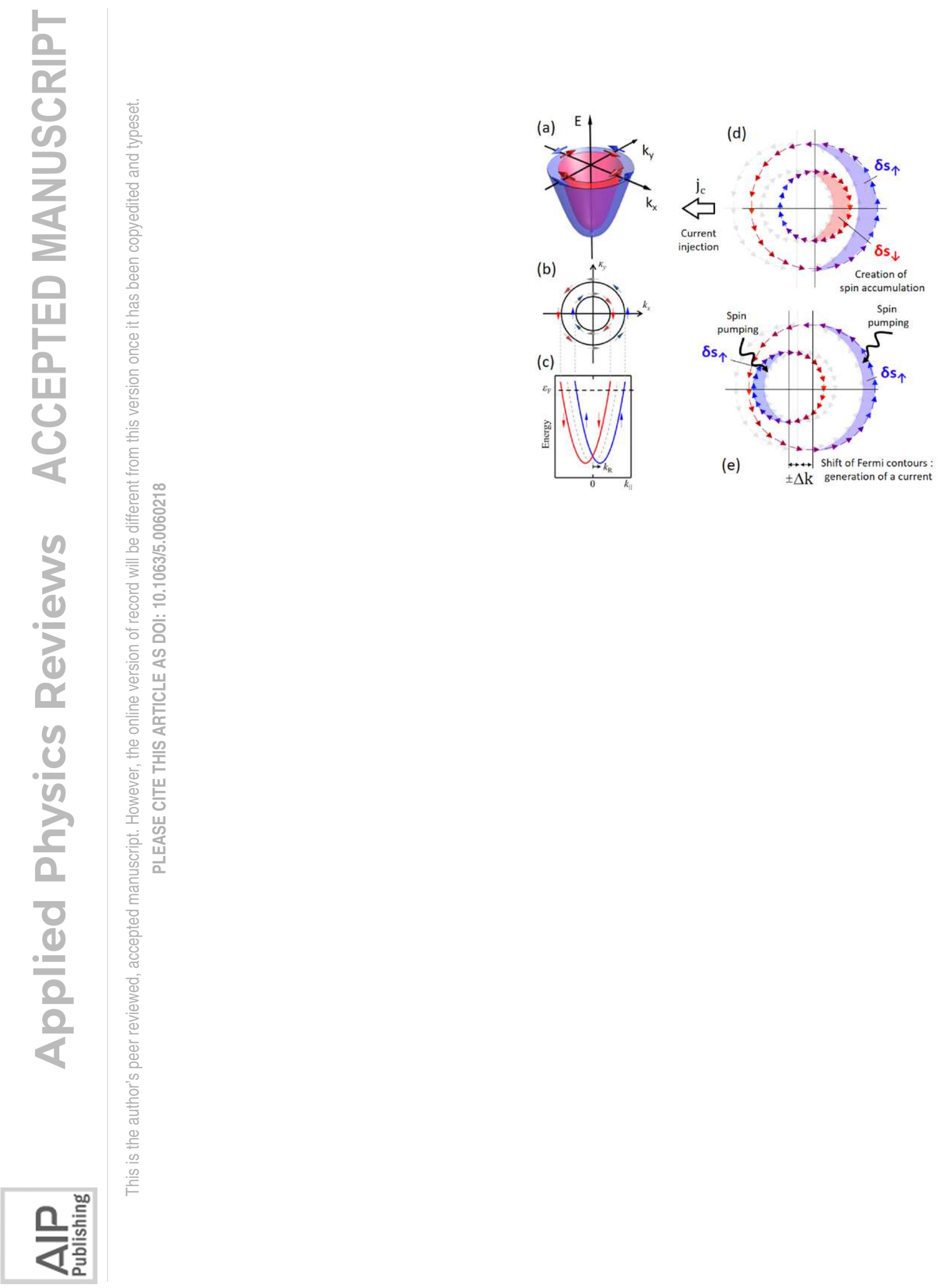
Article

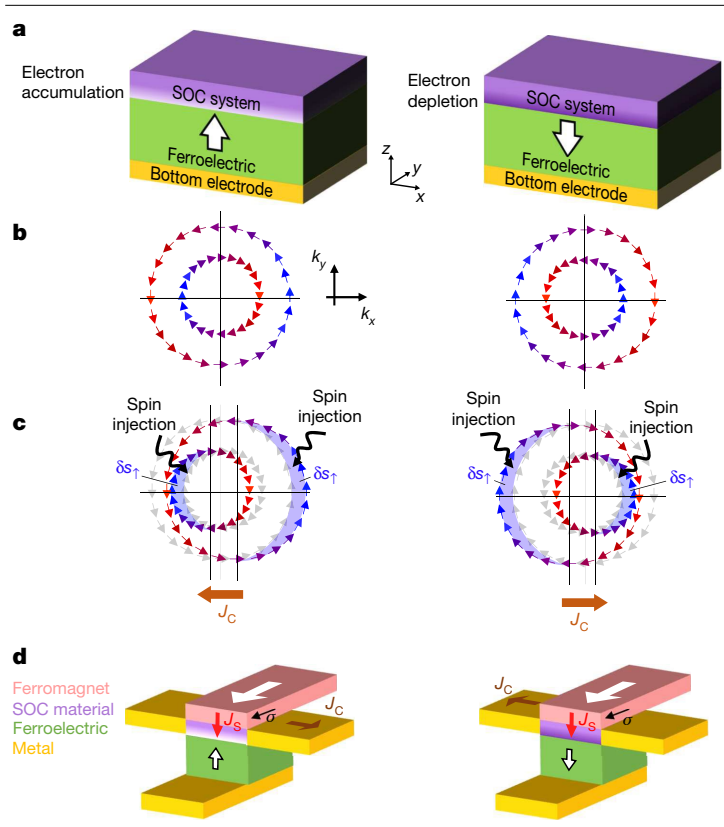

Fig. 1 | Concept of ferroelectricity-controlled spin-charge conversion. a, Sketch of a ferroelectric Rashba architecture combining a ferroelectric material (green) and a material with spin-orbit coupling (SOC; purple). Upon switching polarization, electrons are accumulated (left) or depleted (right) in the SOC material (for example, a 2DEG), creating an electric field whose sign depends on the polarization direction. b, Corresponding Rashba-split chiral Fermi contours with spin-momentum locking. The chirality of the contours switches upon switching the ferroelectric polarization. $k_{x}$ and $k_{y}$ correspond to the $x$ and $y$ axes in momentum space. Blue and red colours indicate the spin direction (blue, along $k_{y}$; red, along $-k_{y}$ ). c, Inverse Edelstein effect in a Rashba interface. When a spin current is injected (for example, by spin pumping) with a spin polarization along the $y$ axis, the spin population is altered, causing a displacement of the two inequivalent Fermi surfaces (red and blue lines) by $\pm \Delta k$ in momentum space. This results in a net charge current generated perpendicularly to the spin current and to its spin polarization. The sign of the generated current depends on the chirality of the Fermi contours and is thus reversed upon switching ferroelectric polarization. $\delta s_{\uparrow}$ corresponds to the injected excess of spin-up density. d, Non-volatile device operated by ferroelectricity and Rashba SOC. Through the inverse Edelstein effect a charge current $J_{\mathrm{C}}$ is generated by the conversion of a spin current $J_{\mathrm{s}}$ injected from the ferromagnet. The sign of $J_{c}$ changes with the direction of the ferroelectric polarization. The large white arrows show the ferromagnet magnetization, the small black arrows the spin $\sigma$, the brown arrows the direction of the charge current $J_{\mathrm{c}}$, and the red arrow the direction of the spin current $J_{\mathrm{s}}$. The small black-and-white arrows correspond to the ferroelectric polarization.

Fermi contours (Fig. 1b). Through the inverse Edelstein effect ${ }^{13}$, the injection of a spin current into the Rashba state will produce a charge current $J_{\mathrm{C}}$ whose sign will depend on the ferroelectric polarization state (Fig. 1c). This mechanism offers the possibility to design a wealth of devices such as the bipolar memory proposed in Fig. 1. It can also be the basis of logic devices ${ }^{22}$ akin to the magnetoelectric spin-orbit (MESO) device proposed by Intel ${ }^{23}$, but without resorting to a multiferroic to switch the ferromagnet.

To experimentally demonstrate the non-volatile electric control of the spin-charge conversion, we use $\mathrm{SrTiO}_{3}$ (STO) 2DEGs, generated by the deposition of a film of Al onto a STO single crystal ${ }^{24,25}$. Indeed, STO 2DEGs exhibit a sizeable Rashba $S \mathrm{CC}^{10}$ with a very high conversion efficiency ${ }^{25,26}$. In addition, STO is a quantum paraelectric that develops an electric-field-induced switch perature ${ }^{7-9}$.

The spin-to-charge conversion was me ing by ferromagnetic resonance on a $\mathrm{Ni}$ sample (see Fig. 2a inset). The nominally 5 was thinned down to $250 \pm 20 \mu \mathrm{m}$ using $\mathrm{n}$ ing the application of high electric fields was applied along the $y$ direction. At the pure spin current is injected into the 2DEC spins oriented along $y\left(\right.$ ref. $\left.^{27}\right)$. The meas ing due to this relaxation channel allows spin current $^{26,27}$. In the $2 \mathrm{DEG}$, this spin cur charge current oriented along $x$ by the in the sample is in open circuit, at the reso voltage drop along the sample, in the $x \mathrm{di}$

In the pristine, ungated state, the voltage corresponds to the production of a posi $1.2 \mathrm{~A} \mathrm{mT}^{-2} \mathrm{~m}^{-1}$ (top left panel of Fig. $2 \mathrm{~b}$ ). known to undergo a phase transition at large electric field has been applied, the ma remanent polarization. This phenomen field-induced ferroelectric order or a fiel state. We applied voltages up to $\pm 200 \mathrm{~V}$ $\pm 8 \mathrm{kV} \mathrm{cm}^{-1}$, high enough to achieve this $\mathrm{ph}$ initialization cycle $[+200 \mathrm{~V} ;-200 \mathrm{~V} ;+200$ ence of the spin-pumping signal shows a $h$ The charge currents produced at ferromas site signs for $+200 \mathrm{~V}$ and $-200 \mathrm{~V}$ gate vo $F$ and D of Fig. 2a and b. After applying the malized current reaches a very high amplits the record values obtained previously in $\mathrm{L}$ ples (around $\left.5 \mathrm{~A} \mathrm{mT}^{-2} \mathrm{~m}^{-1}\right)^{26}$. The spin-cha quantified by the inverse Edelstein length $2 \mathrm{D}$ charge current density produced by $\mathrm{t}$ that is, $\lambda_{\mathrm{IEE}}=J_{\mathrm{C}}^{2 \mathrm{D}} / J_{\mathrm{S}}^{3 \mathrm{D}}$ (ref. ${ }^{17}$; see Methods). $\mathrm{H}$ a value one to two orders of magnitude la interfaces ${ }^{17}$ or topological insulators ${ }^{28}$.

We note that the produced current-an version rate-is remanent at gate voltage in Fig. 2a and b. Similar hysteretic behavi several thinned-down samples but not 0 strate, which indicates the existence of a hysteresis to appear. The non-volatile con version is further evidenced by Fig. 2c, wh charge current produced at $0 \mathrm{~V}$ after the a of $\pm 200 \mathrm{~V}$ gate voltage. Figure $2 \mathrm{~d}$ shows th of the difference $\Delta I_{\mathrm{C}}$ in the produced cur after applying pulses of $+200 \mathrm{~V}$ and $-200 \mathrm{~V}$ and vanishes above $45-50 \mathrm{~K}$, suggesting paraelectric phase ${ }^{7-9}$. Extended Data Figs. is reproducible and stable in time for at le

We have also performed electric polar $\mathrm{Al}(1.8 \mathrm{~nm}) / / \mathrm{STO} 2 \mathrm{DEG}$ sample with a STO visible in Fig. 3a, the application of an el (green curve) yields a linear dependence expected for a dielectric. However, when $7 \mathrm{kV} \mathrm{cm}^{-1}$, a hysteresis develops, associa peaks in the /versus $E$ data (Fig. 3a inset). is about $4 \mu \mathrm{Ccm}^{-2}$, in agreement with ear ing the temperature (Fig. 3c), the loop pr ing a Curie temperature close to $50 \mathrm{~K}$ (Fig with the temperature at which the reman effect vanishes (Fig. 2d), strongly sugg polarization is at the origin of the hystere At low temperature, reducing $E$ to below 


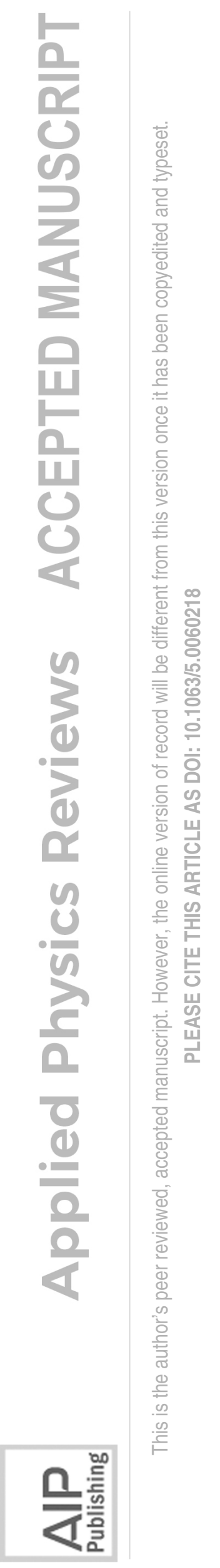

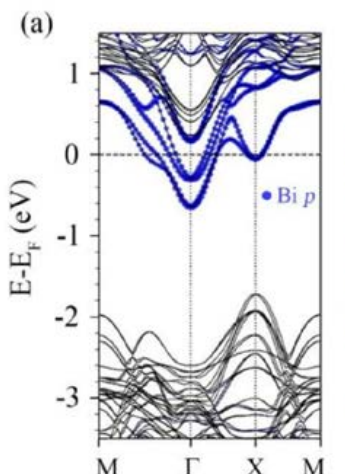

(c)

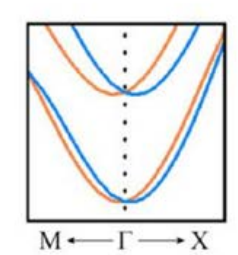

(b)
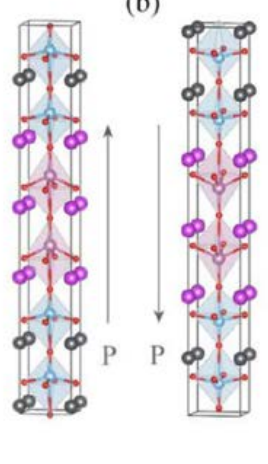

(d)

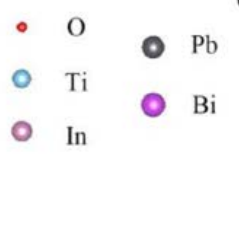

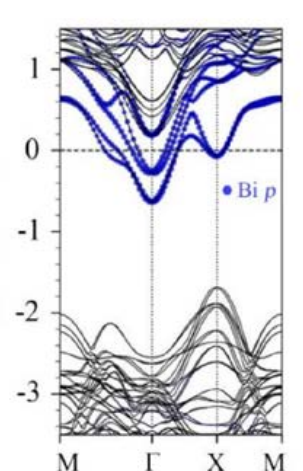

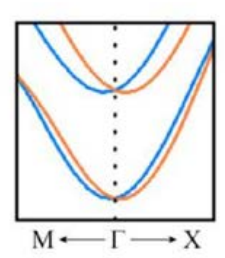

